Dr. 2106

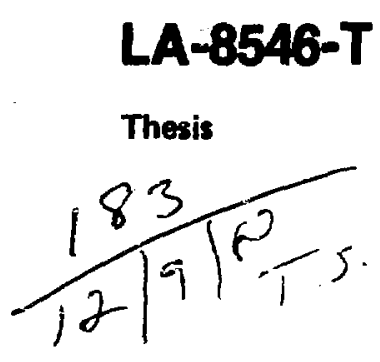

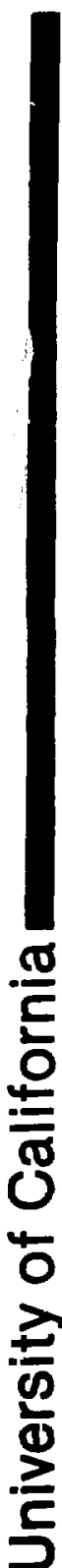
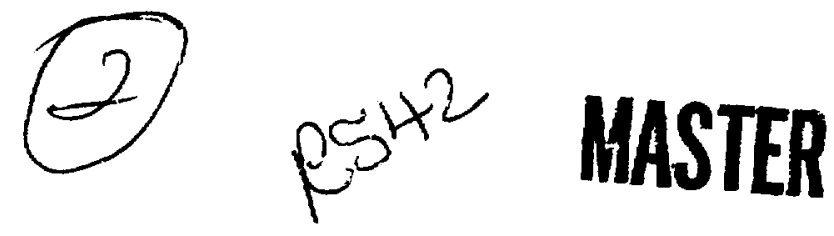

Minimizing the Cost of Splitting in Monte Carlo Radiation Transport Simulation 
LA-8546-T

Thesis

UC-32

Issued: October 1980

\title{
Minimizing the Cost of Splitting in \\ Monte Carlo Radiation Transport Simulation
}

\author{
Raymond John Juzaitis
}
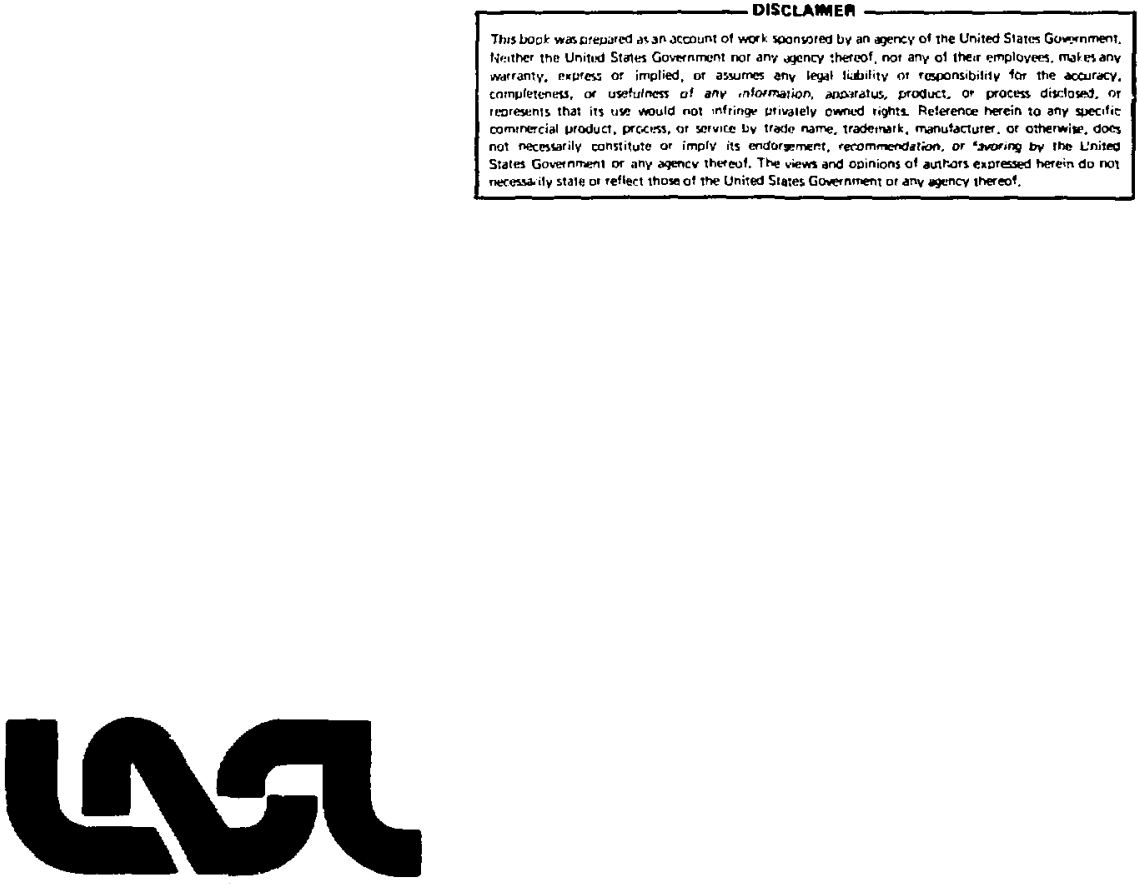
TABLE OF CONTENTS

CHAPTER

PAGE

I. INTRODUCTION AND BACKGROUNDD ................. 1

1.1 The Monte Carlo Method of Radiation Transport

Simulation--An Overview. .............. 1

1.2 Monte Carlo Estimation and Statistical Error . . . . . 9

1.3 Variance Reduction by Importance Sampling. . . . . . . 12

1.4 Splitting as a Form of Importance Sampling . . . . . . 16

1.5 Statement of the Problem . . . . . . . . . . 21

1.6 Organization of the Dissertation ........... 25

II. PREVIOUS STUDIES AND RELATED WORK . . . . . . . . . . . . 27

2.1 Work of Everett and Cashwell on the Purely Absorbing Slab . . . . . . . . . . . . . . 27

2.2 Analysis of Error in Monte Carlo Calculations . . . . 40

III. NATURE AND SCOPE OF THE PROBLEM TO BE STUDIED . . . . . . 45

3.1 Defining the Characteristics of the Transport Mode1 .. . . . . . . . . . . . . . . . 45

3.2 Nature of the Analysis to be Applied to the Problem .. . . . . . . . . . . . . . . 4 46

IV. THE CASE OF A SINGLE SPLITTING SURFACE . . . . . . . . 51

4.1 Deterministic Prediction of Sample Variance . . . . 52

4.1.1 Derivation of the Integra1 r-th Moment Equations $(r=1,2)$ for the Slab Transmission Score................ 53

4.1.2 Conversion to Integro-Differential Form .... 75

4.2 Deterministic Prediction of Time per Particle

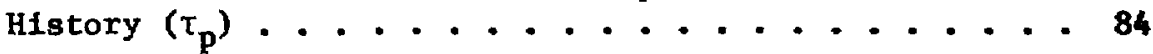

4.2.1 Derivation of First Moment Equation for $T_{p}$. . 84

4.2.2 Conversion to Integro-Differential For .... 93 
4.3 Solution to the Equations by Employing

Discrete Ordinates $\left(S_{n}\right)$ Technique ........... 98

4.3.1 Equations as Adjoint Formulations of

the Boltzmann Transport Equation . . . . . . 99

4.3.2 The ONETRAN $\left(S_{n}\right)$ Radiation Transport Code . . 100

4.3.3 Required Modifications to ONETRAN ....... 103

4.4 Numerical $\left(S_{n}\right)$ Study for the Case of a Single

Splitting Surface................. 105

4.4.1 Overview of the Study ............ 105

4.4.2 Monte Carlo/ $\mathrm{S}_{\mathrm{n}}$ Comparisons . . . . . . . 106

4.4.3 Single-Surface Results ............ 118

V. THE CASE OF MULTIPLE SPLITTING SURFACES . . . . . . . . . 1:3

5.1 Sample Variance, $\sigma_{s}{ }^{2}$. . . . . . . . . . . . . 133

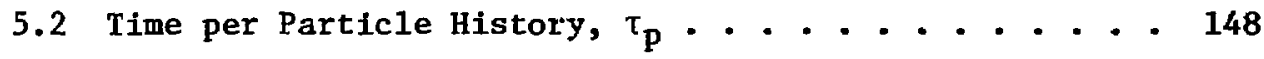

5.3 Numerical $\left(S_{n}\right)$ Study for the Case Involving

5.3.1 Overview of the Study ............. 160

5.3.2 Monte Carlo/s $\mathrm{s}_{\mathrm{n}}$ Comparisons ........... 161

5.3.3 Optimization of Splitting Surface
Locations . . . . . . . . . . 166

5.3.3.a Plane Normal Source .......... 167

5.3.3.b Isotropic Planar Source . . . . . . 167

5.4 Comparison of Optimal vs. Heuristic Placement

of Surfaces . . . . . . . . . . . . . . . 174

5.5 Comparison to Results from Standard Production

Monte Car1o Code (MCNP) . . . . . . . . . . . . 181 
VT. CONCLUSIONS AND RECOMMENDATIONS . . . . . . . . . . . 184

6.1 Conclustons . . . . . . . . . . . . . 184

6.2 Recommendations for Future Work . . . . . . . 187

REFERENCES .......................... 191

APPENDICES

A. FORMAI MODTFICATION OF TRANSPORT KERNEL DUE TO SPLITTING •

B. DERIVATION OF THE INTEGRAL :IOMENT EQUATIONS FOR THE MONTE CARLO SLAB TRANSMTSSION SCORE (SINGLE SPLITTING SURFACE)

B.1 The Integral Moment Equations $(r=1,2) \quad . . . . . .197$

B.2 Conditions at the Splitting Surface . . . . . . . 212

C. DERIVATION OF THE INTEGRAL (FIRST MOMENT) EQUATION FOR TIME PER PARTICLE HISTORY (SINGLE SPLITTING SURFACE) . . . . . . . . . . . . . . . . . 218

c.1 The First Moment Integral Equations for $\tau_{p}$. . . . . 218

C.2 Discontinuity Condition at $z=z_{8}$. . . . . . . . 221

D. WEIGHT SEPARABILITY IN THE $r$-th MOMENTS OF THE

SLAB TRANSMISSION SCORE ................ 226

E. THE ADJOINT DIFFERENTIAL TRANSPORT OPERATOR . . . . . . . 228

F. FORTRAN CODE LISTING FOR MCS1 . . . . . . . . . . . . 231

G. FORTRAN CODE LISTING FOR COSTFT . . . . . . . . . . 237

H. MULTIPLE SURFACE DERIVATIONS (HOMENTS OF SLAB TRANSMISSION SCORE) ................. 246

H.1 The First Moment: $(r=1)$. . . . . . . . . . 252

H.2 The Second Moment: $(r=2)$. . . . . . . . 256

I. MULTIPLE SURFACE DERIVATIONS (TIME PER PARTICLE HISTORY .......................... 264 


\section{LIST OF TABLES}

TABLE

PAGE

1 MCS1 Event Timing Sumnary . . . . . . . . . . . 111

2 Monte Carlo/s Comparisons $(\Sigma=3.0 \mathrm{mfp}) . . . . . . .113$

3 Monte Car1o/s Comparisons ( $\mathrm{D}=5.0 \mathrm{mfp})$. . . . . . 114

4 Monte Carlo/s ${ }_{n}$ Comparisons $(D=10.0 \mathrm{mfp}) . . . . . . .115$

$5 \quad$ Optimal Single-Surface Splitting Conditions . . . . . 132

6 MCSN Event Timing Summaries.............. 163

7 Monte Carlo/s ${ }_{n}$ Comparisons: Plane Normal Source . . . 164

8 Monte Carlo/s ${ }_{\mathbf{n}}$ Comparisons: Isotropic Source . . . . 165

9 Comparison of Several Cases ( $D=5.0 \mathrm{mf}$;

$\left.\Sigma_{\mathrm{S}} / \Sigma_{\mathrm{T}}=0.10\right) . . . . . . . . . . . . . . . .175$

10 Computing Costs Associated with Rule-of-Thumb

Splitting... . . . . . . . . . . . . 177

11 Cost Comparison: One vs. Two Splitting Surfaces

$\left(D=10.0 \mathrm{mfp} ; w_{s}=0.10\right) . . . . . . . . . .170$

12 Predicted vs. Stochastic (MCNP) Values of

Sample Variance ................ 183 


\section{LIST OF FIGURES}

FIGURE

PAGE

1 Major Operations in a Monte Carlo Calculation ......

2 Flow Chart Indicating the Frocessing of Geometry Splitting/Russian Roulette in a Typical Monte Carlo

Calculation . . . . . . . . . . . . . . .

3 Transport Model Used as Basis for Everett and Cashwell Analysis . . . . . . . . . . . . . . .

4 Verification of Everett and Cashwell Analysis:

Cost Function vs. Splitting Surface Location . . . . .

Behavior of $\tau_{p}$ and $\sigma_{s}{ }^{2}$ with Splitting Surface

Location . . . . . . . . . . . . . 34

6 Example of Inefficient Splitting in Thin Slabs . . . . 37

7 Cost Function vs. Splitting Surface Location

$(D=3.0 \mathrm{mfp} ; \mathrm{m}=2,3,5,7,10) \ldots . . . . . .$.

8 Transport Model Used as Basis for Analysis

(Isotropic Scattering Included) . . . . . . . . . .

9 Possible Next Events, Given a Particle Begins

its Next Flight to the Left of the Splitting

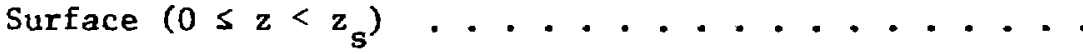

10 Deposition of Total Score s, Given the Particle

Begins its Next Flight at $z\left(0 \leq z<z_{s}\right)$....... 60

11 Possible Next Events, Given a Particle Begins its

Next Flight to the Right of the Splitting Surface

$\left(z_{s}<z \leq D\right)$. . . . . . . . . . . . . . . .

12 Deposition of Total Score s, Given the Particle

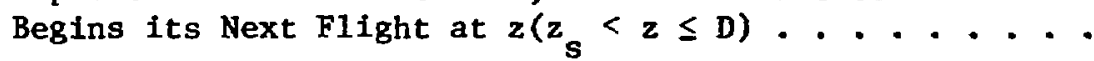

13 Possible Next Events, Given a Particle Begins

its Next Flight at the Splitting Surface $\left(z=z_{s}\right) .$. .

14 Deposition of Total Score s, Given the Particle Begins (Continues) its Flight at the Splitting 
15 Flowchart for Special-Purpose Monte Carlo Code MCS1 ................... 108-ilo

16 Cost Function vs. Splitting Surface Location $\left(D=5.0 \mathrm{mfp}, \mathrm{w}_{\mathrm{s}}=0.50, \mathrm{~m}=2\right) \ldots . . . . . . .117$

17-28 Cost vs. Splitting Surface Location (Twelve Cases: $w_{S}=0.10,0.50,0.90$ for $\mathrm{D}=2.0,3.0$, $5.0,10.0 \mathrm{mfp}) . . .+. . . . . . . . . . . .119-130$

29 Geometry Involving Multiple Splitting Surfaces . . . . 134

30 Next Events for a Particle Starting:

(a) in Region 1, and (b) at $z=z_{1}$......... 139

31 Next Events for a Particle Starting:

(a) in Region $i$, and (b) at $z=z_{i}$.......... 140

32 Next Events for a Particle Starting:

(a) at $z=z_{N}$, and (b) in Region $N+1 \ldots 141$

33 Cost vs. $\bar{z}_{2}$ (Plane Normal Source; $\left.z_{1}=1.0 \mathrm{mfp}\right) . . .168$

34 Cost vs. $\bar{z}_{2}$ (Plane Normal Source; $\left.z_{1}=2.50 \mathrm{mfp}\right) \quad$. . 169

35 Cost vs. $\bar{z}_{2}$ (Plane Normal Scurce; $z_{1}=4.00 \mathrm{mfp}$ ) . . 170

36 Cost vs $\bar{z}_{2}$ (Isotropic Source; $z_{1}=2.50 \mathrm{mfp}$ ) . . . . 173

A-1 Modification of Transport Kernel c(s)

Due to Splitting . . . . . . . . . . . . . 193 


\section{LIST OF SYMBOLS}

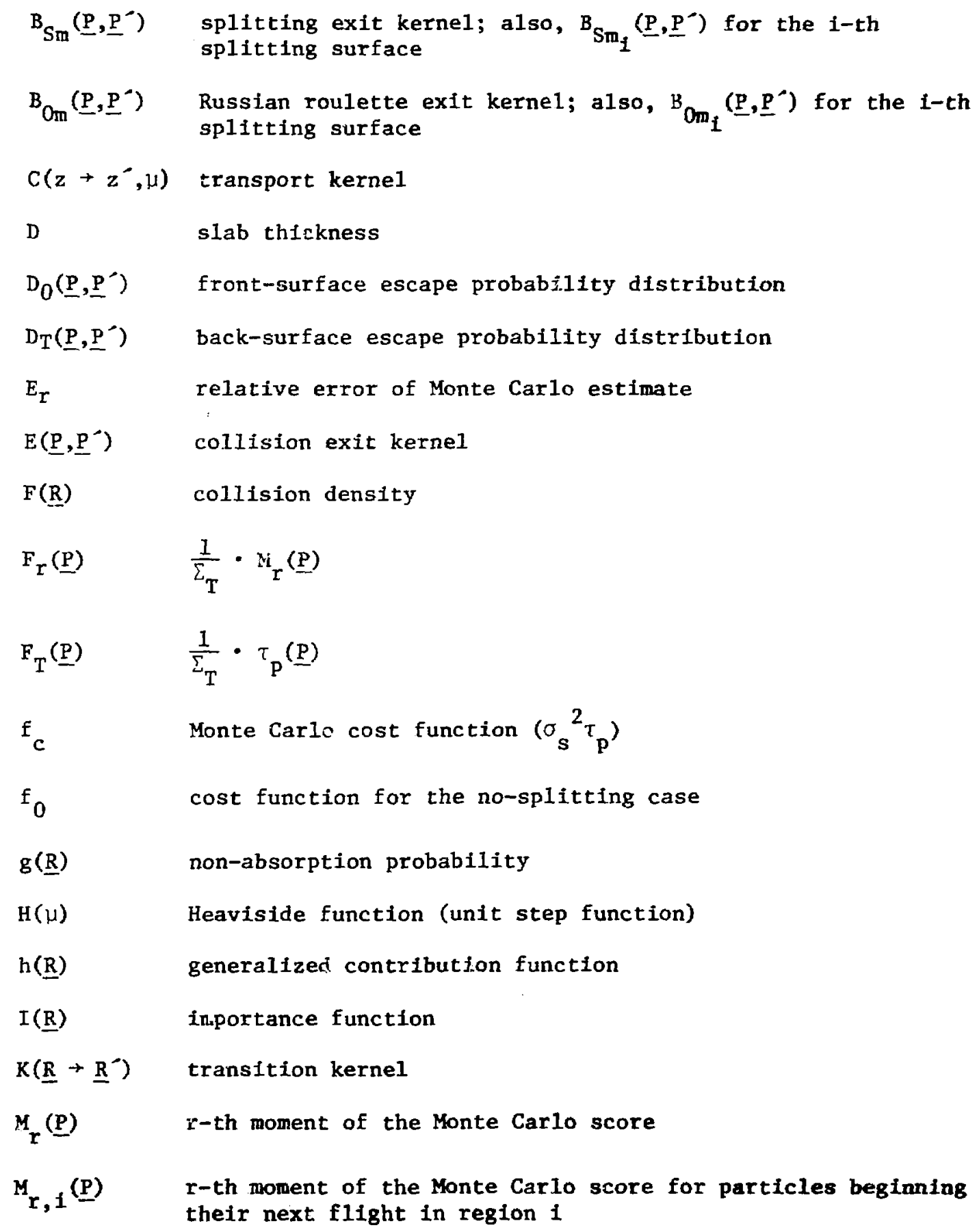




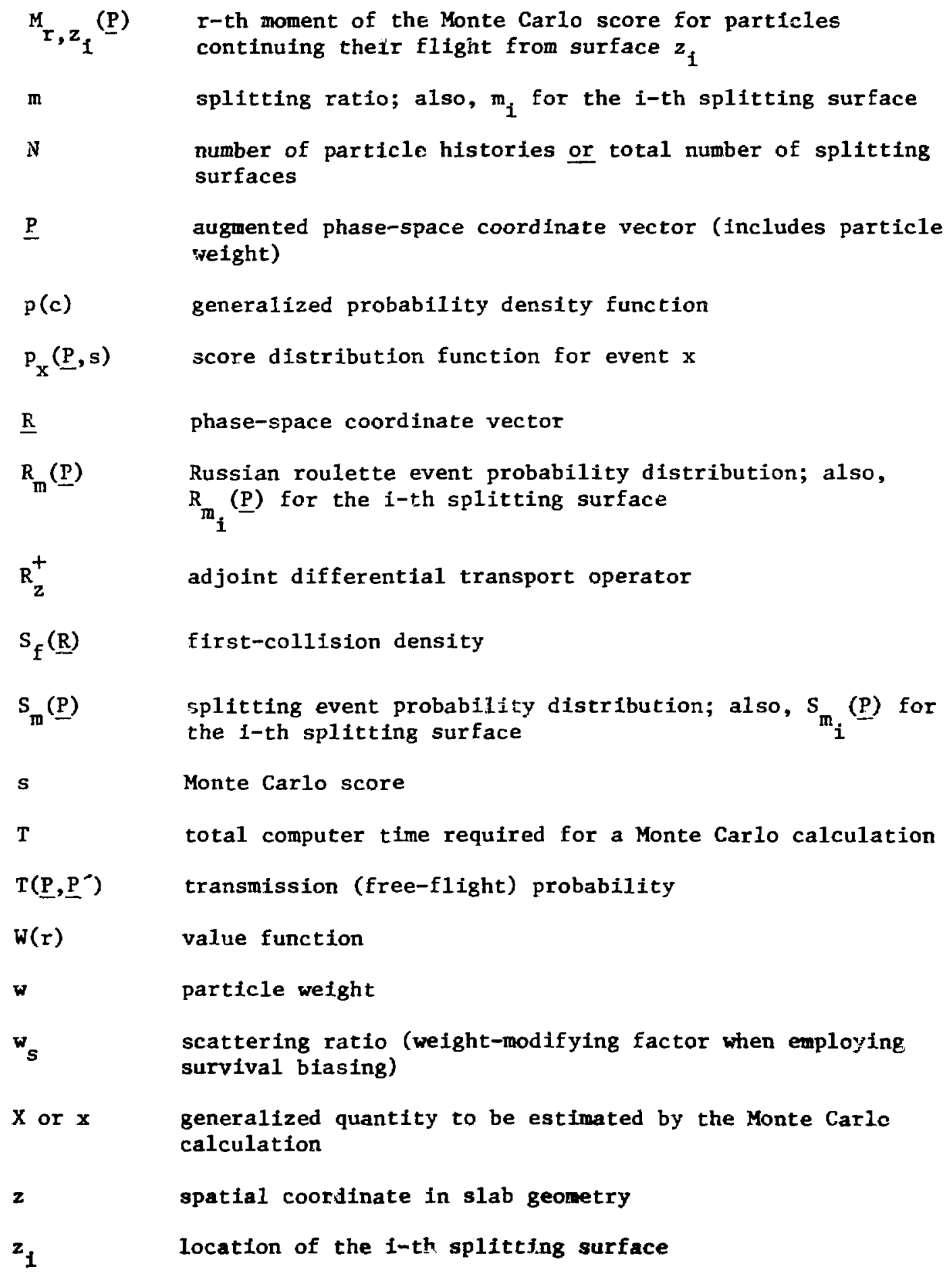




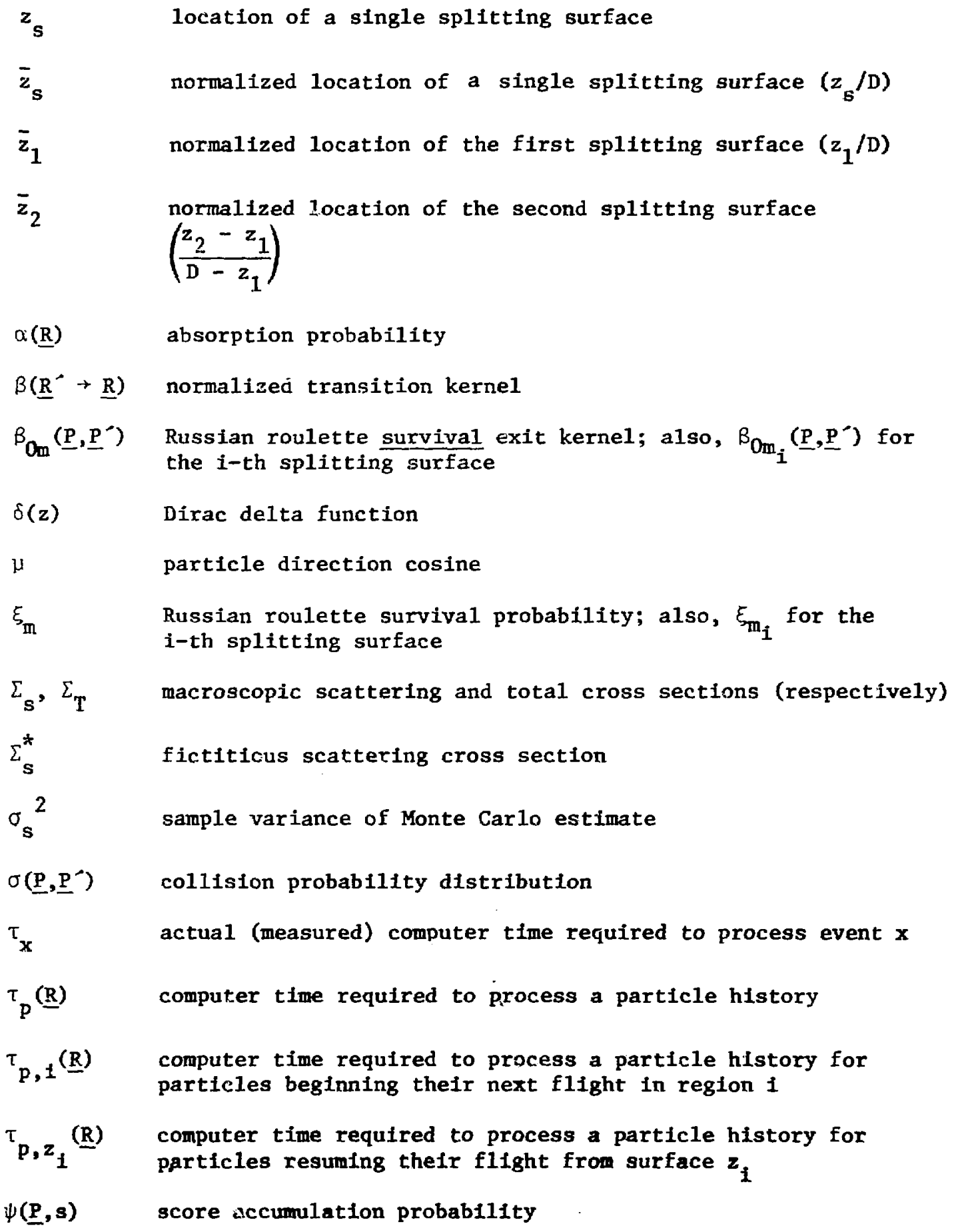


$\psi_{i}(\underline{P}, s) \quad$ score accumulation probability for particles beginning their next flight in region $i$

$\psi_{z}(\underline{P}, s) \quad$ score accumulation probability for particles resuming their $z_{i}$ flight from surface $z_{i}$ 
MINIMIZING THE COST OF SPLITTING IN MONTE CARLO

RADIATION TRANSPORT SIMULATION

by

Raymond John Juzaitis

ABSTRACT

A deterministic analysis of the computational cost associated with geometric splitting/Russian roulette In Monte Carlo radiation transport calculations to presented. Appropriate integro-differential equations are developed for the first and second moments of the Monte Carlo tally as we11 as time per particle history, given that splitting with Russian roulette takes place at one (or several) internal, irfaces of the geometry. The equations are solved using a standard $S_{n}$ (discrete ordinates) solution technique, allowing for the prediction of computer cost (formulated as the product of sample varlance and time per particle history, $\sigma_{g}^{2} \tau_{p}$ ) assoclated with a giveis set of splitting paraneters. Optimu splitting surface locations and splitting ratios are determined. Benefits of such en analysis are particularly noteworthy for transport problens in which splitting is apt to be extensively employed (e.g., deep penetration calculations). 


\section{CHAPTER I}

\section{INTRODUCTION AND BACKGROUND}

The sections comprising this introductory chapter serve to provide a very general overview of the Monte Carlo computational method. Varjance reduction in Monte Carlo calculations is discussed, and the use of splitting as a variance, reduction device is addressed specifically. The information in this chapter is presented solely to provide the proper context for understanding the particular nature and goals of this dissertation. For a more detailed discussion of the Monte Carlo method, the reader is directed to several other comprehensive referevices. $1,2,3$

\subsection{The Monte Carlo Method of Radiation Transport Simulation - an} Overview

The Monte Carlo method for solving radiation transport problems has developed considerably since its introduction at the Los Alamos Scientific Laboratory during World War II. Because it is essentially a method of statistical trials, Monte Carlo is highly computer intensiv.. and its popularity as a viable calculational tool is quite dependent on the evolutionary level of computer technology. Thus, whereas in the 1950 's and early 1960's the method was decried as a "method of last resort" to be used "only when no other method is available," 4 nevertheless by tive late $1970^{\circ}$ 's it had gained broad popularity in treating radiation transport problems. This occurred mainly due to the development of better, faster computers with enhanced memory capacity (for example, the CDC-7600), as well as the implementation of efficient 
variance reduction techniques In the Honte Carlo calculations themselves. The need for detailed solutions to problems, such as those involving complicated three-dimensional geometry, calculation of resonance escape probabilities and self-shielding effects, strong localized absorption, or mixed streaming/diffusion effects (to name just a few) has in many cases made Monte Carlo a more viable calculational tool than the more standard numerical techniques which normally suffer from various degrees of simplification.

With standard deterministic methods $\left(S_{n}\right.$, for example), an extensive, global description of the radiation field is calculated by solving the Boltzmann integro-differential transport equation for the radiation flux. Of course, the solution is obtained numerically, usually based on an appropriate level of simplification either of the transport equation itself or else in the transport model being considered.

On the other hand, the solutions to radiation transport problems by Monte Carlo are constructed by a random sampling of elementary statistical processes, all of which are described by appropriate probability distributions and based on the best cross-section data available. The inceractions of radiation (neutrons or gamma rays) passing through matter are stochastic in nature. The modeling of these realworld processes in the course of a calculation by selecting values for stochastic variables from unbiased probability densities results in an analog (direct simulation) calculation. Monte Carlo, then, is merely a stochastic experiment performed on a digital computer as an analog to the physical particle transport process. The goal of this numerical 
experiment is to obtain statistically valid estimates of certain quantities which depend in some way on the radiation field existing in a medium.

Particles are followed collision by collision from "he source to eventual termination. Since only neutral particles are being considered here, they are not affected by any significant long-range forces; hence, they travel between collisions at constant velocity for distances that are distributed statistically. Changes in energy and direction of travel occur only at collision points. Moreover, at collision sites the interaction physics are simulated in detail, and in the general case some type of scattering, multiplication, or capture may be candidate events. Termination of a history may be caused by absorption in the medium or by the particle attaining phase-space coordinates (for example, position, direction, energy) outside the range of interest for the given transport problem.

At any point during its history, a particle is totally characterized by its phase-space parameters. The seven-dimensional phase-space vector is comprised of (in general) three Euclidean spatial coordinates, two independent directional coordinates, the kinetic energy of the particle, and time. These phase-space parameters are sufficient to determine the probabilistic behavior of the particle in any situation it may encounter during its history. Monte Carlo thus generates random walks in phase space. From the "point of view" of the computer, the random walk is merely a succession of phase-space vectors. Formally, it is sometimes useful to consider the Monte Cario calculation as simulating a Markov process. ${ }^{5}$ The defining feature of a Markov process 
is that the probability of state-to-state transition depends only on the current state and not on the previous history of the system. The physical (neutral) particle transport process follows this Markovian behavior--a particle proceeds from source to termination through a series of points in frase space (states), i.e., the points corresponding to particle-medium interaction sites. A transition in phase space is determined only by the phase-space coordinates at the current collision site, not on the previous history of the particle. Monte Carlo thus models a terminating Markov chain.

During the execution of the random walk, a particle may contribute to one or more tallies, i.e., quantities of interest used to estimate current, flux, heating, etc. The total score over the history in a particular ially category is one particle's estimate of the corresponding functional being evaluated.

When random walks generate collisions in phase space, a solution to the Monte Carlo transport problem may be formally expressed as an integral quantity involving some contribution function $h(\underline{R})$ weighted by the collision density $F(\underline{R}) .1$ Thus,

$$
X=\int h(\underline{R}) F(\underline{R}) d \underline{R} .
$$

Here $\mathrm{X}$ is the functional to be evaluated by the Monte Carlo calculation. The above integral is taken over all of phase space, and $\underline{\mathrm{R}}$ represents generalized phase-space coordinates.

Carter and Cashwell ${ }^{1}$ derive a formal expression for the Monte Carlo solution by first writing a simple version of the integral transport equation for the collision density: 


$$
F(\underline{R})=\int K\left(\underline{R}^{\prime} \rightarrow \underline{R}\right) F\left(\underline{R}^{\prime}\right) d \underline{R}^{\prime}+S_{f}(\underline{R}),
$$

where $S_{f}(\underline{R})$ represents the first-collision density and $K\left(\underline{R}^{\prime} \rightarrow \underline{R}\right)$ is the transfer kernel from $\underline{R}^{\prime}$ to $\underline{R}$. Thus, the collision density at $\underline{R}$ is made up of particles coming into collision at $\underline{\mathrm{R}}$ after having suffered $\mathrm{a}$ previous collision at $\underline{\mathrm{R}}^{\prime}$, as well as particles coming into their first collision at $\underline{R}$ from the source. An expression for the collision density at $\underline{\mathrm{R}}^{-}$[that is, $\left.\mathrm{F}\left(\underline{\mathrm{R}}^{-}\right)\right]$may be written analogously to $\mathrm{Eq}$. (1.1.1a) in terms of $F\left(\underline{R}^{\prime \prime}\right)$, and this may be substituted in $\mathrm{Eq} \cdot(1.1 .1 \mathrm{a})$. The procedure may be repeated for the collision density in each successive integrand, ultimately resulting in an infinite-series representation for $F(\underline{R})$. This Neumann series may then be substituted back into Eq. (1.1.1) to yield an expression for the Monte Carlo solution:

$$
\begin{aligned}
X= & \sum_{n=0}^{\infty}\left[\int \cdots \left(S_{f}\left(\underline{R}_{0}\right) g\left(\underline{R}_{0}\right) \beta\left(\underline{R}_{0} \rightarrow \underline{R}_{1}\right) g\left(\underline{R}_{1}\right) \beta\left(\underline{R}_{1} \rightarrow \underline{R}_{2}\right)\right.\right. \\
& \left.\cdots g\left(\underline{R}_{n-1}\right) \beta\left(\underline{R}_{n-1} \rightarrow \underline{R}_{n}\right) \alpha\left(\underline{R}_{n}\right) \xi\left(\underline{R}_{0}, \underline{R}_{1}, \ldots, \underline{R}_{n}\right) d \underline{R}_{0} \cdots \underline{R}_{n}\right],
\end{aligned}
$$

where

$$
\begin{aligned}
\alpha\left(\underline{R}_{n}\right)= & \text { absorption probability at } \underline{R}_{n}, \\
g\left(\underline{R}_{n}\right)= & \text { non-absorption probability }\left[=1-\alpha\left(\underline{R}_{n}\right)\right], \\
B\left(\underline{R}_{n-1}+\underline{R}_{n}\right)= & \text { normalized transition kernei; i.e., the } \\
& \text { probability of entering the next collision } \\
& \text { at } \underline{R}_{n} \text {, having entered the present colli- } \\
& \text { sion at } \underline{R}_{n-1} .
\end{aligned}
$$

In writing $\mathrm{Eq}$. (1.1.2), the transfer kernel $\mathrm{K}$ has been factored into 
constituent probability functions and several factors have been combined with $h\left(\underline{R}_{n}\right)$ in expressing $\xi$, the Monte Carlo score.

The leading part of the integrand in Eq. (I.1.2) expresses the probability for a particular random walk. The probability of the particle experiencing its first collision in $\underline{R}_{0}$ about $\underline{R}_{0}$ is expressed by $S_{f}\left(\underline{R}_{0}\right) d R_{0}$. Subsequent factors represent the probability of a series of intermediate non-absorbing collision events. The particle is finally absorbed at $\underline{R}_{n}$ with probability $\alpha\left(\underline{R}_{n}\right)$. The score (or tally) resulting from a particular random walk upon termination at $\underline{R}_{\mathrm{n}}$ is expressed as $\xi\left(\underline{R}_{0}, \underline{R}_{1}, \ldots, \underline{R}_{n}\right)$. The tally contribution of a particular history, $\xi$, is a random variable defined on the space of all random walks. The expected value of $\xi$ over the distribution of random walks is shown by Eq. (1.1.2) to be equal to the Monte Carlo estimate of interest; i.e., $\langle\xi\rangle=\mathrm{X}$. Although the Formulation of $\mathrm{Eq} \cdot(1.1 .2)$ implies a last-event estimator, the foregoing statement generalizes to a11 estimators.

As can be seen from the discussion above, the analytical essence of Monte Carlo is formally embodied in an analog probability model which is based on the physical behavior of neutrons as described by the Boltzmann transport equation. (As will be seen, this notion underlies the theoretical considerations upon which the work presented in this dissertation is based.) Furthermore, the result of a Monte Carlo calculation, namely the expected value of an appropriate estimator over all random walks, effectively represents (in an integral sense) the Neumann series solution to the integral transport equation. 
Nevertheless, apart from analysis, there is no need to resort to mathematical formalism in order to understand the practical nature of the Monte Carlo method. The main elements of the calculation are depicted graphically by referring to a generalized flow chart $^{6}$ (see Fig. 1) and the following corresponding text:

(1) By sampling the appropriate source distributions, the initial position, direction, energy of the primary particle are set.

(2) The distance to the first collision $D_{c}$ is sampled, and the distance to the nearest surface $D_{s}$ along the line of flight is calculated.

(3) If $D_{c}<D_{S}$, a collision results, and the neutron is advanced to the collision site. Otherwise, the particle escapes to another region along the same line of flight without interaction; it is advanced to the surface.

(4) If a collision occurs, appropriate sampling allows for determination of the isotope undergoing interaction as well as the particular reaction tyra. The particle's phase-space parameters are then updated and tracking continues ("tracking" here refers io following the random walk of the particle).

(5) If a surface is intersected, the cell to be entered is determined. Geometric splitting or Russian roulette (see section 1.4) may take place. Any extra subparticles produced by the splitting event are stored in the bank. ("Sub-particles" here refer to the progeny resulting from a splitting event, the sum of whose weights add up to the weight of the original particle which was split.) Tracking then continues in the new cell.

Tracking continues until some category of termination is encountered: escape of the overall geometry, geometric Russian roulette, or cutoff in weight, energy, or time. The chart in Fig. 1 implies the treatment of absorption by survival biasing; that is the weighting-out of absorptions by appropriately reducing the particle's weight at each collision. In this case, an appropriate weight-cutoff gave may be played. 


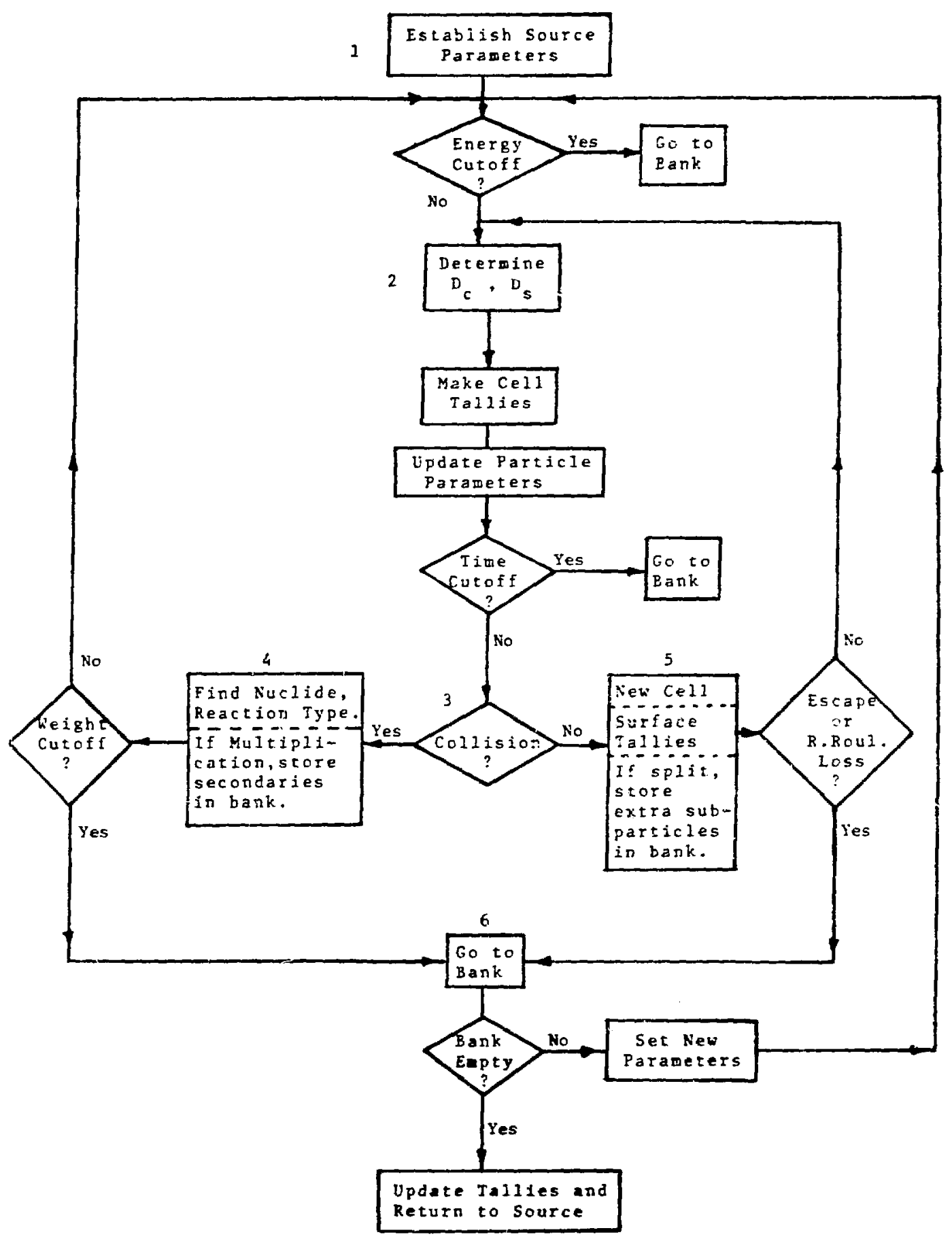

Figure 1. Major Uperations in a Monte Carlo Calculation. 
(6) After termination of the primary particle, all secondaries or sub-particles which were produced by the primary (elther by multiplication events or splitting) are retrieved from the bank and tracked. Otherwise (if the bank is empty), the history is complete.

It should be noted that during the tracking process as illustrated in Fig. 1, scoring may take place both within cells (cell tallies) or on surfaces (surface tallies).

Thus, all formalism aside, the intrinsic beauty of the Monte Carlo method derives from its conceptual simplicity as a direct simulation of the physical particle transport process.

\subsection{Monte Carlo Estimation and Statistical Error}

As we have seen, the tally score resulting from a given rancom walk is a random variable in the sense that a real-valued function is assigned to an event (or outcore) of the sample space (in this case, of random walks). If an appropriate, unbiased estimator is used for scoring purposes, the expected value of the tally score over all random walks constitutes the Monte Carlo estimate of the given quantity of interest. Quantities such as average flux over a given region, flux at a point, heating in a given region, total number fof collisions in a certain cell, neutron curnent crossing specified boindaries, etc., may be estimated in this manner.

The arithmetic mean of the score over $\mathrm{N}$ independent particle histories is expressed as

$$
\bar{\xi}=\frac{1}{N} \sum_{i=1}^{N} \xi_{i} \approx\langle\xi\rangle,
$$


and this sample mean is used as an approximation to the true mean, or expected value. (The expected value $\langle\xi\rangle$ is the first mont of $\xi$ with respect to the probability density of randou walks.)

Corresponding to the mean sample estimate is an associated probabilistic error arising fron the statistical behavior of the particles. It is of prime importance to establish a quantitative error bound, 1.e., a confidence level on the estimate in question. For this reason the Central Limft Theorem plays an important role in Monte Carlo Analysis. ${ }^{1,3}$ Briefly stated, if $\xi_{1}, \xi_{2}, \ldots, \xi_{\mathrm{y}}$, is a series of 1 independent random variables (distributed according to the same probability distribution), and these random variables have a comon true mean $\langle\xi\rangle$ and standard deviation $\sigma_{s}$, then the sample (arithmetic) mean is normally distributed about the true mean as the sample size increases to infinity in the sense that

$$
\lim _{N \rightarrow \infty} \operatorname{Prob}\left\{a \leq\left[\frac{\bar{\xi}-\langle\xi\rangle}{\sigma_{s} / \sqrt{N}}\right] \leq b\right\}=\frac{1}{\sqrt{2 \pi}} \int_{a}^{b} e^{-t^{2} / 2} d t
$$

The Central Limit Theoren allows for the calculation of the probability that $\bar{\xi}$ will deviate from $\langle\xi\rangle$ by wore than a given wount. It permits one to quantify meaningfully the uncertainty associated with a Monte Carlo estiante.

One of the most inportant measures of central tendency in statistical distributions is the variance. In order to provide practlcal significance to the Central Linit Theorem, it is necessary to be able to calculate the variance of a Monte Carlo ample estinate, 
$\sigma_{s}^{2}$. Formally, if $\mathrm{p}(\mathrm{c})$ denotes the probability distribution of random walks $c$, then the variance is defined as the second central moment of the random variable, that is, the second mowent about the mean: 3

$$
\sigma_{s}^{2}(\xi) \equiv \int[\xi(c)-\langle\xi\rangle]^{2} p(c) d c=\left\langle\xi^{2}\right\rangle-\langle\xi\rangle^{2} .
$$

As with the mean, the sample variance is usually determined approximately and in terms of the first and second sample moments. In fact, it can be shown ${ }^{7}$ that a consistent and unblased estimate of the variance may be calculated as follows:

$$
\bar{\sigma}_{s}^{2}(\xi)=\frac{N}{N-I}\left[\frac{1}{N} \sum_{i=1}^{N} \xi_{i}^{2}-\left(\frac{1}{N} \sum_{i=1}^{N} \xi_{i}\right)^{2}\right]
$$

where, for large sample sizes, it is valid to consider the leading factor as unity.

The expression for the variance given in Eq. (1.2.4) formulates the varfance of $\mathrm{N}$ sample estimates about their mean. It is also of interest to calculate the variance of the sample mean about the true nean which may be expresseci as

$$
\sigma^{2}(\bar{\xi})=\frac{\sigma_{s}^{2}(\xi)}{N}
$$

and this allows for the formulation of the relative error of a Nonte Carlo estinate as follows: 


$$
E_{\mathrm{r}}=\frac{\sigma(\bar{\xi})}{\langle\xi\rangle}=\frac{\sigma_{\mathrm{s}}(\xi)}{\sqrt{\mathbb{N}}\langle\xi\rangle} \approx \frac{1}{\sqrt{\bar{N}}}\left(\frac{\bar{\sigma}_{\mathrm{s}}(\xi)}{\bar{\xi}}\right) .
$$

The latter quantity is used in most applications as the prime statistical indicater for Monte Carlo results.

Obviously, it is desirable to calculate a quantity with as low a relative error as possible. Due to the inverse square-root dependence on the number of histories, efforts to reduce $E_{r}$ by increasing $N$ are met with diminishing returns as $\mathrm{N}$ becomes large. The key practical way for reducing the relative error associated with Monte Carlo estimates, however, is to modify the calculation in order to yield results which are accompanied by a lower sample variance $\bar{\sigma}_{s}{ }^{2}$.

Nevertheless, as will be emphasized time and again in this dissertation, reducing the relative error is enly a partial solution to the problem of ensuring efficiency of the Monte Carlo calculation. The real goal is to produce an estimate with a relailive error that is as small as possible for a fixed amount of computer time, or, in other words, to achieve a prescribed relative error with a mininam of computer time.

\subsection{Variance Reduction by Importance Sampling}

If a Monte Carlo calculation were to result in a series of estimating random variables (scores) which were rloser in magnitude to thetr mean value, the sample variance would be reduced. This, indeed, is the goal of all variance reduction techniques. Many such modifications to the Monte Carlo calculation are employed. A few examples are source biasing, the use of inproved estimates involving expected values, 
and survival biasing, i.e., the weighting-out of absorptions. However, the method important to this work and upon which emphasis will now be placed ts a broad class of techniques known as importance sampling. In section 1.1 , it was tacitiy assumed that the Monte Carlo calculation being considered was a direct simulation, or analog calcu1ation; that is, the probability deissities being sampled in the course of the random walk correspond to the exact source and transition distributions as dicta:ed by the physics of the particle transport process. When importance sampling is employed, however, sampling takes place from biased, or fictitious, density functions.

In most Monte Carlo transport problems, the nature of the functional being estimated is such that a great many (if not most) of the particle histories, when tracked in an analog manner, do not contribute at all to the estimation. This large number of zero-contributions has an adverse effect on the sample variance. Probably the prime examples of such behavior are radiation transport problems involving deep penetration.

As the name implies, importance sampling is a biased sampling scheme which emphasizes the more important regions of the problem. Specifically, the analog transition probabilities are disterted in a manner which preferentially transports particles to points in phase space from which significant contributions to a given Monte Carlo tally are expected. In other words, a greater number of particle histories results in some non-zero estimate of the functional. Correspondingly, the variance is reduced. 
Suppose that the fictitious density functions which are sampled in the course of such an altered scheme result from the appropriate weighting of the analog densities by an importance function $I(\underline{R})$. For example, recalling the transition probabilities alluded to in Eq. (1.1.2),

$$
\tilde{\beta}\left(\underline{R}_{i}+\underline{R}_{i+1}\right)=\beta\left(\underline{R}_{i} \rightarrow \underline{R}_{i+1}\right) \times\left[\frac{I\left(\underline{R}_{i+1}\right)}{I\left(\underline{R}_{i}\right)}\right] .
$$

Here $\widetilde{\beta}$ and $\beta$ are the biased and analog probabilities, respectively. The importance function $I(\underline{R})$ represents an approximation to the expected contribution to the tally of interest from a particle starting out at phase-space location $\underline{R}$. In fact, if the solution to the adjoint Boltzmann transport equation is used as the importance function in weighting the analog transition probability densities $\beta$ [as well as $\mathrm{S}_{\mathrm{f}}$ and $\alpha$; see Eq. (1.1.2)], it can be shown that a zero-variance estimate may result. 1 of course, the solution to the adjoint equation is just as difficult as the Monte Carlo problem under condsideration. For this reason. simplified importance functions which serve as approximations to the adjoint are used in most applications to modify the transition kernels.

Nevertheless, no matter what the exact form of the importance function 1s, a11 distortions to the analog probability densities must be accompanied by a sultable weight modification to the history score in order to preserve the unblased estimation of the functional in question. If $p(c)$ is again considered to be the generalfzed probabilfty 
density of random walks $c$, then the effects of importance sampling may be sumarized concisely. Recall [see Eq. (1.2.3)] that the variance of a Monte Carlo estimate may be formulated in terms of the first and second moments of the score:

$$
\sigma_{s}^{2}(\xi)=\int \xi^{2}(c) p(c) d c-\left[\int \xi(c) p(c) d c\right]^{2} .
$$

Now, if $\widetilde{p}(c)$ represents the modified probability density of random walks, the score $\xi(c)$ must be modified by an appropriate weight factor:

$$
\widetilde{\xi}(c)=\xi(c) \times W t=\xi(c) \times\left[\frac{p(c)}{\widetilde{p}(c)}\right] .
$$

The first and second moments of the altered score may then be formulated as follows: ${ }^{8}$

$$
\langle\widetilde{\xi}\rangle=\int \tilde{\xi}(c) \tilde{p}(c) \mathrm{d} c=\int \xi(c)\left[\frac{\mathrm{p}(c)}{\widetilde{\mathrm{p}}(\mathrm{c})}\right] \tilde{\mathrm{p}}(\mathrm{c}) \mathrm{d} c=\langle\xi\rangle,
$$

and

$$
\left\langle\tilde{\xi}^{2}\right\rangle=\int \tilde{\xi}^{2}(c) \tilde{p}(c) d c=\int \xi^{2}(c)\left[\frac{p(c)}{\tilde{p}(c)}\right] p(c) d c \neq\left\langle\xi^{2}\right\rangle .
$$

As is indicated by Eq. (1.3.4a), the first moment (expected value) of the score obtained from the altered sampling scheme is identical to the analog mean. On the other hand, the second moment corresponding to the altered scheme may be lower than the analog second monent if the weight factor $(p(c) / \tilde{p}(c))$ is less than unity whenever the contribution $\xi(c)$ Is significant. Note that trying to achieve zero variance inplies 
keeping the product $\tilde{\xi}=\xi \times\left(\frac{\mathrm{p}}{\widetilde{\mathrm{p}}}\right)$, hence the modified score, approximate1y constant (equal to the mean).

Employing importance sampling in a Monte Carls calculation results in the construction of a fictitious collision density which is not described by the Boltzmann transport equation. Nevertheless, if one interprets the particles being tracked as "carrying with them" the appropriate weight modifications (rather than saving all the weight factors until a score is made), then the expected weight density about to undergo a collision at $\underline{R}$ satisfies the transport equation as does the true analog collision density.

\subsection{Splitting as a Form of Importance Sampling}

The technique of splitting is probably one of the most widely employed variance reduction methods in Monte Carlo particle transport calculations. (Note that the concurrent implementation of Russian roulette, the counterpart of splitting, is implicitly considered in this discussion.) Owing to its conceptual simplicity, ease of implementation, and its effectiveness over a wide range of problems and broad range of implementation parameters, splitting has acquired the distinction of being a simple variance reduction technique to use, associated with little danger for ajuse. Unfortunately, possibly due to its straightforward applicability, splitting has received little academic attention, and formal studies/analyses of the technique are very scarce in the literature.

Splitting and Russian roulette modify the Monte Carlo calculation by ensuring that a proportionately greater amount of computational 
effort is spent on particles that enter regions of phase space from which there is a higher probability of contributing to the Monte Carlo tally. Although it is possible to discuss formally the implementation of splitting in phase space, conventional splitting/Russian roulette methods, e.g., as in the MCNP code, 9 are restricted to geometry and energy. Moreover, energy splitting and geometry splitting are invoked independently of each other. Only geometry splitting is considered in this work. The mechanics of the technique may be summarized as follows (with reference to Fig. 2).

The problem geometry may be divided into regions of varying importance (region importance being defined by the user as an input parameter). Splitting or Russian roulette is invoked when a particle is about to enter a new geometry cell. If the importance of the newlyentered cell is greater than that of the old cell, i.e., $I_{n+1}>I_{n}$, then the particle with an initial weight $w$ is split at the boundary between regions into a larger number $m$ of sub-particles according to $m=I_{n+1} / I_{n}$. (For the purpose of this work, $\mathrm{m}$ is considered to be an integer.) Each sub-particle has a reduced weight: $w^{\prime}=w \cdot\left(I_{n} / I_{n+1}\right) \cdot$ Thus, subparticles are particles of reduced weight which are formed at the splitting event. At the instant of the split, the sum of the weights of all sub-particles is equal to the weight of the parent particle; their phase-space parameters at this point are identical to those of the parent. This splitting procedure effectively gives a particle history an increased chance of accumulating via the sub-particles a (statistically weighted) contribution to the tally. On the other hand, if a partfcle enters a cell of less importance $\left(I_{n+1}<I_{n}\right)$, then it survives Russian roulette 


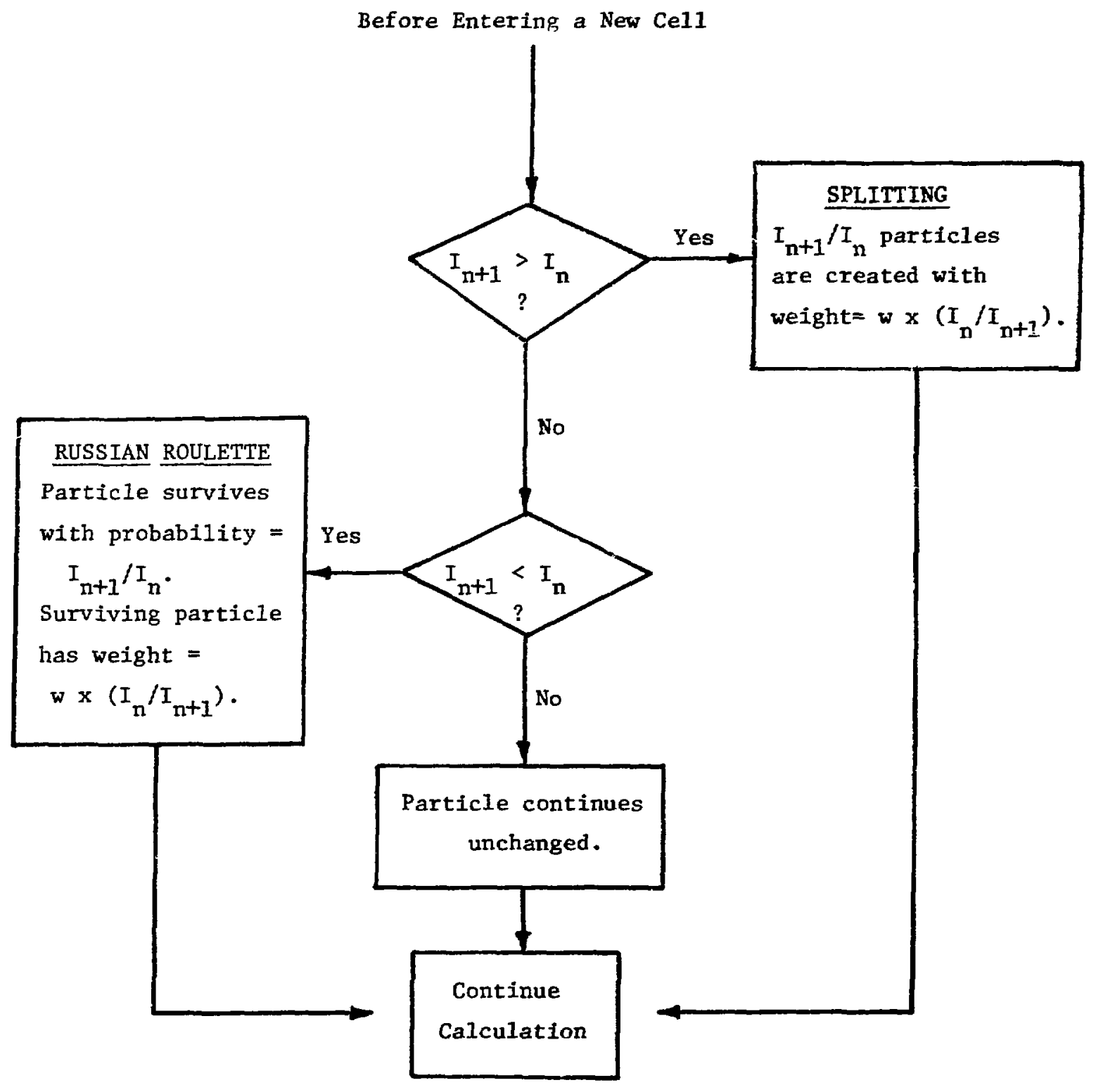

Figure 2. Flow Chart Indicating the Processing of Geometry Splitting/Russian Roulette in a Typical Monte Carlo Calculation. 
with a probability $I_{n+1} / I_{n}$. If the particle survives, its weight is enhanced: $w^{\prime}=w \cdot\left(I_{n} / I_{n+1}\right)$. This reduces the computer time that could be wasted on particles that will probably not contribute a score to the tally of interest but still keeps the calculation unbiased.

It is important to note the justification for the reduction in sample variance which accompanies splitting when this technique is used in Monte Carlo. The effect of splitting is to increase the sampling rate of points in phase space from which contributions to the tally are expected. Since a given random walk is apt to sample important regions more extensively, it is intuitively clear why splitting is considered a form of importance sampling. When particles enter important regions, their "success" is rewarded by splitting. The process gives each "1ucky" history, as it were, an extra chance to tally. The effect is to decrease the number of zero-contributions. Also, the implementation of geometry splitting in combination with Russian roulette allows (given there are no other weight-modifying processes) all particles in a given importance region to have the same weight--obviously having a favorable effect on the sample variance.

More specifically, though, splitting causes a reduction in variance by grouping the tally contributions of independent random walks closer together. This is demonstrated with a simple example involving a seniinfinite, purely ebsorbing slab with a normally-incident flux of particles. The tally of interest is the transmission through the slab. Assume a Monte Carlo sample size of 1000 histories, and the expected value for the transmission is $\overline{\mathrm{X}}=0.50$. 
Playing a purely analog Monte Carlo game (no splitting), each history can contribute only a 1 or 0 to the tally. The following sample estimate and variance may represent a possible outcome of the analog game:

$$
\begin{aligned}
& \overline{\mathrm{x}}=\frac{500 \times(1.0)+500 \times(0.0)}{1000}=0.50, \text { and } \\
& \bar{\sigma}_{s}{ }^{2}=\frac{500 \times(1.0)^{2}+500 \times(0.0)^{2}}{1000}-0.25=0.25 .
\end{aligned}
$$

However, if each particle is first split at the source into two subparticles (each of weight 1/2), then each history can score either 0 (if both sub-particles fail to traverse the slab), 1/2 (if one of the sub-particles traverses the slab), or 1 (if both particles score). The altered game may yield the following results:

$$
\begin{gathered}
\overline{\mathrm{x}}=\frac{250 \times(0.0)+500 \times(0.50)+250 \times(1.00)}{1000}=0.50, \text { and } \\
\bar{\sigma}_{s}{ }^{2}=\frac{250 \times(0.0)^{2}+500 \times(0.50)^{2}+250 \times(1.0)^{2}}{1000}-0.25=0.125 .
\end{gathered}
$$

The sample variance in the second game was reduced because of the reduction in the number of zero-contributions as well as the fact that the tally scores were grouped closer together than was possible in the analog case. Splitting thus allowed for the sharper peaking of the tally score distribution about the mean value. (Note that since the game with splitting also took twice as long to process, there really was no true cost benefit vis $a^{\prime}$ vis the analog game. Nevertheless, 
this simple example was inciuded only to show the favorable effect of splitting on sample variance.)

In concluding this section, it should be stated that due to the conceptual simplicity of the technique, the iormal (rather than practical or intuitive) characterization of splitting as an importance sampling device is elusive in the sense that analog probability densities should be weighted by an importance function to produce fictitious densities. However, Kalos et al., 10 have formally interpreted splitting as a modification of the transport kernel. The argument is outlined in Appendix A.

\subsection{Statement of the Problem}

Having discussed the concept and implementation of splitting (and Russian roulette) in Monte Carlo calculations, it is now possible to address the specific nature and purpose of the research work which comprises this dissertation.

Although splitting's general effectiveness, conceptual simplicity, and ease of implementation have resulted in its being dubbed as "easy to use, hard to abuse," unfortunately abuse is not impossible. For example, by failing to split enough, the sample size in important problem regions may drop so low that information may be lost in these regions. On the other hand, overuse of splitting may result in the wasting of valuable computer time due to the tracking of a huge number of sub-particles which do not aid proportionately in the reduction of variance. As with most other variance reduction devices, the implementation of splitting requires some a priori information regarding the 
specific values of importance sampling parameters. Unfortunately, too many times these parameters are defined by resorting to educated guesses, usually based on the experfence and/or insight of the user. Because of the lack of a more analytical basis for specifying these parameters, the implementation of such a variance reduction technique becomes more an art and less a science. 8

In the case of geometry splitting, the user is required to supply information in order to specify the splitting parameters, namely:

(1) how many splitting surfaces to employ, (2) where to locate them, and (3) what relative importances should be assigned to different regions. Sometimes the importance regions are already implicitly defined geometrically by the problem itself. More of ten than not, rough guesses must be made by the user (hopefully backed by experience) in the process of defining the splitting parameters. Luckily, splitting way be effective when such rough guesses are made. This is not always the case, however. As was noted above, misuse is possible.

of course, there are several heuristics (or rules of thumb) which may be employed by the user in order to help eliminate the guesswork involved in locating splitting surfaces. A few of these (which are not necessarily recommended) are listed below: 10,11

- Split by a factor of 2 for every mean free path traveled (defined at some average energy),

- Locate splitting surfaces at locations $a_{j}(j=1,2, \ldots)$ in a slab, chosen so that the probability of a particle reaching $a_{j}$ would be $2^{-j}$,

- Split by 2 whenever the particle flux falls by a factor of 2 . 
Macdonald, et al., 6,8 have addressed the problem of splittingparameter specification and have attempted to relieve the user of some of the a priori quantitative decisions that are required with regard to specifying splitting parameters by having the surface locations "learned" in the course of the calculation. In their work, phase-space coordinates are appropriately reduced and correlated to produce a feature space. Decision surfaces are then used to separate the feature space into importance classes. Using the techniques of pattern recognition, prototype particle histories are processed and splitting surfaces are learned in phase space. Such techniques, when implemented in a general-purpose Monte Carlo code, have had a favorable effect on the calculational cost in many cases. Nevertheless, the user is still required to supply the number of splitting surfaces to be learned as well as information needed to construct the feature space. Moreover, and most importantly, there are no assurances that the "teacher classification" of prototypes is performed so: 3 to result in the learning of the optimum surface locations.

The statement of the problem to be addressed by this dissertation may be formulated then quite simply. Generally speaking, it is desirable to place the selection of splitting parameters on a more sound analytical basis. The aim of this dissertation is to employ an appropriate numerical technique in order to deterministically predict the efficiency of a given set of splitting parameters in a lonte Carlo calculation. Given a fixed transport model, the goal is to ascertain the existence of and to quantify those splitting paraneters which lead to the most efficient calculacion of a Monte Carlo estiate. This 
includes finding the optimum locations for the surfaces as well as the optinum values for the importance ratios characterizing adjacent regions. Before proceeding further, it is important to define an appropriate quantilative indicator with which it is possible to judge the relative efficiency of a Monte Carlo calculation. A consideration only of the variance is simply not enough. A variance reduction method could be successfully implemented in a calculation but only at the expense of such coding complexity and so many additional calculations per particle history so as to render the calculation inefficient and impractical. For example, as was discussed in section 1.4 , splitting reduces the variance of a sample when compared to an analog calculation. However, the technique also introduces new operations requiring an extra amount of time per particle history. These extra operations include (for geometry splitting only):

1) performing the splitting and Russian roulette operations themselves and storing the new sub-particles produced by splitting;

2) an increased number of geometry-related operations due to the definition of new splitting surfaces which do not correspond to pre-existing geometric boundaries (thus an additional number of surface intersections and new geometry tracking calculations is involved); and

3) tracking of all the new sub-particles introduced as a result of splitting (including the extra tally updates involved).

A more appropriate measure of computational efficiency is the total computer time, involved in achieving a given relative error in the calculated estimate. The following expression may be derived ${ }^{12}$ for the total computer time $T$ : 


$$
T=\left[E_{r}^{2}\langle\xi>?]^{-1}\left(\sigma_{s}^{2} \tau_{p}\right)\right.
$$

Here $E_{r}=$ relative error to be achieved,

$$
\langle\xi\rangle=\text { expected value of the estimating random }
$$
variable (score),

$\sigma_{s}{ }^{2}=$ sample variance of the score, and

$\tau_{p}=$ mean computer time per particle history.

Since the factor $\left[E_{r}{ }^{2}<\xi>2\right]^{-1}$ is method-independent: whereas $\left(\sigma_{s}{ }^{2} \tau_{p}\right)$ depends on the method of calculation, it may be concluded that the latter factor is the correct indicator for the relative cost of a calculation. Thus, for the purposes of this dissertation, we will define the cost function as:

$$
f_{c} \equiv \sigma_{s}^{2} \tau_{p}
$$

This is the figure of merit which will be used for judging the costeffectiveness of a particular splitting scheme, i.e., in locating optimum splitting parameters. A reduction in sample variance will be judged against the corresponding increase in the amount of time spent processing a particle history.

\subsection{Organization of the Dissertation}

General background on the Monte Carlo method for solving radiation transport problems as well as the variance reduction technique of splitting/Russian roulette was presented in this chapter. Chapter II contains a review of some previous work by Everett and Cashwell which constituted the theoretical seed for this dissertation, as well as a semi-chronological overview of the developnent of the general theory of 
Monte Carlo errors. The analysis of splitting costs, which constitutes the most important aspect of this thesis, is based on the theory of errors. The characteristics of the transport model as well as the nature and scope of the analysis which was involved in this research are presented in Chapter III. The main theoretical development in formulating the moment equations for the Monte Carlo score and time per particle history (assuming a single splitting surface in the problem geometry) is contained in Chapter IV. The results of an extensive numerical study designed to find optimum spl* ing conditions for various values of transport model parameters are also presented. Chapter $V$ generalizes the analysis of Chapter IV to the case of multiple splitting surfaces and also contains some numerical results, including a comparison of splitting costs associated with Monte Carlo calculations employing optimal versus heuristically-prescribed values of splitting parameters. Finally, Chapter VI is reserved for conclusions and recommendations for future work. 
CHAPTER II

PREVI〜US STUDIES AND RELATED WORK

The material in Chapter II may still be regarded as introductory in nature. However, the information presented is quite specifically related to the original work comprising this dissertation. First, a summary of some previously reported analytical work by Everett and Cashwell is presented in order to introduce the basic features of splitting cost analysis. A discussion of these analytical results also allows for a clearer and more precise definition of the starting point for the original work of this dissertation. The second section of Chapter II provides a somewhat historical sketch of the development of the theory of Monte Carlo errors. The theoretical groundwork represented here forms the basis for the analysis used in this research to deterministically predict the computational cost associated with the implementation of a particular set of splitting parameters in a Monte Carlo calculation.

\subsection{Work of Everett and Cashwe11 on the Pure1y Absorbing Slab}

The first published results dealing with the quantification and optimization of the cost of splitting in Monte Carlo transport calculations (and consequently the theoretical seed for this research) appeared as a LASL (Los Alamos Scientific Laboratory) report authored by Everett and Cashwell.12 In this paper they presented a formal, analytical treatment of splitting applied to a fairly simple particle transport problem. 
The model they chose to analyze involved a one-dimensional semiinfinite slab with a thickness of D mean free paths (see Fig. 3). 'The medium was composed of a pure absorber $\left(\Sigma_{c}=\Sigma_{T}\right)$. Source neutrons were monodirectional and normally incident on the front surface of the slab $(z=0)$. The quantity to be estimated in the course of the Monte Carlo calculation was the expected particle transmission through the slab, i.e., leakage current at $z=D$. Splitting was assumed to occur in a ratio $\mathrm{m}: 1$ at a single, variably located, internal surface $z_{s}$, where $0<z_{s}<D$ [here $z_{s}$ and $D$ are in units of mean free paths (mfp)]. In this particular transport model, every collision made in the slab results in a termination of the particle or sub-particle by capture. The elimination of scattering facilitates the quantification of "success" and "failure" probabilities which characterize the particular stochastic experiment involved in this problem. There are (m +1$)$ possible outcomes of the experiment, each of which may be assigned a certain probability. For example, a 0 score for a history will result if either a particle does not surrive to the splitting surface, or (if it does survive and is split) all $\mathrm{m}$ sub-particles produced by the splitting do not survive to the tally surface. The appropriate score $[\mathrm{x}(0)]$ and probability $[\mathrm{p}(0)]$ associated with 0 particles reaching the tally surface may be formulated

$$
x(0)=0
$$

with

$$
p(0)=\left(1-e^{-Z s}\right)+e^{-Z s} Q^{m} .
$$




\section{Splitting At An Internal Surface, $z_{s}$}

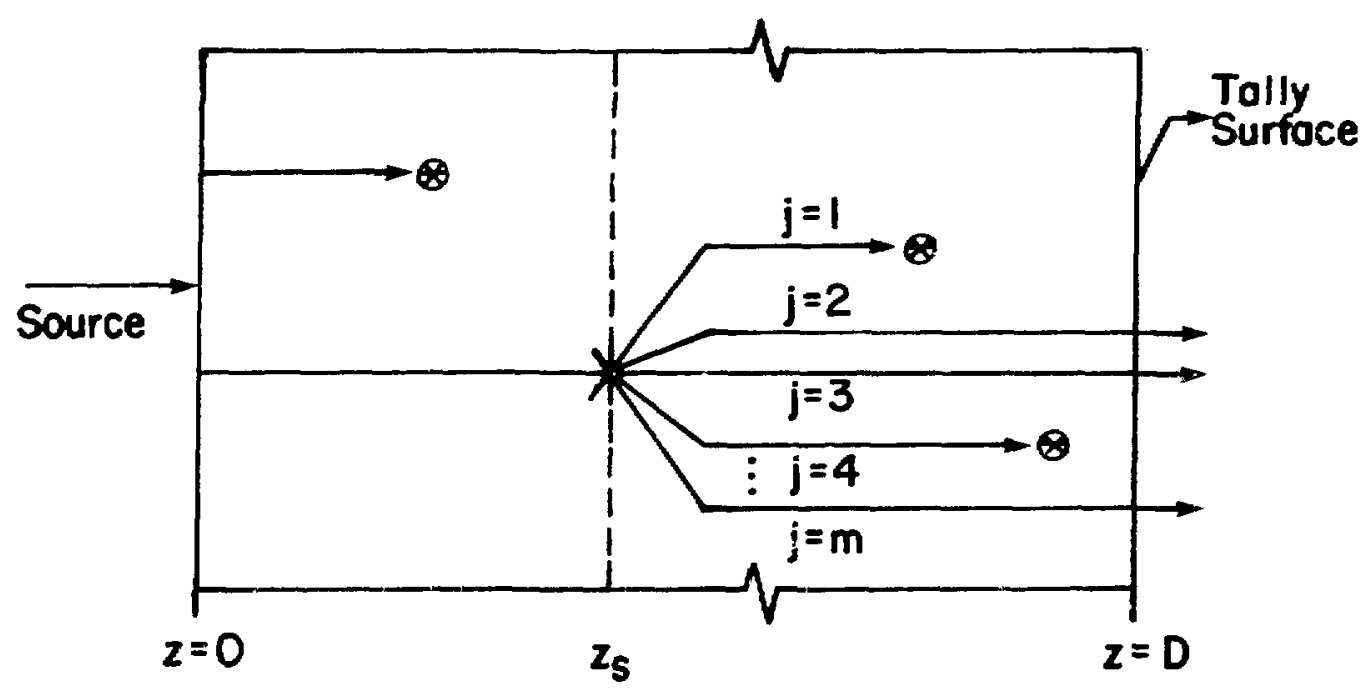

Figure 3. Transport Model Used as Basis for Everett and Cashwe11 Analysis.

(8 denotes analog capture.) 


$$
\begin{aligned}
Z \equiv \mathrm{e}^{-\left(\mathrm{D}-\mathrm{Z}_{\mathrm{S}}\right)}= & \begin{array}{l}
\text { probability of traversing }\left(\mathrm{D}-\mathrm{Z}_{\mathrm{s}}\right) \\
\text { without collision, i.e., "success"; } \\
\text { and }
\end{array} \\
Q \equiv 1-\mathrm{Q}= & \text { "fallure" probability. }
\end{aligned}
$$

Non-zero scores result if the particle first survives to the splitting surface where it is split into m sub-particles. Of these, only $i(i=1,2, \ldots, m)$ can survive the distance $\left(D-z_{s}\right)$. The possible outcomes (depending on i) are formulated

$$
x(i)=i / m
$$

with

$$
p(i)=e^{-Z} s\left(\begin{array}{c}
m \\
i
\end{array}\right) P^{i} Q^{m-i}
$$

In Eq. (2.1.2), ( $\left.\begin{array}{c}m \\ i\end{array}\right)$ represents the appropriate binomial coefficients. Thus by considering a binomial probability distribution in the description of the possible outcomes of this stochastic experiment, Everett and Cashwell were able to analytically express the first and second moments (hence, the expected sample variance) of the Monte Carlo score.

The time per particle history was also formulated analytically:

$$
\tau_{p}=\tau_{0}+e^{-Z} s\left(m \tau_{0}\right)=\tau_{0}\left(1+e^{-Z s} \cdot m\right)
$$

Here $\tau_{0}$ represents the elemental unit of computer time required in order to sample the distance to the first collision and compare this to the next surface. The factor $e^{-z}$ s represents the probability of survival to the splitting surface, and $\mathrm{m} \tau_{0}$ is the additional computer time required to track the (m) sub-particles generated at $\mathrm{Z}_{\mathrm{s}^{*}}$ 
Everett and Cashwell were then able to formulate the cost function for splitting at $\mathrm{Z}_{\mathrm{s}}$ :

$$
f_{c} \equiv \sigma_{s}^{2} \tau_{p}=\tau_{0} p_{t}^{2}\left\{\left[\frac{1}{m p_{t}}+(m-2)\right]+\left(\frac{m-1}{m}\right) e^{Z s}+\left(\frac{1}{p_{t}}-m\right) e^{-Z s}\right\} \text {, }
$$

where $\quad p_{t} \equiv e^{-D}$.

Further analysis revealed that for slabs of sufficient thickness, i.e., $D>\ln \left[\frac{m(m+3)}{m-1}\right]$, the cost function exhibits a minimum (optimum) value at $\mathrm{Z}_{0}$ where

$$
\mathrm{e}^{2 \mathrm{z}_{0}}=\left(\frac{\mathrm{m}}{\mathrm{m}-\mathrm{l}}\right)\left(\frac{1}{\mathrm{P}_{\mathrm{t}}}-\mathrm{m}\right) \quad ; \quad 0<\mathrm{z}_{0}<\mathrm{D}
$$

In this case, the splitting cost at $z_{0}$ is also less than the cost for an analog calculation, i.e., a no-splitting calculation.

A special-purpose Monte Carlo code was written in order to provide a stochastic model of the transport problem analyzed by Everett and Cashwe11. The intent was to obtain real Monte Carlo values of the cost function (subject to execution times on a CDC-7600 computer) as a verification of the analytical results. The coding was specifically designed so that the measured computer time per history would reflect as closely as possible only the timing for the operations considered in the analytical formulation of $\tau_{p}$ [see Eq. $\left.(2.1 .3)\right]$.

Figure 4 presents the analytical and stochastic results for the case involving $m=3$ and $D=3.0 \mathrm{mfp}$. Given the analog cost for this case was $\mathrm{f}_{0}=0.180 \mu \mathrm{sec}$, it can be seen that the minimum value of the 


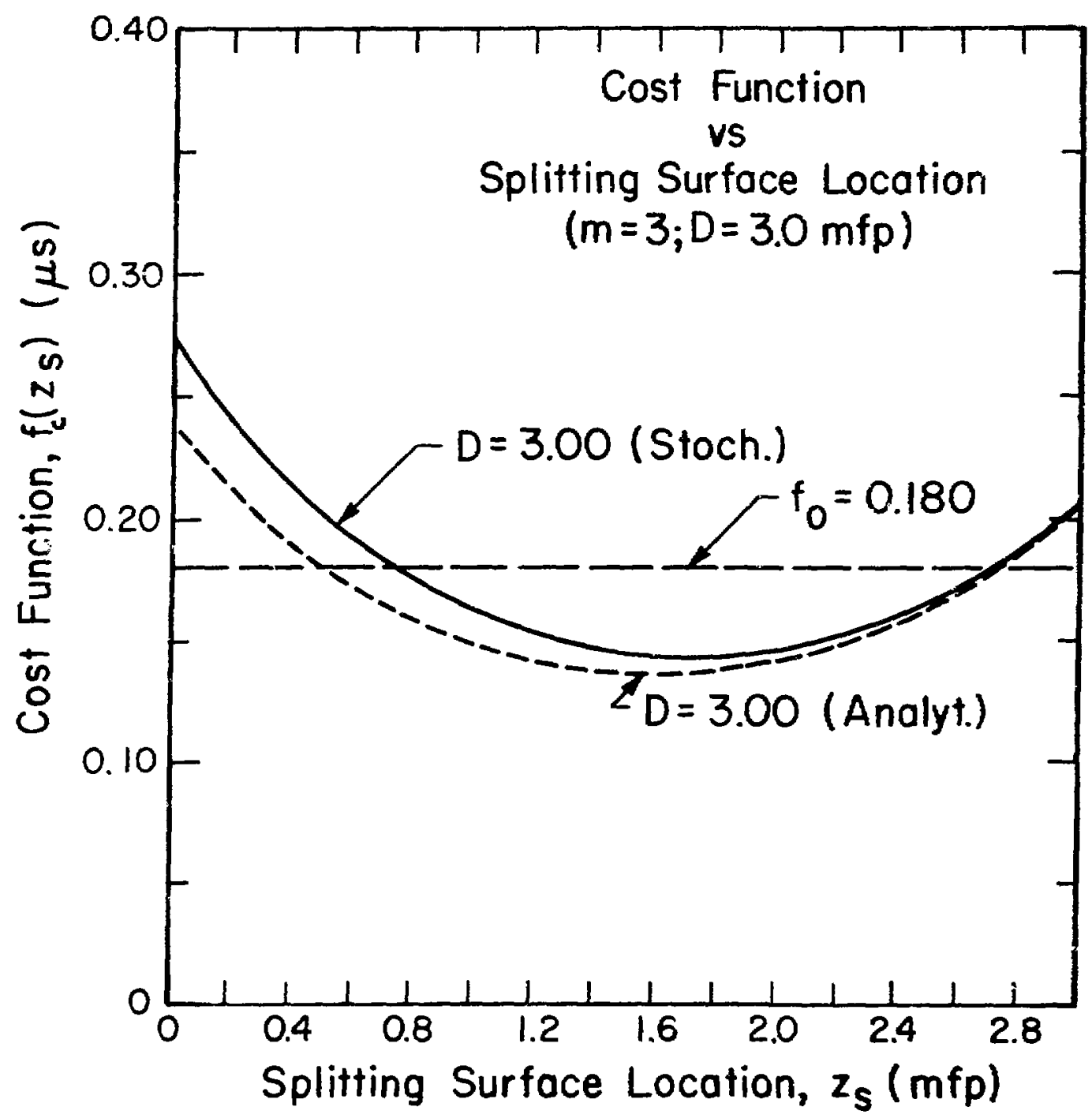

Figure 4. Verification of Everett and Cashwe11 Analysis: Cost Function vs. Splitting Surface Location. 
(analytic) cost function $\left[f_{c}\left(z_{0}\right)=0.136 \mu \mathrm{sec}\right.$ at $\left.z_{0}=1.6 \mathrm{mfp}\right]$ is lower than the analog cost. In fact, splitting is found to be cost-effective over a significant range of $\mathrm{z}_{\mathrm{s}}$. The discrepancy between the two curves in Fig. 4 results from the unavoidable increment in time due to extra coding (overhead) which must be included in order to perform the appropriate logic in processing the (m) sub-particles generated at the splitting surface. The (m - $\left.{ }^{0}{ }_{0}\right)$ considered by Everett and Cashwell is really a slight underestimate of the real computer time involved in processing the (m) sub-particles.

It is informative to consider the justification for the shape of the cost function curve, e.g., as exhitited in Fig. 4. The analytical behavior of the cost function actually derives from the relative effect arising from two competing tendencies (see Fig. 5). As $Z_{s}$ increases (splitting surface is located deeper into the slab and away from the source), the time per particle history decreases. Physically, as the splitting surface is located closer and closer to the tally surface, fewer source particles ever reach it. Thus, fewer particles are split, and the mean time to process a particle history decreases accordingly. On the other hand, the sample variance increases as the splitting surface is located closer to the tally surface (the bottom part of Fig. 5 shows the case for three different slab thicknesses). The limiting conditions are considered first. If the splitting surface were located at the tally surface $\left(Z_{s}=D\right)$, then no particle would ever be split effectively (in fact, every particle reaching the tally would be split and every single sub-particle thus produced would immediately score). There is then no essential difference relative to the analog 


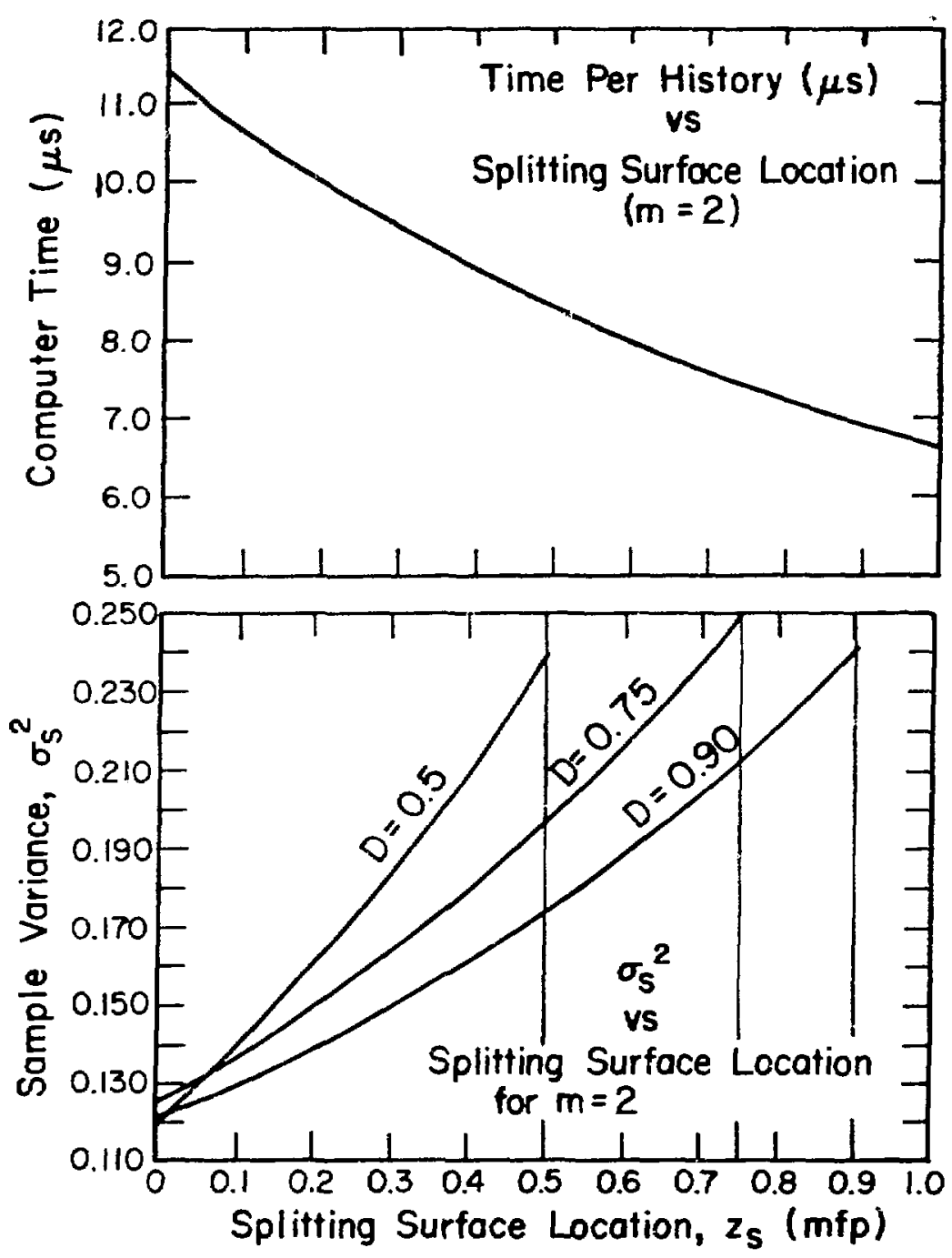

Figure 5. Behavior of $\tau_{p}$ (above) and $\sigma_{s}^{2}$ (below) with Splitting Surface Location. 
situation; $\sigma_{s}{ }^{2}$ in this case takes on the analog value, and variance reduction due to splitting is non-existent. In the other extreme, if the splitting surface is located at the source $\left(z_{S}=0\right)$, every single source particle is split. In this case, variance reduction is maximized; in fact, there is an m-fold reduction (c.f., Ref. 11, pp. 3-4). For intermediate $Z_{S}$, the sample variance increases monotonically. As the splitting surface is located deeper into the slab, fewer and fewer source particles are split. But more importantly, splitting becomes less and less effective. Given a small distance between splitting and tally surfaces, there is a greater chance that all (m) sub-particles generated at the splitting surface will score at the tally. As noted before, this gains one nothing over the anjog case, i.e., where the original source particle would contribute a score of 1 on its own. Splitting is only truly effective in reducing variance when, for a significant number of histories, several of the (m) sub-particles initiated at the splitting surface result in a tally, whereas others do not. Inly in this case is splitting productive in the sense that it gives a particle history $m(>1)$ chances to score (recall discussion of splitting in Chapter I). Thus as the splitting surface is located closer to the tally surface, the sample variance increases to the analog value.

The cost function $\mathrm{f}_{c}$, then, is itself a product of two functions-one monotonically increasing with $Z_{s}$, the secolnd monotonically decreasing. In certain cases (that is, for slab thicknesses as delimited by Everett and Cashwel1), the cost function may be minimized at a certain optimal value of $\mathrm{Z}_{\mathrm{s}}$ (see Fig. 4). However, this is not always the case. 
For thin slabs, the rise in $\sigma_{s}{ }^{2}$ with $z_{s}$ overwhelms the corresponding drop in $\tau_{p}$. As a result, in these cases the cost function is a monotonically increasing function of $Z_{s}$, everywhere greater in magnitude than the associated analog (no-splitting) cost. This behavior is exemplified in Fig. 6. In this case, the analog cost was $f_{0}=0.947 \mu \mathrm{sec}$.

To facilitate the analytical formulation of $\tau_{p}$, Everett and Cashwell considered only the time involved in executing the basic operations of the particle history [see Eq. (2.1.3)]. In a real calculation, however, some additional (essential) operations are required in processing a history. For example, in some more complicated problems, the time required to update the tallies and increment their śquares (for eventual computation of sample variance) after each particle history may be an important element of the overall time per history. Time is also involved in generating a source particle and incrementing tallies during the random walk. It was desired to see if including these extra operations in the time per history for the problem under consideration would or would not influence the cost-effectiveness of splitting. Accordingly, the Monte Carlo calculation used to verify the Everett and Cashwell analysis was redesigned with a logic more in accordance with standard production codes, e.g., MCNP. Timings were made to be more inclusive; i.e., $\tau_{p}$ reflected all computations during a particle history, more than just the basic operations considered by Everett and Cashwell. The results for various values of splitting ratio m and for $D=3.0 \mathrm{mfp}$ are displayed in Fig. 7.

Again, as in the previous calculations, the cost-effectiveness of splitting at an internal surface is assured. Due to the consideration 


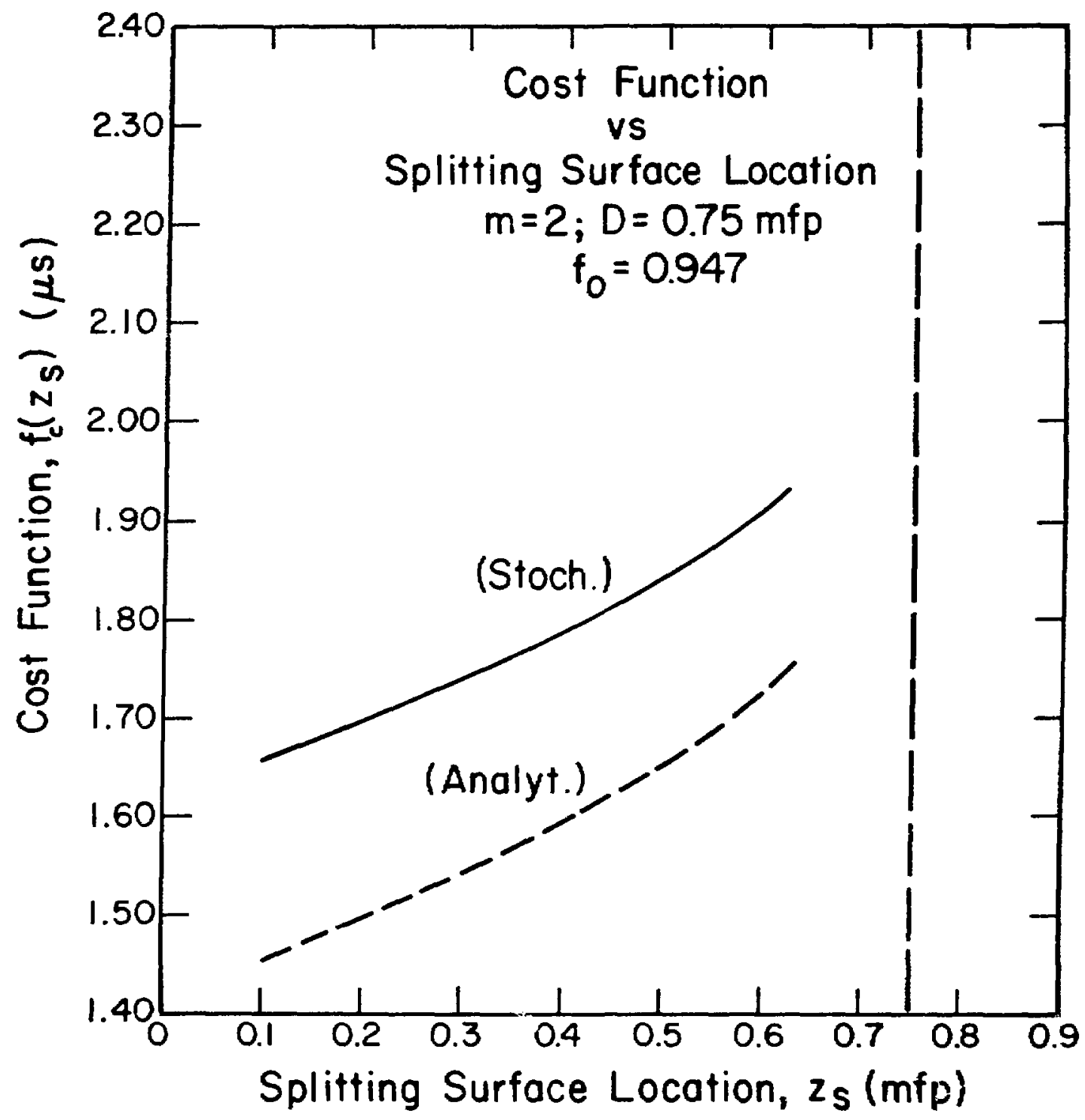

Figure 6. Example of Inefficient Splitting in Thin Slabs. 


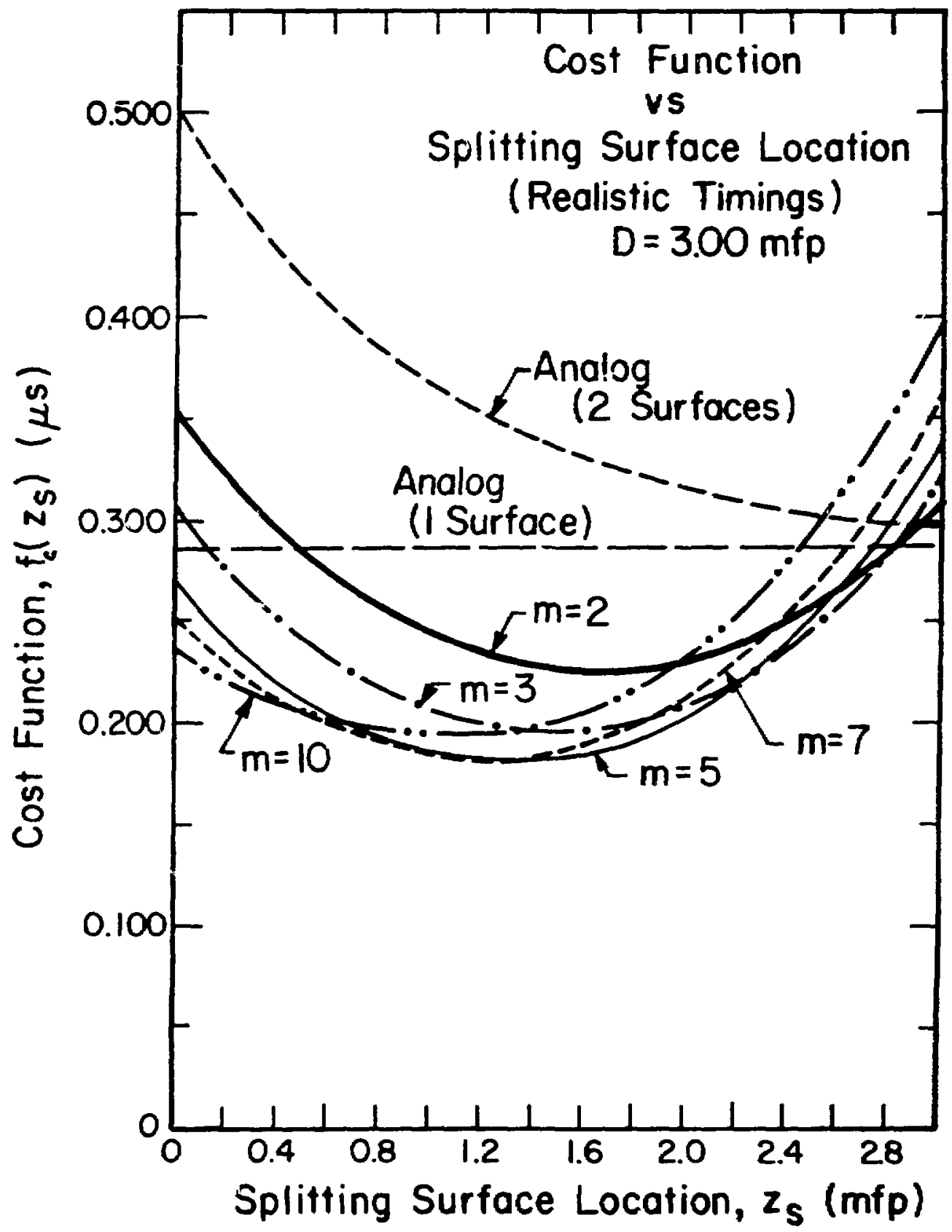

Figure 7. Cost Function vs. Splitting Surface Location $(\mathrm{D}=3.0 \mathrm{mfp} ; \mathrm{m}=2,3,5,7,10)$. 
of a greater number of operations in the tine per history, the magnitudes of the cost function were all scaled higher in this particular series of calculations. Nevertheless, the shape of the cost function was left unchanged, and the optimum splitting surface locations corresponding to minimum cost (for the different $m$ values) were within $0.1 \mathrm{mfp}$ of the optimum locations predicted by Everett and Cashwell. Figure 7 displays two curves for the analog cost. The "analog cost ( 1 surface)" refers to the case where no additional internal surface is present. After the particle leaves the source, it can encounter only one more surface, the tally surface. On the other hand, "analog (2 surfaces)" in the figure refers to the case where a non-splitting internal surface is geometrically defined. The importances on either side of this surface, however, are equal--hence, no splitting takes place. But on leaving the source, the particle now may encounter two surfaces (internal and tally), and the corresponding penalty in computer time is reflected in the appropriate curve displayed in Fig. 7. In summary then, the analysis presented by Everett and Cashwe11 based on a transport model involving a purely-absorbing medium in onedimensional slab geometry allows for the prediction of optimum splitting surface location and splitting ratio (given a fixed slab thickness). Under the right conditior 3 , the value of the cost function attending splitting was found to be lower than the corresponding cost of an analog computation over a relatively wide range of $Z_{s}$.

The goal of this dissertation is to extend the analysis of splitting costs to a more complicated and realistic transport problem-specifically, one that would include the process of particle scattering. 
The consideration of scattering precludes the straightforward application of the binomial probability distribution and makes the a priori analysis related to the expected sample variance and computer time per particle history a great deal less tractable. Nevertheless, as will be seen in the ensuing section, the theory of Monte Carlo errors provides an appropriate analytical tool with which (upon the implementation of certain modifications and extensions) the splitting cost analysis may be applied to a more complicated particle transport model.

\subsection{Analysis of Error in Monte Carlo Calculations}

In this section, the development of a theoretical basis for predicting Monte Carlo errors will be highlighted in a somewhat chronological fashion. The material summarized here is especially significant in that it provides the theoretical foundation upon which the analysis and results of this dissertation are based. Specifically, this dissertation is an example of how the generalized theory of Monte Carlo errors may be employed in the analysis and optimization of an important variance reduction technique, $i . e$. , in the solution of a practical problem which derives from the general objestive of ensuring efficiency in Monte Carlo calculations.;

The earliest work to be cited here is that of Coveyou, Cain, and Yost. 13 The significance of this work lies in the fact that it underscores the central importance of the adjoint formulation of the particle transport problem to the analysis of variance reduction in Monte Carlo calculations. Specifically, the authors introduce a value function which is used to facilitate the analytical formulation of expected 
values and the variance of Monte Carlo estimates. The value function $W(\underline{R})$ is the expected total contribution (present and future, but not past) of a particle to the Monte Carlo tally given the particle is coming into a collision event at phase-space point $\underline{R}$. Mathematically, the value function is the solution of the integral equation of the adjoint formulation to the particle transport problem. The authors derive a generailized analytical representation for the expected sample variance in terms of this value function. The formalism is extended to such standard variance reduction techniques as source biasing, survival biasing, and density biasing (importance sampling). Unforcunately, the solution to the value equation is just as difficult as the direct solution of the original particle transport problem, and thus approximations to the value function must usually be employed in applications of the theory. Nevertheless, the authors elucidated a fundamental connection between variance reduction analysis and adjoint solutions of the transport problem.

Amster and Djomehri ${ }^{14}$ have shown that the determination of expected variance may result from the successive solution to two coupled integral equations. They based their study on an analog Monte Carlo game. In the most general case, each collision a particle makes (a non-multiplying medium is assumed) may result in a score which has a known probability distribution. The total tally score for the particle history is the sum of all collision scores over that particular history. The authors introduce the history score accumulation probability $\Psi(\underline{R}, s)$ ds which is the probability that a particle introduced into the system at $\underline{R}$ will eventually contribute a tally score $s$ in ds over its history. 
They proceed to derive equations for the $r-t h_{1}$ moment of the score about the distribution function

$$
M_{r}(\underline{R})=\int_{-\infty}^{+\infty} s^{r} \psi(\underline{R}, s) d s .
$$

The appropriate integral equations for $r=1$ and 2 (first and second moments) are thus derived. The recursive solution of these two coupled integral equations allows for the prediction of the Monte Carlo sample variance. It can also be shown that the integro-differential form of the $r$-th moment equation (which can be derived from the integral form) is itself adjoint to the corresponding form of the Boltzmann transport equation. This suggests that standard numerical techiiques may possibly be employed in the solution for the first and second moments. Recall that the work of Amster and Djomehri considered scoring to take place at collision sites. The theory was extended by Booth and Amster ${ }^{15}$ to include track-Iength estimators (used, for example, in the estimation of flux in a cell by tallying the particle's track length per unit volume of that cel1). The appropriate generalization was made which allowed the score distribution functions at individual events to depend on the phase-space coordinates corresponding to two successive collisions.

Finally, Booth ${ }^{16}$ and Booth and Cashwel1 $1^{17}$ have generalized the theory of errors as introduced by Amster and Djomehri to account for nonanalog Monte Carlo simulations. Also, multiplying systems (even supercritical) as well as time-dependence were considered in their 
formulations. The work is particularly noteworthy from the standpoint of this dissertation, since the authors incorporated the treatment (albeit a formal, generalized one) of splitting and Russian roulette in the theory. In addition to allowing for the prediction of the variance of conventional Monte Carlo tallies, the integral equations for the r-th moment of the history score as presented by Booth and Cashwell also facilitate the prediction of time per particle history. If one defines the score at each event to be the actual computer time required to process that event, then the appropriate integral moment equation may be employed to express the expected value (first moment) of the time per particle history.

Independently of the work of Booth and Cashwell, Sarkar and Prasad 18 have also generalized the theory of errors to treat nonanalog games. A special emphasis was placed on the application of the theory in the optimization of the exponential transformation. Although splitting and Russian roulette are considered in their work, the techniques are implemented in conjunction with the exponential transformation and are treated in a nonstandard manner--they are performed as particles leave collision sites, with the objective of keeping the particle weight close to unity.

Finally, Lux ${ }^{19}$ has also employed a generalized theory of errors in developing sufficient conditions under which variance may be reduced when considering survival biasing schemes.

All of the papers cited above provide valuable insight into the problem of Monte Carlo error prediction. However, the work of Booth 16 
was most instrumental in providing the theoretival basis for the analysis fundamental to this present work, i.e., splitting cost analysis and optimization. 
CHAPTER III

NATURE AND SCOPE OF PROBLEM TO BE STUDIED

The aim of this chapter is to introduce in greater detail the specific problem to be considered in this work. First, the characteristics of the particle transport model are presented. This particular model will be used as a basis for the analysis to be presented in this dissertation. The model also facilitates the definition of the scope and limitations of this work. The latter part of the chapter serves to motivate the analysis which will be developed in subsequent chapters.

\subsection{Defining the Characteristics of the Transport Mode1}

As was discussed in Chapter II, the first formal study relating to splitting costs in Monte Carlo transport calculations was presented by Everett and Cashwe11. 12 By considering a purely-absorbing medium they were able to apply an exact analytical treatment to the problem. The work presented in this dissertation extends the analysis of splitting costs to a more complicated and realistic transport model--specifically, one that includes the possibility of particle scattering.

The transport model under study here is similar to the one analyzed by Everett and Cashwe11. Again, a one-dimensional, semi-infinite slab geometry is considered (the slab is $D$ mean free paths in thickness). Particles are considered to be normally incident on the front surface of the slab $(z=0)$. The Monte Carlo estimate of interest (tally) is the probability of transmission through the slab (in other words, the expected leakage current at the tally surface, $z=D$ ). 
However, several additional and complicating features are included in the present model:

- macroscopic scattering cross section is nonzero, i.e., $\Sigma_{T}=\Sigma_{C}+\Sigma_{S}$,

- scattering is assumed isotropic in the laboratory reference frame,

- a single energy-group treatment is considered,

- survival biasing (that is, the weighting-out of absorptions) is considered in all cases, and

- splitting (m:1) and Russian roulette are invoked at one or more internal surfaces $\left(z=z_{s, 1}, z=z_{s, 2}\right.$, etc.); only integer splitting ratios are considered, i.e., $\mathrm{m}=2,3,4$, etc.

A graphical depiction of the model is presented in Fig. 8 .

\subsection{Nature of the Analysis to be Applied to the Problem}

The complications to the transport model noted above preclude a simple, straightforward analysis. It is no longer possible to apply directly a binomial probability distribution in accounting for the possible outcomes of the stochastic experiment. Indeed, the probabilities of "success" and "failure" are no longer easily defined (except in a very formal manner). One need only consider the Neumann Series expression for the collision density to realize that the particles contributing to the tally may correspond to histories which up to that point could have had any finite number of previous collisions, with a corresponding distribution in contributing weights. As implied in section 3.1 , scattering also allows for the explicit treatment of Russian roulette upon backscatter into regions of Iower importance. Nevertheless, in 


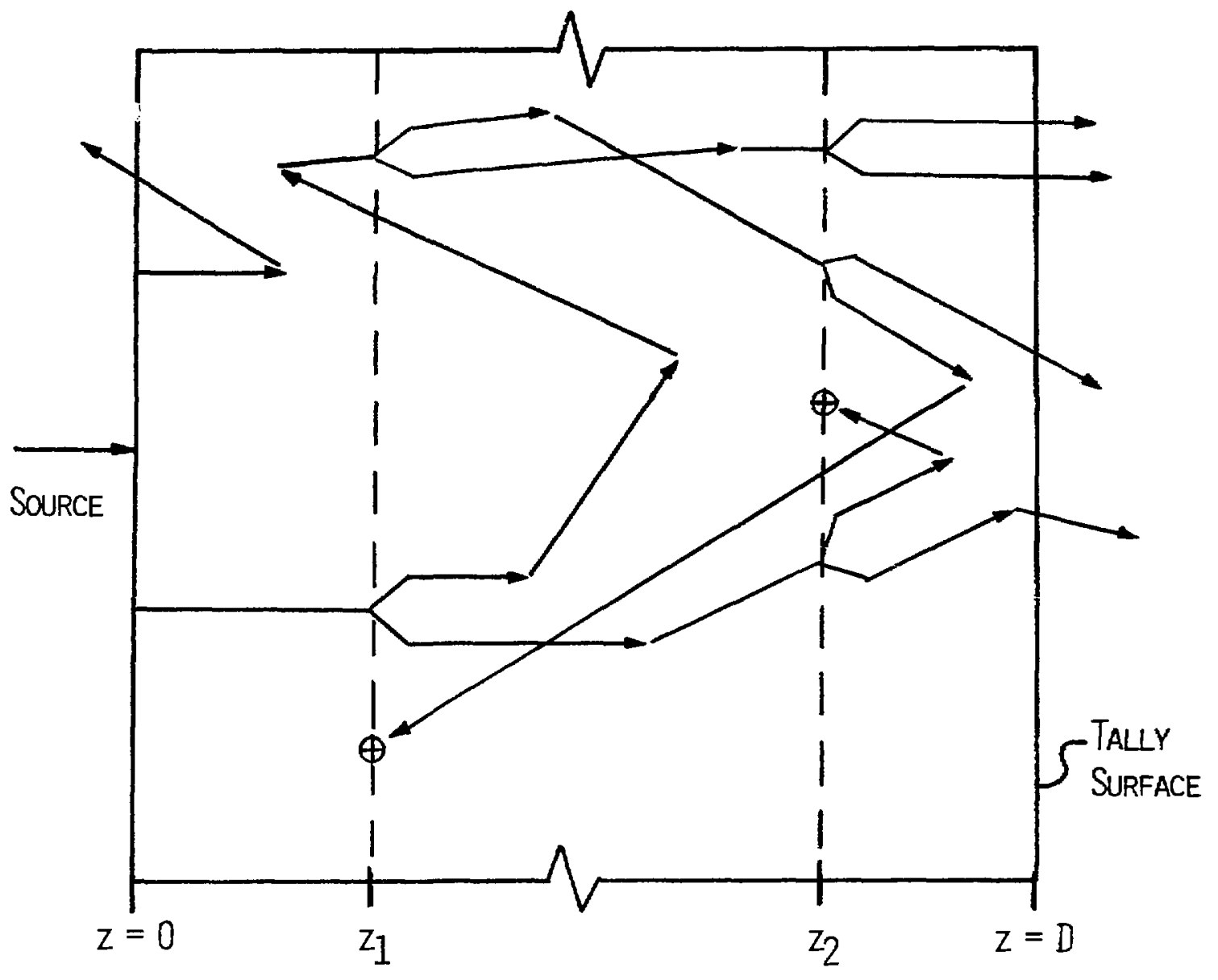

Figure 8. Transport Model Used as Basis for Analysis (Isotropic Scattering Included).

[ $\oplus$ denotes termination by Russian roulette.] 
order to predict the value of the cost function associated with a given set of splitting parameters, it is necessary to formulate a priori its two components: the expected (true) sample variance of the Monte Carlo score $\left(\sigma_{s}{ }^{2}\right)$ and the expected time required to process a particle history $\left(\tau_{p}\right)$. To quote Amster and Djomehri: 14

"The problem posed by the error calculation is approximateiy as difficult as the Monte Carlo problem considered."

As noted in Chapter II, a generalized theoretical basis for predicting Monte Carlo errors has been developed. In particular, the specific generalizations of Booth ${ }^{16}$ and Booth and Cashwel1 17 to formally include splitting and Russian roulette in the theory make it quite applicable as an analytical tool in treating the central problem of this dissertation. With certain modifications and the appropriate specification of probability distributions, the theory may be applied to the Monte Cario transport problem presented in section 3.1. Thus, the appropriate integral equations governing the first and second moments of the slab transmission score, as vell as the first moment (expected value) of time per particle history, were developed. [Splitting at an internal surface (rather than formally splitting from a distribution in phase space) required special treatment in the formulations for the second moment of the score and $\tau_{p}$, as will be detailed in Chapter IV.]

The integral moment equations were then converted to an integrodifferential form. In this form, the equations represented adjoint formulations of the one-speed Boltzmann transport. equation. This 
immediately suggested a numerical solution technique employing a standard discrete ordinates $\left(\mathrm{S}_{\mathrm{n}}\right)$ transport code. Thus, the numerical solution for $\sigma_{\mathrm{s}}{ }^{2} \tau_{\mathrm{p}}$ [actually, separate solutions for the first and second moments of the transmission $\left(\langle x\rangle,\left\langle x^{2}\right\rangle\right)$, as well as $\left.\tau_{p}\right]$ allowed for the prediction of cost function behavior under a wide variety of splitting conditions.

The significance of the present work to Monte Carlo methodology is underscored by several considerations. First, the technique of splitting as commonly implemented in stardard Monte Carlo codes has never undergone an adequate quantitative analysis (other than the work of Everett and Cashwe11). The research presented here hopefully represents a positive step in this direction. The present work also provides an example of how the generalized theory of Monte Carlo errors may be applied to a practical problem involving the effective implementation of a standard variance reduction device in a radiation transport calculation. Although the transport model considered in this particular analysis may seem fairly uncomplicated, it nevertheless represents fairly well the condition under which geomet. ic splitting is apt to be extensively applied, namely in radiation transport problems involving deep penetration. In such Monte Carlo calculations, one of the prime considerations is where to place splitting surfaces along the straightline mean-free-path distance separating the source and exit surfaces. Finally, the deterministic calculation of the cost associated with a given set of splitting parameters facilitates an a prior 1 optimization of the technique. Moreover, it provides a quantitative analytical basis 
for judging the relative merits of various rules of thumb which are commonly erployed by users in implementing splitting in a Monte Carlo calculation. It is not presumptious to state that such an analysis is intellectually more satisfying than a complete rellance on experience or insight. 
CHAPTER IV

THE CASE OF A SINGLE SPLITTING SURFACE

The material in this chapter is devoted to the analysis of a single internal splitting surface, variably located in tne slab at $z=z_{s}\left(0<z_{s}<D\right)$. The theory of Monte Carlo errors is applied to the specific transport model being considered here. Integral equations for the first and second moments of the slab transmission score $(\langle x\rangle$ snd $\left\langle x^{2}\right\rangle$, respectively) and the expected value of time per particle history $\left(\tau_{p}\right)$ will be derived by closely following the derivation of the generalized $r-t h$ moment equations for the Monte Carlo score as demonstrated by Booth. 16 However, in all cases only specific features pertinent to the transport model as defined in Chapter III will be included in the derivations presented here. The applicable probability distributions will be appropriately specified and several features important to this development but not treated explicitly in the Booth formulations will be given special attention. Booth's generalized time-dependent equations do not explicitly reflect the escape of particles past the outer boundaries of a defined geonetry. However, this is essential to the problem being considered her $\geqq$. The analytical treatment in this case, nevertheless, parallels very closely the treatment given in the general theory for particles experiencing a collisionless free-flight for the duration of time being considered. In effect, an escape from the defined geometry is tantamount to a collisionless free-flight for infinite time (in the context of the time-dependent generalized theory). Closely related to this, the general theory does not consider the intersection of a 
particle's flight with a fixed surface in the geometry, e.g., an internal splitting surface. In the Booth formulations, splitting and Russian roulette events are formally sampled from generalized probability distributions defined on phase space. For the problem being considered here, splitting at an internal surface requires special treatment in formulating the integral equations. This is ultimately reflected in the discontinuous behavior of $\left\langle x^{2}\right\rangle$ and $\tau_{p}$ at the splitting surface.

Following the derivation of the integral moment equations, their conversion to an integro-differential form is discussed. A solution technique employing $S_{n}$ calculations is then described, and the numerical results of a parameter study are presented.

\subsection{Deterministic Prediction of Sample Variance}

In this section, the equations for the first and second moments of the slab transmission score are developed. Solution for the first two moments $\left(\langle x\rangle\right.$ and $\left\langle x^{2}\right\rangle$ ) then allows for the deterministic prediction of the sample variance $\left(\sigma_{s}^{2}=\left\langle x^{2}\right\rangle-\langle x\rangle^{2}\right)$.

A lengthy list of definitions precedes the derivation itself. Implicit in all these definitions is the fact that for the monoenergetic transport problem in one-dimensional semi-infinite slab geometry, generalized phase-space coordinates reduce to two variables. These are the particle's position in the slab measured along the normal direction (z) and its direction of travel relative to the normal (the direction is expressed in terms of $\mu$, the cosine of the angle between the direction of travel and the normal direction). In order to facilitate a clearer exposition of the logic involved in the development, the derivations are 
motivated and begun in terms of generalized distribution functions and phase-space coordinates. At an appropriate point in the development, substitution is made in order to present the equations only in terms of the specific variables which are characteristic of the transport model as defined in Chapter III.

\subsubsection{Derivation of the Integral $r$-th Moment Equations $(r=1,2)$ for the Slab Transmission Score.}

The following definitions are important to the derivations which are presented in this dissertation:
$\mathbf{z}$
$\equiv$ Particle position coordinate in semi-infinite slab geometry.
$\mu$
$\equiv$ Particle directional coordinate (cosine of the angle between the direction of travel and the normal direc- tion).
$\underline{\mathbf{R}}$
$\equiv$ Phase-space vector for the time-independent, mono- energetic transport problem in semi-infinite slab geometry.
$=(z, \mu)$.
$\underline{\mathbf{P}}$
$\equiv$ Augmented phase-space vector (includes particle weight),
$=(z, \mu, w)$, where $w=$ particle weight.
$\mathbf{s}$
ミScore that a particle makes (in the general case, a particle might score at any of the possible events it may encounter).
$\left.\begin{array}{l}\mathrm{p}_{E, 0}\left(\underline{P}^{\prime}, s\right) d s \\ \mathrm{P}_{E, D^{\prime}}\left(\underline{P}^{\prime}, s\right) d s \\ \mathrm{p}_{S}\left(\underline{P}^{\prime}, s^{\prime}\right) d s \\ \mathrm{P}_{S m}\left(\underline{P}^{\prime}, s\right) d s \\ P_{R}\left(\underline{P}^{\prime}, s\right) d s\end{array}\right\}$
$\equiv$ Score distribution functions, expressing the prob- ability that a particle will contribute a score in ds about $s$ given that the event at $\underline{p}^{\prime}$ is (respectively): an escape past the front surface $\left(z^{-}=0\right)$, an escape past the back surface $\left(z^{\prime}=D\right)$, a scattering event, an m:1 splitting event, or a Russian roulette event.
$\Sigma_{\mathrm{S}}, \Sigma_{\mathrm{T}} \quad \equiv$ Macroscopic scattering and total cross sections. 


$$
\begin{aligned}
& T\left(\underline{P}, \underline{P}^{+}\right) \mathrm{d} \mu^{+} \mathrm{dw}^{+} \quad \equiv \text { Transaission (free-flight)probability. This is the } \\
& =T\left(z \rightarrow z^{+}, \mu\right) \delta\left(\mu^{+}-\mu\right) \delta\left(w^{+}-w\right) d \mu^{+} d_{w^{+}}, \\
& \text {where } T\left(z \rightarrow z^{+}, \mu\right)=\left\{\begin{array}{l}
\exp \left(-\Sigma_{T}\left[\frac{z^{+}-z}{\mu}\right]\right) \text { for }\left[\frac{z^{+}-z}{\mu}\right] \geq 0, \\
0 \text { for }\left[\frac{z^{+}-z}{\mu}\right]<0 .
\end{array}\right.
\end{aligned}
$$

Note: The delta functions in the definition above reflect the fact that no directional or weight changes take place along the Eree-flight.

$$
\begin{aligned}
& \sigma\left({\underline{P^{+}}}^{+}, \underline{\mathrm{P}}^{\prime}\right) \mathrm{dz}^{+} \mathrm{dP}^{-} \equiv \text { Probability that a collision will take place in } \mathrm{dz}^{+} \\
& =\frac{\Sigma_{T}}{|\mu|} \delta\left(\underline{R}^{\prime}-\underline{R}^{+}\right) \delta\left(w^{-}-w^{+}\right) d z^{+} d \underline{P}^{-} .
\end{aligned}
$$

In the general case, a weight change may possibly take place due to biasing of the total cross section. However, as is indicated by the delta function, in this particular problem no such change occurs. [Note: $\mathrm{P}^{-}$ does not represent the phase-space coordinates as the particle leaves the collision; see definition of exit kerne1 $\left.\mathrm{E}\left(\underline{\underline{P}^{\prime}, \underline{P}^{\prime \prime}}\right).\right]$

$$
\begin{aligned}
& \mathrm{w}_{\mathrm{S}} \quad \begin{aligned}
\text { Weight multiplier used to reduce particle weight to } \\
\text { account for absorption at a collision, }
\end{aligned} \\
& =\Sigma_{\mathrm{S}} / \Sigma_{\mathrm{T}} \quad \text { (non-absorption probability). } \\
& . \mathrm{S}_{\mathrm{m}}\left(\mathrm{z}, \underline{\mathrm{P}}^{+}\right) \mathrm{dz} \mathrm{z}^{+} \equiv \begin{array}{l}
\text { Probability of experiencing an m:I splitting event in } \\
\mathrm{dz} \mathrm{t}^{+} \text {about } \mathrm{z}^{+}, \text {given the particle starts its current } \\
\text { free flight at } \mathrm{z} \text { and reaches } \mathrm{z}^{+} \text {without collision, }
\end{array} \\
& =
\end{aligned}
$$


where $\mathrm{H}\left(\mu^{+}\right)$is the Heaviside function:

$$
\mathrm{H}\left(\mu^{+}\right) \equiv \begin{array}{lll}
1 & \text { for } \mu^{+} \geq 0 \\
0 & \text { for } \mu^{+}<0
\end{array} .
$$

Thus, a splitting event will cccur with certainty at $z^{+}=z_{s}$, only if the particle crosses the splitting surface from the left (refer to Fig. 8).

$\mathrm{R}_{\mathrm{m}}\left(z, \underline{\mathrm{P}}^{+}\right) \mathrm{d} \mathrm{z}^{+} \quad \equiv$ Probability of experiencing a Russian roulette event in $\mathrm{dz}^{+}$about $\mathrm{z}^{+}$, given the particle starts its current flight from $z$ and reaches $z^{+}$without collision,

$$
=\left\{\begin{array}{c}
0 \quad \text { for } \quad z \leq z_{S}, \\
R_{m b}\left(L^{+}, \mu^{+}, w^{+}\right) d z^{+}=\left[1-H\left(\mu^{+}\right)\right] \delta\left(z^{+}-z_{S}\right) d z^{+} \\
\text {for } z>z_{S} .
\end{array}\right.
$$

$\mathrm{D}_{0}\left(\underline{\mathrm{P}}, \underline{\mathrm{P}}^{+}\right) \mathrm{d}^{+} \quad \equiv$ Probability of escaping the front surface at $\mathrm{dP}^{+}$about $\underline{\mathrm{P}}^{+}$, given that the current flight of the particle started at $\underline{P}$,

$$
=\left\{\begin{array}{r}
T\left(z \rightarrow z^{+}, \mu\right)[1-H(\mu)] \delta\left(\mu^{+}-\mu\right) \delta\left(w^{+}-w\right) \delta\left(z^{+}\right) d z^{+} d \mu^{+} d w^{+} \\
\text {for } z \leq z_{s}, \\
0 \quad \text { for } z>z_{s} .
\end{array}\right.
$$

Note: A front-surface escape is impossible as a nextevent if the particle starts out to the right of the splitting surface.

$\mathrm{D}_{\mathrm{T}}\left(\underline{\mathrm{P}}, \underline{\mathrm{P}}^{+}\right) \mathrm{d \textrm {P } ^ { + }} \quad \equiv$ Probability of escaping the back surface at $\mathrm{dP}^{+}$about $\underline{\mathrm{P}}^{+}$, given that the current flight originated $\bar{a} \mathrm{t} \underline{\mathrm{P}}$,

$$
=\left\{\begin{array}{r}
T\left(z \rightarrow z^{+}, \mu\right) H(\mu) \delta\left(\mu^{+}-\mu\right) \delta\left(w^{+}-w\right) \delta\left(z^{+}-D\right) d z^{+} d \mu^{+} d w^{+} \\
\text {for } z \geq z_{s} \\
\text { for } z<z_{s} .
\end{array}\right.
$$




$$
\begin{aligned}
& \mathrm{E}\left(\underline{\mathrm{P}}^{\prime}, \underline{\mathrm{P}}^{\prime}\right) \mathrm{d} \underline{\mathrm{P}}^{\prime} \quad \text { Probability that a collision at } \underline{\mathrm{P}}^{-} \text {will result in a } \\
& \text { change of phase-space coordinates to } \underline{\mathrm{P}}^{\prime \prime} \text { about } \underline{\mathrm{P}}^{\prime} \text {, } \\
& =E\left(z^{\prime}, \mu^{\prime} \rightarrow \mu^{\prime \prime}\right) \delta\left(z^{\prime}-z^{\prime}\right) \delta\left(w^{\prime}-w_{\mathrm{s}^{\prime}} \mathrm{w}^{\prime}\right) \mathrm{d} z^{\prime \prime} \mathrm{d} \mu^{\prime} \mathrm{dw}^{\prime \prime}, \\
& \begin{array}{l}
\text { where } E\left(z^{\prime}, \mu^{\prime} \rightarrow \mu^{\prime}\right) \mathrm{d} \mu^{\prime \prime}=\frac{\mathrm{d} \mu^{\prime \prime}}{2} \text { for isotropic scatter- } \\
\text { ing. }
\end{array} \\
& \text { Since absorptions are being weighted-out, every } \\
& \text { collision is treated as a scattering event, and the } \\
& \text { particle weig'lt is reduced by the factor } w_{s} \text {. } \\
& \mathrm{B}_{\mathrm{Sm}}\left(\underline{\mathrm{P}}^{+}, \underline{\mathrm{P}}^{\prime \prime}\right) \mathrm{d} \underline{\mathrm{P}}^{\prime} \quad \equiv \text { Probability that all }(\mathrm{m}) \text { particles resulting from a } \\
& \text { splitting event at } \underline{\mathrm{P}}^{+} \text {will each exit in } \mathrm{dP}^{-} \text {about } \\
& \underline{\mathrm{P}}^{\prime} \text {; in effect, only a weight change results, } \\
& =\delta\left(z^{\prime}-z^{+}\right) \delta\left(\mu^{\prime}-\mu^{+}\right) \delta\left(w^{\prime}-w^{+} / m\right) d z^{\prime} d \mu^{\prime} \mathrm{d}^{\prime} \text {. } \\
& \xi_{\mathrm{m}} \quad \equiv \text { Russian roulette survival probabilicy } \\
& =1 / \mathrm{m} \\
& =\delta\left(z^{-}-z^{+}\right) \delta\left(\mu^{-}-\mu^{+}\right)\left\{\xi_{m} \delta\left(w^{-}-w^{+} / \xi_{m}\right)\right. \\
& \left.+\left(1-\xi_{\mathrm{m}}\right) \delta\left(\mathrm{w}^{-\mu}\right)\right\} \mathrm{dz}{ }^{-} \mathrm{d} \mu{ }^{-} \mathrm{d} \mathrm{w}{ }^{\prime}, \\
& =\beta_{0 m}\left(\underline{P}^{+}, \underline{\mathrm{P}}^{-}\right) \mathrm{d} \underline{\mathrm{P}}^{\prime-}+\left(1-\xi_{\mathrm{m}}\right) \delta\left(z^{-}-\mathrm{z}^{+}\right) \delta\left(\mu^{-}-\mu^{+}\right) \\
& \times \delta\left(\mathrm{w}^{\prime \prime}\right) \mathrm{d} z^{\prime} \mathrm{d} \mu^{\prime} \mathrm{d} \mathrm{w}^{\prime \prime} \text {. } \\
& \beta_{0 \mathrm{~m}}\left(\underline{P}^{+}, \underline{P}^{-}\right) \mathrm{dP}^{-\infty} \quad \equiv \begin{array}{l}
\text { Russian roulette survival coordinate-change } \\
\text { probability, }
\end{array} \\
& =\xi_{\mathrm{m}} \delta\left(z^{\prime}-z^{+}\right) \delta\left(\mu^{\prime}-\mu^{+}\right) \delta\left(w^{\prime}-w^{+} / \xi_{m}\right) \mathrm{d} z^{\prime} d \mu^{\prime} \mathrm{dw}^{\prime} .
\end{aligned}
$$


Based on the foregoing definitions, the first task at hand is to formulate the score accumulation probability, that is

$$
\begin{aligned}
\psi(\underline{\mathrm{P}}, \mathrm{s}) \mathrm{ds} \equiv & \text { probability that a particle introduced into the } \\
& \text { system at } \mathrm{P} \text { (or continuing from an event at } \mathrm{p}) \\
& \text { will contribute during its remaining history a } \\
& \text { total score } \mathrm{s} \text { in ds. }
\end{aligned}
$$

Once a particle is introduced at $\underline{\mathrm{P}}$, it will begin a free flight, which can terminate in a number of possible "next events." As stated before, however, the imposition of an internal splitting surface ccmplicates the formulation somewhat in that the list of possible next events may differ, depending on where (relative to the splitting surface) the particle begins its flight. Accordingly, $\psi$ is formulated for three cases, depending on whether the particle starts its flight (at $z$ ) to the left of the splitting surface, to the right of the surface, or on the splitting surface:

$$
\begin{aligned}
& \psi_{1}(\underline{P}, s) d s \text { for } 0 \leq z<z_{s} \text {, } \\
& \Psi_{2}(\underline{P}, s) d s \text { for } z_{s}<z \leq D \text {, and } \\
& \psi_{z_{s}}(\underline{P}, s) d s \text { for } z=z_{s}
\end{aligned}
$$

We consider first the case of a particle introduced at $\underline{P}$, such that the starting location $z$ is to the left of the splitting surface. The flight from $\underline{P}$ may terminate at $\underline{P}^{+}$with any of three possible next events: a scattering collision at $\underline{\mathrm{P}}^{+}$(with $\mathrm{z}^{+}$such that $0<\mathrm{z}^{+}<\mathrm{z}_{\mathrm{S}}$ ), an escape past the front surface $\left(\underline{p}^{+}\right.$with $\left.z^{+}=0\right)$, or a splitting event ( $\underline{P}^{+}$with $\left.z^{+}=z_{s}\right)$. A graphical depiction of the possibilities is presented in Fig. 9. 


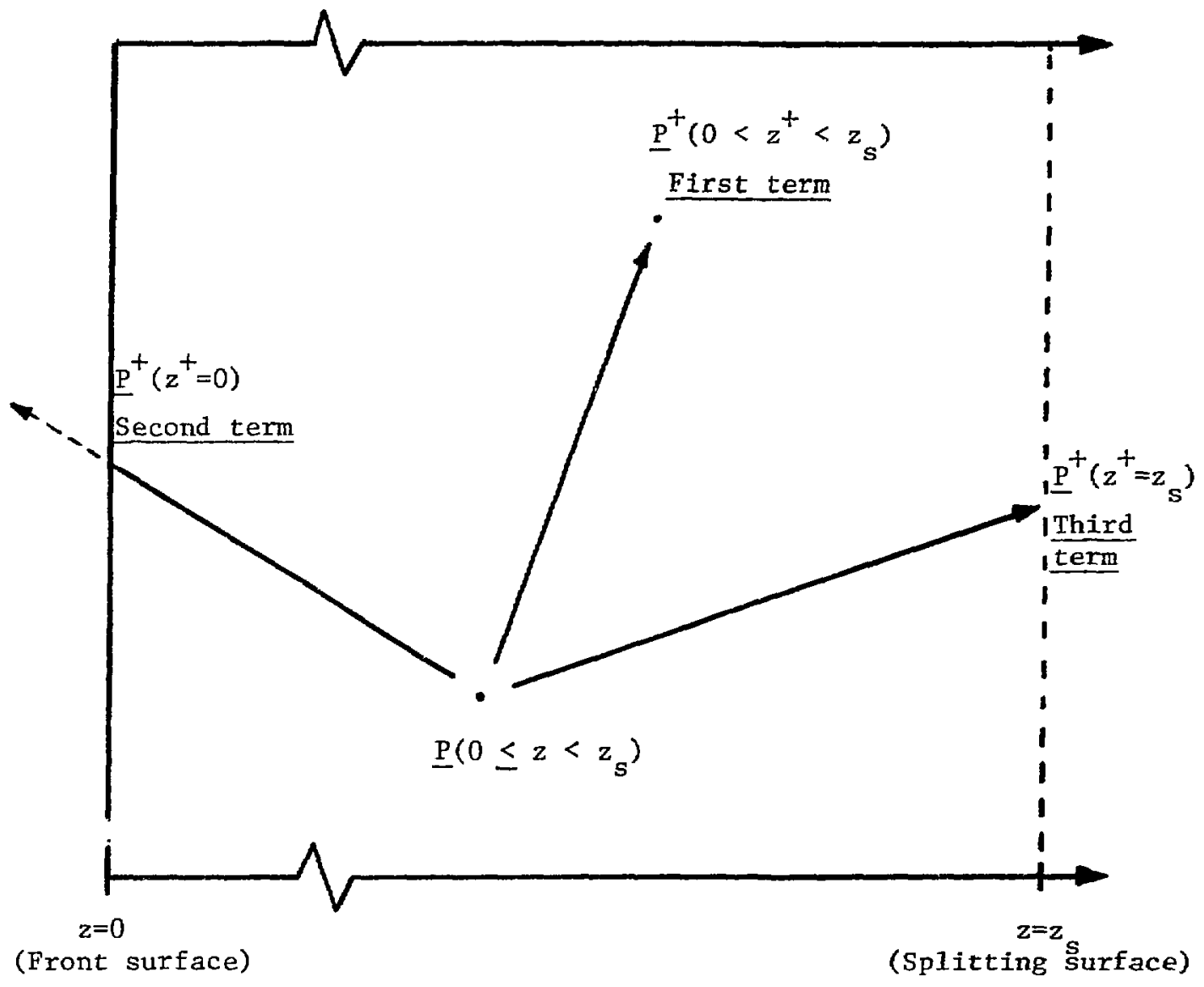

Figure 9. Possible Next Events, Given a Particle Begins its Next Flight to the Left of the Splitting Surface $\left(0 \leq z<z_{s}\right)$.

[References made to terms of Eq.(4.1.1.1).] 
In the most general case, the particle may possibly score at each event, and the score contribution is picked from a score distrifution function appropriate for that particular event. If the next event is terminal, e.g., escape past $z^{+}=0$, the score deposited at the event must be equal to the total score $s$ in order for the event to contribute to $\psi(\underline{P}, s)$ ds. Otherwise, a partial score $s^{\prime}$ deposized at the next evert must be augmented by $s-s^{\prime}$ in future events, in order for the total score over the history (that is, from point $\underline{p}$ on) to be in ds about $s$. Thus, having contributed $s^{-}$at the next event, the particle must contribute to balance $\left(s-s^{\prime}\right)$ before its history is terminated. The flow chart in Fig. 10 illustrates the score accumulation for particles beginning their flight to the left of the splitting surface. Note particularly that in the case of splitting, all the partial scores contributed by the (m) sub-flarticles plus the score resuiting from the splitting event itself must didd up to $s$ in $\mathrm{ds}$.

In the formulation of $\psi_{1}(\underline{P}, s)$, each next event (as depicted in Figs. 9 and 10) contributes a term to the expression of the score accumulation probability:

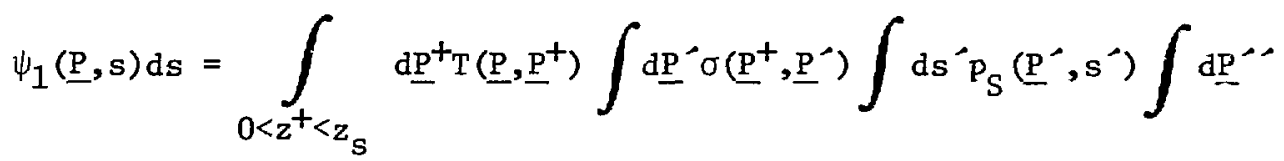

$$
\begin{aligned}
& \times\left[E\left(\underline{P}^{\prime}, \underline{P}^{-\prime}\right) \psi_{1}\left(\underline{P}^{\prime}, s-s^{\prime}\right)\right] d s+\int d \underline{P}^{+} D_{0}\left(\underline{P}, \underline{P}^{+}\right) \int d s^{\prime} p_{E, 0}\left(\underline{P}^{+}, s^{\prime}\right) \delta\left(s-s^{\prime}\right) d s
\end{aligned}
$$

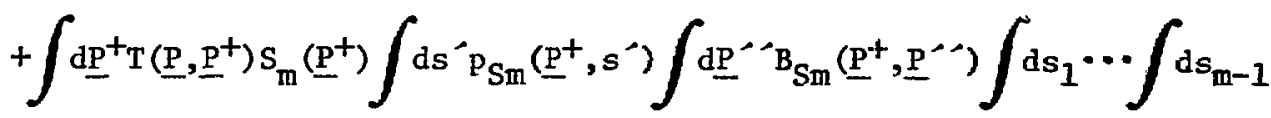

$$
\begin{aligned}
& \times \psi_{z_{s}}\left(\underline{P}^{\cdots}, s_{1}\right) \cdots \psi_{z_{s}}\left(\underline{P}^{\cdots}, s_{m-1}\right) \psi_{z}\left(\underline{P}^{-}, s^{-}\left(s^{\prime}+s_{1}+s_{2}+\cdots+s_{m-1}\right)\right) d s \text {. }
\end{aligned}
$$




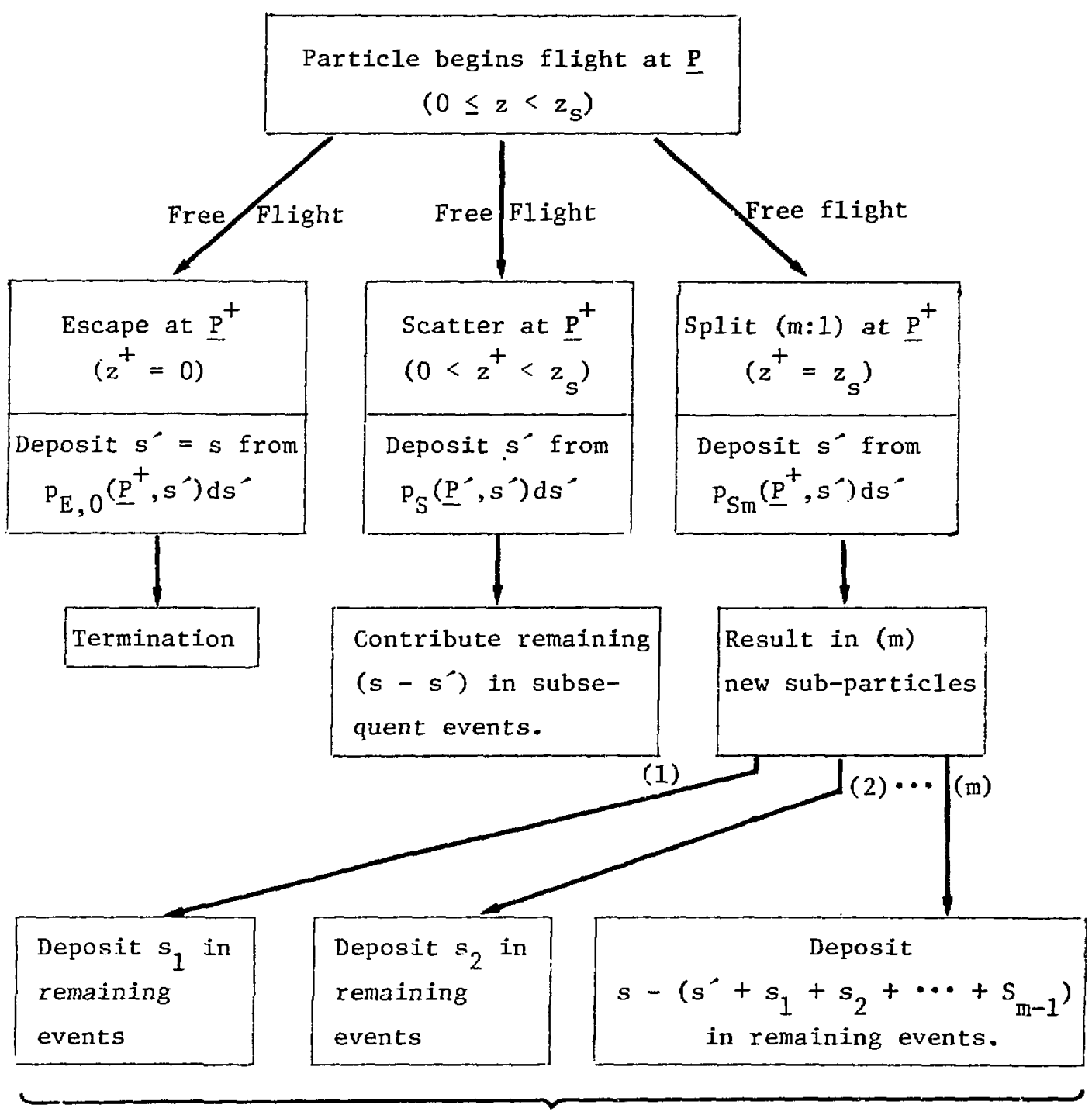

These (m) sub-particles together contribute remaining score $s-s^{\prime}$.

Fig. 10. Deposition of total score $s$, given particle begins flight at $z$, such that $0 \leq z<z^{*}$. 
In effect, Eq. (4.1.1.1) is a mathematical description of the probabilistic chain of events which leads to the eventual deposition (over the particle's remaining history) of a total score $s$ in ds, given the particle starts out at point $\underline{\mathrm{P}}$ in the system. (For a more fundamental and complete discussion of the formulation of score accumulation probabilities, the reader is directed to References 16 and 17 . The notation has been kept almost identical, so that no special translation difficulties should arise.) Each contributing term in the equation is essentially a product of three probabilities:

$$
\begin{aligned}
& {\left[\begin{array}{l}
\text { Probability that the } \\
\text { particular next event occurs } \\
\text { following a free flight } \\
\text { from } \underline{P}
\end{array}\right] \times\left[\begin{array}{l}
\text { Probability that this } \\
\text { event contributes a partial } \\
\text { score s }
\end{array}\right]} \\
& \times\left[\begin{array}{l}
\text { Probability that the particle history } \\
\text { will accumulate the remaining s-s in } \\
\text { all future events. }
\end{array}\right]
\end{aligned}
$$

Thus, term 1 of Eq. (4.1.1.1) formulates the probability of a particle experiencing a free flight from $\underline{P}$ to a collision, making a partial score $s^{\prime}$, scattering from $\underline{P}^{\prime}$ to $\underline{P}^{\prime \prime}$, and scoring the balance of the total score $\mathrm{s}^{-\mathrm{s}^{\prime}}$ in later events. Term 2 expresses the probability of a particle escaping past the front surface and scoring the total $s^{-}=\mathbf{s}$ after a free flight from $\underline{P}$. F:nally, the third term expresses the probability that a particle will experience a free flight to an $m: 1$ splitting event at $\underline{\mathrm{P}}^{+}$, deposit a partial score $\mathrm{s}^{\prime}$ at the event, and the probability that the (m) sub--particles resulting from the split will together score the balance $s^{-s^{-}}$in their subsequent events. 
In the second case to be considered, the particle is considered to be introduced (or more generally, considered to start its next flight) with a starting location to the right of the splitting surface, i.e., $z$ such that $z_{s}<z \leq D$. The possible next events following this flignt are: a scattering collision at $\underline{\mathrm{p}}^{+}$(with $\mathrm{z}^{+}$restricted to the range $\left.z_{s}<z^{+}<D\right)$, an escape past the back surface $\left(z^{+}=D\right)$, or a Russian roulette event at the internal surface $\left(z^{+}=z_{s}\right)$. Figure 11 shows the possible next events for this case, and Fig. 12 illustrates the logic involved in formulating the total score accumulation probability:

$$
\begin{aligned}
& \psi_{2}(\underline{P}, s) d s=\int_{z_{s}<z^{+}<D} \underline{d \underline{p}}^{+} T\left(\underline{P}, \underline{P}^{+}\right) \int \underline{\mathrm{P}}^{\prime} \sigma\left(\underline{\mathrm{P}}^{+}, \underline{\mathrm{p}}^{\prime}\right) \int \mathrm{ds^{ \prime }} \mathrm{p}_{\mathrm{s}}\left(\underline{\mathrm{P}}^{\prime}, \mathrm{s}^{\prime}\right) \int \mathrm{d} \underline{\mathrm{P}}^{-} \\
& \times\left[E\left(\underline{P}^{-}, \underline{P}^{-}\right) \psi_{2}\left(\underline{P}^{-}, s^{-s^{\prime}}\right)\right] d s+\int d \underline{P}^{+} D_{T}\left(\underline{P}, \underline{P}^{+}\right) \int d s^{\prime} P_{E, D}\left(\underline{P}^{+}, s^{\prime}\right) \delta\left(s^{-s^{\prime}}\right) d s \\
& +\int d \underline{P}^{+} T\left(\underline{P}, \underline{P}^{+}\right) R_{m}\left(\underline{P}^{+}\right) \int d s^{\prime} p_{R}\left(\underline{P}^{+}, s^{\prime}\right) \int d \underline{P}^{-}{ }^{-} B_{0 m}\left(\xi_{m} ; \underline{p}^{+}, \underline{P}^{\prime \prime}\right) \psi_{z}\left(\underline{P}^{-\prime} \cdot s-s^{\prime}\right) d s .
\end{aligned}
$$

Equation (4.1.1.2) thus formulates the probability of all event chains leading to the eventual deposition of a total score $s$ in ds over the remaining particle history, given the particle is introduced at $\underline{P}$ with $z_{s}<z \leq D$. Term 1 expresses the probability of a particle experiencing a free flight from $\underline{P}$ to a collision, making a partial score $\mathbf{s}^{\prime}$, scattering from $\underline{\mathrm{P}}^{\prime}$ to $\underline{\mathrm{P}}^{\prime \prime}$, and proceeding via subsequent events to score the balance of the total score $s^{-s^{\circ}}$. Of course, the term actually represents the integral over all such event chains. Term 2, 1ikewise, formulates the probability that the particle will escape past the back 


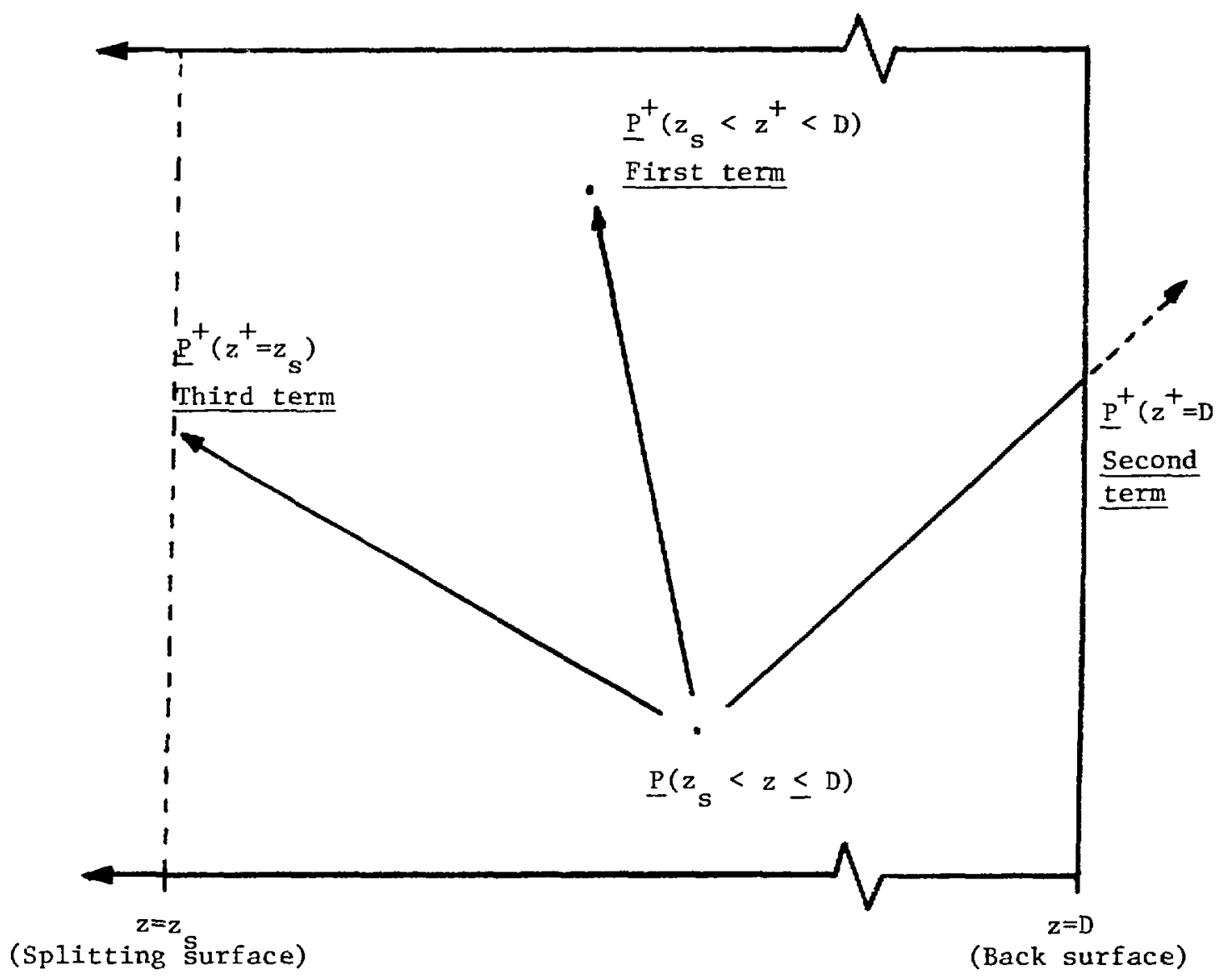

Figure 11. Fossible Next Events, Given a Particle Begins its Next Flight to the Right of the Splitting Surface $\left(z_{s}<z \leq D\right)$

[References made to terms of Eq.(4.1.1.2).] 


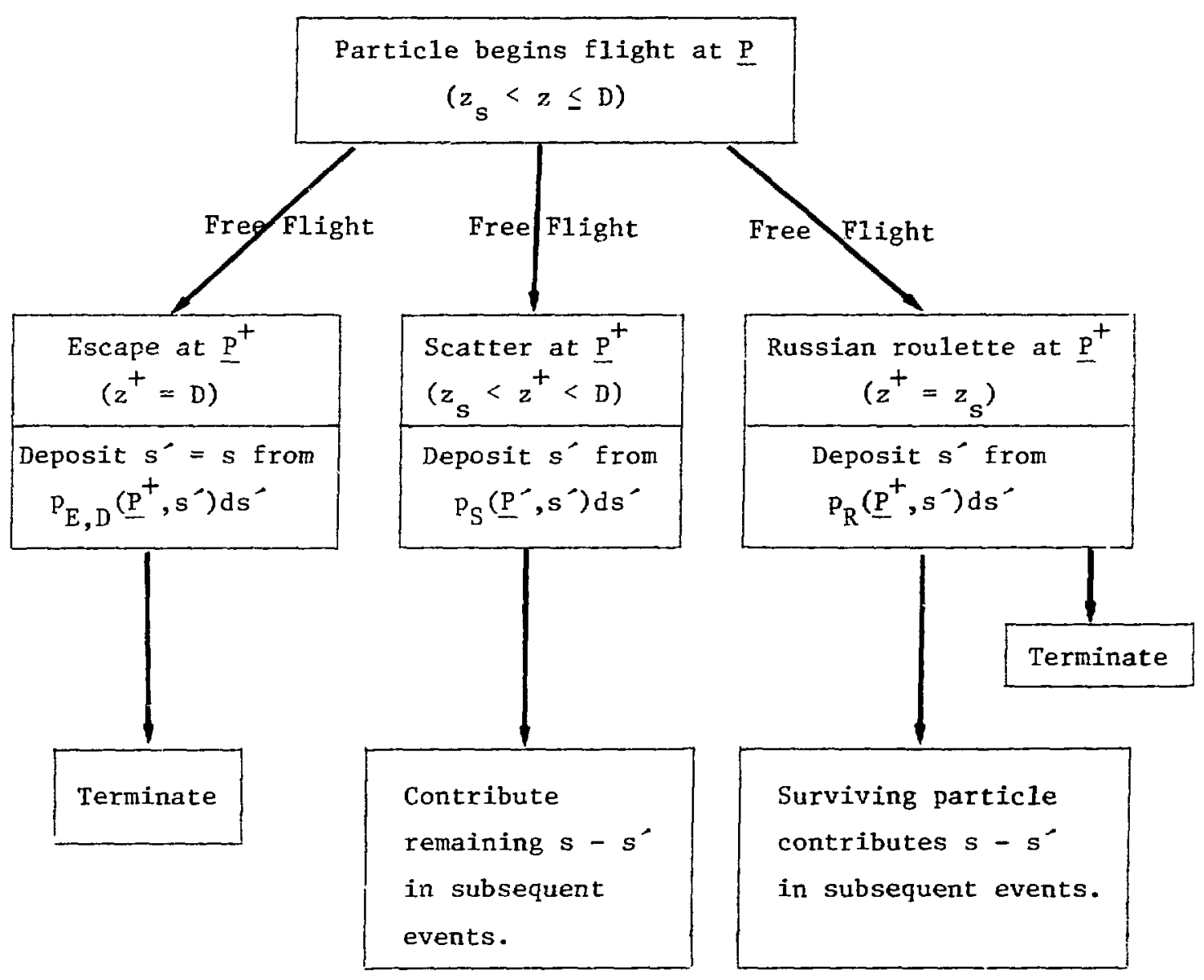

Fig. 12. Deposition of total score $s$, given particle begins flight at $z$, such that $z_{\mathbf{s}}<z \leq D$. 
surface and score $s^{\prime}=s$ after a free flight from $\underline{P}$. Finally, the last term expresses the probability that a particle will experience a Russian roulette event at $\underline{P}^{+}$after a free flight from $\underline{P}$, deposit $s^{\prime}$ at the event, and the probability that the surviving particle will continue to score the remaining $s-s^{\prime}$ in future events.

Finally, the third case is considered in which particles continue their flight from the splitting surface, i.e., with $z=z_{s}$. The possible next events in this case are: a scattering collision at $\underline{\mathrm{P}}^{+}$(where $0<z^{+}<z_{s}$ or $\left.z_{s}<z^{+}<D\right)$, an escape past the front surface, or an escape past the back surface (see Figs. 13 and 14). As in the previous two cases, the score accumulation probability is written by summing over several terms, each associated with the probability of an event chain begun by a particular next event following a free flight of the particle from $z_{s}$ :

$$
\begin{aligned}
& \psi_{z_{s}}(\underline{P}, s) d s=\int_{0<z^{+}<z_{s}} d \underline{P}^{+} T\left(\underline{P}, \underline{P}^{+}\right) \int d \underline{P}^{\circ} \sigma\left(\underline{P}^{+}, \underline{P}^{\prime}\right) \int d s^{\prime} p_{S}\left(\underline{P}^{\prime}, s^{\prime}\right) \int d \underline{P}^{-\infty}
\end{aligned}
$$

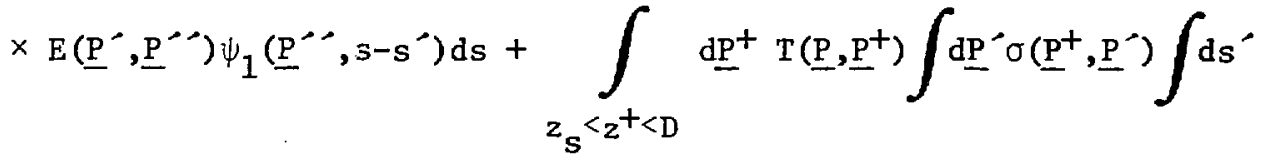

$$
\begin{aligned}
& \times p_{S}\left(\underline{P}^{\prime}, s^{\prime}\right) \int d \underline{P}^{-} E\left(\underline{P}^{\prime}, \underline{P}^{\prime}\right) \psi_{2}\left(\underline{P}^{\prime}, s^{-s^{\prime}}\right) d s+\int d \underline{P}^{+} D_{0}\left(\underline{P}, \underline{P}^{+}\right) \int d s^{\prime} \\
& \times \mathrm{p}_{E, 0}\left(\underline{\mathrm{P}}^{+}, \mathrm{s}^{\prime}\right) \delta\left(\mathrm{s}-\mathrm{s}^{\prime}\right)+\int \mathrm{dP}^{+} \mathrm{D}_{\mathrm{T}}\left(\underline{\mathrm{P}}, \underline{\mathrm{P}}^{+}\right) \int \mathrm{d} \mathrm{s}^{\prime} \mathrm{p}_{\mathrm{E}, \mathrm{D}}\left(\underline{\mathrm{P}}^{+}, \mathrm{s}^{\prime}\right) \delta\left(\mathrm{s}-\mathrm{s}^{\prime}\right) \mathrm{ds}
\end{aligned}
$$




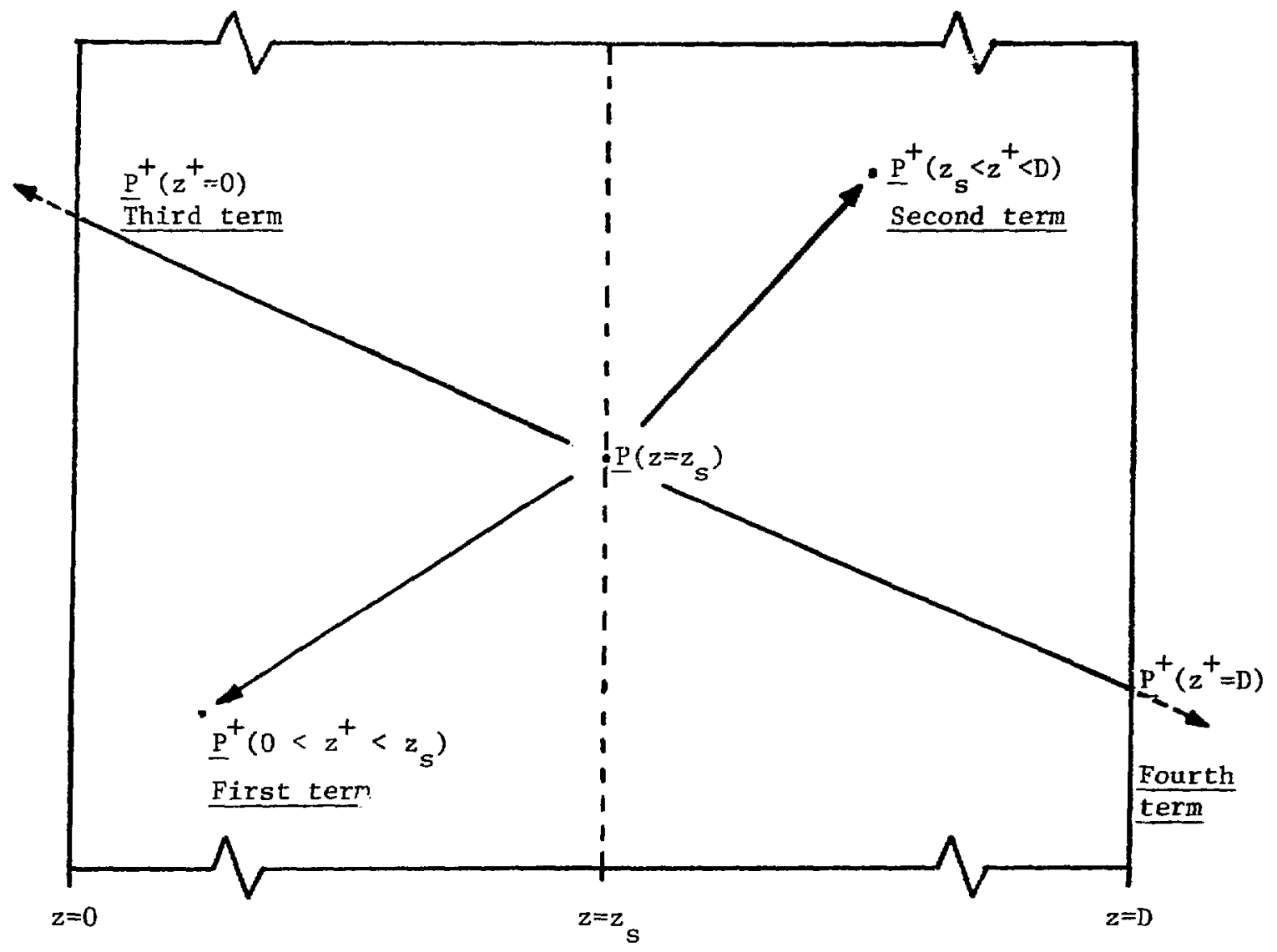

Figure 13. Possible Next Events, Given a Particle Begins its Next Flight at the Splitting Surface $\left(z=z_{s}\right)$.

[Refernces made to terms of $\mathrm{Eq} \cdot(4 \cdot 1.1 .3)$. 


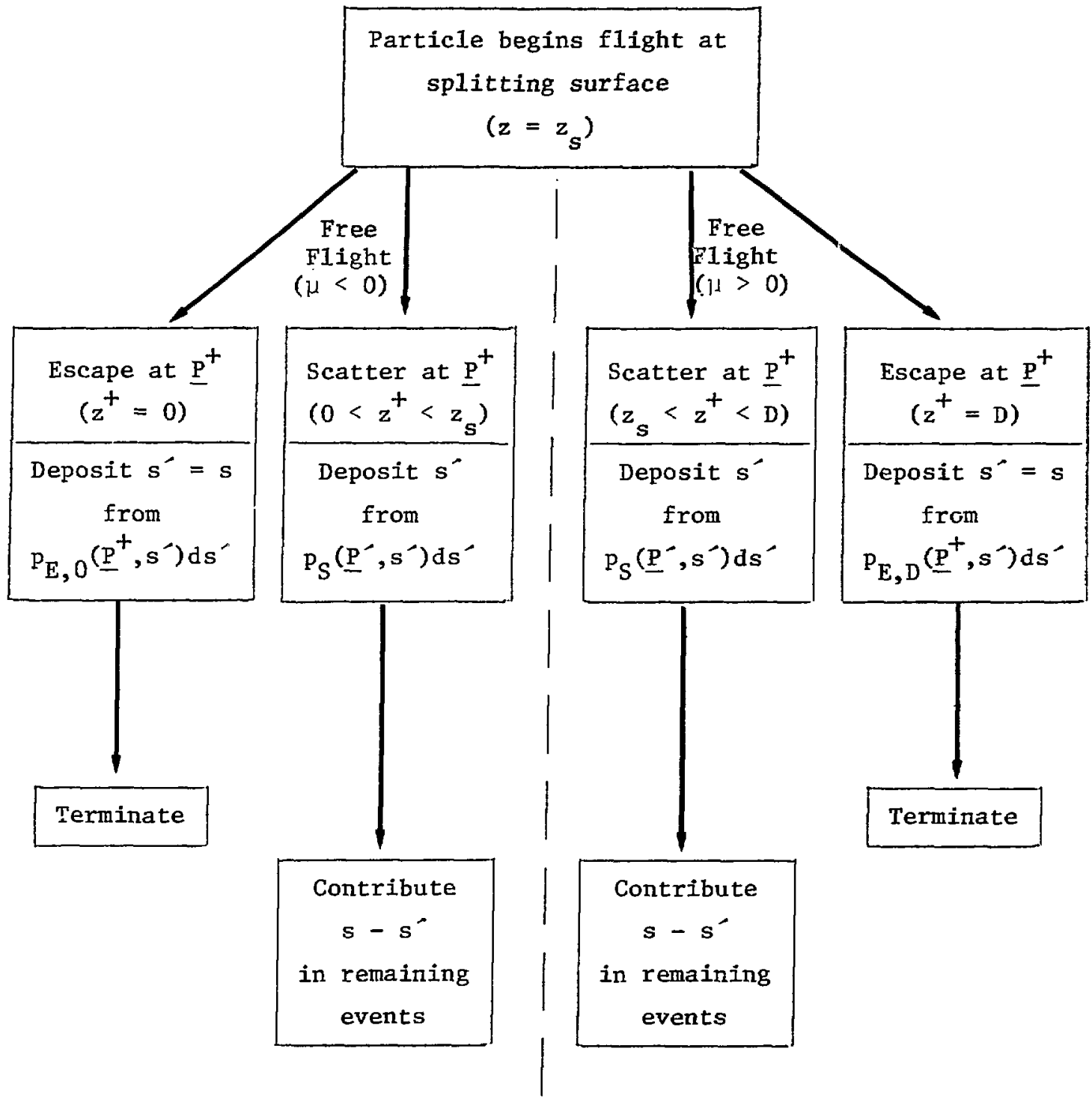

Figure 14. Deposition of total score s, given particle begins (continues) flight at splitting surface, $z=z_{s}$. 
Due to the definitions of $\mathrm{T}\left(\underline{\mathrm{P}}, \underline{\mathrm{P}}^{+}\right), \mathrm{D}_{0}\left(\underline{\mathrm{P}}, \underline{\mathrm{P}}^{+}\right)$, and $\mathrm{D}_{\mathrm{T}}\left(\underline{\mathrm{P}}, \underline{\mathrm{P}}^{+}\right)$as well as the restrictions of $\mathrm{z}^{+}$to the ranges indicated in Eq. (4.1.1.3), the first and third terms in the above equation drop out for $\underline{P}$ with $\mu>0$. For similar reasons, the second and fourth terms are eliminated for $\underline{p}$ with $\mu<0$. [It should be recalled that in the formulation of $\psi_{z_{s}}(\underline{P}, s)$, the starting location is always $z=z_{s}$ ].

Thus, the coupled integral equations [Eqs. (4.1.1.1), (4.1.1.2), and (4.1.1.3)] together define the score accumulation probability over the entire range of starting positions $z$ :

$\psi(\underline{P}, s) d s=\left\{\begin{array}{l}\psi_{1}(\underline{P}, s) d s \text { for } \underline{P} \text { such that } 0 \leq z<z_{S}, \\ \psi_{z_{s}}(\underline{P}, s) d s \text { for } \underline{P} \text { such that } z=z_{S}, \text { and } \\ \psi_{2}(\underline{P}, s) d s \text { for } \underline{P} \text { such that } z_{s}<z \leq D .\end{array}\right.$ $(4.1 .1 .4)$

The next step in this development is to formulate the $r$-th moment of the Monte Carlo score over the score accumulation probability (given the particle begins its flight at $\underline{\mathrm{P}}$ ), or

$$
\mathrm{M}_{\mathrm{r}}(\underline{\mathrm{P}}) \equiv \int \psi(\underline{\mathrm{P}}, \mathrm{s}) \mathrm{s}^{\mathrm{r}} \mathrm{ds}
$$

At this point, a special remark may also be made in regard to particles of zero weight. Since the tally score is proportional to particle weight, a particle of weight zero cannot contribute a non-zero score to the tally (in fact, the particle is terminated). Thus, the following definition is also made:

$$
\mathcal{K}_{r}(w=0)=0
$$


Since the score accumulation probability has been written for three different cases, depending on where (relative to the splitting surface) the particle is considered to start its flight, the $r$-th moment must also be formulated for these three cases. Specifically, Eqs. (4.1.1.1), $(4.1 .1 .2)$, and $(4.1 .1 .3)$ are multipiled by the $r-t h$ power of the score $\mathrm{s}^{\mathrm{r}}$ and integrated over $\mathrm{ds}$ to yield

$$
M_{r} \underline{(P)} \equiv \begin{cases}M_{r, 1}(\underline{P}) & \text { for } \underline{P} \text { with } 0 \leq z<z_{s} \\ M_{r, z} \stackrel{(P)}{s} & \text { for } \underline{P} \text { with } z=z_{s} \text {, and } \\ M_{r, 2}(\underline{P}) & \text { for } \underline{P} \text { with } z_{s}<z \leq D .\end{cases}
$$

Since the prediction of sample variance requires only the first two moments of the score $(r=1,2)$, only these two cases must be considered for the purposes of this work.

The mathematical details of the development of these equations are contained in Appendix B. The resultant formulations for the first two moments of the slab transmission score (the particular tally considered in this work) are rewritten here from Eqs. (B.1.26); (B.1.27), and (B.1.28). In writing the moment equations here, the weight-modification factors have been separated from the arguments of the $r$-th moments (see Appendix D). Thus, for particle starting locations $z$, such that $0 \leq z<z_{s}:$ 


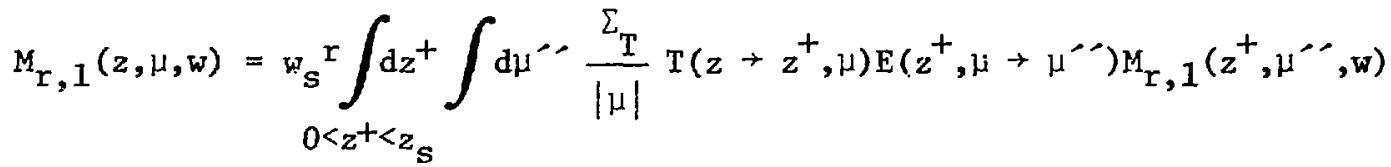

$$
\begin{aligned}
& +\underline{1}(\mu) \cdot T\left(z \rightarrow z_{s}, \mu\right) \cdot \mathrm{m}^{1-r} \cdot M_{r, z_{s}}\left(z_{s}, \mu, w\right)+Q_{r, 1}(z, \mu, w), \quad \text { 't.1.1.8a) } \\
& \mathrm{Q}_{1,1}(z, \mu, \mathrm{w})=0
\end{aligned}
$$

For $z_{S}<z \leq D$ :

$$
\begin{aligned}
& M_{r, 2}(z, \mu, w)=w_{s}^{r} \int_{z_{s}<z^{+}<D} d^{+} \int d_{\mu} \cdots \frac{\bar{\Sigma}_{T}}{|\mu|} T\left(z \rightarrow z^{+}, \mu\right) E\left(z^{+}, \mu \rightarrow \mu^{-\cdots}\right) \\
& \quad \times M_{r, 2}\left(z^{+}, \mu^{-}, w\right)+[1-H(\mu)] \cdot T\left(z \rightarrow z_{s}, \mu\right) \cdot m^{r-1} \cdot M_{r, z_{s}}\left(z_{s}, \mu, w\right) \\
& +Q_{r, 2}(z, \mu, w),
\end{aligned}
$$

where

$$
Q_{1,2}(z, \mu, w)=H(\mu) \cdot T(z \rightarrow D, \mu) \cdot w
$$

and

$$
Q_{2,2}(z, \mu, w)=H(\mu) \cdot T(z \rightarrow D, \mu) \cdot w^{2} .
$$

Finally, for $z=z_{s}$ : 


$$
\begin{aligned}
& M_{r, z_{s}}\left(z_{s}, \mu, w\right)=w_{s}^{r} \int_{0<z^{+<z_{S}}} \mathrm{dz}^{+} \int \mathrm{d} \mu^{-}-\frac{\Sigma_{\mathrm{T}}}{|\mu|} \mathrm{T}\left(z_{\mathrm{s}}+z^{+}, \mu\right) E\left(z^{+}, \mu \rightarrow \mu^{-\infty}\right) \\
& \times M_{r, 1}\left(z^{+}, \mu^{\cdots}, w\right)+w_{S}^{r} \int_{z_{S}<z^{+}<D} d z^{+} \int d \mu^{-} \frac{\Sigma_{T}}{|\mu|} T\left(z_{S} \rightarrow z^{+}, \mu\right) E\left(z^{+}, \mu \rightarrow \mu^{\prime}\right) \\
& \times M_{r, 2}\left(z^{+}, \mu^{\prime}, w\right)+Q_{r, z_{s}}\left(z_{s}, \mu, w\right),
\end{aligned}
$$

where

$$
\mathrm{Q}_{1, z_{s}}\left(z_{s}, \mu, w\right)=H(\mu) \cdot T\left(z_{s} \rightarrow D, \mu\right) \cdot w
$$

and

$$
\mathrm{Q}_{2, z_{S}}\left(z_{S}, \mu, w\right)=H(\mu) \cdot T\left(z_{s} \rightarrow D, \mu\right) \cdot w^{2}
$$

As indicated by the discussion in Appendix B, various terms in the above sets of equations are eliminated for either $\mu<0$ or $\mu>0$ due to the zestrictions inherent in the definition of the transmission probability or the presence of the Heaviside function on $\mu$.

The behavior of the solution functions at the internal splitting surface $z_{S}$ is investigated in detail in Appendix B. The results are restated here. The first moment, i.e., $\mathrm{M}_{1,1}$ or $\mathrm{M}_{1,2}$, is found to be continuous across the splitting surface. In fact, a single integral equation may be written for the first moment of the transmission score, applicable over the entire range of starting locations $z$ (that is, for $0 \leq z \leq D):$

$$
\begin{aligned}
\mathrm{M}_{\mathrm{L}}(z, \mu, w) & =\mathrm{w}_{\mathrm{S}} \int_{0<\mathrm{z}^{+}<\mathrm{D}} \mathrm{d} \mathrm{z}^{+} \int \mathrm{d} \mu^{\prime \prime} \mathrm{K}\left(z, \mu \rightarrow z^{+}, \mu^{\prime \prime}\right) \mathrm{M}_{1}\left(z^{+}, \mu^{\prime}, w\right) \\
& +\mathrm{H}(\mu) \cdot \mathrm{T}(z \rightarrow \mathrm{D}, \mu) \cdot \mathrm{w},
\end{aligned}
$$


where the transition kernel is as defined in Appendix B: i.e.,

$$
K\left(z, \mu \rightarrow z^{+}, \mu^{-\prime}\right) \equiv \frac{\Sigma_{T}}{|\mu|} T\left(z \rightarrow z^{+}, \mu\right) E\left(z^{+}, \mu \rightarrow \mu^{-\prime}\right) \text {. }
$$

Thus, the expected value (first moment) of the Monte Carlo score is not affected by the processes of splitting and Russian roulette. This is as it should be: variance reduction techniques should not result in a bias of the Monte Carlo estimate (tally). In fact, Eq. (4.1.1.11) is identical to the equation that would be derived if only survival biasing (but no splitting or Russian roulette) were assumed to be implemented in the Monte Sarlo calculation.

The same is not true for the second moment of the transmission score. A discontinuity condition may be characterized for the second moment solution function at $z=z_{s}$ (see Appendix B):

for $\mu<0$,

$$
M_{2}^{-}\left(z_{s}, \mu, w\right)=\frac{1}{m} \cdot M_{2}^{+}\left(z_{s}, \mu, w\right),
$$

and for $\mu>0$,

$$
M_{2}^{+}\left(z_{s}, \mu, w\right)=m \cdot M_{2}^{-}\left(z_{s}, \mu, w\right)-(m-1)\left[M_{1, z_{s}}\left(z_{s}, \mu, w\right)\right]^{2} \cdot(4 \cdot 1 \cdot 1 \cdot 14)
$$

In the above pajis of equations

$$
\mathrm{M}_{2}^{-}\left(z_{s}, \mu, w\right) \equiv \lim _{z \rightarrow z_{s-}} M_{2,1}(z, \mu, w)
$$

and $M_{2}^{+}\left(z_{s}, \mu, w\right) \equiv \lim _{z \rightarrow z_{s+}} M_{2,2}(z, \mu, w)$ 
Although rigorously derived in Appendix $B_{3}$ the discontinuity conditions stated in Eqs. (4.1.1.13) and (4.1.1.14) are intuitively plausible.

For shorthand purposes, let the second moment for particles starting at $z_{s}$ and about to be split be represented by $\left\langle x^{2}>-\equiv M_{2}\left(z_{s}, \mu, w\right)\right.$ for $\mu>0$. Also, $\left\langle\mathrm{x}^{2}\right\rangle^{+} \equiv \mathrm{M}_{2}^{+}(z, \mu, w)$ for $\mu>0$ represents the second moment for particles starting at the surface, but not subjected to splitting. The first moment for particles starting at $z_{s}$ is represented as $\langle\mathrm{x}\rangle \equiv \mathrm{M}_{1, z_{s}}\left(z_{s}, \mu, w\right)$. Now, Eq. (4.1.1.14) may be written:

$$
\left\langle\mathrm{x}^{2}>-=\frac{1}{\mathrm{~m}}\left\langle\mathrm{x}^{2}>+-\frac{1}{\mathrm{~m}}\langle\mathrm{x}\rangle^{2}+\langle\mathrm{x}\rangle^{2} .\right.\right.
$$

The sample variance for particles about to be split may be formulated

$$
\sigma_{s}^{2} \equiv\left\langle x^{2}\right\rangle^{-}-\langle x\rangle^{2}=\frac{1}{m}\left[\left\langle x^{2}\right\rangle^{+}-\langle x\rangle^{2}\right] .
$$

Thus, (m:1) splitting results in an $\mathrm{m}$-fold reduction of the sample variance, given particles start out at the splitting surface. This is a generalization of the condition found by Everett and Cashwe11:12 splitting $(\mathrm{m}: 1)$ at the source resulted in an $\mathrm{m}$-fold reduction in sample variance. In effect, the same relative error in the calculation may be obtained by simply increasing by a factor $m$ the number of histories started.

For the case of Russian roulette $\left(\mu<0\right.$ at $\left.z_{s}\right),\left\langle x^{2}\right\rangle^{+}$now represents the second moment for particles starting at $z_{s}$ and about to be subjected to Russian roulette. The second moment for particles starting at $\mathbf{z}_{\mathbf{s}}$ but not participating in the Russian roulette game is expressed as $\left\langle x^{2}\right\rangle-$. Equation $(4 \cdot 1.1 .13)$ can be rewritten as 


$$
\left\langle\mathrm{x}^{2}\right\rangle^{+}=\mathrm{m} \cdot\left\langle\mathrm{x}^{2}\right\rangle^{-} \cdot
$$

The same condition may be arrived at by a more fundamental argument. Recall that pariicles survive the roulette game with probability $\xi_{\mathrm{m}}=1 / \mathrm{m}$, and the weight of surviving particles is increased by a factor of $\mathrm{m}$. Thus, for a particle of weight $w$ introduced at $z_{s}$ and about to undergo Russian roulette, the probability of scoring $s$ in ds in the course of its remaining history is equal to the probability of surviving the game times the score accumulation probability (given the particle starts at $z_{s}$ with weight $\left.\mathrm{nnw}\right)$. The expected value of the square of the score may then be formulated as

$$
\left\langle\mathrm{x}^{2}\right\rangle^{+}=\int \frac{1}{\mathrm{~m}} \cdot \psi\left(\mathrm{z}_{\mathrm{s}}, \mu<0, \mathrm{mw}, \mathrm{s}\right) \mathrm{s}^{2} \mathrm{ds} .
$$

However, in the Monte Carlo simulation being considered in this work, the particle weight has no effect on the sampling of any probability densities during the random walk. A1so, the score in this transport problem (transmission through the $s$ lab) is proportional to the particle weight--in fact, it is the particle weight. Thereiore, it is possible to write

$$
\psi\left(\underline{R}, m_{w} w, s\right) d s=\psi\left(\underline{R}, w, \frac{s}{m}\right) d\left(\frac{s}{m}\right)
$$

Substituting Eq. $(4 \cdot 1 \cdot 1.20)$ into Eq. (4.1.1.19):

$$
\begin{aligned}
\left.<\mathrm{x}^{2}\right\rangle^{+} & =\frac{1}{\mathrm{~m}} \int \psi\left(\mathrm{z}_{\mathrm{s}}, \mu<0, \mathrm{w}, \frac{\mathrm{s}}{\mathrm{m}}\right) \mathrm{s}^{2} \mathrm{~d}\left(\frac{\mathrm{s}}{\mathrm{m}}\right) \\
& =\frac{1}{\mathrm{~m}} \cdot \mathrm{m}^{2} \int \psi\left(\mathrm{z}_{\mathrm{s}}, \mu<0, \mathrm{w}, \frac{\mathrm{s}}{\mathrm{m}}\right)\left(\frac{\mathrm{s}}{\mathrm{m}}\right)^{2} \mathrm{~d}\left(\frac{\mathrm{s}}{\mathrm{m}}\right),
\end{aligned}
$$




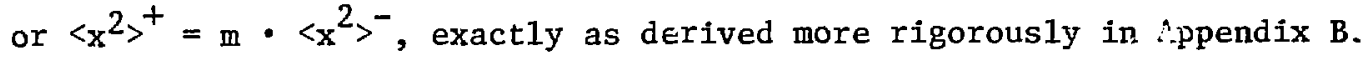
By itself, the Russian roulette game results in a discontinuous gain in sample variance for the Monte Carlo calculation.

As expected, the discontinuities characterized in Eqs. (4.1.1.13) and (4.1.1.14) disappear when cell importances on either side of the splitting surface are equal, i.e., for $m=1$. In this case, the second moment of the transmission score is continuous across $z=z_{s}$.

\subsubsection{Conversion to Integro-Differential Form}

In order to provide practical significance to the theoretical development presented in the previous section, it is important to be able to solve the $r$-th momert equations $(r=1$ and 2 ), allowing for a deterministic prediction of the sample variance. The linear integral moment equations presented in Section 4.1.1 may be converted to an integro-differential form, and in this form the equations are amenable to a standard numerical solution technique--namely, a discrete ordinates $\left(s_{n}\right)$ solution. The conversion of the integral equations to an integrodifferential formulation is presented in this section.

The development here hinges on an important identity, the proof of which is presented in Appendix $\mathrm{E}$ :

$$
\mathrm{R}_{\mathrm{z}}^{+} \mathrm{C}\left(z \rightarrow \mathrm{z}^{\prime}, \mu\right)=\delta\left(z-\mathrm{z}^{\prime}\right)
$$

Here $\mathrm{R}_{2}^{+}$represents the adjoint differential transport operator for the one-group transport problem in one-dimensional slab geometry,

$$
\mathrm{R}_{z}^{+}=\left(-\frac{1}{\Sigma_{\mathrm{T}}} \mu \frac{\partial}{\partial z}+1\right),
$$


and $C\left(z \rightarrow z^{\bullet}, \mu\right)$ is the transport kernel, which is written in terms of the transmission probability:

$$
C\left(z \rightarrow z^{\prime}, \mu\right) d z^{\prime}=\frac{\Sigma_{T}}{|\mu|} \cdot T\left(z \rightarrow z^{\prime}, \mu\right) d z^{\prime}
$$

Thus $\mathrm{C}\left(z \rightarrow z^{\prime}, 1\right) \mathrm{d} z^{\prime}$ represents the probability that a particle starting out at $z$ with direction $\mu$ will collide in $\mathrm{d}^{-}$about $z^{-}$before making any other collisions.

If follows from Eqs. (4.1.2.1) and (4.1.2.3) that we also have the following identity:

$$
\mathrm{R}_{\mathrm{z}}^{+} \mathrm{T}\left(z \rightarrow \mathrm{z}^{\prime}, \mu\right)=\frac{|\mu|}{\Sigma_{\mathrm{T}}} \delta\left(z-\mathrm{z}^{\prime}\right)
$$

These identities will be applied in the course of the development below. The conversion of the first moment $(r=1)$ equation proceeds by first defining

$$
\mathrm{G}\left(\mathrm{z}^{+}, \mu, w\right) \equiv \int \mathrm{d} \mu^{-} \mathrm{E}\left(z^{+}, \mu \rightarrow \mu^{-}\right) \mathrm{M}_{1}\left(z^{+}, \mu^{-}, w\right)
$$

Using Eqs. (4.1.2.5) and (4.1.1.12), Eq. (4.1.1.11) may be rewritten in terms of $G$ as follows:

$$
\begin{aligned}
M_{1}(z, \mu, w) & =w_{S} \int_{0<z^{+}<D} d z^{+} \frac{\Sigma_{T}}{|\mu|} T\left(z \rightarrow z^{+}, \mu\right) G\left(z^{+}, \mu, w\right) \\
& +H(\mu) T(z \rightarrow D, \mu) \cdot w .
\end{aligned}
$$

However, 


$$
G(z, \mu, w)=\int_{0<z^{+}<D} \delta\left(z-z^{+}\right) G\left(z^{+}, \mu, w\right) d z^{+},
$$

since $0 \leq z \leq D$. So by employing Eq. (4.1.2.1) and noting that $R_{z}^{+}$is independent of $\mathrm{z}^{+}$:

$$
G(z, \mu, w)=R_{z}^{+} \int_{0<z^{+}<D} C\left(z \rightarrow z^{+}, \mu\right) G\left(z^{+}, \mu, w\right) d z^{+}
$$

Substitution of Eq. (4.1.2.3) into Eq. (4.1.2.6) and rearrangement yields

$$
\int_{0<z^{+}<D} d z^{+} C\left(z \rightarrow z^{+}, \mu\right) G\left(z^{+}, \mu, w\right)=\frac{1}{w_{s}}\left[M_{1}(z, \mu, w)-H(\mu) T(z \rightarrow D, \mu) \cdot w\right] .
$$

The operator $\mathrm{R}_{\mathrm{z}}^{+}$is then applied to Eq. $(4.1 .2 .9)$, and Eq. $(4.1 .2 .8)$ is used to write

$G(z, \mu, w)=\frac{1}{w_{S}} R_{z}^{+}\left[M_{1}(z, \mu, w)-H(\mu) T(z \rightarrow D, \mu) \cdot w\right]$.

By applying the definition of $G(z, \mu, w)$ as stated in Eq. (4.1.2.5), the above equation is written

$$
\int \mathrm{d} \mu{ }^{-} E\left(z, \mu \rightarrow \mu^{\prime}\right) M_{1}\left(z, \mu^{\cdots}, w\right)=\frac{1}{w_{S}} R_{z}^{+}\left[M_{1}(z, \mu, w)-H(\mu) T(z \rightarrow D, \mu) \cdot w\right],
$$

and rearranging: 
$\mathrm{R}_{z}^{+} \mathrm{M}_{1}(z, \mu, w)=\mathrm{w}_{s} \int \mathrm{d} \mu \cdots \mathrm{E}\left(z, \mu \rightarrow \mu^{-\prime}\right) \mathrm{M}_{1}\left(z, \mu{ }^{-}, w\right)+H(\mu) \mathrm{R}_{z}^{+} \mathrm{T}(z \rightarrow D, \mu) \cdot \mathrm{w}$.

By employing the identity $(4.1 .2 .4)$ in Eq. (4.1.2.12), the equation may be rewritten as

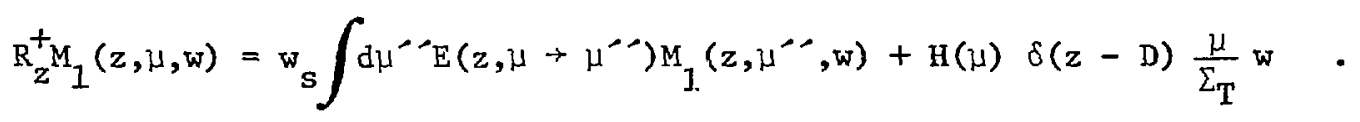

Finally, substitution for $R_{z}^{+}$and $w_{s}$ yields

$-\mu \frac{\partial}{\partial z} F_{1}(z, \mu, w)+\Sigma_{T} F_{I}(z, \mu, w)=\int d \mu^{\prime} \Sigma_{s} \cdot E\left(z, \mu+\mu^{\prime \prime}\right) F_{1}\left(z, \mu^{\prime}, w\right)$

$$
+H(\mu) \delta(z-D) \cdot \frac{\mu}{\Sigma_{T}} w
$$

The solution function $\mathrm{F}_{1}$ has been defined

$$
\mathrm{F}_{1}(\mathrm{z}, \mu, w) \equiv \frac{1}{\Sigma_{\mathrm{T}}} \mathrm{M}_{1}(\mathrm{z}, \mu, w)
$$

Equation (4.1.2.14) is an adjoint formulation of the one-speed Boltzmann transport equation. More specifically, Eq. (4.1.2.14) is an equation for the Green's function. A solution may be obtained by solving the homogeneous form of the equation subject to a boundary solirce (non-zero boundary condition) at $z=D^{20}$. For this particular problem, the condition is written

$$
\begin{aligned}
& M_{1}(D, \mu>0,1)=1 \\
& F_{1}(D, \mu>0,1)=1 / \Sigma_{T} .
\end{aligned}
$$

or 
The expected value of the transmission score, given a particle starts out at $z=D$ in a positive direction with starting weight unity, is identically one. Also, a zero boundary condition exists at $z=0$, i.e.,

$$
M_{1}(0, \mu<0,1)=0
$$

or

$$
\mathrm{F}_{1}(0, \mu<0,1)=0
$$

The transformation of the second moment equations follows the same procedure as applied to the first moment, with some notable differences. The conversion of the integral equation for $\mathrm{M}_{2,1}$ will be discussed first. Equations (4.1.1.8a and c) are rewritten here for $r=2$. Recall that particle starting locations are restricted to $0 \leq z<z_{s}$ in this case.

$$
\begin{aligned}
M_{2,1}(z, \mu, w) & =w_{S}^{2} \int_{0<z^{+}<z_{s}} d^{+} \int d \mu^{\prime} \frac{\sum_{T}}{|\mu|} T\left(z \rightarrow z^{+}, \mu\right) E\left(z^{+}, \mu \rightarrow \mu^{\prime \prime}\right) \\
& \times M_{2,1}\left(z^{+}, \mu^{-,}, w\right)+S_{2,1}(z, \mu, w)
\end{aligned}
$$

where

$$
\begin{aligned}
S_{2,1}(z, \mu, w) & =H(\mu) \cdot \frac{1}{m} \cdot T\left(z \rightarrow z_{s}, \mu\right) M_{2, z_{s}}\left(z_{s}, \mu, w\right) \\
& +H(\mu) \cdot\left(\frac{m-1}{m}\right) \cdot T\left(z \rightarrow z_{s}, \mu\right)\left[M_{1, z_{s}}\left(z_{s}, \mu, w\right)\right]^{2} .
\end{aligned}
$$

The transformation of Eq. (4.1.2.18) to integro-differential form follows the same steps as already detailed in the transformation of Eq. (4.1.1.11). The development encompassing Eqs. (4.1.2.5) through 
(4.1.2.12) is repeated, however now $M_{2,1}(z, \mu, w)$ replaces $M_{1}(z, \mu, w)$ in

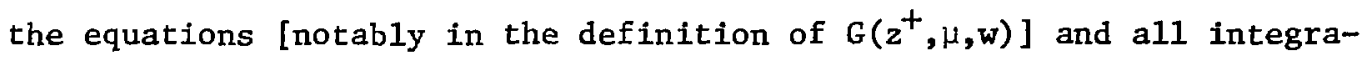
tions over $\mathrm{dz}^{+}$are restricted to the range of starting locations $0<z^{+}<z_{s}$. Also, $S_{2,1}(z, \mu, w)$ replaces the term $H(\mu) \cdot T(z \rightarrow D, \mu) \cdot w$ in Eq. (4.1.2.6) and $w_{s}{ }^{2}$ replaces $w_{s}$.

The analogous form of Eq. $(4 \cdot 1.2 .12)$ for $M_{2,1}(z, \mu, w)$ after this development is

$$
\mathrm{R}_{z}^{+} \mathrm{M}_{2,1}(\mathrm{z}, \mu, \mathrm{w})=\mathrm{w}_{\mathrm{S}}^{2} \int \mathrm{d} \mu^{-} \mathrm{E}\left(\mathrm{z}, \mu \rightarrow \mu^{-}\right) \mathrm{M}_{2,1}\left(\mathrm{z}, \mu^{-\cdots}, \mathrm{w}\right)+\mathrm{R}_{\mathrm{z}}^{+} \mathrm{S}_{2,1}(\mathrm{z}, \mu, \mathrm{w})
$$

Áīter substituting for $S_{2,1}(z, \mu, w)$ from $E q$. (4.1.2.19) and noting that only the transmission probability factors in $s_{2,1}$ are functions of $z$, Eq. (4.1.2.20) becomes

$$
\begin{aligned}
& \mathrm{R}_{\mathrm{z}}^{+} \mathrm{M}_{2,1}(\mathrm{z}, \mu, \mathrm{w})=\mathrm{w}_{\mathrm{S}}^{2} \int \mathrm{d} \mu{ }^{\prime} \mathrm{E}\left(\mathrm{z}, \mu \rightarrow \mu^{\prime \prime}\right) \mathrm{M}_{2,1}\left(\mathrm{z}, \mu^{\prime}, \mathrm{w}\right) \\
& +H(\mu)\left[\frac{1}{m} M_{2, z_{s}}\left(z_{s}, \mu, w\right)+\left(\frac{m-1}{m}\right)\left\{M_{1, z_{s}}\left(z_{s}, \mu, w\right)\right\}^{2}\right] R_{z}^{+} T\left(z \rightarrow z_{s}, \mu\right) .
\end{aligned}
$$

Applying the identity $(4.1 .2 .4)$ to Eq. $(4 \cdot 1.2 .21)$ :

$$
\begin{aligned}
& \mathrm{R}_{\mathrm{z}}^{+} \mathrm{M}_{2,1}(z, \mu, \mathrm{w})=\mathrm{w}_{\mathrm{S}}^{2} \int \mathrm{d} \mu{ }^{\prime \prime} \mathrm{E}\left(\mathrm{z}, \mu \rightarrow \mu^{\prime \prime}\right) \mathrm{M}_{2,1}\left(\mathrm{z}, \mu^{\prime \prime}, \mathrm{w}\right) \\
& +H(\mu) \delta\left(z-z_{s}\right) \frac{\mu}{\Sigma_{T}}\left[\frac{1}{m} M_{2, z_{s}}\left(z_{s}, \mu, w\right)+\left(\frac{m-1}{m}\right)\left\{M_{1, z_{s}}\left(z_{s}, \mu, w\right)\right\}^{2}\right] .
\end{aligned}
$$


Finally, the definition of the adjoint operater $R_{z}^{+}$is applied to give the integro-differential equation for $M_{2,1}(z, \mu, w)$ in terms of

$$
\begin{aligned}
& F_{2,1}(z, \mu, w) \text { for } 0 \leq z<z_{s}: \\
& -\mu \frac{\partial}{\partial z} F_{2,1}(z, \mu, w)+\Sigma_{T} F_{2,1}(z, \mu, w)=\int d \mu^{\prime \prime} \Sigma_{s}^{*} E\left(z, \mu \rightarrow \mu^{\prime \prime}\right) F_{2,1}\left(z, \mu^{\prime \prime}, w\right) \\
& \quad+H(\mu) \delta\left(z-z_{s}\right) \frac{\mu}{\Sigma_{T}}\left[\frac{1}{m} M_{2, z_{s}}(z, \mu, w)+\left(\frac{m-1}{m}\right)\left\{M_{1, z_{s}}\left(z_{s}, \mu, w\right)\right\}^{2}\right],
\end{aligned}
$$

where

$$
\mathrm{F}_{2,1}(\mathrm{z}, \mu, \mathrm{w}) \equiv \frac{1}{\Sigma_{\mathrm{T}}} \mathrm{M}_{2,1}(\mathrm{z}, \mu, \mathrm{w})
$$

and

$$
\Sigma_{S}^{*} \equiv w_{S}^{2} \Sigma_{T}
$$

In deriving the integro-differential equation for $\mathrm{M}_{2,2}$,

Eqs. (4.1.1.9a and c) are first rewritten for $r=2$ :

$$
\begin{aligned}
& M_{2,2}(z, \mu, w)=w_{s}^{2} \int_{z_{s}<z^{+}<D} d z^{+} \int d^{\prime}-\frac{\dot{\Sigma}_{T}}{|\mu|} T\left(z+z^{+}, \mu\right) E\left(z^{+}, \mu+\mu^{\prime \prime}\right) \\
& \quad \times M_{2,2}\left(z^{+}, \mu^{-\prime}, w\right)+s_{2,2}(z, \mu, w),
\end{aligned}
$$

where

$$
\mathrm{S}_{2,2}(z, \mu, w)=[1-H(\mu)] \cdot T\left(z \rightarrow z_{s}, \mu\right) \cdot m \cdot M_{2, z_{s}}\left(z_{s}, \mu, w\right)+H(\mu) T(z \rightarrow D, \mu) \cdot w^{2} .
$$

Again, the same procedure is followed in transforming Eq. (4.1.2.26) as was followed for $M_{2,1}$, except that $M_{2,2}$ replaces $M_{2,1}$ in the equations 
and all integrations over $\mathrm{dz}^{+}$are restricted to the range of starting locations $z_{s}<z^{+}<D$. The term $S_{2,2}(z, \mu, w)$ also replaces $S_{2,1}(z, \mu, w)$. Thus, an equation analogous to $(4.1 .2 .20)$ is derived for $\mathrm{M}_{2,2}$ and is written (with the terms comprising $s_{2,2}$ explicitiy writean out):

$$
\begin{aligned}
& \mathrm{R}_{z}^{+} \mathrm{M}_{2,2}(z, \mu, w)=\mathrm{w}_{\mathrm{s}}^{2} \int \mathrm{d} \mu^{\prime} \mathrm{E}\left(z, \mu+\mu^{\prime \prime}\right) \mathrm{M}_{2,2}\left(z, \mu^{\prime}, w\right) \\
& \quad+\mathrm{R}_{z}^{+}\left\{[1-\mathrm{H}(\mu)] \mathrm{T}\left(z \rightarrow z_{s}, \mu\right) \cdot \mathrm{m} \cdot \mathrm{H}_{2, z_{s}}\left(z_{s}, \mu, w\right)+H(\mu) \mathrm{T}(z \rightarrow \mathrm{D}, \mu) \cdot \mathrm{w}^{2}\right\}
\end{aligned}
$$

The steps taking EG- $(4.1 .2 .21)$ to Eq, $(4.1 .2 .23)$ artappiad to Eq. (4.1.2.28) in order to derive the integro-differential equation for

$$
\begin{aligned}
& M_{2,2}(z, \mu, w) \text { for } z_{s}<z \leq D: \\
& -\mu \frac{\partial}{\partial z} F_{2,2}(z, \mu, w)+\Sigma_{T} F_{2,2}(z, \mu, w)=\int d \mu-\Sigma_{s}^{*} \cdot E\left(z, \mu \rightarrow \mu^{-\infty}\right) F_{2,2}(z, \mu \cdots, w) \\
& \quad+[1-H(\mu)] \delta\left(z-z_{s}\right) \cdot \frac{|\mu|}{\Sigma_{T}} \cdot m \cdot M_{2, z_{s}}\left(z_{s}, \mu, w\right)+H(\mu) \delta(z-D) \frac{\mu}{\Sigma_{T}} \cdot w^{2},
\end{aligned}
$$

where $\quad F_{2,2}(z, \mu, w) \equiv 1 / \Sigma_{T} M_{2,2}(z, \mu, w)$

and $\Sigma_{S}^{*}$ is defined as in $\mathrm{Eq} \cdot(4 \cdot 1 \cdot 2 \cdot 27)$.

Note that the homogeneous forms of Eqs. (4.1.2.23) and (4.1.2.29) are identical. Hence, one equation which governs the second moment of the slab transmission score for all $z \neq z_{s}$ may be formulated as follows [with $\left.F_{2}(z, \mu, w) \equiv I / \Sigma_{T} \cdot M_{2}(z, \mu, w)\right]:$ 


$$
\begin{aligned}
& -\mu \frac{\partial}{\partial z} F_{2}(z, \mu, w)+\Sigma_{T} F_{2}(z, \mu, w)=\int d \mu-\Sigma_{s}^{*} \cdot E\left(z, \mu \rightarrow \mu^{-\top}\right) F_{2}\left(z, \mu^{-}, w\right) \\
& \quad+H(\mu) \delta(z-D) \frac{\mu}{\Sigma_{T}} \cdot w^{2}, \text { for } z \neq z_{s} .
\end{aligned}
$$

[Note that the term with $\delta(z-D)$ does not in effect add anything to Eq. (4.1.2.23).] Equation (4.1.2.31) is an adjoint formulation of the one-speed Boltzmann transport equatio.. A solution is obtained by solving the homogeneous form of Eq. (4.1.2.31), together with the nonzero boundary condition at $z=D$ and subject to the discontinuities in the solution function at $z=z_{s}$ [characterized in Eqs. (4.1.1.13) and (4.1.i.14)j. The discontinuities are also reflected in the deltafunction source terms at $z=z_{s}$ appearing in the integro-differential equations $[(4 \cdot 1.2 .23)$ and $(4 \cdot 1 \cdot 2.29)]$.

The expected value of the square of the score, given a particle is introduced at $z=D$ with positive direction cosine and $w=1$, is $w^{2}=1$. Thus, the adjoint boundary condition at $z=D$ is written

$$
\begin{aligned}
& M_{2}(D, \mu>0,1)=1 \\
& F_{2}(D, \mu>0, I)=1 / \Sigma_{T} .
\end{aligned}
$$

or

A zero boundary condition exists at $z=0$ :

$$
\begin{aligned}
& M_{2}(0, \mu<0,1)=0 \\
& \text { or } \quad F_{2}(0, \mu<0,1)=0 .
\end{aligned}
$$

Finally, the discontinuity condition in $M_{2}$ at $z=z_{s}$ is rewritten here [from Eqs. (4.1.1.13 and 14)] for $\mu<0$ 


$$
\mathrm{M}_{2}^{-}\left(z_{s}, \mu, w\right)=\frac{1}{m} M_{2}^{+}\left(z_{s}, \mu, w\right)
$$

and for $\mu>0$

$$
M_{2}^{+}\left(z_{s}, \mu, w\right)=m \cdot M_{2}^{-}\left(z_{s}, \mu, w\right)-(m-1)\left\{M_{1, z_{s}}\left(z_{s}, \mu, w\right)\right\}^{2} .
$$

\subsection{Deterministic Prediction of Time per Particle History $\left(\tau_{p}\right)$}

The appropriate equations permitting the prediction of time per particle history $\left(\tau_{p}\right)$ are developed in this section. The development is again based on the transport model presented in Chapter III. The integral first moment equation for $\tau_{p}$ is derived first, followed by a transformation to the appropriate integro-differential form. Most of the nomenclature used here has already been defined at the beginning of Section 4.1; however, additional definitions are made in the course of the development.

\subsubsection{Derivation of First Moment Equation for $\tau_{p}$. As was stated} in Chapter II, the theory of Monte Carlo errors as generalized by Booth and Cashwel1 17 is amenable to the prediction of the computer time required to process a particle history. To do so, the score distribution functions are defined so that the score at each event in the particle's history is the actual computer time required to process that particular event. Thus, the total score in this case represents the total time per particle history. In this context the integral equation for the first moment (expected value) of the time per history may be formulated.

Since the Monte Carlo problem being considered here is such that the weight of the particle in no way influences the time required to 
process it, particle weight does not enter into the final formulations. Thus, the kernels and probability densities can now be defined in terms of the regular, rather than augmented phase-space coordinates, i.e., $\underline{\mathrm{R}}$ and not $\underline{P}$.

The non-weight-dependent kernels and densities are defined below (see also the definitions at the beginning of Section 4.1.1).

$$
\begin{aligned}
& \mathrm{T}\left(\underline{\mathrm{R}}, \underline{\mathrm{R}}^{+}\right) \mathrm{d} \mu^{+} \equiv \int \mathrm{d} w^{+} \mathrm{T}\left(\underline{\mathrm{P}}, \underline{\mathrm{P}}^{+}\right) \mathrm{d} \mu^{+} \\
& =T\left(z \rightarrow z^{+}, \mu\right) \delta\left(\mu^{+}-\mu\right) d \mu^{+} \text {. } \\
& \sigma\left(\underline{R}^{+}, \underline{R}^{\prime}\right) d z^{+}{\underline{d R^{\prime}}}^{\prime} \equiv \int d w^{-} \sigma\left(\underline{P}^{+}, \underline{P}^{\prime}\right) d z^{+} d \underline{R}^{\prime} \\
& =\frac{\Sigma_{T}}{|\mu|} \delta\left(\underline{R}^{-}-\underline{R}^{+}\right) d z^{+} d \underline{R}^{-} . \\
& \mathrm{D}_{0}\left(\underline{\mathrm{R}}, \underline{\mathrm{R}}^{+}\right) \mathrm{dR}^{+} \equiv \int \mathrm{dw} \mathrm{D}_{0}\left(\underline{\mathrm{P}}, \underline{\mathrm{P}}^{+}\right) \mathrm{dR}^{+} \\
& =\left\{\begin{array}{l}
\mathrm{T}\left(z \rightarrow z^{+}, \mu\right)[1-\mathrm{H}(\mu)] \delta\left(\mu^{+}-\mu\right) \delta\left(z^{+}\right) \mathrm{d} z^{+} \mathrm{d}^{+} \text {for } z \leq z_{s}, \\
0 \text { for } z>z_{s} .
\end{array}\right. \\
& \mathrm{D}_{\mathrm{T}}\left(\underline{\mathrm{R}}, \underline{\mathrm{R}}^{+}\right) \mathrm{dR}^{+} \equiv \int \mathrm{dw^{+ }} \mathrm{D}_{\mathrm{T}}\left(\underline{\mathrm{P}}, \underline{\mathrm{P}}^{+}\right) \mathrm{dR}^{+} \\
& =\left\{\begin{array}{l}
T\left(z \rightarrow z^{+}, \mu\right) H(\mu) \delta\left(\mu^{+}-\mu\right) \delta\left(z^{+}-D\right) d z^{+} d \mu^{+} \text {for } z \geq z_{s}, \\
0 \text { for } z<z_{s} .
\end{array}\right.
\end{aligned}
$$




$$
\begin{aligned}
& E\left(\underline{R}^{\prime}, \underline{R}^{\prime \prime}\right) d \underline{R}^{\prime \prime} \equiv \int d w^{\prime} E\left(\underline{P}^{\prime}, \underline{P}^{\prime}\right) d \underline{R}^{\prime} \\
& =E\left(z^{\prime}, \mu^{\prime} \rightarrow \mu^{\prime}\right) \delta\left(z^{-}-z^{\prime}\right) d z^{\prime} d \mu^{\prime} \text {. } \\
& \beta_{0 m}\left(\underline{R}^{+}, \underline{R}^{\prime}\right) d \underline{R}{ }^{\prime} \equiv \int d w^{\prime} \beta_{O m}\left(\underline{P}^{+}, \underline{P}^{\prime}\right) d \underline{R}^{\prime} \\
& =\xi_{\mathrm{m}} \delta\left(\mathrm{z}^{-}-\mathrm{z}^{+}\right) \delta\left(\mu^{-\mu}-\mu^{+}\right) \mathrm{d} \mathrm{z}^{-} \mathrm{d} \mu^{-} \\
& B_{S m}\left(\underline{R}^{+}, \underline{R}^{-\rho}\right) d \underline{R}^{\cdots} \equiv \int d w^{-} B_{S_{m}}\left(\underline{P}^{+}, \underline{P}^{\mu}\right) \underline{d R}^{-} \\
& =\delta\left(z^{-}-z^{+}\right) \delta\left(\mu^{-\infty}-\mu^{+}\right) d z^{-} \mathrm{d} \mu^{-\infty} .
\end{aligned}
$$

Since the score distribution functions for the expected time problem do not have any weight dependence, we can write

$$
p_{i}\left(\underline{R}^{\prime}, s\right) d s \equiv p_{i}\left(\underline{P}^{\prime}, s\right) d s,
$$

where the index $i$ refiers to any specific event category. Also for the same reason

$$
\begin{aligned}
& S_{m}\left(\underline{R}^{+}\right) d z^{+} \equiv S_{m}\left(\underline{P}^{+}\right) d z^{+}, \\
& R_{m}\left(\underline{R}^{+}\right) d z^{+} \equiv R_{m}\left(\underline{P}^{+}\right) d z^{+},
\end{aligned}
$$

The development proceeds by first writing directly the first moment equations $(r=1)$ from Appendix $B$, since the equation for the expected value of time per particle history may be formally expressed up to this point as any other first moment formulation. We consider in detail the case for $z$ restricted to the range $z_{s}<z \leq D$. Equations (B.1.12) and (B.1.22) are rewritten here as 


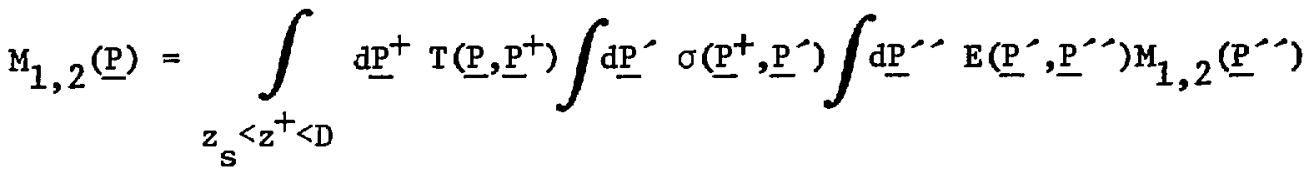

$$
\begin{aligned}
& +\int \underline{\mathrm{P}}^{+} \mathrm{T}\left(\underline{\underline{p}}, \underline{\mathrm{P}}^{+}\right) \mathrm{R}_{\mathrm{m}}\left(\underline{\mathrm{P}}^{+}\right) \int \underline{\mathrm{P}}^{-} \mathrm{B}_{0 \mathrm{~m}}\left(\xi_{\mathrm{m}} ; \underline{\mathrm{P}}^{+}, \underline{\mathrm{P}}^{-}\right) \mathrm{M}_{1, \mathrm{~s}}\left(\underline{\mathrm{P}}^{-}\right)+\mathrm{Q}_{1,2}(\underline{\mathrm{P}}) \text {, }
\end{aligned}
$$

where

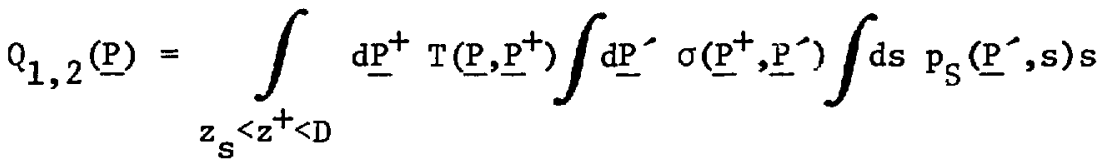

$$
\begin{aligned}
& +\int \mathrm{dP}^{+} \mathrm{D}_{\mathrm{T}}\left(\underline{\mathrm{P}}, \underline{\mathrm{P}}^{+}\right) \int \mathrm{d} s \mathrm{p}_{\mathrm{E}, \mathrm{D}}\left(\underline{\mathrm{P}}^{+}, \mathrm{s}\right) \mathrm{s}+\int \mathrm{d \underline {P } ^ { + }} \mathrm{T}\left(\underline{\mathrm{P}}, \underline{\mathrm{P}}^{+}\right) \mathrm{R}_{\mathrm{m}}\left(\underline{\mathrm{P}}^{+}\right) \int \mathrm{ds} \\
& \times p_{R}\left(\underline{p}^{+}, s\right) s \text {. }
\end{aligned}
$$

The integral over $\mathrm{dP}^{\prime \prime}$ in the second term of Eq. (4.2.1.1) may be simplified by recalling the definition of $\mathrm{B}_{0 \mathrm{~m}}$ (from beginning of Chapter IV) and integrating:

$$
\begin{aligned}
& \int \underline{\mathrm{P}}^{\prime \prime} \mathrm{B}_{0 \mathrm{~m}}\left(\xi_{\mathrm{m}} ; \underline{\mathrm{P}}^{+}, \underline{\mathrm{P}}^{\prime \prime}\right) \mathrm{M}_{1, \mathrm{z}_{\mathrm{s}}}\left(\underline{\mathrm{P}}^{\prime \prime}\right)=\int \mathrm{dR}^{\prime} \int \mathrm{dw}^{\prime \prime} \mathrm{B}_{0 \mathrm{~m}}\left(\underline{\mathrm{P}}^{+}, \underline{\mathrm{P}}^{\prime \prime}\right) \mathrm{M}_{1, \mathrm{z}_{\mathrm{s}}}\left(\underline{\mathrm{P}}^{\prime \prime}\right) \\
& \left.+\int \mathrm{dR}^{\prime} \int \mathrm{d} \mathrm{w}^{\prime} \delta\left(\mathrm{z}^{\prime}-\mathrm{z}^{+}\right) \delta\left(\mu^{\prime}-\mu^{+}\right)\left(1-\xi_{\mathrm{m}}\right) \delta\left(\mathrm{w}^{\prime}\right) \mathrm{M}_{1, \mathrm{z}_{\mathrm{s}}} \underline{\mathrm{R}}^{\prime}, \mathrm{w}^{\prime}\right) \text {. }
\end{aligned}
$$

However, weight zero implies a terminated particle; thus

$$
\left.\int \delta\left(w^{\prime}\right) M_{1, z_{s}}\left(\underline{R}^{-}, w^{-\prime}\right) d w^{-}=M_{1, z_{s}} \underline{(R}^{-}, 0\right) \equiv 0
$$

by the identity $(4.1 .1 .6)$, and hence 


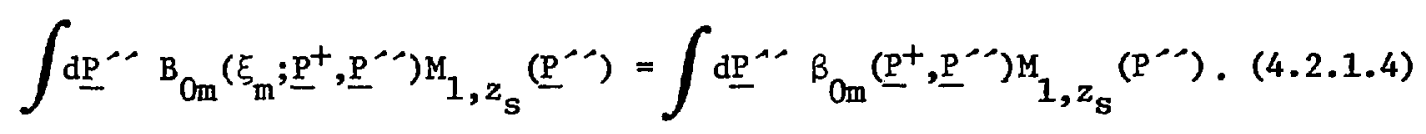

For notational purposes, the first moment of the time per particle history will be represented as

$$
M_{1,2}(\underline{P}) \equiv \tau_{p, \dot{2}^{(P)}} \text {. }
$$

Also, since the weight of a particle has no bearing on the scores and, hence, on the expected value of $\tau_{p}$ '

$$
\tau_{p, 2} \stackrel{(P)}{\equiv} \tau_{p, 2}(\underline{R}, w)=\tau_{p, 2} \underline{(R)} \text {. }
$$

The implicit integrations over weight are performed in Eqs. (4.2.1.1) and (4.2.1.2). The definitions at the beginning of this section, as we11 as Eqs. $(4.2 .1 .4),(4.2 .1 .5)$, and $(4.2 .1 .6)$ are employed to write (for $\underline{R}$ with $\left.z_{s}<z \leq D\right)$ ):

$$
\begin{aligned}
& \tau_{p, 2}(\underline{R})=\int_{z_{s}<z^{+}<\mathrm{D}} \underline{\mathrm{R}}^{+} \mathrm{T}\left(\underline{\mathrm{R}}, \underline{\mathrm{R}}^{+}\right) \int \underline{\mathrm{d}}^{\prime} \sigma\left(\underline{\mathrm{R}}^{+}, \underline{\mathrm{R}}^{\prime}\right) \int \mathrm{dR}^{\prime \prime} \mathrm{E}\left(\underline{\mathrm{R}}^{\prime}, \underline{\mathrm{R}}^{\prime \prime}\right) \tau, \underline{\mathrm{R}}_{\mathrm{p}}\left(\underline{\mathrm{R}}^{\prime \prime}\right) \\
& +\int \mathrm{dR}^{+} \mathrm{T}\left(\underline{\mathrm{R}}, \underline{\mathrm{R}}^{+}\right) \mathrm{R}_{\mathrm{m}}\left(\underline{\mathrm{R}}^{+}\right) \int \mathrm{d \textrm {R } ^ { \prime }} \beta_{0 \mathrm{~m}}\left(\underline{\mathrm{R}}^{+}, \underline{\mathrm{R}}^{\prime}\right) \tau_{\mathrm{p}, \mathrm{z}_{\mathrm{s}}}\left(\underline{\mathrm{R}}^{\prime}\right)+Q_{\mathrm{p}, 2}(\underline{\mathrm{R}}),
\end{aligned}
$$

where

$$
\begin{aligned}
& Q_{p, 2}(\underline{R})=\int_{\substack{z_{s}<z^{+}<D}} d \underline{R}^{+} T\left(\underline{R}, \underline{R}^{+}\right) \int \underline{R}^{\prime} \sigma\left(\underline{R}^{+}, \underline{R}^{\prime}\right) \int d s p_{S}\left(\underline{R}^{\prime}, s\right) s \\
& +\int \mathrm{dR}^{+} \mathrm{D}_{\mathrm{T}}\left(\underline{\mathrm{R}}, \underline{\mathrm{R}}^{+}\right) \int \mathrm{d} s \mathrm{P}_{\mathrm{E}, \mathrm{D}}\left(\underline{\mathrm{R}}^{+}, s\right) s+\int \mathrm{d \underline {R } ^ { + }} T\left(\underline{\mathrm{R}}, \underline{\mathrm{R}}^{+}\right) \mathrm{R}_{\mathrm{m}}\left(\underline{\mathrm{R}}^{+}\right) \int \mathrm{d} s \\
& \times \mathrm{p}_{\mathrm{R}}\left(\mathrm{R}^{+}, \mathrm{s}\right) \mathrm{s} \text {. }
\end{aligned}
$$


In Eqs. (4.2.1.7a, b), the definitions stated in Eqs. $(4.2 .1 .5,6)$ were extended in writing $\tau_{p, z_{s}}\left(\underline{R}^{\prime \prime}\right)=\tau_{p, z_{s}}\left(P^{\prime \prime}\right) \equiv M_{1, z_{s}}\left(\underline{P}^{\prime \prime}\right)$. Also, $Q_{p, 2}(\underline{R})=Q_{p, 2}(\underline{P}) \equiv Q_{1,2}(\underline{P})$, since there is no weight dependence in the source terms.

Given the additional definitions

$$
\begin{aligned}
& \tau_{p, 1} \underline{(R)}=\tau_{p, 1}(\underline{P}) \equiv M_{1,1} \underline{(P)}, \\
& Q_{p, 1} \underline{(R)}=Q_{p, 1} \underline{(P)} \equiv Q_{1,1} \underline{(P)},
\end{aligned}
$$

and

$$
Q_{p, z_{s}}(\underline{R})=Q_{p, z_{s}}(\underline{P}) \equiv Q_{1, z_{s}}(\underline{P}) \text {, }
$$

it is possible to apply the same procedure as detailed above to Eqs. (B.1.11), (B.1.20), (B.1.13), and (B.1.24) to yield the proper formulations for $\tau_{p, 1}(\underline{R})$ and $\tau_{p, z_{s}}(\underline{R})$. The resultant equations are written:

$$
\begin{aligned}
& \tau_{\mathrm{p}, 1}(\underline{\mathrm{R}})=\int_{0<\mathrm{z}^{+}<\mathrm{z}_{s}} \mathrm{d \textrm {R } ^ { + }} \mathrm{T}\left(\underline{\mathrm{R}}, \underline{\mathrm{R}}^{+}\right) \int \mathrm{d}^{\prime} \sigma\left(\underline{\mathrm{R}}^{+}, \underline{\mathrm{R}}^{\prime} ; r_{\mathrm{d} \underline{R}^{\prime \prime}} E\left(\underline{\mathrm{R}}^{\prime}, \underline{\mathrm{R}}^{\prime \prime}\right) \tau_{\mathrm{p}, 1}\left(\underline{\mathrm{R}}^{\prime \prime}\right)\right. \\
& +\int \mathrm{dR}^{+} \mathrm{T}\left(\underline{\mathrm{R}}, \underline{\mathrm{R}}^{+}\right) \mathrm{S}_{\mathrm{m}}\left(\underline{\mathrm{R}}^{+}\right) \int \mathrm{d}^{\prime}{ }^{\prime} \mathrm{B}_{\mathrm{Sm}}\left(\underline{R}^{+}, \underline{\mathrm{R}}^{\prime \prime}\right) \cdot \mathrm{m} \cdot \tau_{\mathrm{p}, \mathrm{z}_{\mathrm{s}}}\left(\underline{\mathrm{R}}^{\prime \prime}\right)+\mathrm{Q}_{\mathrm{p}, 1} \underline{(\underline{R})},
\end{aligned}
$$

where

$$
\begin{aligned}
& Q_{p, 1}(\underline{R})=\int_{0<z^{+}<z_{s}} d \underline{R}^{+} T\left(\underline{R}, \underline{R}^{+}\right) \int d_{\underline{R}}^{-} \sigma\left(\underline{R}^{+}, \underline{R}^{-}\right) \int d s P_{S}\left(\underline{R}^{-}, s\right) s+\int d \underline{R}^{+} \\
& \times D_{0}\left(\underline{R}, \underline{R}^{+}\right) \int d s P_{E, 0}\left(\underline{R}^{+}, s\right) s+\int d R^{+} T\left(\underline{R}, \underline{R}^{+}\right) S_{m}\left(\underline{R}^{+}\right) \int d s P_{S m}\left(\underline{R}^{+}, s\right) s
\end{aligned}
$$


for $\underline{R}$ with $0 \leq z<z_{s}$, and

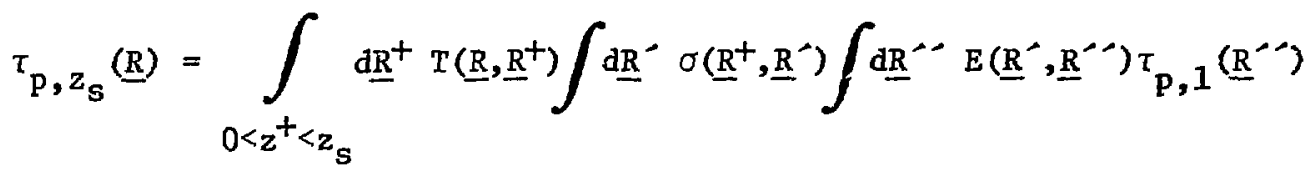

$$
\begin{aligned}
& \left.+\int_{z_{s}<z^{+}<D} \underline{\mathrm{R}}^{+} \mathrm{T}\left(\underline{\mathrm{R}}, \underline{\mathrm{R}}^{+}\right) \int \mathrm{dR}^{\prime} \sigma\left(\underline{\mathrm{R}}^{+}, \underline{\mathrm{R}}^{\prime}\right) \int \underline{\mathrm{R}}^{-1} \mathrm{E}\left(\underline{\mathrm{R}}^{\prime}, \underline{\mathrm{R}}^{\prime \prime}\right) \tau_{\mathrm{p}, 2}{\underline{\left(\mathrm{R}^{\prime}\right.}}^{\prime}\right) \\
& +Q_{p, z_{s}}(\underline{R})
\end{aligned}
$$

where

$$
\begin{aligned}
& \mathrm{Q}_{\mathrm{p}, z_{\mathrm{s}}}(\underline{R})=\int_{0<\mathrm{z}^{+}<\mathrm{z}_{\mathrm{S}}} \underline{\mathrm{R}}^{+} \mathrm{T}\left(\underline{R},{\underline{R^{+}}}^{+}\right) \int \underline{\mathrm{R}}^{*} \sigma\left(\underline{R}^{+}, \underline{\underline{R}}^{\prime}\right) \int \mathrm{ds} \mathrm{p}_{\mathrm{S}}\left(\underline{R}^{\prime}, s\right) s \\
& +\int_{z_{s}<z^{+}<D} \underline{\mathrm{R}}^{+} \mathrm{T}\left(\underline{\mathrm{R}}, \underline{\mathrm{R}}^{+}\right) \int \underline{\mathrm{dR}}^{-} \sigma\left(\underline{\mathrm{R}}^{+}, \underline{\mathrm{R}}^{-}\right) \int \mathrm{ds} \mathrm{p}_{\mathrm{S}}\left(\underline{\mathrm{R}}^{-}, \mathrm{s}\right) \mathrm{s} \\
& \left.+\int \mathrm{dR}^{+} \mathrm{D}_{0}\left(\underline{\mathrm{R}}, \underline{\mathrm{R}}^{+}\right) \int \mathrm{d} s \mathrm{p}_{\mathrm{E}, 0}\left(\underline{\mathrm{R}}^{+}, \mathrm{s}\right) \mathrm{s}+\int \mathrm{dR}^{+} \mathrm{D}_{\mathrm{T}}\left(\underline{\mathrm{R}}, \underline{\mathrm{R}}^{+}\right) \int \mathrm{ds} \mathrm{p}_{\mathrm{E}, \mathrm{D}} \underline{(\underline{R}}^{+}, s\right) \mathrm{s}
\end{aligned}
$$

for $\underline{R}$ with $z=z_{s}$.

The coupled Eqs. $(4 \cdot 2.1 .9 \mathrm{a}, \mathrm{b}),(4.2 .1 .10 \mathrm{a}, \mathrm{b})$, and $(4.2 .1 .7 \mathrm{a}, \mathrm{b})$ formulate the time per particle history over the entire range of starting positions $z$ : 


$$
\tau_{p}(z, \mu)= \begin{cases}\tau_{p, 1}(z, \mu) & \text { for } 0 \leq z<z_{s}, \\ \tau_{p, z_{s}}\left(z_{s}, \mu\right) & \text { for } z=z_{s}, \text { and } \\ \tau_{p, 2}(z, \mu) & \text { for } z_{s}<z \leq D,\end{cases}
$$

where $\tau_{p}(z, \mu)$ represents the expected computer time required to process the remaining particle history, given the particle begins (or resumes) its flight with coordinates $\underline{R}=(z, \mu)$.

The details for further development of the $\tau_{p}$ equations appear in Appendix C.

The first moment integral equations for the expected time per history are rewritten here from Appendix C [Eqs. (C.1.2), (C.1.3), (C.1.4)]. For particle starting locations restricted to the range $0 \leq \mathrm{z}<\mathrm{z}_{\mathrm{s}}$

$$
\begin{aligned}
& \tau_{p, 1}(z, \mu)=\int_{0<z^{+}<z_{s}} d z^{+} \int \mathrm{d} \mu^{-} \mathrm{k}\left(z, \mu \rightarrow z^{+}, \mu^{-}\right) \tau_{p, 1}\left(z^{+}, \mu^{-\infty}\right) \\
& +H(\mu) \cdot m \cdot T\left(z \rightarrow z_{s}, \mu\right) \tau_{p, z_{s}}\left(z_{s}, \mu\right)+\int_{0<z^{+}<z_{s}} d z+\frac{\Sigma_{T}}{|\mu|} T\left(z \rightarrow z^{+}, \mu\right) \cdot \tau_{c} \\
& +[1-H(\mu)] \cdot T(z \rightarrow 0, \mu) \cdot \tau_{0}+H(\mu) T\left(z \rightarrow z_{s}, \mu\right) \cdot \tau_{s} .
\end{aligned}
$$

For particles starting with $\mathrm{z}$ in the range $\mathrm{z}_{\mathrm{s}}<\mathrm{z} \leq \mathrm{D}$ : 


$$
\begin{aligned}
& \tau_{p, 2}(z, \mu)=\int_{z_{s}<z^{+}<D} d z^{+} \int d \mu^{-\infty} k\left(z, \mu \rightarrow z^{+}, \mu^{-\prime}\right) \tau_{p, 2}\left(z^{+}, \mu^{-\infty}\right) \\
& +[1-H(\mu)] \cdot \frac{1}{m} \cdot T\left(z \rightarrow z_{s}, \mu\right) \tau_{p, z_{s}}\left(z_{s}, \mu\right)+\int_{z_{s}<z^{+}<D} d z^{+} \frac{\Sigma T}{|\mu|} \\
& x T\left(z \rightarrow z^{+}, \mu\right) \cdot \tau_{c}+H(\mu) T(z \rightarrow D, \mu) \cdot \tau_{T}+[1-H(\mu)] T\left(z \rightarrow z_{s}, \mu\right) \cdot \tau_{R} .
\end{aligned}
$$

Fina1ly, for particles starting at the splitting surface $\left(z=z_{s}\right)$ :

$$
\begin{aligned}
& \tau_{p, z_{s}}\left(z_{s}, \mu\right)=\int_{0<z^{+<z_{s}}} d z \int d \mu^{\cdots} k\left(z_{s}, \mu \rightarrow z^{+}, \mu^{\cdots}\right) \tau_{p, 1}\left(z^{+}, \mu^{-\infty}\right) \\
& \left.+\int_{z_{s}<z^{+}<D} d z^{+} \int d \mu^{\cdots} k\left(z, s^{\prime} \mu z^{+}, \mu^{\cdots}\right) \tau, p, z^{+}, \mu^{\prime \prime}\right)+\int_{0<z^{+}<z_{s}} d z^{+} \\
& \times \frac{\Sigma_{T}}{|\mu|} T\left(z_{s} \rightarrow z^{+}, \mu\right) \cdot \tau_{c}+\int_{z_{s}<z^{+}<D} d z^{+} \frac{\Sigma_{T}}{|\mu|} T\left(z_{s} \rightarrow z^{+}, \mu\right) \cdot \tau_{c}+[1-H(\mu)] \\
& x \mathrm{~T}\left(z_{S} \rightarrow 0, \mu\right) \cdot \tau_{0}+H(\mu) \mathrm{T}\left(z_{S} \rightarrow D, \mu\right) \cdot \tau_{T} \text {. }
\end{aligned}
$$

In Eqs. $(4.2 .1 .12-14) \tau_{c}, \tau_{s}, \tau_{\mathrm{B}}, \tau_{0}, \tau_{T}$ represent the actual computer times required to process a collision, splitting event, Russian roulette event, front-surface escape, and tally-surface escape (respectively). Also, as shown in Appendix $C$, a discontinuity exists in the solution function for $\tau_{p}$ at the splitting surface. This discontinuity is characterized: 
for $\mu>0$,

$$
\tau_{p}^{-}\left(z_{s}, \mu\right)=m \cdot \tau_{p}^{+}\left(z_{s}, \mu\right)+\tau_{s},
$$

and for $\mu<0$,

$$
\tau_{p}^{+}\left(z_{s}, \mu\right)=\frac{1}{m} \cdot \tau_{p}{ }^{-} \cdot\left(z_{s}, \mu\right)+\tau_{R} .
$$

The definitions for $\tau_{p}^{-}$and $\tau_{p}^{+}$are stated as

$$
\tau_{p}^{-}\left(z_{s}, \mu\right) \equiv \lim _{z \rightarrow z_{s-}} \tau_{p, 1}(z, \mu)
$$

and

$$
\tau_{p}^{+}\left(z_{s}, \mu\right) \equiv \lim _{z \rightarrow z_{s+}} \tau_{p, 2}(z, \mu)
$$

As in the case of the second moment of the slab transmission score, the discontinuities at $z=z_{s}$ are intuitively plausible. For a particle about to undergo splitting $(\mu>0)$, the expected time jumps by a factor equal to the number of sub-particles produced at the splitting event and the computer time required to process the splitting is added. Also, since only $1 / \mathrm{m}$ of the particles coming into a Russian roulette event $(\mu<0)$ survive, the expected time drops accordingly and the time required to process the event itself is added.

\subsubsection{Conversion to Integro-differential Form. Equations (4.2.1.12)} and (4.2.1.13) may be converted to an integro-differential form by following the same formalism as demonstrated in Section 4.1 .2 for the $\mathrm{r}-\mathrm{th}$ moment of the transmission score. 
For particle starting locations in the range $0 \leq z<z_{s}$,

Eq. (4.2.1.12) may be written in the following form [employing identity $(4.1 .1 .12)]:$

$$
\begin{aligned}
\tau_{p, 1}(z, \mu) & =\int_{0<z^{+}<z_{s}} d z^{+} \int d \mu^{-} \frac{\Sigma_{T}}{|\mu|} T\left(z+z^{+}, \mu\right) E\left(z^{+}, \mu+\mu^{\prime \prime}\right) \tau_{p, 1}\left(z^{+}, \mu^{\prime \prime}\right) \\
& +\int_{0<z^{+}<z_{s}} d z^{+} \frac{\Sigma_{T}}{|\mu|} T\left(z \rightarrow z^{+}, \mu\right) \cdot \tau_{c}+S_{p, 1}(z, \mu)
\end{aligned}
$$

where

$$
\begin{aligned}
\mathrm{S}_{\mathrm{p}, 1}(z, \mu) & =\mathrm{H}(\mu) \mathrm{T}\left(z \rightarrow z_{s^{s}} \mu\right)\left[\mathrm{m} \cdot \tau_{\mathrm{p}, z_{\mathrm{s}}}\left(z_{s}, \mu\right)+\tau_{s}\right]+[1-H(\mu)] \\
& \times \mathrm{T}(z \rightarrow 0, \mu) \cdot \tau_{0} .
\end{aligned}
$$

With the definition

$$
\mathrm{G}^{+}\left(\mathrm{z}^{+}, \mu\right) \equiv \int \mathrm{d} \mu^{\infty} \mathrm{E}\left(\mathrm{z}^{+}, \mu \rightarrow \mu^{-\infty}\right) \tau_{\mathrm{p}, 1}\left(\mathrm{z}^{+}, \mu^{-\infty}\right)+\tau_{\mathrm{c}},
$$

it is possible to rewrite Eq. $(4 \cdot 2 \cdot 2.1 \mathrm{a})$ as

$$
\tau_{p, 1}(z, \mu)=\int_{0<z^{+}<z_{s}} d z^{+} \frac{\Sigma_{T}}{|\mu|} T\left(z \rightarrow z^{+}, \mu\right) G^{+}\left(z^{+}, \mu\right)+s_{p, 1}(z, \mu) \cdot(4.2 .2 .3)
$$

However, since $0<z<z_{s}$,

$$
G^{+}(z, \mu)=\int_{0<z^{+}<z s} \delta\left(z-z^{+}\right) G^{+}\left(z^{+}, \mu\right) d z^{+}
$$


Thus by employing identity (4.1.2.1),

$$
\mathrm{G}^{+}(\mathrm{z}, \mu)=\mathrm{R}_{\mathrm{z}}^{+} \int_{0<z^{+}<\mathrm{z}_{\mathrm{s}}} \mathrm{C}\left(\mathrm{z} \rightarrow \mathrm{z}^{+}, \mu\right) \mathrm{G}^{+}\left(\mathrm{z}^{+}, \mu\right) \mathrm{d} \mathrm{z}^{+},
$$

noting that $R_{z}^{+}$is independent of $z^{+}$. Substitution of the definition stated in Eq. (4.1.2.3) into Eq. (4.2.2.3) and rearrangement yields

$$
\int_{0<z^{+}<z_{s}} d z^{+} c\left(z+z^{+}, \mu\right) G^{+}\left(z^{+}, \mu\right)=\tau_{p, 1}(z, \mu)-s_{p, 1}(z, \mu) .
$$

The operator $\mathrm{R}_{\mathrm{z}}^{+}$is then applied to the preceeding equation and Eq. (4.2.2.5) is used to write:

$$
G^{+}(z, \mu)=R_{z}^{+}\left[\tau_{p, 1}(z, \mu)-S_{p, 1}(z, \mu)\right] .
$$

Applying the definition of $\mathrm{G}^{+}(\mathrm{z}, \mu, \mathrm{w})$ as stated in Eq. (4.2.2.2), the above equation is written (after rearranging):

$R_{z}^{+} \tau_{p, 1}(z, \mu)=\int d \mu^{\cdots} E\left(z, \mu \rightarrow \mu^{\prime \prime}\right) \tau_{p, 1}\left(z, \mu^{\cdots}\right)+\tau_{c}+R_{z}^{+} S_{p, 1}(z,$,

Finally, substituting for $s_{p, 1}(z, \mu)$ from Eq. (4.2.2.1b), employing the identity (4.1.2.4), and explicitly writing the transport operator $\mathrm{R}_{\mathrm{z}}^{+}$, Eq. (4:2.2.8) becomes

$$
\begin{aligned}
& -\mu \frac{\partial}{\partial z} F_{T, 1}(z, \mu)+\Sigma_{T} F_{T, 1}(z, \mu)=\int \mathrm{d} \mu \cdots \Sigma_{T} E\left(z, \mu \rightarrow \mu^{\cdots \rho}\right) F_{T, 1}\left(z, \mu^{\prime \prime}\right)+T_{c} \\
& \quad+H(\mu) \delta\left(z-z_{s}\right) \frac{\mu}{\Sigma_{T}}\left[m^{*} \tau_{p, z_{s}}\left(z_{s}, \mu\right)+\tau_{s}\right]+[1-H(\mu)] \delta(z-0) \frac{|\mu|}{\Sigma_{T}} \cdot \tau_{0},
\end{aligned}
$$


where

$$
\mathrm{F}_{\mathrm{T}, 1}(z, \mu) \equiv I / \Sigma_{\mathrm{T}} \cdot \tau_{\mathrm{p}, 1}(z, \mu)
$$

A similar transformation may be performed for $\tau_{p, 2}(z, \mu)$. Equation (4.2.1.13) may be rewritten here as.

$$
\begin{aligned}
& \tau_{p, 2}(z, \mu)=\int_{z_{s}<z^{+}<D} \mathrm{dz}^{+} \int \mathrm{d} \mu^{-} \frac{\Sigma_{\mathrm{T}}}{|\mu|} \mathrm{T}\left(\mathrm{z}+\mathrm{z}^{+}, \mu\right) \mathrm{E}\left(\mathrm{z}^{+}, \mu+\mu^{\prime}\right) \tau_{\mathrm{p}, 2^{2}}\left(\mathrm{z}^{+}, \mu^{-\prime}\right) \\
& +\int_{z_{s}<z^{+}<D} \mathrm{~d} z^{+} \frac{\Sigma_{\mathrm{T}}}{|\mu|} \mathrm{T}\left(z \rightarrow z^{+}, \mu\right) \cdot \tau_{c}+\mathrm{S}_{p, 2}(z, \mu),
\end{aligned}
$$

where

$$
\begin{aligned}
S_{p, 2}(z, \mu) & =[1-H(\mu)] T\left(z+z_{s}, \mu\right)\left[\frac{1}{m} \cdot \tau_{p, z_{S}}\left(z_{s}, \mu\right)+\tau_{R}\right] \\
& +H(\mu) \mathrm{T}(z+D, \mu) \cdot \tau_{T} .
\end{aligned}
$$

The same procedure may be followed in transforming Eq. (4.2.2.10a) as was employed for $\tau_{p, 1}$. Now, however, $\tau_{F, 2}$ replaces $\tau_{p, 1}$ in the equations and all indicated integrations over $\mathrm{dz}^{+}$are restricted to the range $z_{s}<z^{+}<D$. The term $S_{p, 2}$ aìso replaces $S_{p, 1^{*}}$ Thus, by following steps analogous to those encompassing Eqs. (4.2.2.2) through (4.2.2.9), Eqs. (4.2.2.10) may be transformed to the integro-differential form: 


$$
\begin{aligned}
& -\mu \frac{\partial}{\partial z} F_{T, 2}(z, \mu)+\Sigma_{T_{T} F}(z, \mu)=\int \mathrm{d} \mu^{-}-\Sigma_{T} E\left(z, \mu \rightarrow \mu^{-\prime}\right) F_{T, 2}\left(z, \mu^{-\prime}\right)+\tau_{c} \\
& +[1-H(\mu)] \delta\left(z-z_{s}\right) \frac{|\mu|}{\Sigma_{T}}\left[\frac{1}{m} \cdot \tau_{p}\left(z_{s}, \mu\right)+\tau_{R}\right] \\
& +H(\mu) \delta\left(z-\text { D) } \frac{\mu}{\Sigma_{T}} \cdot \tau_{T},\right.
\end{aligned}
$$

where

$$
F_{T, 2}(z, \mu) \equiv 1 / \Sigma_{T} \cdot \tau_{p, 2}(z, \mu)
$$

Thus, the conversion to integro-differential form results in two equations [Eqs. $(4 \cdot 2,2.9)$ and $(4 \cdot 2.2 .11)$ ], the homogeneous forms of which are identical. In fact, since the surface source term at $z=D$ vanishes in region 1 and the surface source at $z=0$ vanishes in region 2, it is possible to formulate one equation for $\tau_{p}(z, \mu)$ [rather, for $\left.F_{T}(z, \mu) \equiv 1 / \Sigma_{T} \cdot \tau_{p}(z, \mu)\right]$ for all $z \neq z_{S}$ as follows:

$$
\begin{aligned}
& -\mu \frac{\partial}{\partial z} F_{T}(z, \mu)+\Sigma_{T} F_{T}(z, \mu)=\int d \mu^{-} \Sigma_{T} E\left(z, \mu \rightarrow \mu^{-\rho}\right) F_{T}(z, \mu-\rho)+\tau_{c} \\
& \quad+[1-H(\mu)] \delta(z-0) \frac{|\mu|}{\Sigma_{T}} \cdot \tau_{0}+H(\mu) \delta(z-D) \frac{\mu}{\Sigma_{T}} \cdot \tau_{T} \cdot
\end{aligned}
$$

The non-zero adjoint boundary conditions implied by Eq. (4.2.2.12) may be written as follows:

at $z=D$ and $\mu>0$, 


$$
\begin{gathered}
\tau_{\mathrm{p}}(D, \mu>0)=\tau_{\mathrm{T}} \\
\text { or } \mathrm{F}_{\mathrm{T}}(D, \mu>0)=\tau_{\mathrm{T}} / \Sigma_{\mathrm{T}}, \\
\text { and at } z=0 \text { and } \mu<0, \\
\tau_{\mathrm{p}}(0, \mu<0)=\tau_{0} \\
\text { or } \quad \mathrm{F}_{\mathrm{T}}(0, \mu<0)=\tau_{0} / \Sigma_{\mathrm{T}} .
\end{gathered}
$$

Also, the solution of Eq. $(4.2 .2 .12)$ is subject to the discontinuity conditions characterized in Eqs. $(4.2 .1 .15)$ and $(4.2 .1 .16)$ and reflected in the delta-function source terms at $z=z_{s}$ appearing in Eqs. (4.2.2.9) and (4.2.2.11). The jump conditions are rewritten here: for $\underline{\mu>0}$,

$$
\tau_{p}-\left(z_{s}, \mu\right)=m \cdot \tau_{p}^{+}\left(z_{s}, \mu\right)+\tau_{s}
$$

and for $\underline{\mu<0}$,

$$
\tau_{p}^{+}\left(z_{s}, \mu\right)=\frac{1}{m} \cdot \tau_{p}^{-}\left(z_{s}, \mu\right)+\tau_{R}
$$

4.3 Solution to the Equations by Employing Discrete Ordinates $\left(\mathrm{S}_{n}\right)$ Technique

The particular numerical technique used to solve for the first two moments of the transmission score as well as the expected value of time per particle history (by solving the integro-differential equations for $F_{1}^{*}, F_{2}$, and $F_{T}$ respectively) is discussed here. The ONETRAN $S_{n}$ code $i s$ described very briefly, and the required modifications to the program are presented. 


\subsubsection{Equations as Adjoint Formulations of the Boltzmann Trans-}

port Equation. The integro-differential equations for the first and second moments of the slab transmission score (as developed in Section 4.1.2) are actually adjoint formulations of the one-speed Boltzmann transport equation. Thus, the equations are amenable to a standard numerical solution technique commonly employed in the deterministic solution of radiation transport problems--namely, a discrete ordinates $\left(S_{n}\right)$ solution. The same is true of the equation governing time per particle history.

Specifically, the homogeneous forms of Eqs. (4.1.2.14) and (4.1.2.31) are solved using $S_{n}$ for the first and second moments of the transmission score (respectively). The solutions are subject to the non-zero boundary conditions (effectively acting as adjoint boundary sources) at the tally surfare $z=D$ [see Eqs. $(4.1 .2 .16)$ and $(4.1 .2 .32)$ ]. A one-speed calculation is considered. As was alluded to earlier in the development, all evidence of the variance reduction techniques is missing from the formulation of the first moment (expected value) of the transmission score. Hence, the first moment solution is simply the regular adjoint solution to the particle transport problem. On the other hand, survival biasing affects the second moment solution by introducing a "fictitious" scattering cross section $\left(\Sigma_{S}^{*}=w_{s}^{2} \Sigma_{T}\right)$. Moreover, the consideration of splitting and Russian roulette imposes a discontinuity in the second moment solution at the splitting surface $z=z_{s}$. This is characterized in Eqs. (4.1.2.34) and (4.1.2.35). It should be noted that the jump conditions for the second moment require 
prior solution to the first moment equation. Accordingly, the two moments of the transmission score are solved recursively.

An $S_{n}$ technique is also employed to solve $\mathrm{Eq} \cdot(4.2 .2 .12)$ for $\tau_{p}(z, \mu)$ [actually by solving for $F_{T}(z, \mu)$ ]. Non-zero adjoint boundary conditions at $z=0$ and $z=D$ again effectively represent boundary sources [see Eqs. $(4.2 .2 .13)$ and $(4.2 .2 .14)]$. The solutio. for $T_{p}$ also includes treatment of an isotropic distributed source $\tau_{c}$. As expressed in Eqs. $(4.2 .2 .15)$ and $(4.2 .2 .16)$, a discontinuity at $z_{s}$ is imposed on the solution due to splitting and Russian roulette. Finally, the transport problem which is actually solved in solving for the time per particle history ręlects a "fictitious" purely-scattering medium (effectively $\Sigma_{S}=\Sigma_{T}$ ). This results from the implementation of survival biasing in the Monte Carlo problem; thus, particle absorption in the medium is treated by weight reduction rather than real termination of the particle track. As far as time per particle history is concerned, the scattering rat' $\supset\left(\Sigma_{S} / \Sigma_{T}\right)$ at each collision site is irrelevant. All collisions take the. ne amount of time, so that the medium "appears" to be purely scattering.

\subsubsection{The ONETRAN $\left(S_{n}\right)$ Radiation Transport Code. The ONETRAN} $\operatorname{code}^{21}$ developed at the Los Alamos Scientific Laboratory was used in this work to solve deterministically for the first two moments of the transmission score. This allowed for the prediction of the (true) sample variance of a corresponding Monte Carlo calculation, given a particular set of splitting parameters and transport problem specifications. ONETRAN was also employed to solve for the expected time per 
particle history, thus facilitating a completely deterministic calculation of the Monte Carlo cost function $\left(\sigma_{s}^{2} \tau_{p}\right)$.

Generally speaking, ONETRAN is a program designed to solve the onedimensional, multigroup form of the Boltzmann transport equation in either plane, cylinurical, spherical, or two-angle plane geometries. Both direct and adjoint solutions may be obtained. The code allows for anisotropic sccttering as well as anisotropic inhomogeneous sources (both distributed and boundary sources). Furthermore, the solution may be subject to a variety of possible boundary conditions.

ONETRAN actually solves a set of discrete ordinates equations which are derived from the multigroup transport equation by directly differencing the angular variable. Thus, in standard plane geometry (for example), the continuous angular interval $\mu \varepsilon(-1,1)$ is discretized into a set of quadrature weights and angles. Hence, integrals over $\mu$ are approximated by weighted sums (typically Gauss-Legendre quadrature sets are employed).

The spatial variable is discretized in ONETRAN by employing a linear, discontinuous finite-element scheme. Thus, the solution function (typically the angular particle flux) is considered to be piecewise linear in a given spatial mesh cell and discontinuous across mesh cell boundaries. The actual (true) value of the angular flux on the cell boundary, however, is always taken to be the limit of the flux as the boundary is approached in the direction of particle streaming. A direct solution of a $(2 \times 2)$ system of equations is employed to determine the values of tine two mesh cell-edge fluxes. This system of equations is 
derived by using a suitable weighting and integration technique appliet to the discrete ordinates equations in the space-angle mesh cell. For specific details relating to this method, the reader is directed to Ref. 21 .

The solution strategy in ONETPAN is iterative in nature because the source term of the discrete ordinates equations contains contributions from in-scattering and fission which themselves depend on the angular fluxes being computed. In fact, two nested iterations are used. The outer iteration sweeps through all energy groups and accounts for groupto-group contributions due to scattering and fission. The inner iteration sweeps the space-angle mesh for a single group and accounts for within-group scattering thus coupling all directions.

The heart of the inner iteration, then, is the sweep through the space-angle mesh. The sweep proceeds in the direction of particle flow. At the beginning of the sweep, the boundary condition is used to set the value of the boundary angular flux. Then, for each direction, the spatial mesh is swept either from right to left or left to right, and the mesh cell-edge angular fluxes are computed by solving (in each cell) the $(2 \times 2)$ system of equations referred to in an earlier paragraph. The description of the code given above is of necessity very brief since a more detailed discussion is outside the scope of this dissertation. The reader may consult several good references for a more complete treatment. $20,21,22,23$

ONETRAN is typically employed, then, to compute a deterninistic, global description of the angular particle flux. In this work, however, 
the solution functions are instead the first and second moments of the slab transmission score as well as the expected time per particle history $\left[F_{1}(z, \mu, w), F_{2}(z, \mu, w)\right.$, and $\left.F_{T}(z, \mu, w)\right]$. A one-group calculation in the adjoint mode is considered. Although ONETRAN calculates a globa1 solution, i.e., at all mesh cells and for all discrete directions, for the problem considered here the main quantity of interest is the solution at $z=0$ and $\mu=1$. This is because the Monte Carlo simulation of the transport problem involves a monodirectional (normally incident) source of neutrons at $z=0$. Unfortunate1y, the Gauss quadrature set usua11y used for angular discretization does not include the angular coordinate $\mu=1$. Therefore, a more non-conventional but acceptable Lobatto quadrature $\operatorname{set}^{24}$ was used for the discrete ordinates calculations in this work. This set has the same properties as the Gauss-Legendre set (as far as the $S_{n}$ calculation is concerned); however, it includes a discrete direction corresponding to $\mu=1$.

4.3.3 Required Modifications to ONETRAN. As mentioned previously, the solutions for the second moment of the transmission score and the time per history are associated with a discontinuity at the splitting surface $z=z_{s}$. Effectively, this represents an internal boundary source at $z_{s}$ which is not a standard option in ONETRAN. The appropriate implementation of the discontinuity conditions required a modification in subroutine INNER of ONETRAN (this routine performs the sweep of the space-angle mesh involved in the inner iteration as discussed above).

The solution algorithm incorporated in ONETRAN is explicit in nature. Thus (when considering a forward mode calculation), the mesh is 
swept and unknown; are solved in the direction of particle streaming. The calculation of the mesh cell-edge angular fluxes in a given cell is coupled to the solution in the previous cell by means of an "inflow" quantity from that cell. (The ONETRAN program mnemonic for this quantity is PSIB). Thus, the discontinuity conditions given in Eqs. $(4.1 .2 .34,35)$ are imposed on PSIB at the splitting surface in the course of the spaceangle sweep of the inner iteration.

Specifically, the splitting surface is identified with a particular mesh cell boundary. During the spatial sweep (for a given quadrature angle), a check is made for the particular mesh cell to the immediate left (if $\mu<0$ ) or right (if $\mu>0$ ) of the splitting surface. (It should be noted here, that for adjoint calculations using ONETRAN, the solution of the problem in direction $\mu$ is identified with the adjoint solution in the direction $-\mu^{21}$ ) When this particular cell is reached, the quantity PSIB is modified according to the discontinuity conditions pertaining to the given angle. As an example, fcr $\mu<0$ the discontinuity in the second moment of the transmission score is imposed on PSIB in the mesh cell which has the splitting surface as its right boundary, and

$$
\operatorname{PSIB}=\frac{1}{m} \cdot \operatorname{PSIB}+\mathrm{XJMP},
$$

where XJMP is the quantity inferred from the discontinuity condition stated in Eq. (4.1.2.35). Control is then returned to the regular ONETRAN solution algorithm, which determines the values for the solution function on the right and left boundaries of the mesh cell. The nature of the modification for $\tau_{p}$ calculation is similar; $1 . e .$, the 
discontinuities given in Eqs. $(4.2 .2 .15,16)$ are imposed on PSIB in the appropriate mesh cell which has the splitting surface as a boundary.

\subsection{Numerical $\left(S_{\mathfrak{n}}\right)$ Study for the Case of a Single Splitting Surface}

Results of the numerical study involving one splitting surface are presented in this section. A short description of the parameter survey is followed by an introduction to the special-purpose Monte Carlo code which was written to verify stochastically the predictions of $\sigma_{s}{ }^{2}$ and $\tau_{p}$. The specific event timings which were required for the $\tau_{p}$ calculation are summarized. Finally, the results of the parameter study are presented in graphical and tabular form.

4.4.1 Overview of the Study. Deterministic $S_{n}$ calculations were employed to predict the cost $\left(f_{c}=\sigma_{s}{ }^{2} \tau_{p}\right.$ ) of Monte Carlo calculations for a variety of slab thicknesses ( $\leq 10 \mathrm{mfp}$ ) and scattering ratios. Specifically, scattering fractions of $w_{s}=0.10,0.50$, and 0.90 were studied in conjunction with slab thicknesses of $D=2.00,3.00,5.00$, and 10.0 mean free paths. In each case, it was desired to map out the behavior of the computing cost as a function of the splitting surface location. Various integer values of the splitting ratio (m) were considered. For all cases studied, optimum values for the splitting ratio and splitting surface location were determined.

In order to predict the cost associated with a given set of splitting parameters, the results of three separate $S_{n}$ calculations are required: two for the first two moments of the slab transmission score and one for the time per particle history. Since the first monent of 
the transmission score is not affected by splitting with Russian roulette, this calculation must be made only once for a given slab thickness and particular scattering ratio. It then remains constant over all splitting conditions. Also as discussed previously, the particle weight has no effect on the event scores, i.e., event timings, associated with the $\tau_{p}$ calculation. Thus, since survival biasing is considered, the time per particle history remains constant over all scattering ratios (given a fixed slab thickness and a fixed set of splitting parameters).

4.4.2. Monte Carlo/ $S_{n}$ Comparisons. A special-purpose Monte Carlo code MCS1 (see Appendix F) was written in order to provide stochastic verification of the predicted values for sample variance and time per particle history. The code reflects the features of the transport model as introduced in Chapter III. Thus, a homogeneous semi-infinite slab geometry (D mfp in thickness) is considered. Splitting and Russian roulette take place at a single internal surface. Absorption is treated as an appropriate reduction of particle weight at each collision, and the post-collision direction is randomly sampled from an isotropic-distribution in $\mu$. Source particles are monodirectional [ $\mu$ (source) = 1] and normally incident on the front surface of the slab. The tally is the particle leakage across the back surface of the slab.

Required input to MCS1 (see Appendix F) includes: values for the scattering ratio (WS), total cross section (SIGT), total number of particle histories to be processed (NMAX), locations for the front, splitting, and tally surfaces (SURF array), and values for the importances in the two regions of the geometry (IMP array). The code 1isting 
is presented in Appendix F, and the logical flow chart for the Monte Carlo calculation is included as Fig. $15(\mathrm{a}, \mathrm{b}$, and $\mathrm{c}$ ).

In order to execute the $S_{n}$ calculation for $\tau_{p}$, actual computer timings were required for all the possible events involved in the Monte Carlo calculation. The computer times involved in the processing of a collision $\left(\tau_{c}\right)$, splitting event $\left(\tau_{s}\right)$, Russian roulette event $\left(\tau_{R}\right)$, front-surface escape $\left(\tau_{0}\right)$ and tally-surface escape $\left(\tau_{T}\right)$ were obtained subject to execution or a CDC-7600 computer. The technique involved was to first isolate the section, or block, of Fortran coding (from MCS1) which included all the operations performed in the course of processing the particular event being considered. This block of coding was then repetitively executed inside a D0-10op. System calls which return actual CPU (central processor) time charged to a job were used immediately before and after the DO-loop to determine the total computer time expended inside the DO-loop. Given the number of loops actually made, the time required to execute one pass through the loop was deduced. However, this timing also reflected the time involved in processing extra steps, e.g., the DO-loop logic, which are not really involved in processing the Monte Carlo event being considered. Thus, a separate timing of these extra sceps was made and subsequently subtracted from the original time. Results of the timing studies are displayed in Table 1 and include the times in microseconds as well as "time units" (normalized to $\tau_{c}$ ).

A test case was employed in order to provide a check on the timings. First, the regular version of MCS1 was executed, and time per particle 


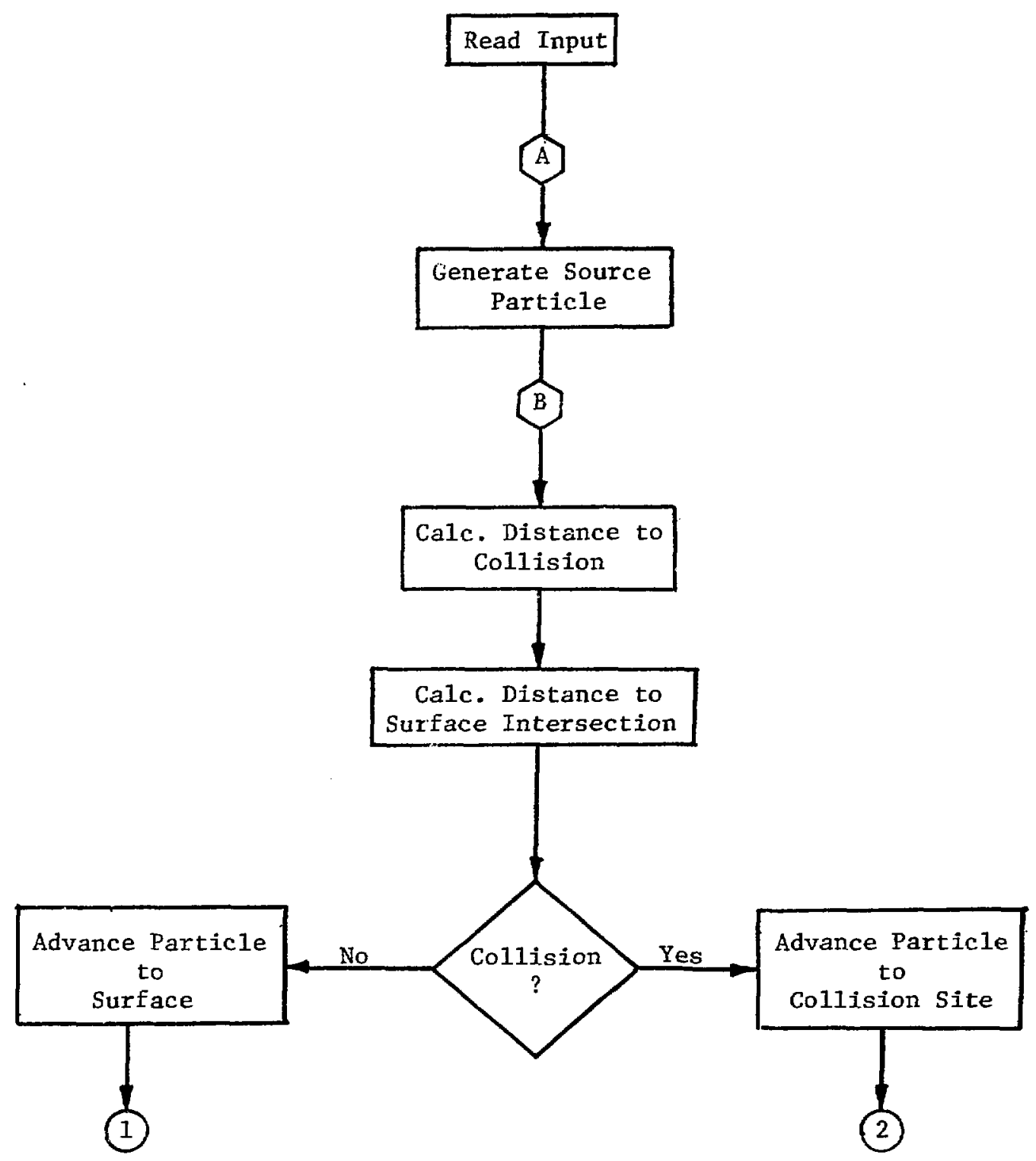

Figure 15a: Logical Flowchart for Specia1-Purpose Monte Carlo Code MCSI. 


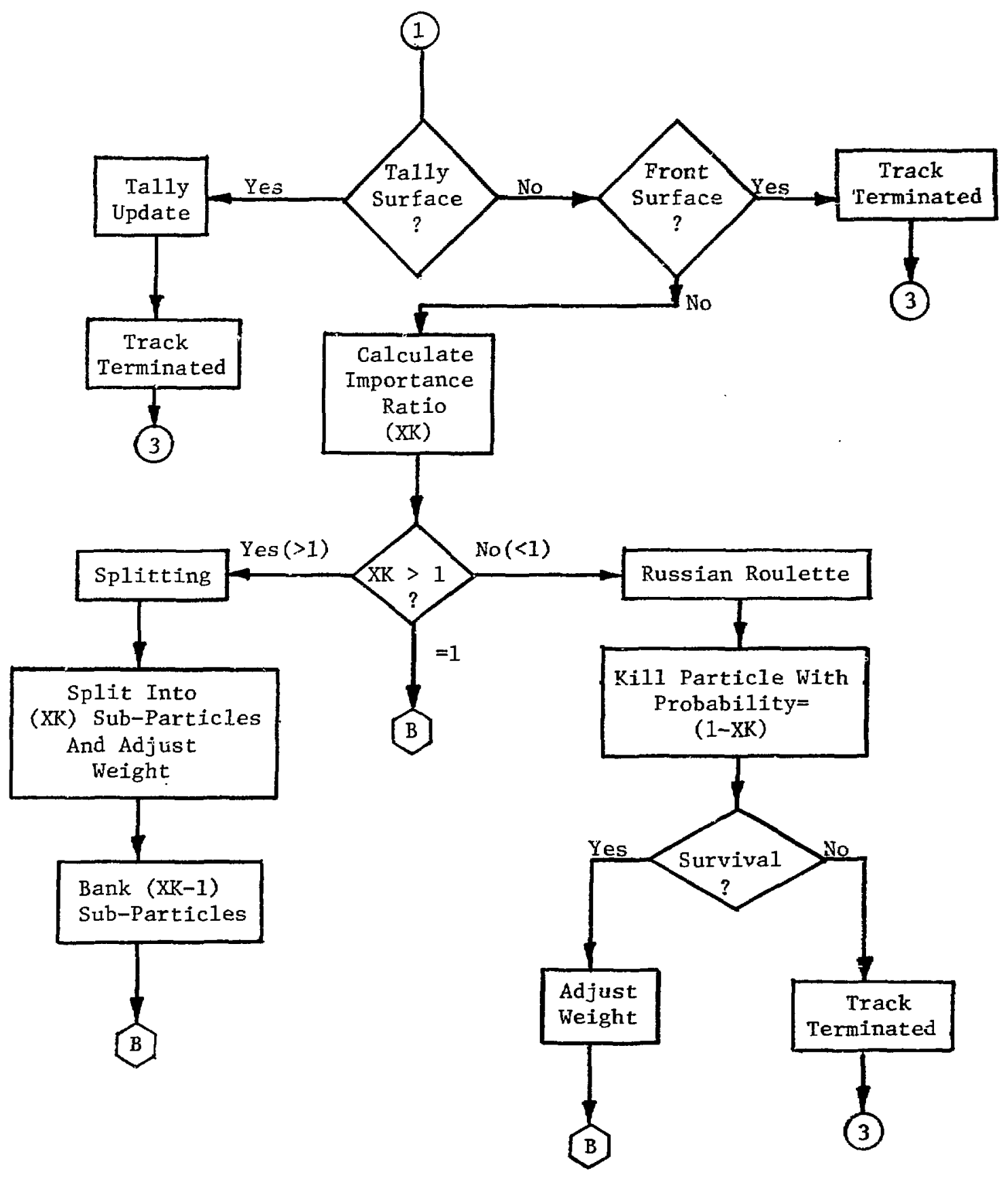

Figure 15b. MCS1 Flowchart, contd. 

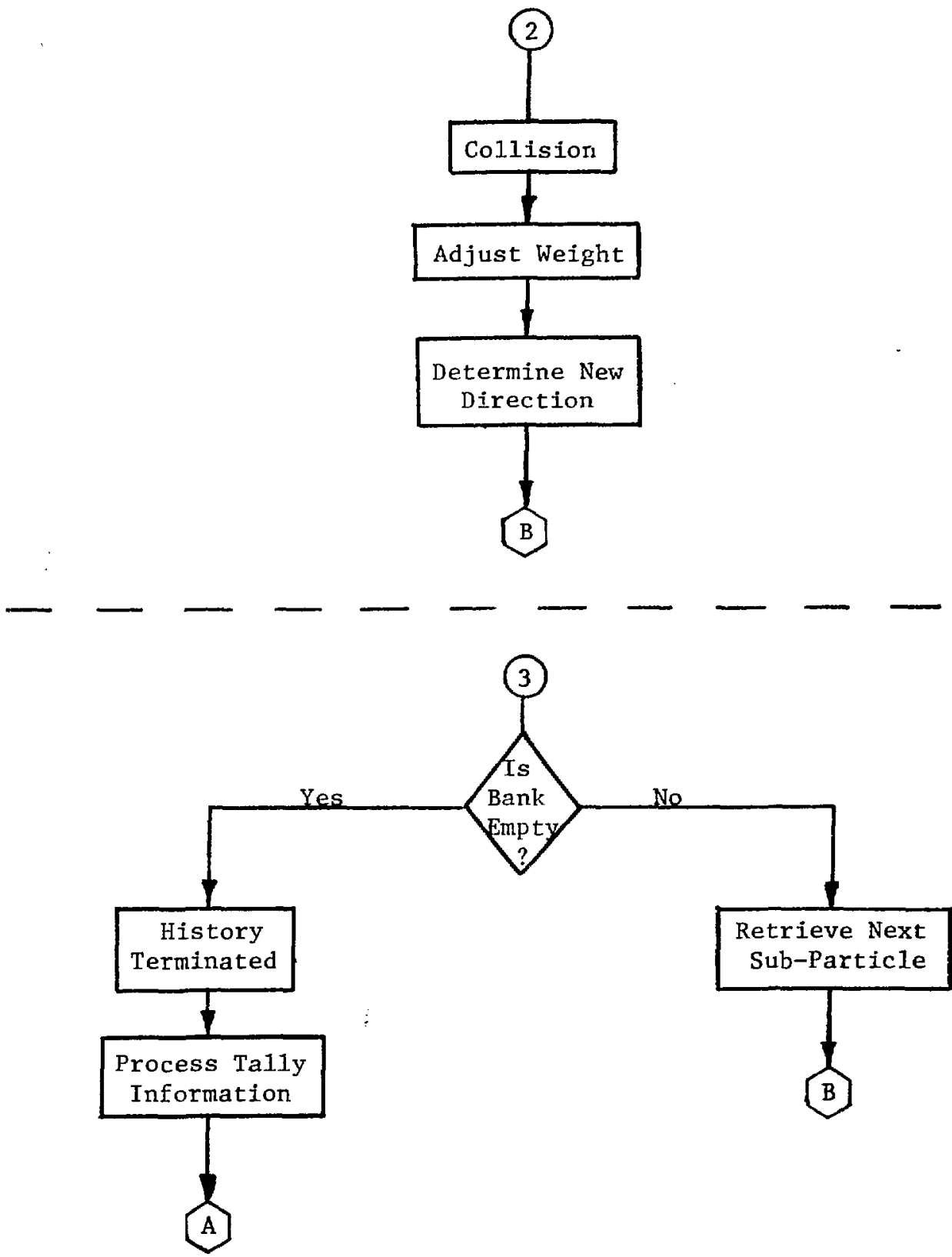

Figure 15c. MCS1 Flowchart, contd. 


\begin{tabular}{|l|c|c|}
\hline Time to Process... & \multicolumn{2}{|c|}{ Time } \\
\cline { 2 - 3 } Collision, $\tau_{c}$ & $11.89 \pm 0.13 \mu \mathrm{sec}$ & Time Units \\
Splitting $(\mathrm{m}=2), \tau_{\mathrm{S}}$ & $22.57 \pm 0.15$ & 1.00 \\
Russian Roulette(m=2), $\tau_{\mathrm{R}}$ & $14.65 \pm 0.08$ & 1.23 \\
Front-Surface Escape, $\tau_{0}$ & $10.99 \pm 0.09$ & 0.92 \\
Tally-Surface Escape, $\tau_{\mathrm{T}}$ & $11.04 \pm 0.11$ & 0.93 \\
Source Particle & $0.59 \pm 0.08$ & 0.05 \\
History Update & $2.24 \pm 0.10$ & 0.19 \\
\hline
\end{tabular}

Table 1. MCS1 Event Timing Summary. 
history was obtained. The same case was then run with a version of MCS1 that had been modified to include event counters. This allowed for the calculation of the expected number of each type of event (per particle history). The expected time per history was then predicted as the product of the expected number of a given event and the time per event (from Table 1) summed over all events. The value calculated in this manner agreed with the actual time per history (obtained from a regular MCS1 calculation) to within $0.7 \%$.

Comparisons between predicted values of the cost function $\left(f_{c}=\sigma_{s}^{2} \tau_{p}\right)$ and stochastic estimates of the quantity are presented in Table 2 (for $D=3.0 \mathrm{mfp}$ ), Table $3(\mathrm{D}=5.0 \mathrm{mfp}$ ), and Table 4 $(D=10.0 \mathrm{mfp})$. In general, the Monte Carlo problems were run long enough to result in an estimate of the slab transmission with a relative error ( $1 \sigma$ confidence level) less than $1 \%$. Unfortunately, this was not possible for the case of the 10.0 mean-free-path slab with scattering ratios of 0.10 and 0.50 . The expenditure of a rea snable amount of computer time only allowed for relative errors that were as low as $15 \%$. As indicated in Tables $2-4$, the agreement between predicted and actual values of $\sigma_{s}{ }^{2}$ and $\tau_{p}$ was quite good. In fact, in most cases the discrepancies were less than $2 \%$ (frequently less than $1 \%$ ). As mentioned above, for the cases of $10.0 \mathrm{mfp}$ with moderate to heavy absorption $\left(w_{S}=0.10\right.$ and 0.50$)$, practical limitations on computer time restricted the accuracy of the Monte Carlo calculations. It was found from other cases that truly good agreement in the sample varlances was obtained when the Monte Carlo estimate of the transmission was calculated to a 


\begin{tabular}{|c|c|c|c|c|}
\hline CASE & & $\begin{array}{l}\text { Monte Carlo } \\
\text { (MCS1) }\end{array}$ & $\begin{array}{c}s_{n} \\
\text { (ONETRAN) } \\
\end{array}$ & $\%$ Diff. \\
\hline $\begin{array}{l}w_{s}=0.10 \\
z_{s}=0.50 \mathrm{mfp} \\
m=2\end{array}$ & $\begin{array}{l}\sigma_{s}^{2} \\
\tau_{p} \\
f_{c}\end{array}$ & $\begin{array}{l}2.460 \mathrm{E}-02 \\
1.850 \mathrm{E}-04(\mathrm{sec}) \\
4.551 \mathrm{E}-06(\mathrm{sec})\end{array}$ & $\begin{array}{l}2.452 \mathrm{E}-02 \\
1.886 \mathrm{E}-04(\mathrm{sec}) \\
4.624 \mathrm{E}-06(\mathrm{sec})\end{array}$ & $\begin{array}{r}-0.33 \\
+1.91 \\
+1.58\end{array}$ \\
\hline $\begin{array}{l}w_{s}=0.10 \\
z_{s}=1.75 \text { mf } \\
m=2\end{array}$ & $\begin{array}{l}\sigma_{s}^{2} \\
\tau_{p} \\
f_{c}\end{array}$ & $\begin{array}{l}3.002 \mathrm{E}-02 \\
1.359 \mathrm{E}-04 \\
4.080 \mathrm{E}-06\end{array}$ & $\begin{array}{l}2.391 \mathrm{E}-02 \\
1.381 \mathrm{E}-04 \\
4.130 \mathrm{E}-06\end{array}$ & $\begin{array}{l}-0.37 \\
+1.59 \\
+1.21\end{array}$ \\
\hline $\begin{array}{l}w_{s}=0.10 \\
z_{s}=2.50 \mathrm{mf} p \\
m=2\end{array}$ & $\begin{array}{l}\sigma_{s}^{2} \\
\tau_{p} \\
f_{c}\end{array}$ & $\begin{array}{l}3.807 \mathrm{E}-02 \\
1.117 \mathrm{E}-04 \\
4.252 \mathrm{E}-06\end{array}$ & $\begin{array}{l}3.796 \mathrm{E}-02 \\
1.129 \mathrm{E}-04 \\
4.286 \mathrm{E}-06\end{array}$ & $\begin{array}{r}-0.29 \\
+1.06 \\
+0.79\end{array}$ \\
\hline $\begin{array}{l}w_{s}=0.10 \\
z_{s}=1.25 \mathrm{mfp} \\
m=3\end{array}$ & $\begin{array}{l}\sigma_{\mathrm{s}}^{2} \\
\mathrm{\tau}_{\mathrm{p}} \\
\mathrm{f}_{\mathrm{c}}\end{array}$ & $\begin{array}{l}2.018 \mathrm{E}-02 \\
2.018 \mathrm{E}-04 \\
4.072 \mathrm{E}-06\end{array}$ & $\begin{array}{l}2.020 E-02 \\
2.064 E-04 \\
4.169 E-06\end{array}$ & $\begin{array}{l}+0.10 \\
+2.22 \\
+2.33\end{array}$ \\
\hline $\begin{array}{l}w_{s}=0.10 \\
z_{s}=1.50 \mathrm{mfp} \\
m=4\end{array}$ & $\begin{array}{l}\sigma_{s}^{2} \\
\tau_{p} \\
f_{c}\end{array}$ & $\begin{array}{l}1.884 \mathrm{E}-02 \\
2.241 \mathrm{E}-04 \\
4.222 \mathrm{E}-06\end{array}$ & $\begin{array}{l}\text { I. } 876 \mathrm{E}-02 \\
2.288 \mathrm{E}-04 \\
4.292 \mathrm{E}-06\end{array}$ & $\begin{array}{l}-0.43 \\
+2.05 \\
+1.63\end{array}$ \\
\hline $\begin{array}{l}w_{s}=0.50 \\
z_{s}=1.75 \text { mf } \\
m=2\end{array}$ & $\begin{array}{l}\sigma_{s}^{2} \\
\tau_{p} \\
f_{c}\end{array}$ & $\begin{array}{l}3.603 \mathrm{E}-02 \\
1.359 \mathrm{E}-04 \\
4.896 \mathrm{E}-06\end{array}$ & $\begin{array}{l}3.594 \mathrm{E}-02 \\
1.381 \mathrm{E}-04 \\
4.963 \mathrm{E}-06\end{array}$ & $\begin{array}{l}-0.25 \\
+1.59 \\
+1.35\end{array}$ \\
\hline $\begin{array}{l}w_{s}=0.50 \\
z_{s}=0.50 \mathrm{mfp} \\
m=4\end{array}$ & $\begin{array}{l}\sigma_{s}^{2} \\
\tau_{p} \\
f_{c}\end{array}$ & $\begin{array}{l}1.571 \mathrm{E}-02 \\
3.315 \mathrm{E}-04 \\
5.208 \mathrm{E}-06\end{array}$ & $\begin{array}{l}1.568 \mathrm{E}-02 \\
3.384 \mathrm{E}-04 \\
5.306 \mathrm{E}-06\end{array}$ & $\begin{array}{l}-0.19 \\
+2.04 \\
+1.85\end{array}$ \\
\hline $\begin{array}{l}w_{s}=0.90 \\
z_{s}=1.75 \mathrm{mfp} \\
m=2\end{array}$ & $\begin{array}{l}\sigma_{s}^{2} \\
\tau_{p} \\
f_{c}\end{array}$ & $\begin{array}{l}8.473 \mathrm{E}-02 \\
1.365 \mathrm{E}-04 \\
1.156 \mathrm{E}-05\end{array}$ & $\begin{array}{l}8.482 E-02 \\
1.381 E-04 \\
1.171 E-05\end{array}$ & $\begin{array}{l}+0.11 \\
+1.16 \\
+1.28\end{array}$ \\
\hline $\begin{array}{l}w_{s}=0.90 \\
z_{s}=0.25 \mathrm{mfp} \\
\mathrm{m}=4\end{array}$ & $\begin{array}{l}\sigma_{\mathrm{s}}^{2} \\
{ }_{\mathrm{p}}^{\mathrm{p}} \\
\mathrm{f}_{\mathrm{c}}\end{array}$ & $\begin{array}{l}3.278 \mathrm{E}-02 \\
3.588 \mathrm{E}-04 \\
1.176 \mathrm{E}-05\end{array}$ & $\begin{array}{l}3.288 \mathrm{E}-02 \\
3.646 \mathrm{E}-04 \\
1.199 \mathrm{E}-05\end{array}$ & $\begin{array}{l}+0.29 \\
+1.59 \\
+1.91\end{array}$ \\
\hline
\end{tabular}

Table 2. Monte Carlo/s Comparisons ( $D=3.0 \mathrm{mfp}$ )

$$
\left[f_{c}=\sigma_{s}^{2} \tau_{p}(s e c)\right]
$$




\begin{tabular}{|c|c|c|c|c|}
\hline CASE & & $\begin{array}{l}\text { Monte Carlo } \\
\text { (MCS1) }\end{array}$ & $\begin{array}{c}s_{n} \\
\text { (ONETRAN) }\end{array}$ & \% Diff. \\
\hline $\begin{array}{l}w_{s}=0.10 \\
z_{s}=0.50 \mathrm{mfp} \\
m=2\end{array}$ & $\begin{array}{l}\sigma_{s}^{2} \\
\tau_{p} \\
f_{c}\end{array}$ & 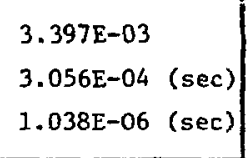 & 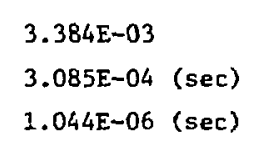 & $\begin{array}{l}-0.38 \\
+0.94 \\
+0.57\end{array}$ \\
\hline $\begin{array}{l}w_{s}=0.10 \\
z_{s}=4.00 \mathrm{mfp} \\
m=2\end{array}$ & $\begin{array}{l}\sigma_{s}^{2} \\
\tau_{p} \\
f_{c}\end{array}$ & $\begin{array}{l}4.664 \mathrm{E}-03 \\
1.767 \mathrm{E}-04 \\
8.241 \mathrm{E}-07\end{array}$ & $\begin{array}{l}4.654 E-03 \\
1.755 E-04 \\
8.185 E-07\end{array}$ & $\begin{array}{l}20 \\
-0.68 \\
-0.68\end{array}$ \\
\hline $\begin{array}{l}w_{s}=0.10 \\
z_{s}=2.50 \mathrm{mfp} \\
\pi=6\end{array}$ & $\begin{array}{l}\sigma_{s}^{2} \\
\tau_{p} \\
f_{c}\end{array}$ & $\begin{array}{l}1.512 \mathrm{E}-03 \\
4.511 \mathrm{E}-04 \\
7.271 \mathrm{E}-07\end{array}$ & $\begin{array}{l}1.594 \mathrm{E}-03 \\
4.538 \mathrm{E}-04 \\
7.233 \mathrm{E}-07\end{array}$ & $\begin{array}{l}-1.13 \\
+0.59 \\
-0.52\end{array}$ \\
\hline $\begin{array}{l}w_{s}=0.50 \\
z_{s}=3.00 \mathrm{mfp} \\
m=3\end{array}$ & $\begin{array}{l}\sigma_{s}^{2} \\
\tau_{p} \\
f_{c}\end{array}$ & $\begin{array}{l}4.029 \mathrm{E}-03 \\
2.455 \mathrm{E}-04 \\
9.891 \mathrm{E}-07\end{array}$ & $\begin{array}{l}4.012 \mathrm{E}-03 \\
2.480 \mathrm{E}-04 \\
9.949 \mathrm{E}-07\end{array}$ & $\begin{array}{l}-0.42 \\
+1.00 \\
+0.58\end{array}$ \\
\hline $\begin{array}{l}w_{s}=0.50 \\
z_{s}=1.00 \mathrm{mfp} \\
m=3\end{array}$ & $\begin{array}{l}\sigma_{s}^{2} \\
\tau_{p} \\
f_{c}\end{array}$ & $\begin{array}{l}2.934 \mathrm{E}-03 \\
3.964 \mathrm{E}-04 \\
1.163 \mathrm{E}-06\end{array}$ & $\begin{array}{l}2.949 E-03 \\
4.005 E-04 \\
1.181 E-06\end{array}$ & $\begin{array}{l}+0.51 \\
+1.02 \\
+1.52\end{array}$ \\
\hline $\begin{array}{l}w_{s}=0.50 \\
z_{s}=3.00 \mathrm{mfp} \\
m=4\end{array}$ & $\begin{array}{l}\sigma_{s}^{2} \\
\tau_{p} \\
E_{c}\end{array}$ & $\begin{array}{l}3.462 E-03 \\
2.886 E-04 \\
9.991 E-07\end{array}$ & $\begin{array}{l}3.464 \mathrm{E}-03 \\
2.904 \mathrm{E}-04 \\
1.006 \mathrm{E}-06\end{array}$ & $\begin{array}{l}+0.06 \\
+0.62 \\
+0.69\end{array}$ \\
\hline $\begin{array}{l}w_{s}=0.90 \\
z_{s}=1.00 \mathrm{mfp} \\
m=2\end{array}$ & $\begin{array}{l}\sigma_{s}^{2} \\
\tau_{p} \\
f_{c}\end{array}$ & $\begin{array}{l}1.925 \mathrm{E}-02 \\
2.850 \mathrm{E}-04 \\
5.486 \mathrm{E}-06\end{array}$ & $\begin{array}{l}1.934 E-02 \\
2.873 E-04 \\
5.556 E-06\end{array}$ & $\begin{array}{l}+0.46 \\
+0.80 \\
+1.26\end{array}$ \\
\hline $\begin{array}{l}w_{s}=0.90 \\
z_{s}=4.00 \\
m=2\end{array}$ & $\begin{array}{l}\sigma_{s}^{2} \\
\tau_{p} \\
f_{c}\end{array}$ & $\begin{array}{l}2.996 E-02 \\
1.768 E-04 \\
5.297 E-06\end{array}$ & $\begin{array}{l}3.011 \mathrm{E}-02 \\
1.755 \mathrm{E}-04 \\
5.284 \mathrm{E}-06\end{array}$ & $\begin{array}{l}+0.50 \\
-0.74 \\
-0.25\end{array}$ \\
\hline $\begin{array}{l}w_{s}=0.90 \\
z_{s}=2.00 \\
m=4\end{array}$ & $\begin{array}{l}\sigma_{s}^{2} \\
\tau_{p} \\
f_{c}\end{array}$ & $\begin{array}{l}1.600 \mathrm{E}-02 \\
3.891 \mathrm{E}-04 \\
6.226 \mathrm{E}-06\end{array}$ & $\begin{array}{l}1.601 E-02 \\
3.928 E-04 \\
6.289 E-06\end{array}$ & $\begin{array}{l}+0.06 \\
+0.94 \\
+1.00\end{array}$ \\
\hline
\end{tabular}

Tab1e 3. Monte Car1o/s Comparisons ( $D^{=} 5.0 \mathrm{mfp}$ )

$$
\left[f_{c}=\sigma_{s}^{2} \tau_{p}(s e c)\right]
$$




\begin{tabular}{|c|c|c|c|c|}
\hline CASE & & $\begin{array}{l}\text { Monte Carlo } \\
\text { (MCS1) }\end{array}$ & $s_{n}$ & \% Diff. \\
\hline $\begin{array}{l}w_{s}=0.10 \\
z_{s}=2.00 \mathrm{mfp} \\
m=2\end{array}$ & $\begin{array}{l}\sigma_{s}^{2} \\
\tau_{p} \\
f_{c}\end{array}$ & $\begin{array}{l}2.018 \mathrm{E}-05 \\
5.414 \mathrm{E}-04(\mathrm{sec}) \\
1.093 \mathrm{E}-08(\mathrm{sec})\end{array}$ & $\begin{array}{l}2.294 \mathrm{E}-05 \\
5.383 \mathrm{E}-04(\mathrm{sec}) \\
1.235 \mathrm{E}-08(\mathrm{sec})\end{array}$ & $\begin{array}{l}+12.0 \\
-0.57 \\
+11.5\end{array}$ \\
\hline $\begin{array}{l}w_{s}=0.10 \\
z_{s}=3.00 \mathrm{mfp} \\
m=4\end{array}$ & $\begin{array}{l}\sigma_{s}^{2} \\
\tau_{p} \\
f_{c}\end{array}$ & $\begin{array}{l}1.073 \mathrm{E}-05 \\
8.249 \mathrm{E}-04 \\
8.851 \mathrm{E}-09\end{array}$ & $\begin{array}{l}1.150 E-05 \\
8.307 E-04 \\
9.556 E-09\end{array}$ & $\begin{array}{l}+6.69 \\
+0.70 \\
+7.38\end{array}$ \\
\hline $\begin{array}{l}w_{s}=0.10 \\
z_{s}=7.00 \text { mfp } \\
m=4\end{array}$ & $\begin{array}{l}\sigma_{s}^{2} \\
\tau_{p} \\
f_{c}\end{array}$ & $\begin{array}{l}1.450 \mathrm{E}-05 \\
4.375 \mathrm{E}-04 \\
6.344 \mathrm{E}-09\end{array}$ & $\begin{array}{l}1.339 \mathrm{E}-05 \\
4.363 \mathrm{E}-04 \\
5.844 \mathrm{E}-99\end{array}$ & $\begin{array}{l}-8.30 \\
-0.28 \\
-8.55\end{array}$ \\
\hline $\begin{array}{l}w_{s}=0.10 \\
z_{s}=7.00 \mathrm{mfp} \\
m=6\end{array}$ & $\begin{array}{l}\sigma_{s}^{2} \\
\tau_{p} \\
f_{c}\end{array}$ & $\begin{array}{l}1.046 \mathrm{E}-05 \\
5.275 \mathrm{E}-04 \\
5.518 \mathrm{E}-09\end{array}$ & $\begin{array}{l}9.785 E-06 \\
5.221 E-04 \\
5.109 E-09\end{array}$ & $\begin{array}{l}-6.90 \\
-1.03 \\
-8.00\end{array}$ \\
\hline $\begin{array}{l}w_{s}=0.50 \\
z_{s}=7.00 \mathrm{mfp} \\
m=2\end{array}$ & $\begin{array}{l}\sigma_{s}^{2} \\
\tau_{p} \\
f_{c}\end{array}$ & $\begin{array}{l}3.396 \mathrm{E}-05 \\
3.578 \mathrm{E}-04 \\
1.215 \mathrm{E}-08\end{array}$ & $\begin{array}{l}3.542 \mathrm{E}-05 \\
3.505 \mathrm{E}-04 \\
1.241 \mathrm{E}-08\end{array}$ & $\begin{array}{l}+4.12 \\
-2.08 \\
+2.09\end{array}$ \\
\hline $\begin{array}{l}w_{s}=0.50 \\
z_{s}=5.00 \mathrm{mfp} \\
m=4\end{array}$ & $\begin{array}{l}\sigma_{s}^{2} \\
\tau_{p} \\
f_{c}\end{array}$ & $\begin{array}{l}1.656 \mathrm{E}-05 \\
6.049 \mathrm{E}-04 \\
1.002 \mathrm{E}-08\end{array}$ & $\begin{array}{l}1.699 \mathrm{E}-05 \\
6.032 \mathrm{E}-04 \\
1.025 \mathrm{E}-08\end{array}$ & $\begin{array}{l}+2.53 \\
-0.28 \\
+2.24\end{array}$ \\
\hline $\begin{array}{l}w_{s}=0.90 \\
z_{s}=2.00 \mathrm{mfp} \\
m=3\end{array}$ & $\begin{array}{l}\sigma_{s}^{2} \\
f_{p}\end{array}$ & $\begin{array}{l}4.592 \mathrm{E}-04 \\
7.489 \mathrm{E}-04 \\
3.439 \mathrm{E}-07\end{array}$ & $\begin{array}{l}4.556 \mathrm{E}-04 \\
7.513 \mathrm{E}-04 \\
3.423 \mathrm{E}-07\end{array}$ & $\begin{array}{l}-0.79 \\
+0.32 \\
-0.47\end{array}$ \\
\hline $\begin{array}{l}w_{s}=0.90 \\
z_{s}=4.00 \mathrm{mfp} \\
m=3\end{array}$ & $\begin{array}{l}\sigma_{s}^{2} \\
\tau_{p} \\
f_{c}\end{array}$ & $\begin{array}{l}5.200 \mathrm{E}-04 \\
5.814 \mathrm{E}-04 \\
3.023 \mathrm{E}-07\end{array}$ & $\begin{array}{l}5.191 E-04 \\
5.793 E-04 \\
3.007 E-07\end{array}$ & $\begin{array}{l}-0.17 \\
-0.36 \\
-0.53\end{array}$ \\
\hline $\begin{array}{l}w_{s}=0.90 \\
z_{s}=8.00 \mathrm{mfp} \\
m=3\end{array}$ & $\begin{array}{l}\sigma_{s}^{2} \\
\tau_{p} \\
f_{c}\end{array}$ & $\begin{array}{l}8.951 \mathrm{E}-04 \\
3.560 \mathrm{E}-04 \\
3.186 \mathrm{E}-07\end{array}$ & $\begin{array}{l}8.927 E-04 \\
3.521 E-04 \\
3.143 E-07\end{array}$ & $\begin{array}{l}-0.27 \\
-1.11 \\
-1.38\end{array}$ \\
\hline
\end{tabular}

Table 4. Monte Carlo/s Comparisons ( $D=10.0 \mathrm{mfp})$

$$
\left[\mathrm{f}_{\mathrm{c}}=\sigma_{\mathrm{s}}^{2} \tau_{\mathrm{p}}(\mathrm{sec})\right]
$$


relative error of $1 \%$ or better. This was impractical to do for some of the very deep-penetration problems. As an example, some of the cases involving the $10 \mathrm{mfp}$ slab with $\mathrm{w}_{\mathrm{s}}=0.10$ would have taken more than 13 hours of CDC-7600 computer time to process to a relative error of $1 \%$. Thus, the larger discrepancies between predicted and Monte Carlo values of sample variance in such cases (see Table 4) reflected the variance of the variance in the Monte Carlo estimates themselves.

For the smallest slab thicknesses, the time per history was consistent1y overpredicted by the $s_{n}$ calculations by as much as a few percent. Most probably, this was a reflection of an inaccuracy in one (or some) of the event timings which may become more noticeable when the overall number of events in a particle history becomes small (thin slab problems). However, the magnitude of the discrepancy was always quite small (never, for example, affecting the shapes of the cost function curves). In addition, values of $\tau_{p}$ obtained from the Monte Carlo calculations were always subject to normal variations in processing time brought about by changes in the job load in the time-sharing computer environment. Thus, values of $\tau_{p}$ for the same Monte Carlo calculation varied by as much as 1\% depending on the time of day the problem was run. For these reasons, an exact refinement of the computer timings was considered to be outside the scope of this dissertation. Figure 16 illustrates the behavior of the computing cost as a function of splitting surface location for $D=5.0 \mathrm{mf}_{\mathrm{p}}, \mathrm{w}_{\mathrm{s}}=0.50$, and $\mathrm{m}=2$. The solid curve ts a fit to the $\mathrm{s}_{\mathbf{n}^{-}}$ calculated values of $\sigma_{s}{ }^{2} \tau_{p}$. Also plotted in Fig. 16 are stochastic values of $\sigma_{s}{ }^{2} \tau_{p}$ resulting from the Monte Carlo calculations. This figure 


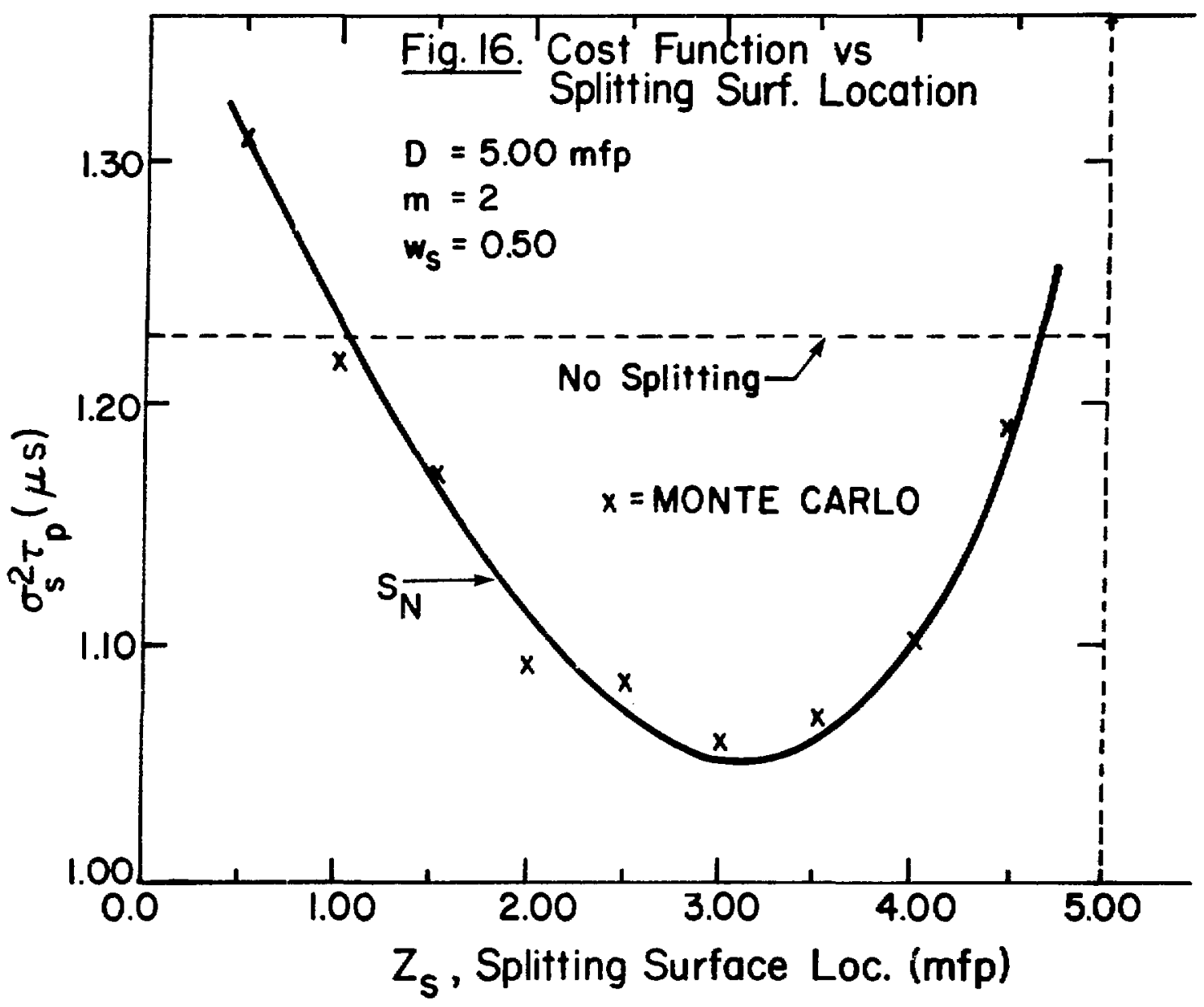


further emphasizes the good agreement between deterministic predictions and actual values of computing cost.

\subsubsection{Single-Surface Results. Results are presented here 11lus-} trating the behavior of the cost function $\left(\sigma_{s}{ }^{2} \tau_{p}\right)$ under a large varfety of transport problem specifications and splitting conditions. The discrete ordinates transport code ONETRAN was employed in generating this information. $\mathrm{P}_{0}-\mathrm{S}_{8}$ calculations (employing a Lobatto quadrature set) with a fine-mesh interval spacing of $\Delta z=0.05$ ifp gave very satisfactory results. The convergence criterion for the first and second moments of the transmission score was set at $1.0 \mathrm{E}-05$. Since the $\tau_{p}$ problem took considerably longer to run, the convergence criterion for that calculation was relaxed slightly to $5.0 \mathrm{E}-05$.

The curves presented in Figs. 17 through 28 represent analytical (polynomial) fits to the $S_{n}$-calculated values of $\sigma_{s}^{2} \tau_{p}$. The abscissa in these curves is the normalized splitting surface location, i.e., $z_{s}$ /D. A Fortran code (COSTFT; see Appendix G) was employed to perform a multiple linear regression (least squares) analysis in fitting the $\mathrm{S}_{\mathrm{n}}$-generated values. A discussion of the regression technique and the appropriate subroutines used in COSTFT appear in Reference 25 (pp. 171176). In all cases, a third- or fourth-order polynomial was sufficient to provide an excellent fit, i.e., to result in a multiple linearcorrelation coefficient of 0.9997 or better.

Figure 23 illustrates the typical case for which splitting in Monte Carlo is cost-effective relative to the non-splitting calculation. 


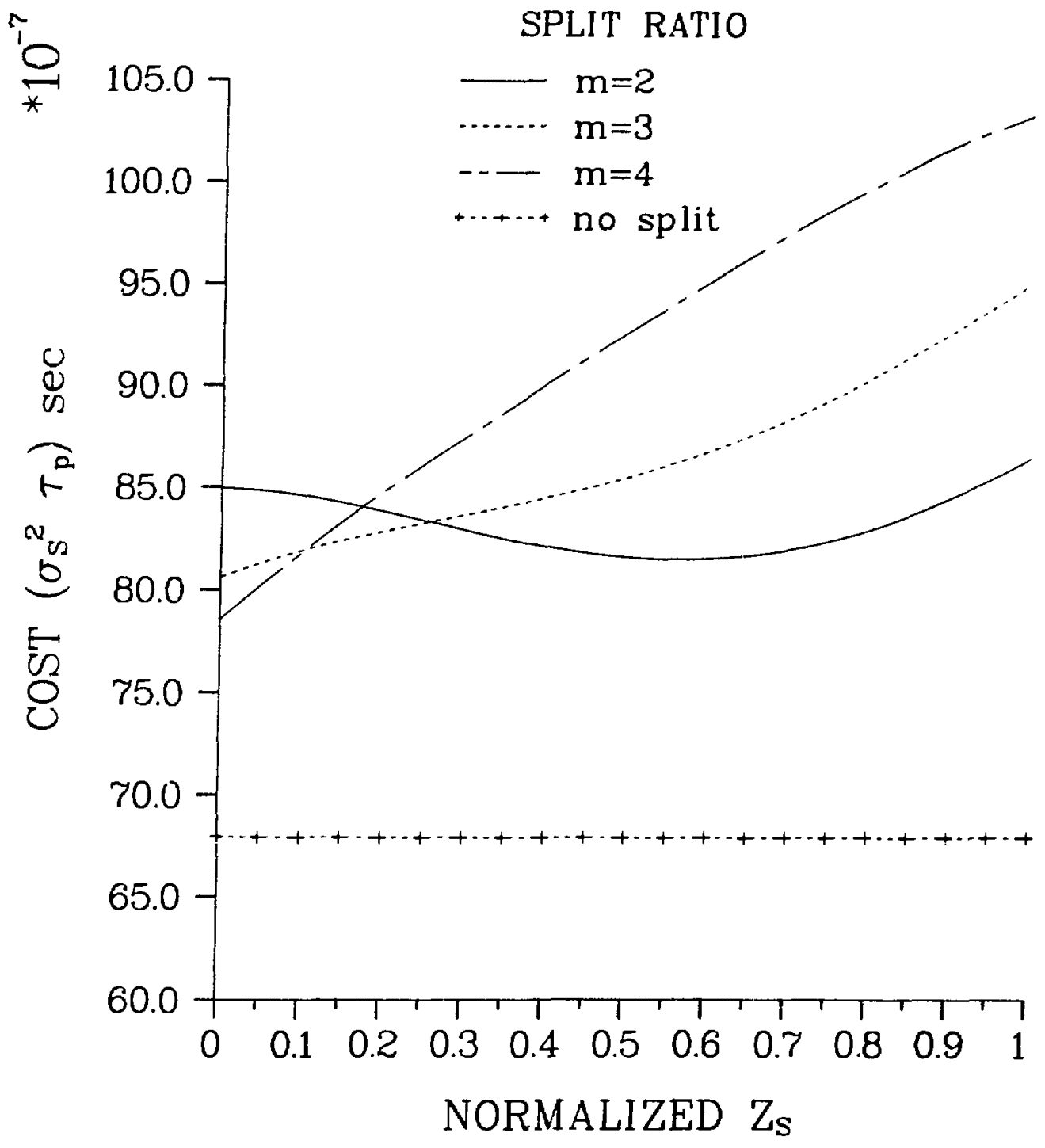

COST vs SPLITTING SURFACE LOCATION $\left(D=2.00, W_{s}=0.10\right)$

Figure 17 


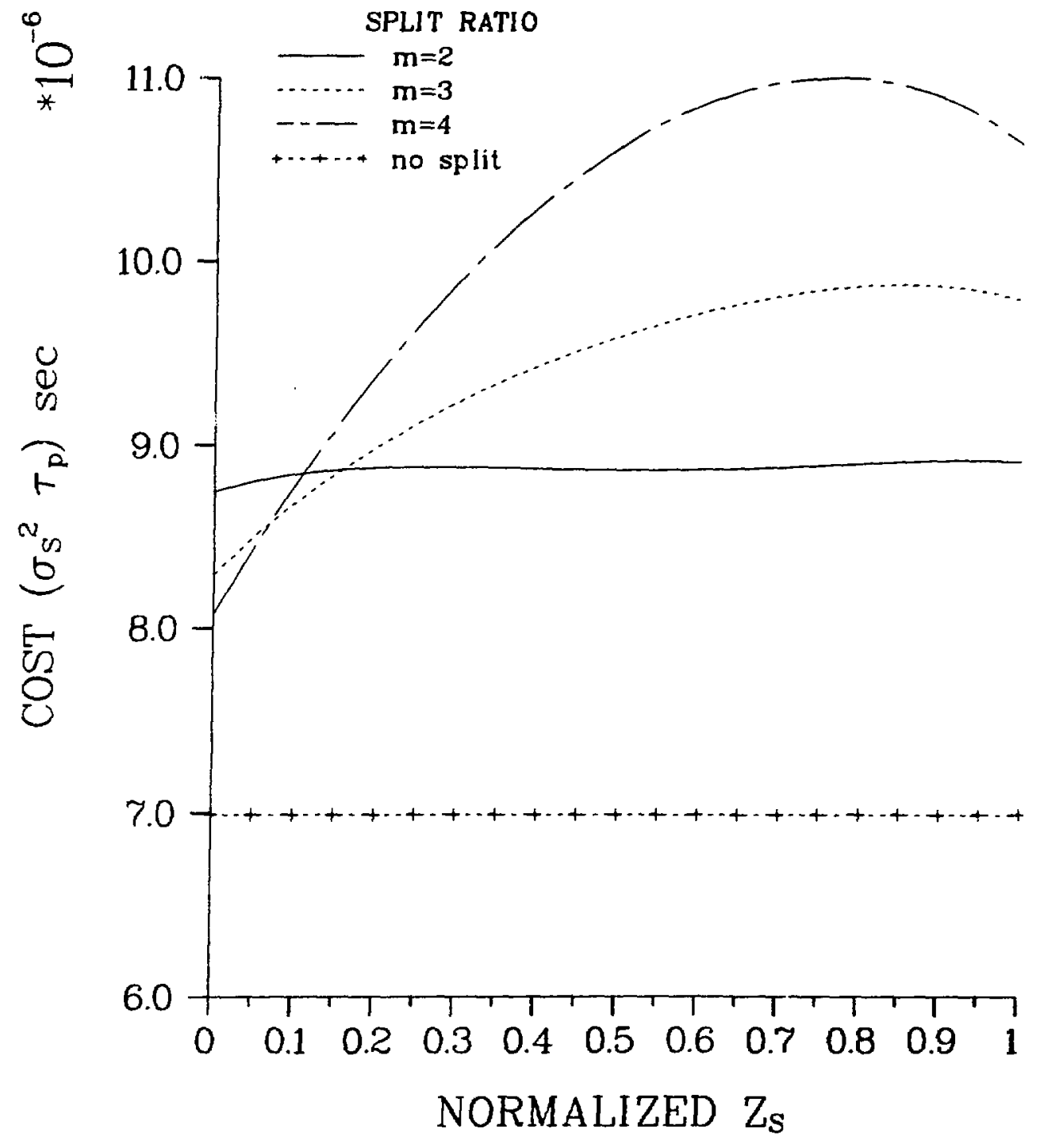

COST vs SPLITTING SURFACE LOCATION $(D=2.00, W s=0.50)$

Figure 18 


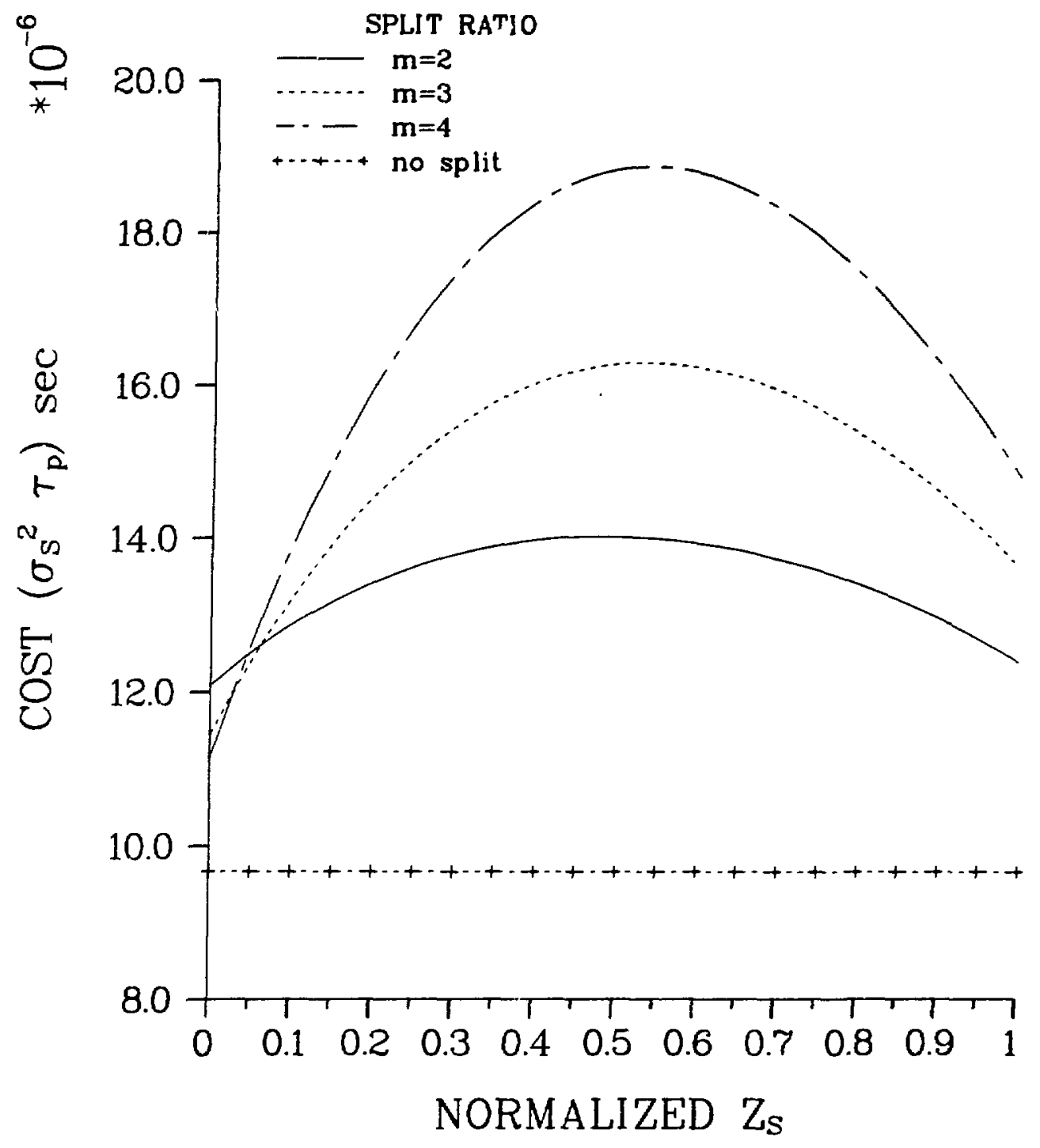

COST vs SPLITTING SURFACE LOCATION
$(D=2.00$, Ws $=0.90)$

Figure 19 


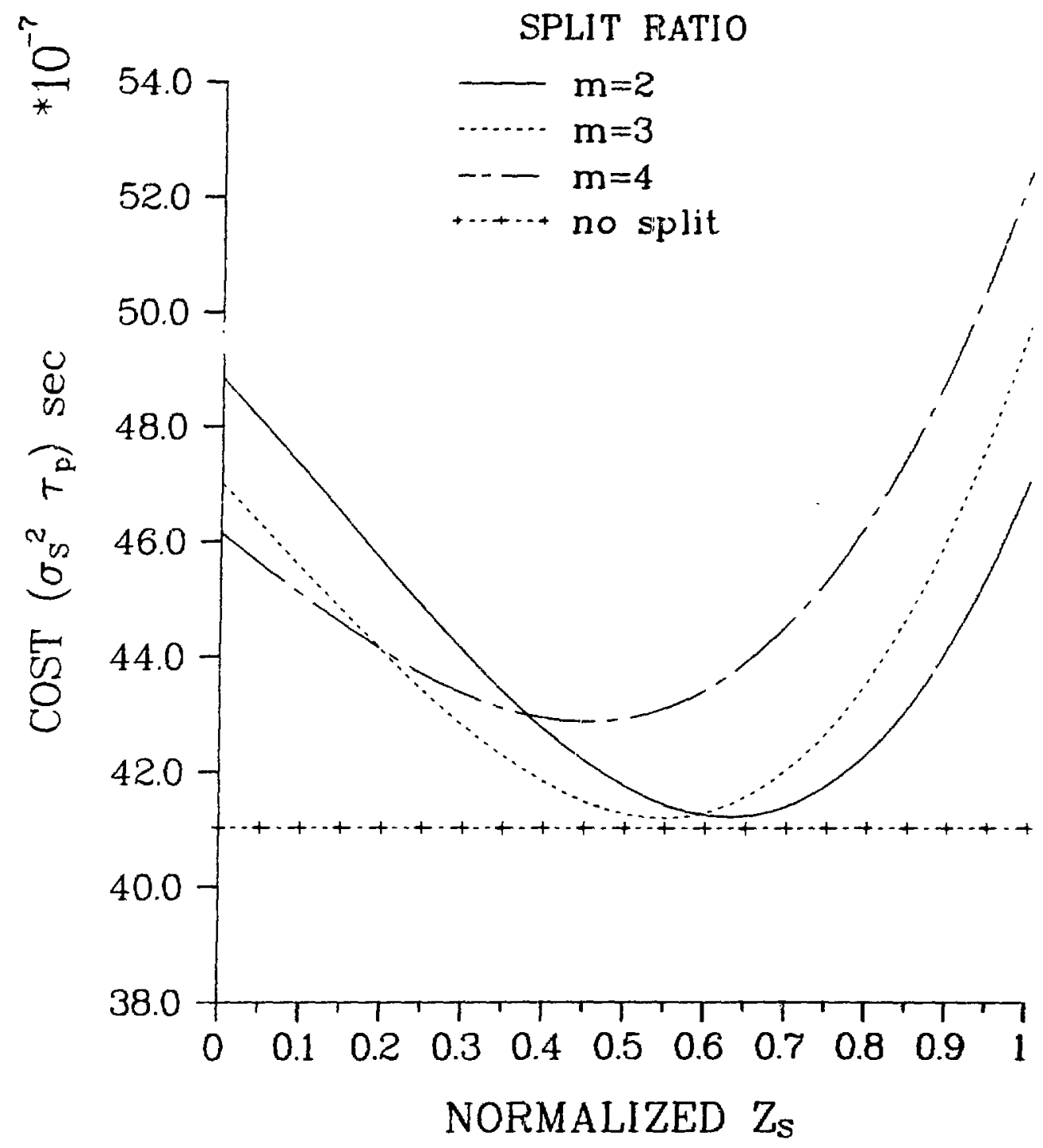

COST vS SPLITTING SURFACE LOCATION $\left(D=3.00, W_{S}=0.10\right)$

Figure 20 


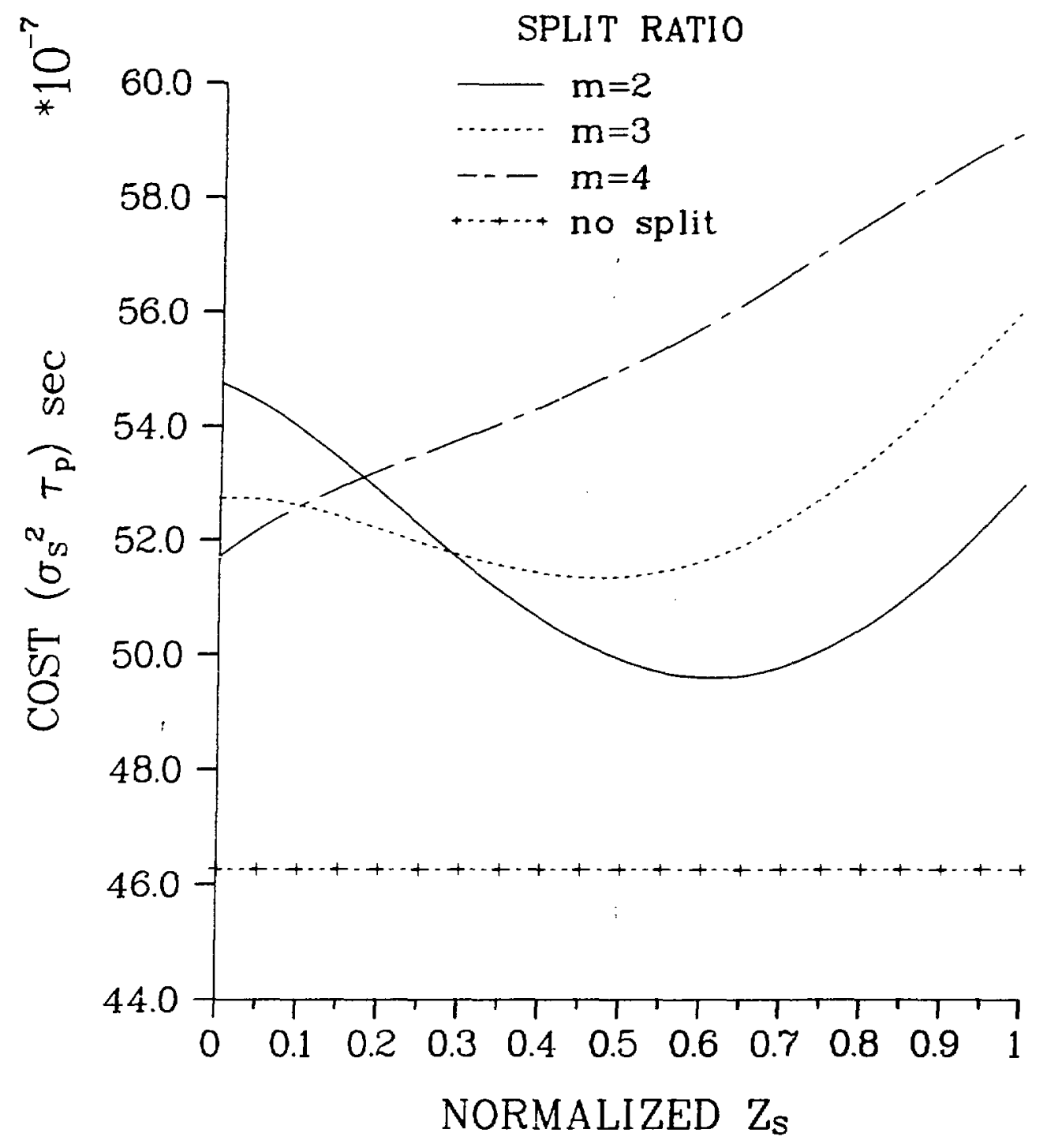

COST vS SPLITTING SURFACE LOCATION $\left(\mathrm{D}=3.00, \mathrm{Ws}_{\mathrm{s}}=0.50\right)$

Figure 21 


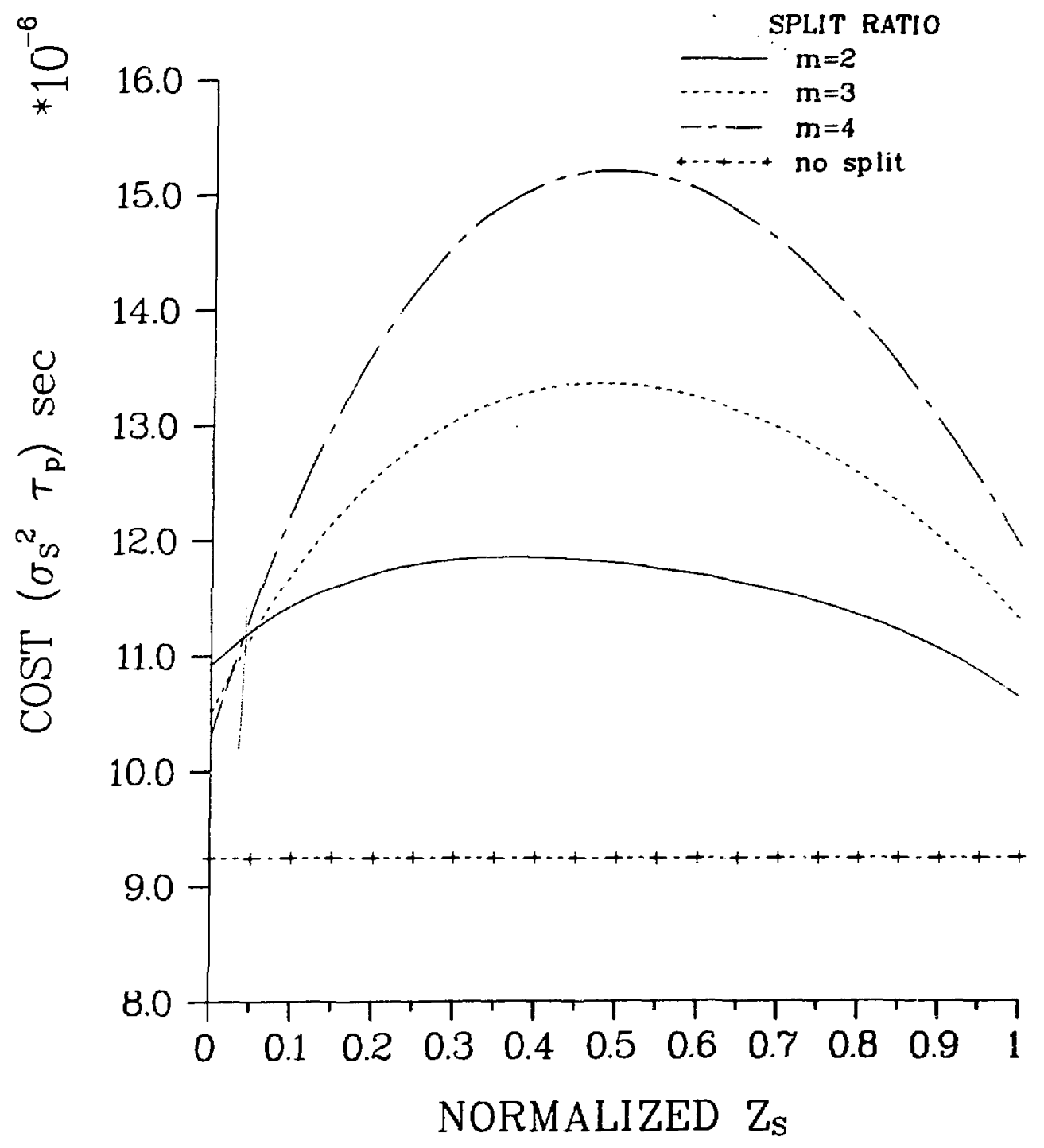

COST VS SPLITTING SURFACE LOCATION $\left(D=3.00, W_{s}=0.90\right)$

Figure 22 


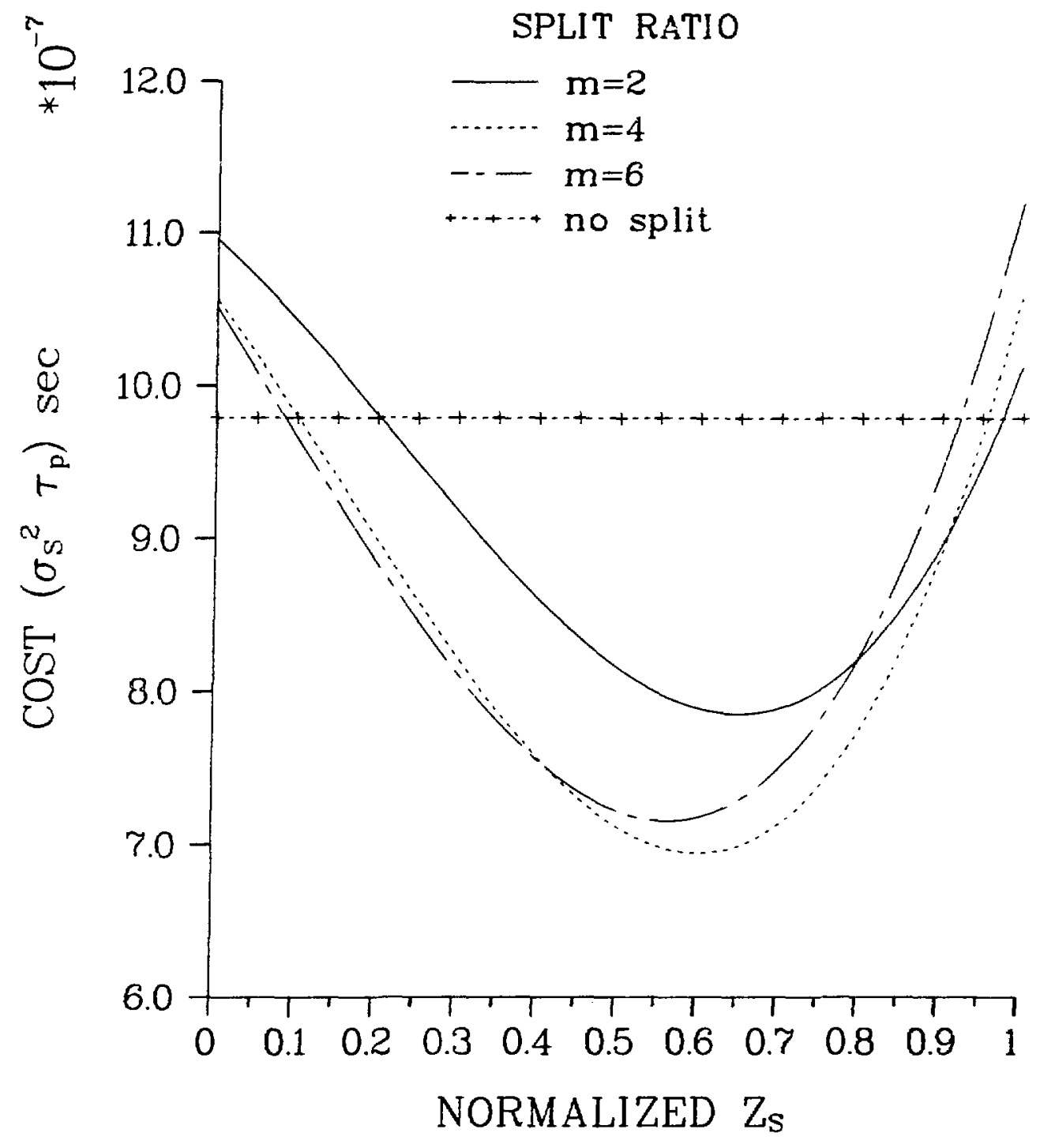

COST VS SPLITTING SURFACE LOCATION $(D=5.00, W s=0.10)$

Figure 23 


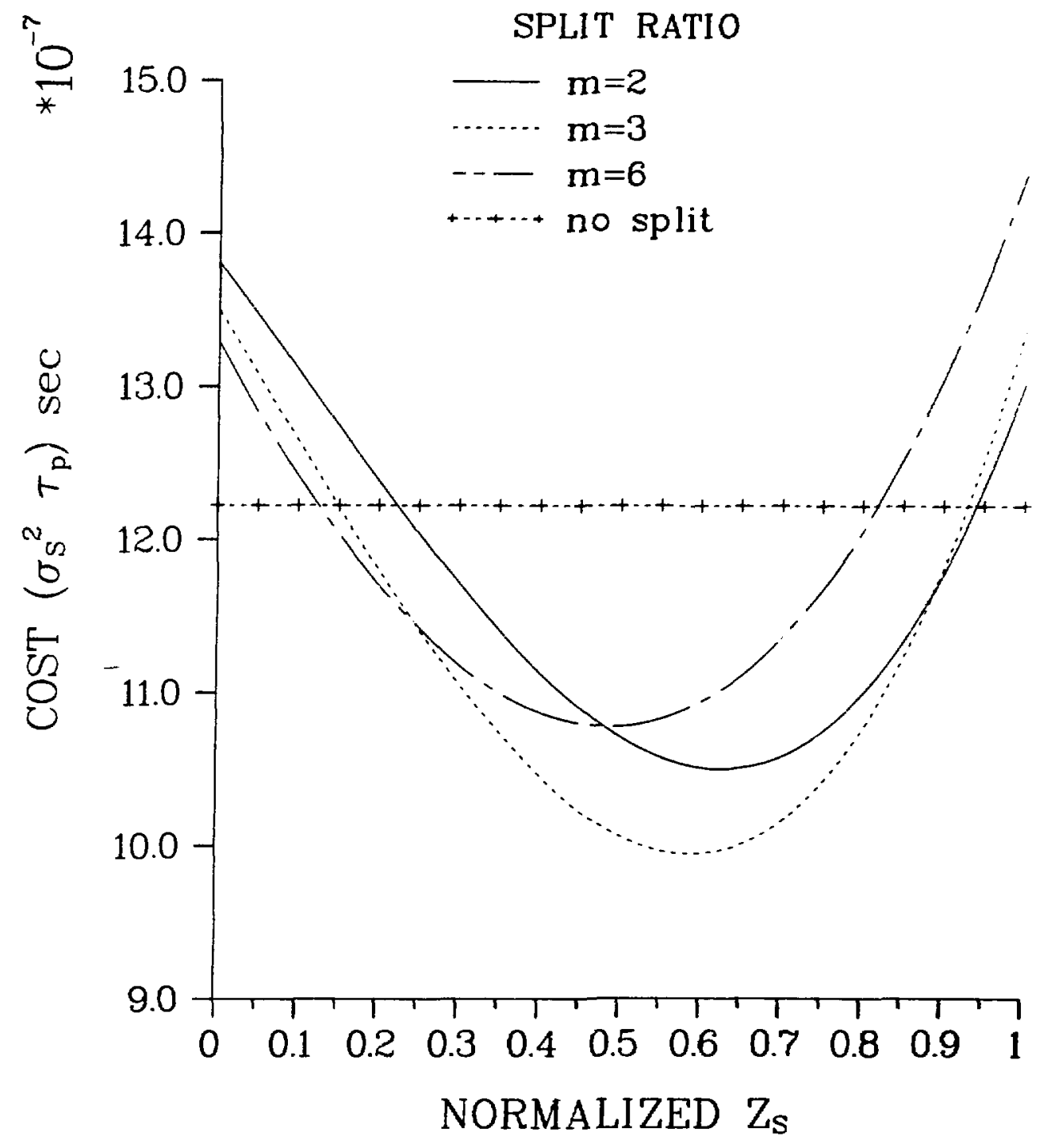

COST vS SPLITTING SURFACE LOCATION $\left(D=5.00, W_{S}=0.50\right)$

Figure 24 


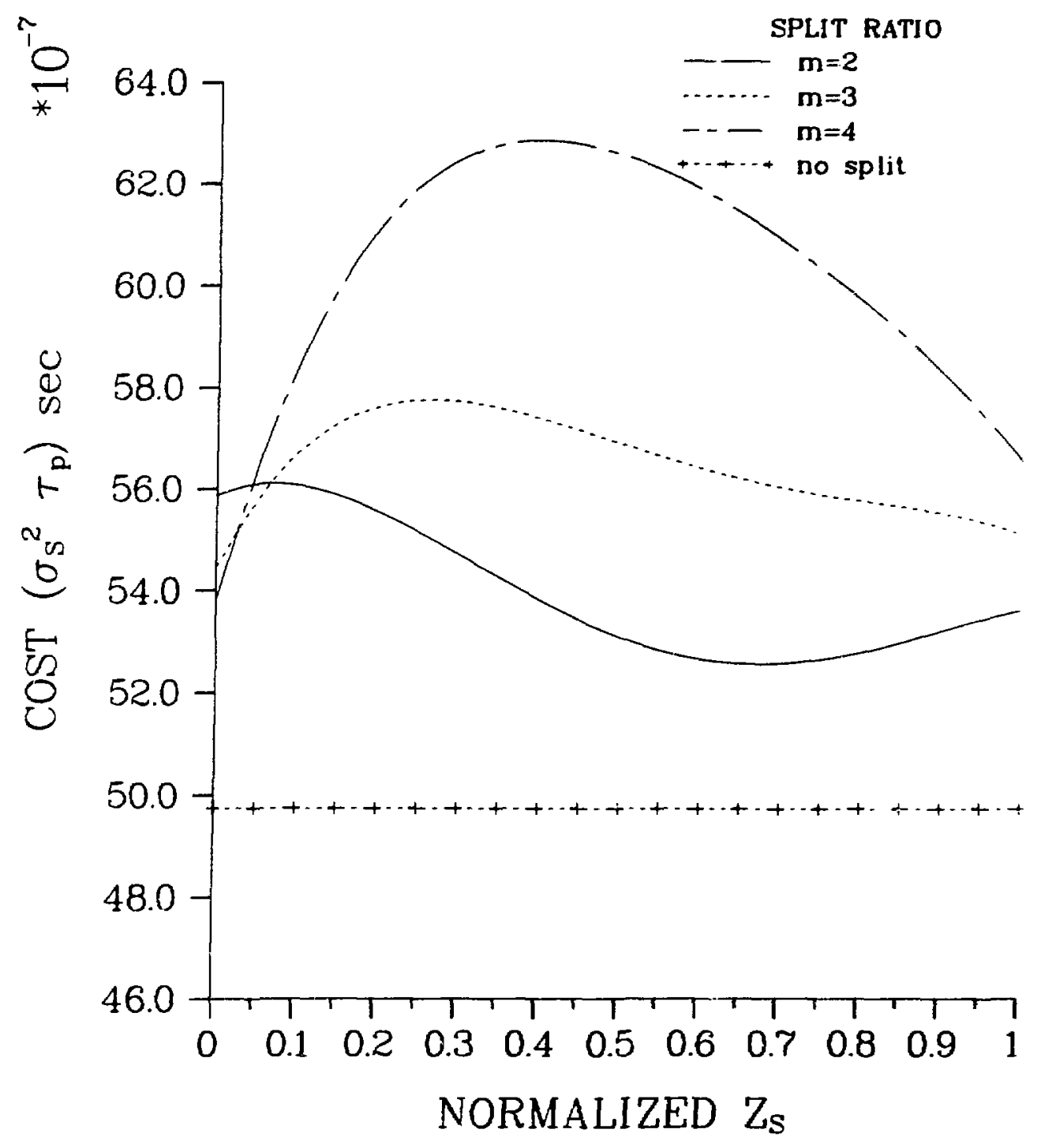

COST vS SPLITTING SURFACE LOCATION $(D=5.00, W s=0.90)$

Figure 25 


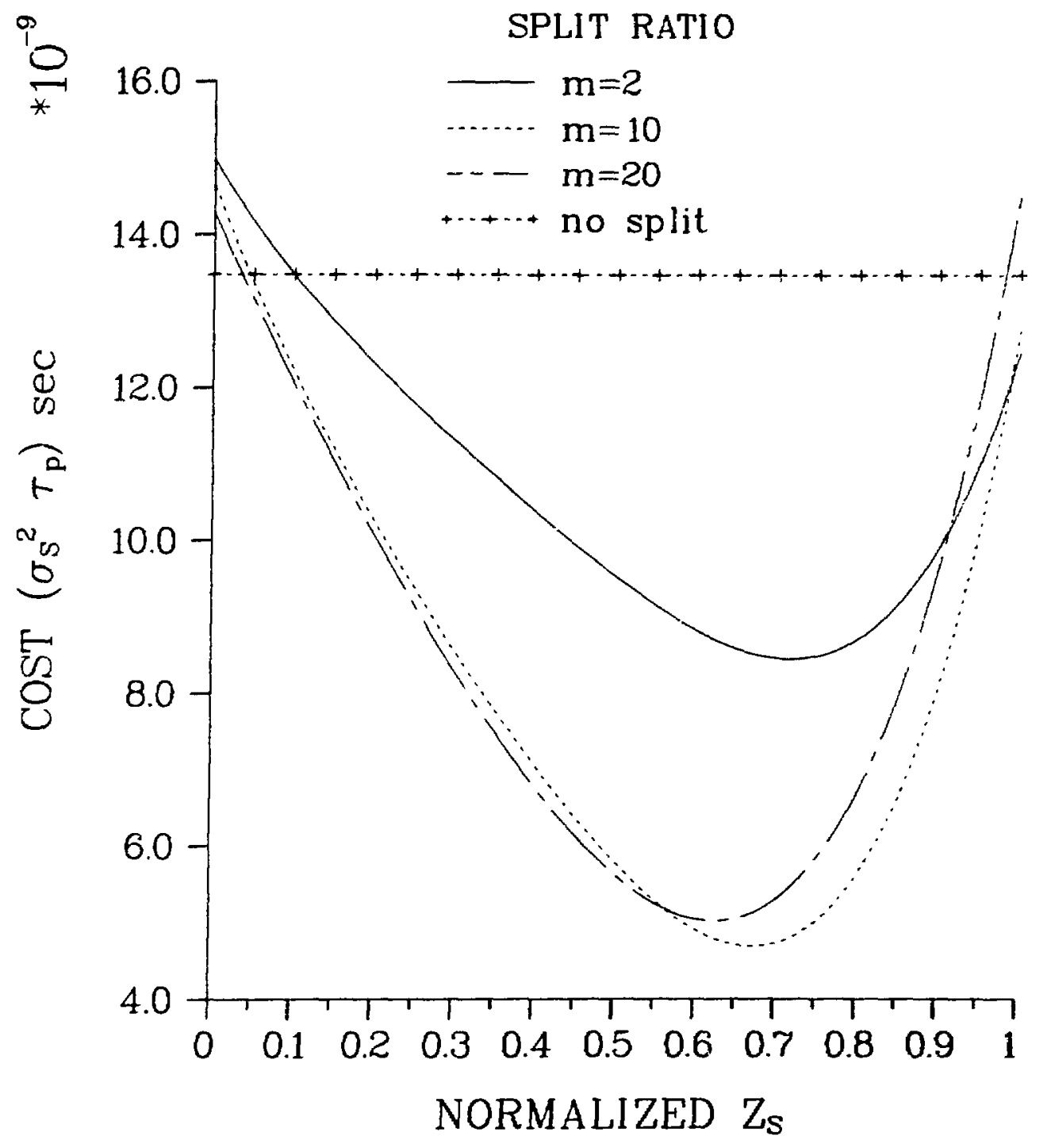

COST vs SPLITTING SURFACE LOCATION
$\left(D=10.0, \mathrm{Ws}_{\mathrm{s}}=0.10\right)$

Figure 26 


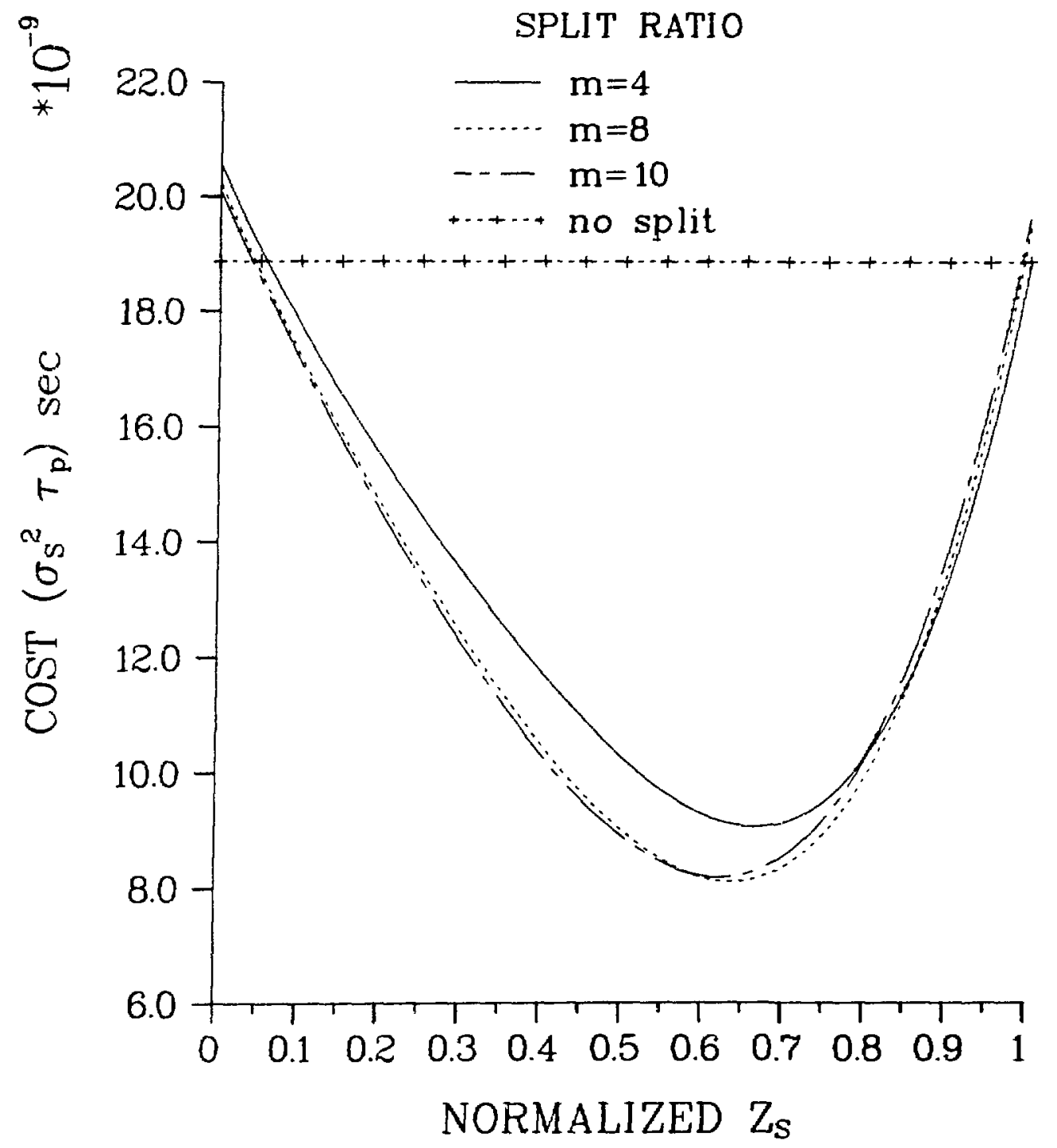

COST vS SPLITTING SURFACE LOCATION $\left(D=10.0, W_{s}=0.50\right)$

Figure 27 


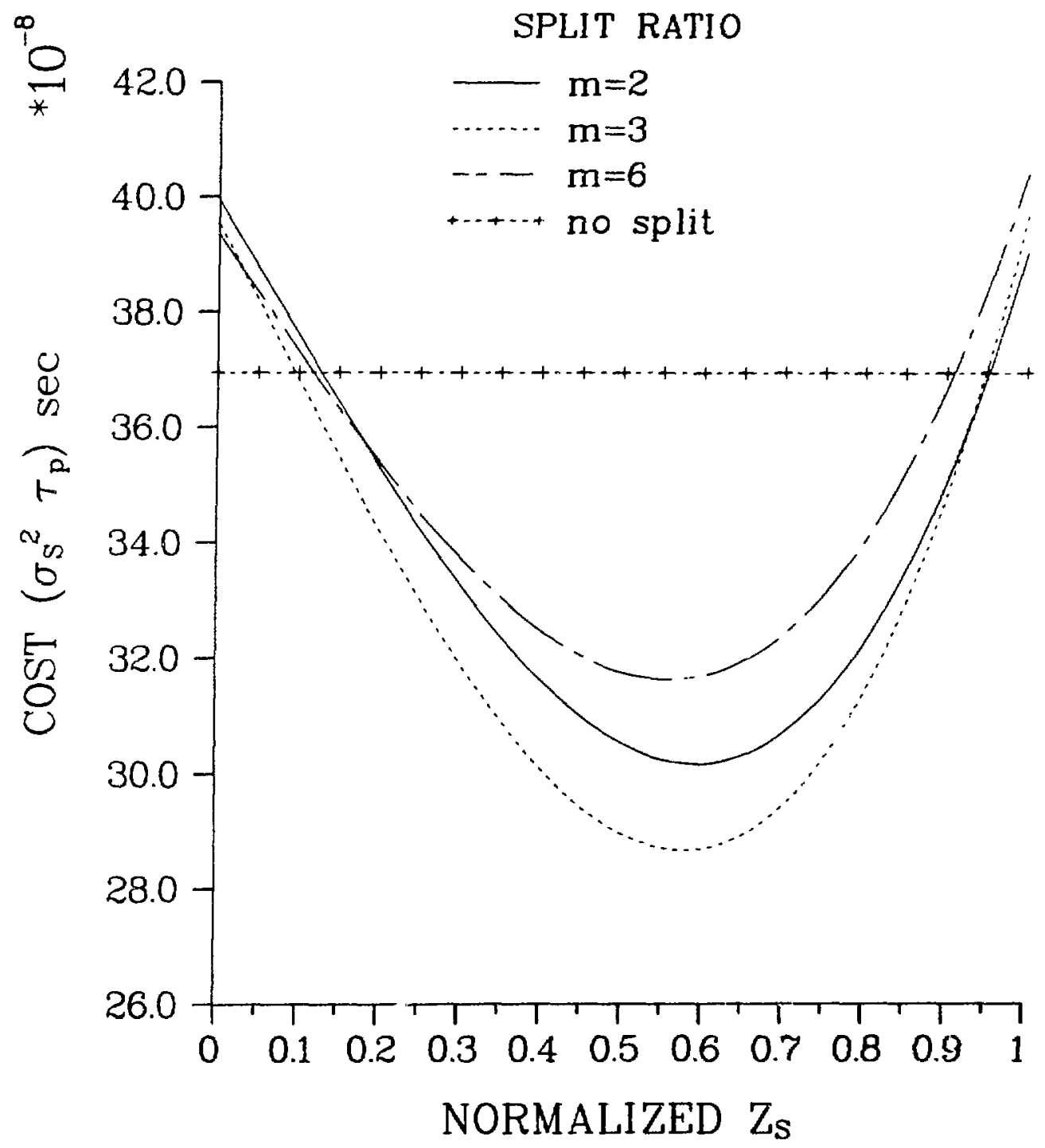

COST vS SPLITTING SURFACE LOCATION $\left(D=10.0, W_{s}=0.90\right)$

Figure 28 
Here splitting is optimized with $m=4$ and with the surface located about $60 \%$ of the way into the slab $(D=5.0 \mathrm{mfp})$.

When the scattering ratio is increased to 0.50 (Fig. 24), splitting is still cost-effective, but the optimum splitting ratio drops to $m=3$. Finally, by increasing the degree of scattering to $\mathrm{w}_{\mathrm{S}}=0.90$, splitting loses its cost-effectiveness vis a' vis non-splitting (Fig. 25).

In the case of a thinner slab $(D=3.0 \mathrm{mfp})$, the cost function might still be minimized, as indicated in Fig. 20; however, even with $90 \%$ absorption, it does not pay to split. In fact, raising the scatterIng ratio for $D=3.0 \mathrm{mf}$ or decreasing the slab thickness even further results in cases where splitting is not cost-effective relative to the non-splitting calculation.

On the other hand, Fig. 26 presents results for a slab thickness of $D=10.0 \mathrm{mfp}$. With $\mathrm{w}_{\mathrm{s}}=0.10$, the optimum splitting ratio is $\mathrm{m}=10$. But note that splitting by as much as a factor of 20 does not result in a significant cost penalty. Finally, for slab thicknesses sufficiently large, even with a scattering ratio of 0.90 , splitting gives a good cost benefit (Fig. 28).

Table 5 summarizes the optimal splitting conditions for the cases studied. The optimum splitting surface location in each case is normalized to the total slab thickness $\left(\bar{z}_{s} \equiv z_{s} / D\right)$. 


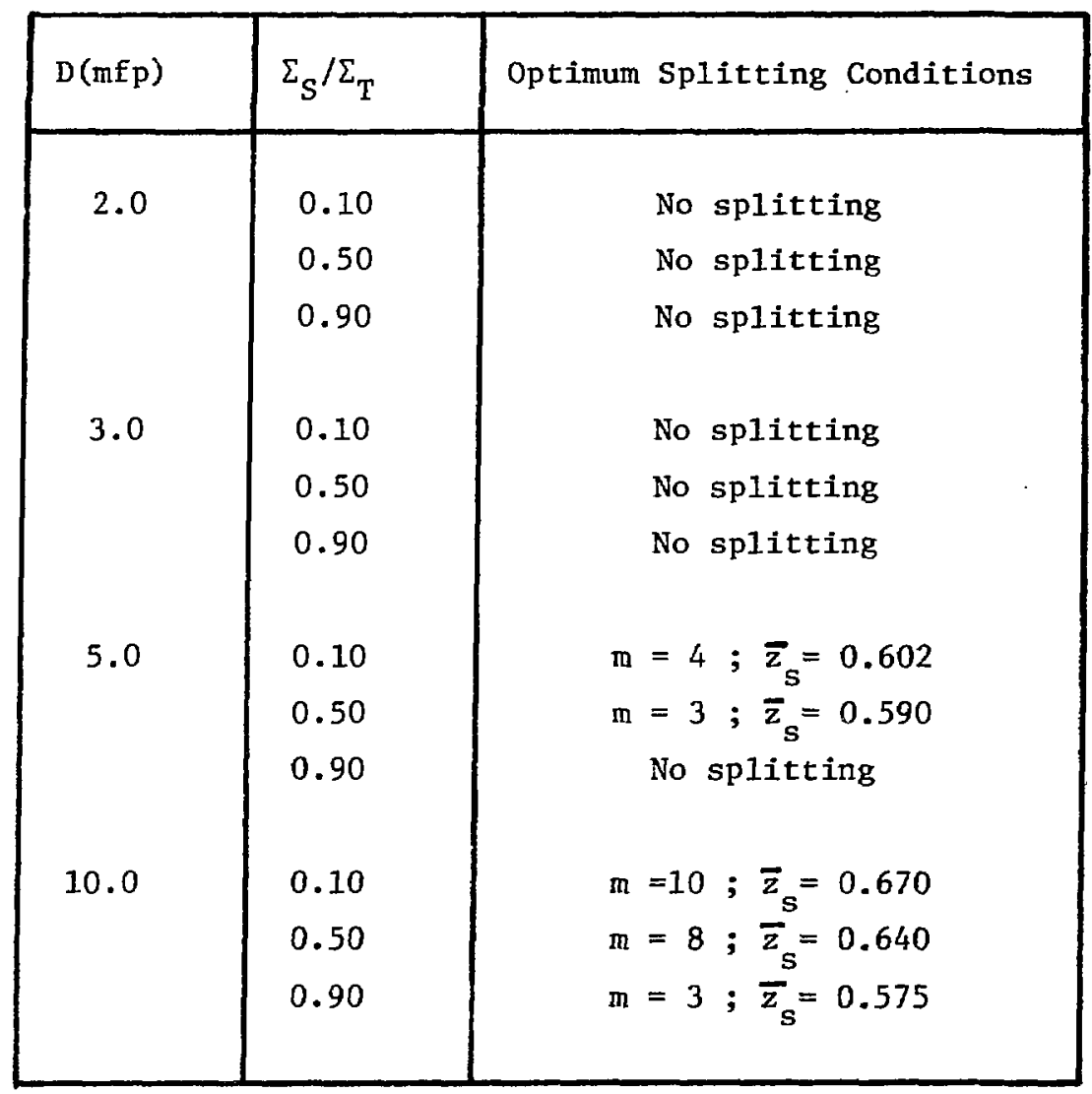

Table 5. Optimal Single-Surface Splitting Conditions 
CHAPTER V

THE CASE OF MULTIPLE SPLITTING SURFACES

Although the bulk of the numerical work of this dissertation is related to a single splitting surface, the material in this chapter is presented in oxder to show that the analysis employed in treating the single surface generalizes quite easily to the case of multiple internal splitting surfaces. Following an outline of the derivations for the $r$-th moment of the transmission score as well as $\tau_{p}$ (which follow very closely the treatment presented in Chapter IV), results of a numerical study involving the optimization of two splitting surfaces in a slab of fixed thickness and scattering ratio are presented. A cost comparison among optimal two-si rface, optimal one-surface, and several heuristic schemes of splitting is also presented. Finally, a comparison is made for several cases between the sample variance predicted deterministically and the stochastic value computed using a standard production Monte Carlo code (the MCNP $\operatorname{code}^{9}$ developed at the Los Alamos Scientific Laboratory).

\subsection{Sample Variance, $\sigma_{s}^{2}$}

The problem under consideration involves $\mathrm{N}$ splitting surfaces which divide the slab into $\mathrm{N}+1$ separate importance regions. This is illustrated in Fig. 29. The formulation of the $r$-th moment equations for $r=1,2$ again proceeds by first formulating the score accumulation probability $\psi$ for the $N+1$ regions and $N$ surfaces of the problem. This, of course, is subject to the particular list of next-events for each case. 


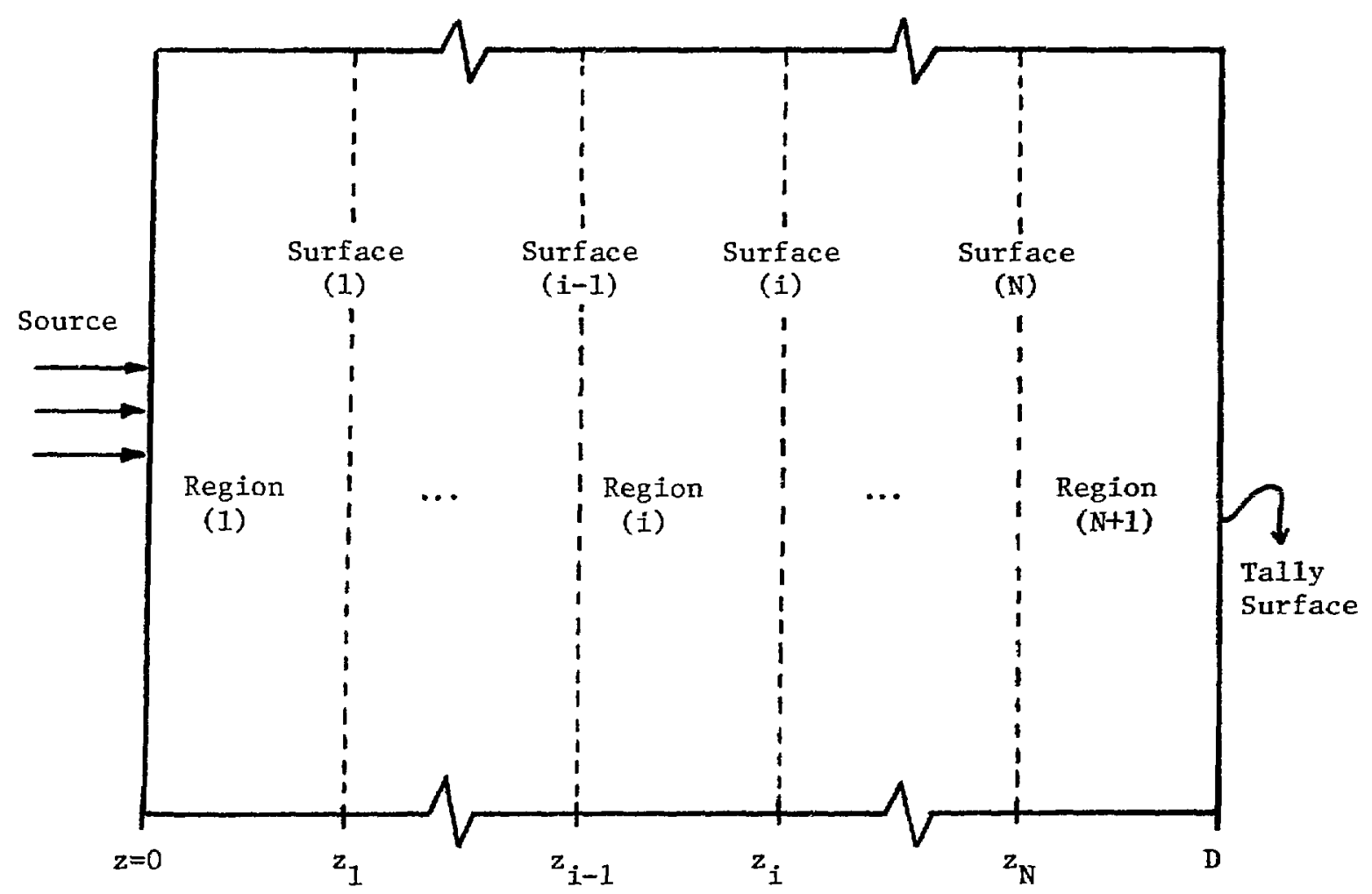

Figure 29. Geometry Involving Multiple Splitting Surfaces. 
Although the multi-surface development follows exactly (in principle) the single-surface derivations, the multiple-surface equations exhibit a greater variety in the formulations due to the different possible groupings of next events for the different regions and surfaces. Due to this added complexity, the reader's interests are best served by offering a somewhat complete treatment which includes all of the possible cases encountered in formulating the multi-surface moment equations. It should be reiterated that the principle and formalism for this derivation follows exactly from the case of a single surface.

Because there are now $N$ internal splitting surfaces $\left(z_{1}, z_{2}, \cdots, z_{N}\right)$, several definitions presented in Chapter IV should be generalized to reflect this condition:

$\mathrm{m}_{\mathrm{i}}$

$\mathrm{p}_{S \mathrm{~m}_{i}}\left(\mathrm{P}^{-}, \mathrm{s}\right) \mathrm{ds}$

$p_{R_{i}}\left(\underline{P}^{-}, s\right) d s$

$S_{m_{i}}\left(z, \underline{P}^{+}\right) d z^{+}$
Splitting ratio at surface $z_{i}$.

Score distribution function for splitting by $m_{i}: 1$ at the $i-t h$ splitting surface ( $\underline{P}^{\prime}$ on the $i-t h$ splitting surface).

Score distribution function for a Russian roulette event at the i-th splitting surface.

$\equiv$ Probability of experiencing an $m_{i}: 1$ splitting event in $\mathrm{dz}^{+}$about $\mathrm{z}^{+}$, given the particle starts at $z$ and reaches $z^{+}$without collision.

$=\left\{\begin{array}{c}s_{m_{i}}\left(z^{+}, \mu^{+}, w^{+}\right) d z^{+}=H\left(\mu^{+}\right) \delta\left(z^{+}-z_{i}\right) d z^{+} \\ \left.\text {for } z_{i-1} \leq z<z_{i} \quad \text { (region } i\right), \\ 0 \text { for } z<z_{i-1} \text { and } z \geq z_{i} .\end{array}\right.$ 


$$
\begin{aligned}
& \mathrm{R}_{\mathrm{m}}\left(\mathrm{z}, \underline{\underline{p}}^{+}\right) \mathrm{d} \mathrm{z}^{+} \\
& \mathrm{D}_{0}\left(\underline{\mathrm{P}}, \underline{\mathrm{P}}^{+}\right) \mathrm{d \underline { \textrm {P } } ^ { + }} \\
& \mathrm{D}_{\mathrm{T}}\left(\underline{\mathrm{P}}, \underline{\mathrm{P}}^{+}\right) \mathrm{d} \underline{\mathrm{P}}^{+} \\
& \mathrm{B}_{\mathrm{Sm}}\left(\underline{\mathrm{P}}^{+}, \underline{\mathrm{P}}^{-\infty}\right) \mathrm{d} \underline{\mathrm{P}}^{-\infty} \\
& \equiv \text { Probability that all }\left(m_{i}\right) \text { sub-particles resulting } \\
& \text { from a splitting event at } \underline{P}^{+} \text {will each exit in } \\
& \underline{\mathrm{dP}}^{\wedge} \text { about } \underline{\mathrm{P}}^{-1} \text {, } \\
& =\delta\left(z^{\prime}-z^{+}\right) \delta\left(\mu^{\prime \prime}-\mu^{+}\right) \delta\left(w^{\prime \prime}-w^{+} / m_{i}\right) d z^{\prime} d \mu^{\prime \prime} d w^{\prime \prime} . \\
& \xi_{\mathrm{m}_{i}} \\
& \text { 三 Probability of experiencing a Russian roulette } \\
& =\left\{\begin{aligned}
R_{\mathrm{m}_{i}}\left(z^{+}, \mu^{+}, w^{+}\right) & =\left[1-\mathrm{H}\left(\mu^{+}\right)\right] \delta\left(z^{+}-z_{i}\right) \mathrm{dz}^{+} \\
\text {for } \quad z_{i} & <z \leq z_{i+1} \quad(\text { region } i+1), \\
0 & \text { for } z \leq z_{i} \text { and } z<z_{i+1}
\end{aligned}\right. \\
& \text { E Probability of escaping the front surface at } \\
& =\left\{\begin{array}{c}
\mathrm{T}\left(z \rightarrow \mathrm{z}^{+}, \mu\right)[1-\mathrm{H}(\mu)] \delta\left(\mu^{+}-\mu\right) \delta\left(w^{+}-w\right) \delta\left(z^{+}\right) \\
\times \mathrm{dz} \mathrm{d}^{+} \mu^{+} \mathrm{d} w^{+} \\
\text {for } z \leq z_{1} \quad \text { (region 1) } \\
\text { for } z>z_{1}
\end{array}\right. \\
& =\left\{\begin{array}{c}
T\left(z \rightarrow z^{+}, \mu\right) H(\mu) \delta\left(\mu^{+}-\mu\right) \delta\left(w^{+}-w\right) \delta\left(z^{+}-D\right) \\
\times d z^{+} d \mu^{+} d w^{+} \\
\text {for } z \geq z_{N} \quad(\text { region } N+1) \\
\text { for } z<z_{N} .
\end{array}\right. \\
& \equiv \text { Russian roulette survival probability at the i-th } \\
& =1 / \mathrm{m}_{i} \text {. }
\end{aligned}
$$




$$
\begin{aligned}
& { }^{\mathrm{B}} \mathrm{m}_{\mathrm{i}}\left(\xi_{\mathrm{m}_{i}} ; \underline{\mathrm{P}}^{+}, \underline{\mathrm{P}}^{\prime \prime}\right) \mathrm{dP}^{\prime \prime} \equiv \begin{array}{l}
\text { Probability that a particle undergoing a Russian } \\
\text { roulette event at } \mathrm{p}^{+} \text {will leave the event in }
\end{array} \\
& \text { d } \underline{p}^{\prime} \text { about } \underline{P}^{\prime \prime} \text {, given the survival probability } \\
& \text { is } \xi_{\mathrm{m}_{i}} \text {, } \\
& =\delta\left(z^{\prime \prime}-z^{+}\right) \delta\left(\mu^{\prime \prime}-\mu^{+}\right)\left\{\xi_{m_{i}} \delta\left(w^{\prime \prime}-w^{+} / \xi_{m_{i}}\right)\right. \\
& \left.+\left(1-\xi_{\mathrm{m}_{\mathrm{i}}}\right) \delta\left(\mathrm{w}^{\prime \prime}\right)\right) \mathrm{d} \mathrm{z}^{\prime}{ }^{\prime} \mathrm{d} \mu{ }^{-} \mathrm{dw}^{\prime \prime} \\
& =\beta_{0 m_{i}}\left(\underline{p}^{+}, \underline{p}^{\prime}\right) d \underline{p}^{\prime}+\left(1-\xi_{m_{i}}\right) \delta\left(z^{-}-z^{+}\right) \delta\left(\mu^{-}-\mu^{+}\right) \\
& \times \delta\left(\mathrm{w}^{\prime \prime}\right) \mathrm{dz} \mathrm{z}^{\prime} \mathrm{d} \mu \mu^{\prime \prime} \mathrm{dw} \mathrm{w}^{\prime \prime} .
\end{aligned}
$$

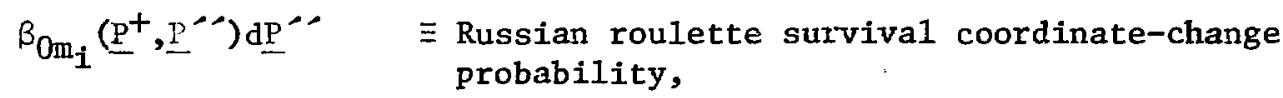

$$
\begin{aligned}
& =\xi_{\mathrm{m}_{i}} \delta\left(z^{-\prime}-z^{+}\right) \delta\left(\mu^{\prime}-\mu^{+}\right) \delta\left(w^{\prime}-w^{+} / \xi_{m_{i}}\right) \\
& \times \mathrm{dz}^{\prime} \mathrm{d} \mathrm{d}{ }^{-} \mathrm{d} \mathrm{w}^{-\infty} .
\end{aligned}
$$

As discussed in Chapter IV, the formulation of the total score accumulation probability proceeds by first considering the possible next events following the departure of a particle from point $\underline{P}$ in phase space. Here six cases are considered for particles beginning their next flight: in region 1 , on surface 1 , in an internal region bounded by two splitting surfaces (region $i$ ), on an internal surface separating two internal regions (surface $i$ ), on surface $N$, and in region $N+1$. The next events for each of these cases are listed below:

Region $1\left(0 \leq z<z_{1}\right)$; see Fig. 30a

- Scattaring collision in region 1

- Front silface escape

- Splitting $\left(\mathrm{m}_{1}: 1\right)$ at $z=z_{1}$ 
Surface $1\left(z=z_{1}\right)$; see Fig. $30 \mathrm{~b}$

- Scattering collision in region 1

- Scattering collision in region 2

- Front-surface escape

-Splitting $\left(\mathrm{m}_{2}: 1\right)$ at $z=z_{2}$.

Region $i\left(z_{i-1}<z<z_{i}\right)$; see Fig. 31a

- Scattering collision in region i

- Russian roulette at $z=z_{i-1}$

- Splitting $\left(m_{i}: 1\right)$ at $z=z_{i}$

Surface $i\left(z=z_{i}\right.$, where $\left.1<i<N\right)$; see Fig. $31 b$

- Scattering collision in region $i$

- Scattering collision in region $i+1$

- Russian roulette at $z=z_{i-1}$

- Splitting $\left(m_{i+1}: 1\right)$ at $z=z_{i+1}$

Surface N $\left(z=z_{N}\right)$; see Fig. 32a

- Scattering collision in region $\mathbb{N}$

- Scattering collision in region $N+1$

- Russian roulette at $z=z_{N-1}$

Region $N+1\left(z_{N}<z \leq D\right)$; see Fig. 32b

- Scattering collision in region $N+1$

- Russian roulette at $z=z_{N}$

- Back-surface escape at $z=D$

For each of the cases 1isted above, the total score accumulation probability may be formulated. The notation $\psi_{1}(\underline{P}, s)$ ds will represent the score accumulation probability for particle starting locations in region $i ; \psi_{z_{i}}(\underline{P}, s) \mathrm{ds}$ will represent the probability for particles with starting location on the i-th splitting surface. The formulation of the score accumulation probability for each of these cases follows along the lines outlined in Chapter IV, i.e., in developing Eqs. (4.1.1.1) 


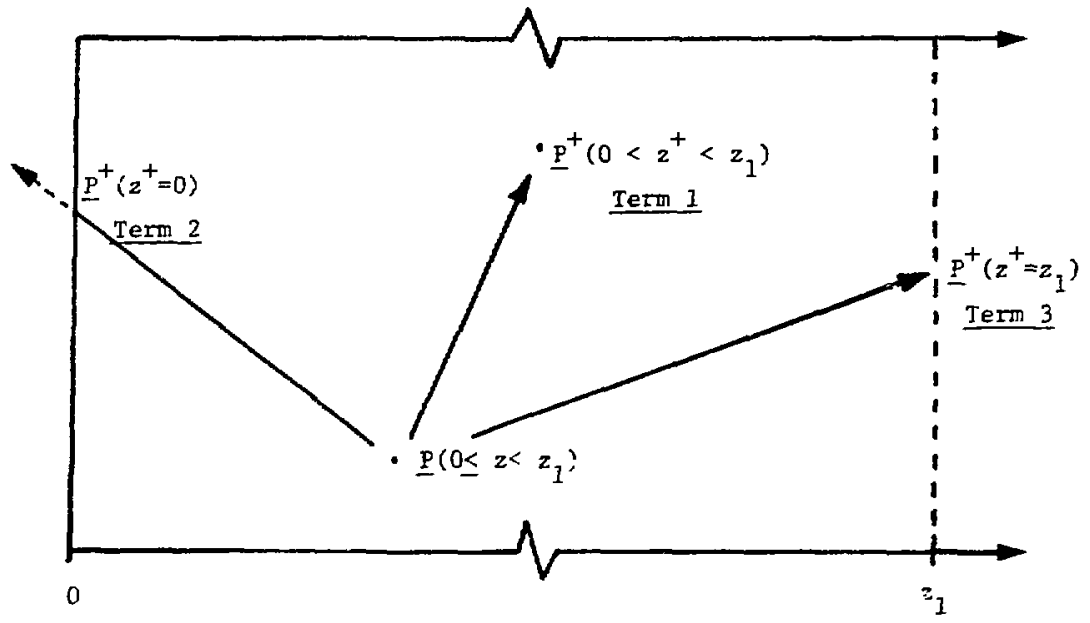

Figure 30a. Next Events: Particle Starts in Region $1,0 \leq z<z_{1}$. [References to Texms of Eq.(5.1.1).]

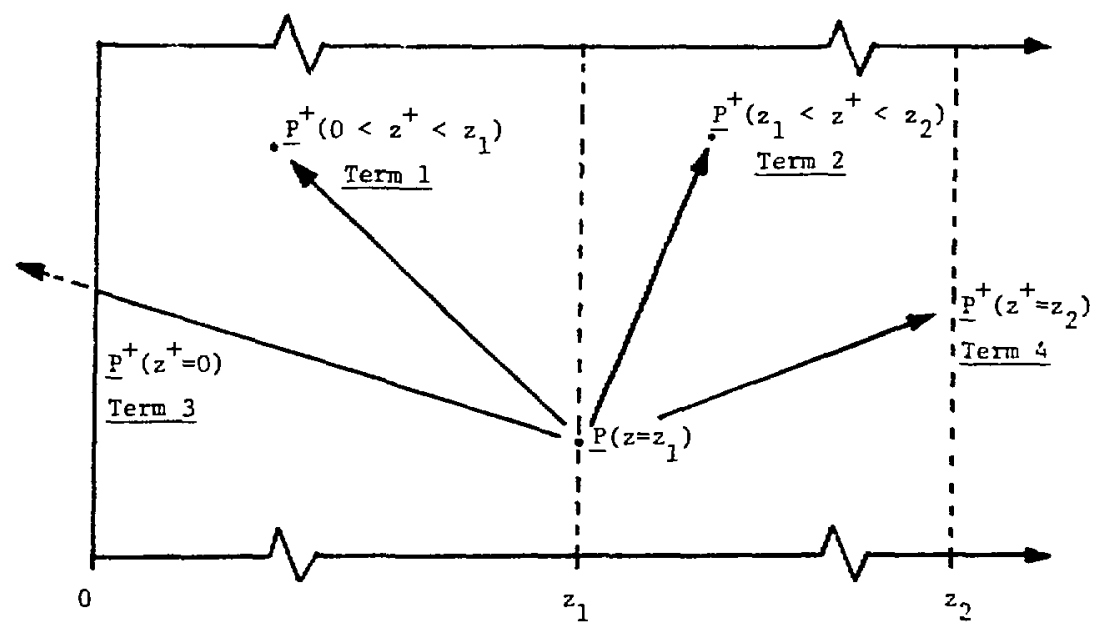

Figure $30 \mathrm{~b}$. Next Events: Particle Scarts at $z=2$.

[References to Terms of Eq.(5.1.2).] 


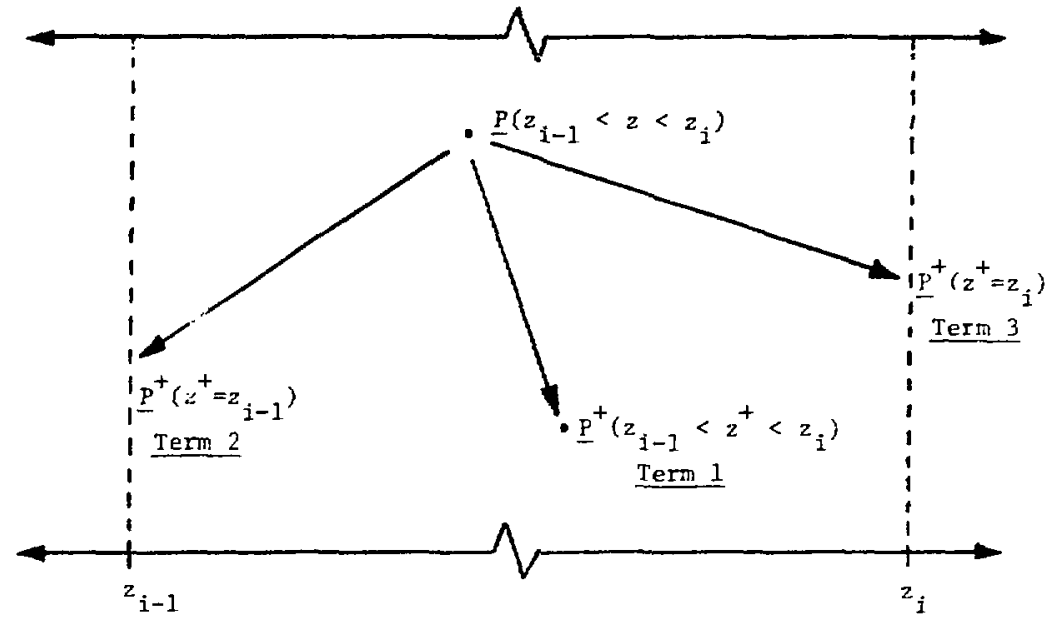

Figure 31a. Next Events: Particle Starts in Region i, $z_{i-1}<z<z_{i}$. [References to Terms of Eq. (5.1.3).]

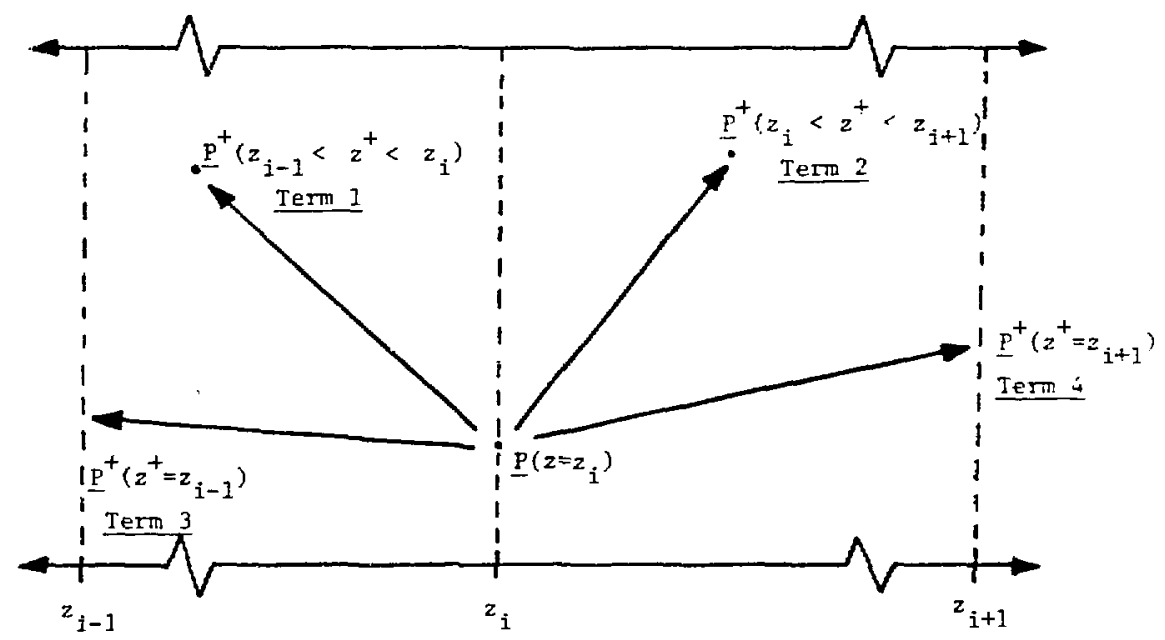

Figure 31b. Next Events: Particle Starts at $z=z_{i}$.

[References to Terms of Eq. (5.1.4).] 


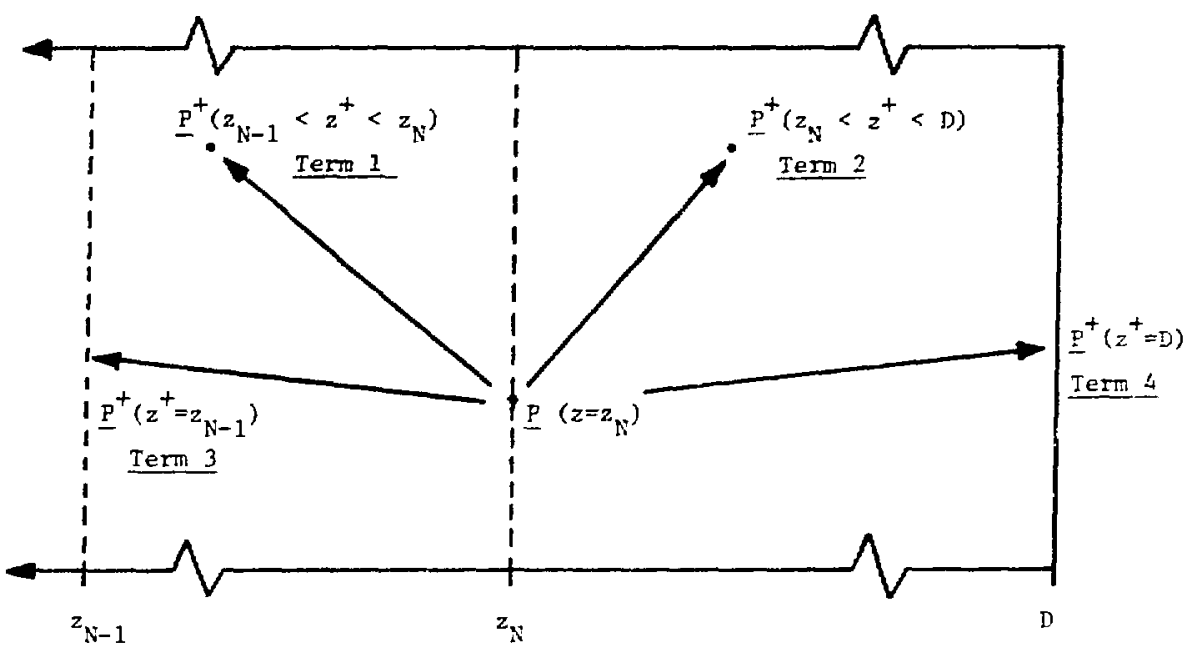

Figure 32a. Next Events: Particle Starts at $z=z$ N

[References tc Terms of Eq.(5.1.5).]

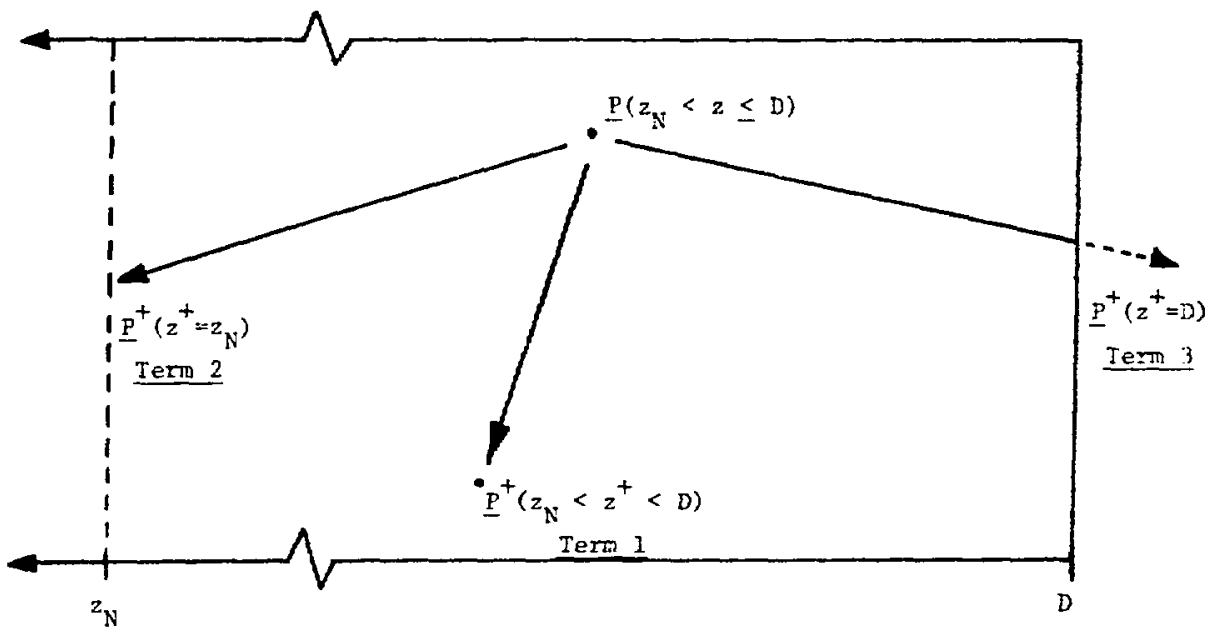

Figure 32b. Next Events: Particle Starts in Region $N+1$,

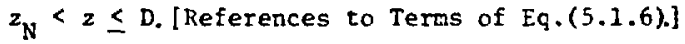


through (4.1.1.3). In each case, a separate term accounts for one of the next events as listed above (see also Figs. 30 through 32). The equations are presented here, given the particle starts out at $\underline{\underline{p}}$, with $z$.

In region $1\left(0 \leq z<z_{1}\right)$ :

$$
\begin{aligned}
& \psi_{1}(\underline{P}, s) \mathrm{ds}=\int_{0<z^{+}<z_{I}} \underline{\mathrm{P}}^{+} \mathrm{T}\left(\underline{\mathrm{P}}, \underline{\mathrm{P}}^{+}\right) \int \underline{\mathrm{P}}^{-} \sigma\left(\underline{\mathrm{P}}^{+}, \underline{\mathrm{p}}^{\prime}\right) \int \mathrm{ds^{- }} \mathrm{p}_{\mathrm{S}}\left(\underline{\mathrm{P}}^{-}, \mathrm{s}^{\prime}\right) \int \underline{\mathrm{P}}^{-} \\
& \left.\times\left[E\left(\underline{\mathrm{P}}^{-}, \underline{\mathrm{P}}^{-\prime}\right) \psi_{1}\left(\underline{\mathrm{P}}^{-}, s-\mathrm{s}^{\prime}\right)\right] \mathrm{d} s+\int \mathrm{d \underline {P } ^ { + }} \mathrm{D}_{0}\left(\underline{\mathrm{P}}, \underline{\mathrm{P}}^{+}\right) \int \mathrm{d} s^{\prime} \mathrm{p}_{\mathrm{E}, 0} \underline{\mathrm{P}}^{+}, \mathrm{s}^{\prime}\right) \\
& \left.\times \delta\left(s-s^{\prime}\right) d s+\int d \underline{P}^{+} T\left(\underline{P}, \underline{P}^{+}\right) s_{m_{1}} \underline{P}^{+}\right) \int d s^{\prime} p_{S_{m_{1}}}\left(\underline{P}^{+}, s^{\prime}\right) \int d \underline{P}^{-} \\
& \times \mathrm{B}_{\mathrm{Sm}_{1}}\left(\underline{\mathrm{P}}^{+}, \underline{\mathrm{P}}^{-\cdots}\right) \int \mathrm{ds_{1 }} \cdots \int \mathrm{ds}_{\mathrm{m}_{1}-1} \psi_{\mathrm{z}_{1}}\left(\underline{\mathrm{P}}^{-}, \mathrm{s}_{1}\right) \cdots \psi_{\mathrm{z}_{1}}\left(\underline{\mathrm{P}}^{\cdots}, \mathrm{s}_{\mathrm{m}_{1}-1}\right) \\
& \times \psi_{z_{1}}\left(\underline{P}^{\prime}, s-\left(s^{\prime}+s_{1}+s_{2}+\cdots+s_{\mathrm{n}_{1}-1}\right)\right) \mathrm{ds} .
\end{aligned}
$$

On surface $1\left(z=z_{1}\right)$ : 


$$
\begin{aligned}
& \psi_{z_{1}}(\underline{P}, s) d s=\int_{0<z^{+}<z_{1}} d \underline{P}^{+} T\left(\underline{P}, \underline{P}^{+}\right) \int d \underline{P}^{\prime} \sigma\left(\underline{P}^{+}, \underline{P}^{\prime}\right) \int d s^{\prime} p_{s}\left(\underline{P}^{\prime}, s^{\prime}\right) \int d \underline{P}^{-} \\
& \times\left[E\left(\underline{P}^{\prime}, \underline{P}^{-}\right) \psi_{1}\left(\underline{P}^{-}, s-s^{\prime}\right)\right] d s+\int_{z_{1}<z^{+}<z_{2}} \underline{d P}^{+} T\left(\underline{P}, \underline{\underline{P}}^{+}\right) \int d \underline{P}^{\prime} \\
& \times \sigma\left(\underline{P}^{+}, \underline{P}^{\prime}\right) \int d s^{\prime} p_{S}\left(\underline{P}^{\prime}, s^{\prime}\right) \int d \underline{P}^{\prime} E\left(\underline{P}^{\prime}, \underline{P}^{\prime}\right) \psi_{2}\left(\underline{P}^{\prime}, s-s^{\prime}\right) d s \\
& +\int d \underline{P}^{+} D_{0}\left(\underline{P}, \underline{P}^{+}\right) \int d s^{\prime} \mathrm{P}_{E, 0}\left(\underline{P}^{+}, s^{\prime}\right) \delta\left(s-s^{\prime}\right) d s+\int d \underline{P}^{+} \mathrm{T}\left(\underline{P}, \underline{P}^{+}\right)
\end{aligned}
$$

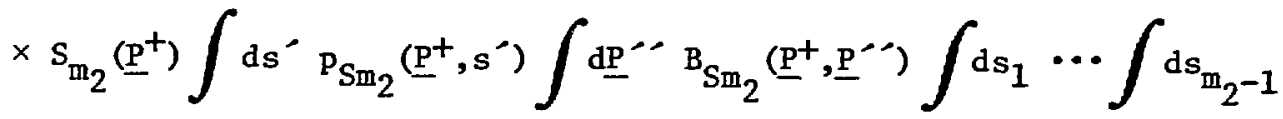

$$
\begin{aligned}
& \times \psi_{z_{2}}\left(\mathrm{P}^{\cdots}, \mathrm{s}_{1}\right) \cdots \psi_{z_{2}}\left(\underline{\mathrm{P}}^{-}, \mathrm{s}_{\mathrm{m}_{2}-1}\right) \\
& \times \psi_{z_{2}}\left(\underline{P}^{\prime}, s-\left(s^{\prime}+s_{1}+\cdots+s_{m_{2}-1}\right)\right) d s .
\end{aligned}
$$

In region $i\left(z_{i-1}<z<z_{i}\right.$, with $\left.1<i<N\right)$ :

$$
\begin{aligned}
& \psi_{j .}(\underline{P}, s) d s=\int_{z_{i-1}<z^{+}<z_{i}} d \underline{P}^{+} T\left(\underline{P}, \underline{P}^{+}\right) \int d \underline{P}^{-} \sigma\left(\underline{P}^{+}, \underline{P}^{\prime}\right) \int d s^{-} p\left(\underline{P}^{-}, \underline{s}^{\prime}\right) \int d \underline{P}^{-} \\
& \times\left[E\left(\underline{P}^{\prime}, \underline{P}^{\prime}\right) \psi_{i}\left(\underline{P}^{\prime}, s-s^{\prime}\right)\right] d s+\int d \underline{P}^{+} T\left(\underline{P}, \underline{P}^{+}\right) R_{m_{i-1}}\left(\underline{P}^{+}\right) \int d s^{\prime} \\
& \times\left[p_{R_{i-1}}\left(\underline{P}^{+}, s^{\prime}\right) \int d \underline{P}^{\prime \prime} B_{0 m_{i-1}}\left(\xi_{m_{i-1}} ; \underline{P}^{+}, \underline{P}^{\prime \prime}\right) \psi_{z_{i-1}}\left(\underline{P}^{-\prime}, s-s^{\prime}\right)\right] d s \\
& +\int \mathrm{d} \underline{P}^{+} \mathrm{T}\left(\underline{\mathrm{P}}, \underline{\mathrm{P}}^{+}\right) \mathrm{s}_{\mathrm{m}_{1}}\left(\underline{\mathrm{P}}^{+}\right) \int \mathrm{d} \mathrm{s}^{-} \mathrm{P}_{\mathrm{Sm}_{\mathrm{i}}}\left(\underline{\mathrm{P}}^{+}, \mathrm{s}^{\prime}\right) \int \mathrm{d} \underline{\mathrm{P}}^{-\infty} \mathrm{B}_{\mathrm{Sm}_{1}}\left(\underline{\mathrm{P}}^{+}, \underline{\mathrm{P}}^{-}\right) \\
& \times\left[\int d s_{1} \cdots \int d s_{m_{i}-1} \psi_{z_{i}}\left(\underline{P}^{-\cdots}, s_{1}\right) \cdots \psi_{z_{i}}\left(\underline{P}^{\mu}, s_{m_{1}-1}\right)\right. \\
& \left.\times \psi_{z_{1}}\left(\underline{P}^{\rho \wedge}, s-\left(s^{\wedge}+s_{1}+\cdots+s_{m_{1}-1}\right)\right)\right] d s \quad .
\end{aligned}
$$


On surface $i\left(z=z_{i}\right.$, with $\left.1<i<N\right)$ :

$$
\begin{aligned}
& \psi_{z_{1}}(\underline{P}, s) d s=\int_{z_{i-1}<z^{+<z_{i}}} d \underline{P}^{+} T\left(\underline{P}, \underline{P}^{+}\right) \int \underline{P}^{-} \sigma\left(\underline{P}^{+}, \underline{P}^{-}\right) \int d s^{-} P_{S}\left(\underline{P}^{-}, s^{\prime}\right) \int d P^{-}
\end{aligned}
$$

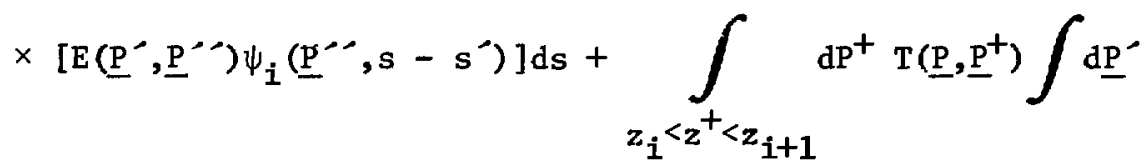

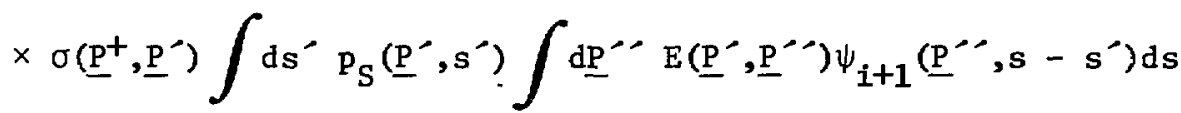

$$
\begin{aligned}
& +\int \underline{\mathrm{P}}^{+} \mathrm{T}\left(\underline{\mathrm{P}}, \underline{\mathrm{P}}^{+}\right) \mathrm{R}_{\mathrm{m}_{\mathrm{i}-1}}\left(\underline{\mathrm{P}}^{+}\right) \int \mathrm{ds^{- }} \mathrm{p}_{\mathrm{R}_{\mathrm{i}-1}}\left(\underline{\mathrm{P}}^{+}, \mathrm{s}^{-}\right) \int \mathrm{dP}^{-}
\end{aligned}
$$

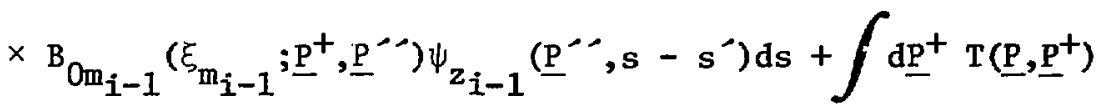

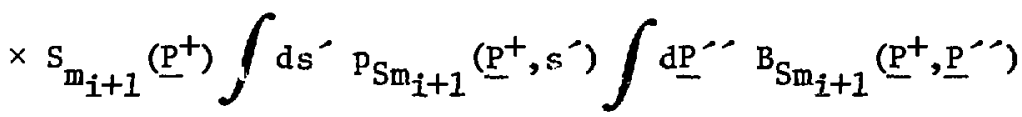

$$
\begin{aligned}
& \times\left[\int d s_{1} \cdots \int d s_{m_{i+1}-1} \psi_{z_{i+1}}\left(\underline{P}^{-}, s_{1}\right) \cdots\right. \\
& \left.\times \psi_{z_{i+1}}\left(\underline{P}^{-\prime}, s-\left(s^{-}+s_{1}+\cdots+s_{m_{i+1}-1}\right)\right)\right] d s .
\end{aligned}
$$

144 


$$
\begin{aligned}
& \text { On surface } N\left(z=z_{N}\right):
\end{aligned}
$$

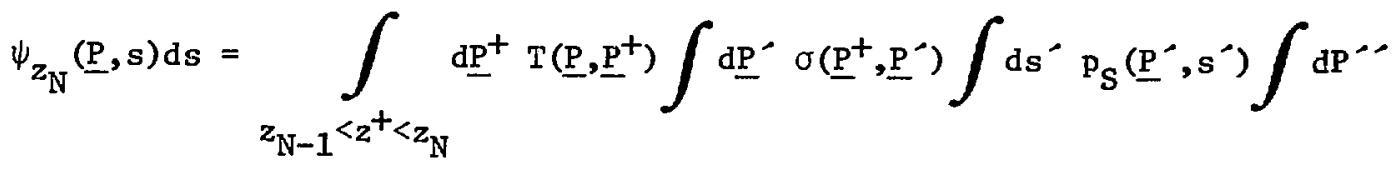

$$
\begin{aligned}
& \times\left[E\left(\underline{P}^{\prime}, \underline{P}^{\prime}\right) \psi_{N}\left(\underline{P}^{\prime-}, s-s^{\prime}\right)\right] d s+\int_{z_{N} z^{+}<D} d_{\underline{P}^{+}} T\left(\underline{P}, \underline{P}^{+}\right) \int d \underline{P}^{\prime} \sigma\left(\underline{\underline{P}}^{+}, \underline{\underline{P}}^{\prime}\right) \\
& \times \int d s^{\prime} p_{S}\left(\underline{P}^{\prime}, s^{\prime}\right) \int d \underline{P}^{-} E\left(\underline{P}^{\prime}, \underline{P}^{\prime}\right) \psi_{N+1}\left(\underline{P}^{-}, s-s^{\prime}\right) d s
\end{aligned}
$$

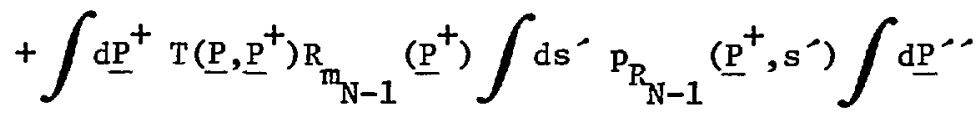

$$
\begin{aligned}
& \left.\times \mathrm{B}_{0 \mathrm{~m}-1}\left(\xi_{\mathrm{m}_{\mathrm{N}-1}} ; \underline{\mathrm{P}}^{+}, \underline{\mathrm{P}}^{-}\right) \psi_{\mathrm{z}_{\mathrm{N}-1}} \underline{(\mathrm{P}}^{-}, \mathrm{s}-\mathrm{s}^{\prime}\right) \mathrm{ds}+\int \mathrm{dP}^{+} \mathrm{D}_{\mathrm{T}}\left(\underline{\mathrm{P}}, \underline{\mathrm{P}}^{+}\right) \int \mathrm{d} \mathrm{s}^{-} \\
& \times p_{E, D}\left(\underline{P}^{+}, s^{\prime}\right) \delta\left(s-s^{\prime}\right) d s .
\end{aligned}
$$

In region $\mathrm{N}+1\left(\mathrm{z}_{\mathrm{N}}<\mathrm{z} \leq \mathrm{D}\right)$ :

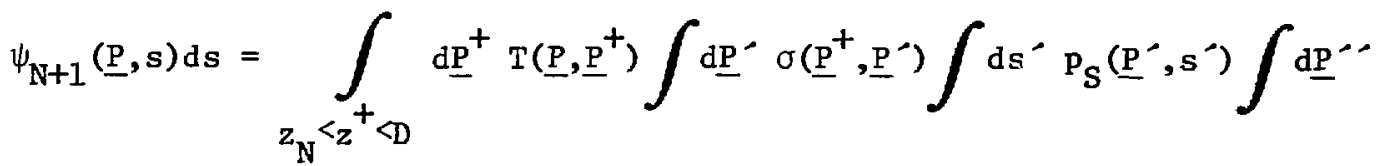

$$
\begin{aligned}
& \left.\times\left[E\left(\underline{P}^{-}, \underline{P}^{-\rho}\right) \psi_{N+1} \underline{P}^{-\rho}, s-s^{\prime}\right)\right] d s+\int \underline{d P}^{+} T\left(\underline{P}, \underline{P}^{+}\right) R_{m_{N}}\left(\underline{P}^{+}\right) \\
& \times \int d s^{\prime} p_{R_{N}}\left(\underline{P}^{+}, s^{\prime}\right) \int d \underline{P}^{\prime}{ }^{B_{0 m_{N}}}\left(\xi_{m_{N}} ; \underline{P}^{+}, \underline{P}^{\prime \prime}\right) \psi_{z_{N}}\left(\underline{P}^{\prime}, s-s^{\prime}\right) d s \\
& +\int \underline{\mathrm{P}}^{+} \mathrm{D}_{\mathrm{T}}\left(\underline{\mathrm{P}}, \underline{\mathrm{P}}^{+}\right) \int \mathrm{ds^{- }} \mathrm{p}_{\mathrm{E}, \mathrm{D}}\left(\underline{\mathrm{P}}^{+}, \mathrm{s}^{-}\right) \delta\left(s-\mathrm{s}^{-}\right) \mathrm{ds} .
\end{aligned}
$$

Given the total score accumulation probabilitles [Eqs. (5.1.1) through $(5.1 .6)]$, it is possible to derive the appropriate equations 
for the $r$-th moment $(r=1,2)$ of the slab transmission score by following the same steps as detailed in Chapter IV. Moreover, these integral equations may be converted to an integro-differential form in order to facilitate solution. A more detailed discussion of the derivation is contained in Appendix $\mathrm{H}$.

The equations are presented here in final form. In fact, the form of the integro-differential moment equations is identical to that determined when considering only a single splitting surface. The following first moment $(r=1)$ formulation, which is derived in Appendix $H$ [Eq. (H.1.9)], applies for all starting locations $z(0 \leq z \leq D)$ :

$$
\begin{aligned}
& -\mu \frac{\partial}{\partial z} F_{1}(z, \mu, w)+\Sigma_{T} F_{1}(z, \mu, w)=\int d \mu^{-} \Sigma_{s} \cdot E\left(z, \mu \rightarrow \mu^{-}\right) F_{1}\left(z, \mu^{-}, w\right) \\
& +H(\mu) \delta(z-D) \frac{\mu}{\Sigma_{T}} \cdot w,
\end{aligned}
$$

where

$$
\mathrm{F}_{1}(\mathrm{z}, \mu, \mathrm{w}) \equiv 1 / \Sigma_{\mathrm{T}} \cdot \mathrm{M}_{1}(\mathrm{z}, \mu, \mathrm{w})
$$

Equation (5.1.7) is identical to Eq. (4.1.2.14), which was derived for the case of one splitting surface. Since splitting and Russian roulette have no effect on the expected value of the Monte Carlo tally, it is expected that the first moment equations be identical. The boundary conditions under which Eq. (5.1.7) is solved are identical to those given in Eqs. (4.1.2.16) and (4.1.2.17), namely

$$
F_{I}(D, \mu>0,1)=I / \Sigma_{T}
$$

and

$$
F_{1}(0, \mu<0,1)=0 .
$$


The following equation for the second moment (see Appendix $\mathrm{H}$ ) holds for all starting locations $z$ away from the $\mathrm{N}$ splitting surfaces. Thus, for $z \neq z_{i}(i=1,2, \cdots, N)$, it is shown in Eq. (H.2.15):

$$
\begin{aligned}
& -\mu \frac{\partial}{\partial z} F_{2}(z, \mu, w)+\Sigma_{T} F_{2}(z, \mu, w)=\int d \mu^{-} \Sigma_{s}^{*} \cdot E\left(z, \mu \rightarrow \mu^{-\infty}\right) F_{2}\left(z, \mu^{-1}, w\right) \\
& \quad+H(\mu) \delta(z-D) \cdot \frac{\mu}{\Sigma_{T}} \cdot w^{2},
\end{aligned}
$$

where

$$
\begin{aligned}
& F_{2}(z, \mu, w) \equiv 1 / \Sigma_{T} \cdot M_{2}(z, \mu, w) \\
& \Sigma_{S}^{*}=w_{S}^{2} \Sigma_{T} .
\end{aligned}
$$

Again, the form of Eq. (5.1.9) is identical to that of Eq. (4.1.2.31). The boundary conditions associated with $\mathrm{Fq} \cdot(5.1 .9)$ are

$$
\begin{aligned}
& \mathrm{F}_{2}(0, \mu<0,1)=0 \\
& \mathrm{~F}_{2}(\mathrm{D}, \mu>0,1)=\frac{1^{2}}{\Sigma_{\mathrm{T}}^{\prime}}=\frac{1}{\Sigma_{\mathrm{T}}} .
\end{aligned}
$$

However, for the case of multiple splitting surfaces, a d*scontinuity exists in the seconi. moment solution at each splitting surface. The nature of the discontinuity at each surface is identical to the condition found in Chapter IV when considering a single surface. However, the condition is derived for the general case in Appendix $H$. At each $z_{i}(t=1,2, \cdots, N):$ 
for $\mu<0$

$$
\mathrm{M}_{2}^{-}\left(z_{i}, \mu, w\right)=\frac{1}{m_{i}} M_{2}^{+}\left(z_{i}, \mu, w\right)
$$

and for $\mu>0$

$M_{2}^{+}\left(z_{i}, \mu, w\right)=m_{i} \cdot M_{2}^{-}\left(z_{i}, \mu, w\right)-\left(m_{i}-1\right) \cdot\left\{M_{1, z_{i}}\left(z_{i}, \mu, w\right)\right\}^{2}$,

where

$$
\begin{aligned}
& M_{2}^{-}\left(z_{i}, \mu, w\right)=\underset{z^{+} z_{i-}}{\lim } M_{2, i}(z, \mu, w) \\
& M_{2}^{+}\left(z_{i}, \mu, w\right)=\lim _{z \rightarrow z_{i+}} M_{2, i+1}(z, \mu, w) .
\end{aligned}
$$

The discrete ordinates transport code ONETRAN was again employed to solve the adjoint formulations of the Boltzmann transport equation, i.e., Eqs. (5.1.7) and (5.1.9). The first moment solution was determined initially and provided the information required for the discontinuities in the second moment calculation [see Eq. (5.1.11b)]. As in the case of one surface, the second moment calculation required a slight. modification in the ONETRAN program in order to allow for the discontinuity conditions at each splitting surface (see Section 4.3.3).

\subsection{Time per Particle History $\left(\tau_{p}\right)$}

As in Chapter IV, the formulations for the first moment (expected value) of time per particle history are derived directly from the r-th moment $(r=1)$ equations, $1 . e .$, the formulations for $M_{r}(P)$. Now, however, there are $N+1$ regions and $N$ internal splitting surfaces in the problem geometry. The total score accumulation probability as formulated for 
the six cases in Section 5.1 still pertains here. In fact, the entire derivation of the moment equations, up through the separation of $r$-th moment terms from non-r-th moment terms, is still applicable (see Appendix H). The divergence in the derivations comes about when consideration is first given to the nature of the score distribution functions. In particular, use is made of the fact that the particle weight has no effect on the score distributions in the time problem (recall that computer times required to process events are considered to be "tallied" in this case). Thus, there is no dependence on weight in the expected time to process a particle history $\left[\tau_{p}(R)\right]$. All weightdependence is eliminated from the formulations formally by performing the indicated integrations over weight in the general $M_{r}(P)$ equations $(r=1)$.

In addition to the definitions made in Section 4.2 , we define the following weight-independent kernels and probability distributions which will appear in the multi-surface $\tau_{p}(\underline{R})$ equations. First,

$$
\mathrm{S}_{\mathrm{m}_{i}}\left(\underline{\mathrm{R}}^{+}\right)=\mathrm{S}_{\mathrm{m}_{1}}\left(\underline{\mathrm{P}}^{+}\right)
$$

and

$$
R_{m_{i}}\left(\underline{R}^{+}\right)=R_{m_{i}}\left(\underline{p}^{+}\right) \text {, }
$$

since these distributions have no dependence on weight $w^{+}$(see definitions in Section 5.1). Also for the multiple-surface problem 


$$
\begin{aligned}
& \mathrm{D}_{0}\left(\underline{\mathrm{R}}, \underline{\mathrm{R}}^{+}\right) \mathrm{d \underline { \textrm {R } } ^ { + }} \quad=\int \mathrm{dw}{ }^{+} \mathrm{D}_{0}\left(\underline{\mathrm{P}}, \underline{\mathrm{P}}^{+}\right) \mathrm{d \textrm {R } ^ { + }} \\
& =\left\{\begin{aligned}
\mathrm{T}\left(\mathrm{z} \rightarrow \mathrm{z}^{+}, \mu\right)[1-\mathrm{H}(\mu)] \delta\left(\mu^{+}-\mu\right) \delta\left(\mathrm{z}^{+}\right) \mathrm{d} \mathrm{z}^{+} \mathrm{d} \mu^{+} \\
\\
\text {for } \mathrm{z} \leq \mathrm{z}_{1} \\
0 \text { for } z>z_{1},
\end{aligned}\right. \\
& \mathrm{D}_{\mathrm{T}}\left(\underline{\mathrm{R}}, \underline{\mathrm{R}}^{+}\right) \underline{\mathrm{dR}}^{+} \quad \equiv \int \mathrm{dw} \mathrm{w}^{+} \mathrm{\nu}_{\mathrm{T}}\left(\underline{\mathrm{P}}, \underline{\mathrm{P}}^{+}\right) \mathrm{d \textrm {R } ^ { + }}, \\
& =\left\{\begin{array}{c}
T\left(z \rightarrow z^{+}, \mu\right) H(\mu) \delta\left(\mu^{+}-\mu\right) \delta\left(z^{+}-D\right) d z^{+} d \mu^{+} \\
\text {for } z \geq z_{N} \\
0 \quad \text { for } \quad z<z_{N}
\end{array}\right. \\
& \beta_{0 m_{i}}\left(\underline{R}^{+}, \underline{R}^{\prime \prime}\right) d \underline{R}^{\prime \prime} \equiv \int d w^{\prime} \beta_{0 m_{i}}\left(\underline{P}^{+}, \underline{p}^{\prime \prime}\right) d \underline{R}^{\prime}, \\
& =\xi_{\mathrm{m}_{i}} \delta\left(z^{\prime \prime}-z^{+}\right) \delta\left(\mu^{\prime \prime}-\mu^{+}\right) \mathrm{d} z^{\prime} \mathrm{d} \mu^{\prime \prime} \text {. }
\end{aligned}
$$

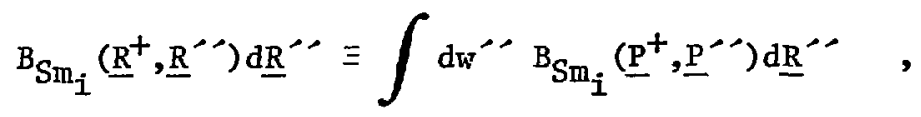

$$
\begin{aligned}
& =\delta\left(z^{\prime}-z^{+}\right) \delta\left(\mu^{\prime}-\mu^{+}\right) \mathrm{d} z^{\prime} d \mu^{\prime} \text {. }
\end{aligned}
$$

The definitions for $T\left(\underline{R}, \underline{R}^{+}\right), \sigma\left(\underline{R}^{+}, \underline{R}^{\prime}\right)$, and $E\left(\underline{R}^{-}, \underline{R}^{-}\right)$are as given in Section 4.2. Again (as in Section 4.2), the actual computer time required to process a particular event is considered to constitute the score at each event. The score distribution functions are thus expressed: 


$$
\begin{aligned}
& \mathrm{p}_{\mathrm{S}}(\underline{R}, \mathbf{s}) \mathrm{ds}=\mathrm{P}_{\mathrm{S}}(\underline{P}, \mathbf{s}) \mathrm{d} \mathbf{s}=\delta\left(s-\tau_{\mathrm{c}}\right) \mathrm{d} \mathbf{s} \\
& \mathrm{P}_{E, 0}(\underline{R}, s) d s=\mathrm{p}_{E, 0}(\underline{P}, s) \mathrm{ds}=\delta\left(s-\tau_{0}\right) \mathrm{ds} \\
& \mathrm{p}_{E, D}(\underline{R}, s) d s=p_{E, D}(\underline{P}, s) d s=\delta\left(s-\tau_{T}\right) d s \\
& \mathrm{P}_{\mathrm{Sm}_{i}}(\underline{R}, s) \mathrm{ds}=\mathrm{P}_{\mathrm{Sm}}(\underline{\mathrm{P}}, \mathrm{s}) \mathrm{d} s=\delta\left(s-\tau_{s, i}\right) \mathrm{d} s \\
& \mathrm{P}_{\mathrm{R}_{i}}(\underline{R}, s) \mathrm{d} s=\mathrm{P}_{\mathrm{R}_{i}}(\underline{P}, s) \mathrm{d} s=\delta\left(s-\tau_{\mathrm{R}, \mathrm{i}}\right) \mathrm{ds} \text {, }
\end{aligned}
$$

where $\tau_{c}, \tau_{0}, \tau_{T}$ are the actual computer times required to process a scattering collision, a front-surface escape, and tally-surface escape (respectively). Also, $\tau_{s, i}$ and $\tau_{R, i}$ represent the times required to process an $m_{i}: 1$ splitting event and Russian roulette event (respectively) at the i-th splitting surface.

Given the kernels and distributions above, the integral equations for the expected time per particle history $\left[\tau_{p}(z, \mu)\right]$ may be developed for each of the six cases considered in section 5.1.

As mentioned previously, the starting point in this development involves the $\mathrm{r}$-th moment equations as derived from the score accumulation probabilities for the various cases discussed in Section 5.1 [see Eq. (H.0.1)]. Specifically, we consider the $M_{r}(\underline{P})$ equations $(r=1)$ at a point in their development where the terms $M_{Y}(\underline{P})$ have been collected, and non-r-th moment terms appear in a source-like term $Q_{r}(\underline{F})$. The equations in question resemble Eqs. (B.1.11,-13); however now six formulations could be written, one for each of the cases presented in Section 5.1 . 
For example, the appropriate equation for the first moment $(x=1)$ is written here for particle starting positions $z$ in the range

$$
\begin{aligned}
& z_{i-1}<z<z_{i} \text { (region i): } \\
& M_{1, i}(\underline{P})=\int d \underline{P}^{+} T\left(\underline{P}, \underline{P}^{\prime}\right) \int d \underline{P}^{\prime} \sigma\left(\underline{P}^{+}, \underline{P}^{\prime}\right) \int d \underline{P}^{\prime} E\left(\underline{P}^{\prime}, \underline{P}^{\prime \prime}\right) \\
& z_{i-1}<z^{+}<z_{i} \\
& \times \mathrm{M}_{1, i}\left(\underline{\mathrm{P}}^{-\prime}\right)+\int \mathrm{dP}^{+} \mathrm{T}\left(\underline{\mathrm{P}}, \underline{\mathrm{P}}^{+}\right) \mathrm{R}_{\mathrm{m}_{i-1}}\left(\underline{\mathrm{P}}^{+}\right) \int \underline{\mathrm{d}}^{\prime-} \mathrm{B}_{0 \mathrm{~m}_{i-1}}\left(\xi_{\mathrm{m}_{i-1}} ; \underline{\mathrm{P}}^{+}, \mathrm{P}^{\prime \prime}\right) \\
& \times M_{1, z_{i-1}}\left(\underline{F}^{\prime \prime}\right)+\int d \underline{P}^{+} T\left(\underline{P}, \underline{P}^{+}\right) S_{m_{i}}\left(\underline{P}^{+}\right) \int \underline{\mathrm{P}}^{-\prime} \mathrm{B}_{\mathrm{Sm}_{i}}\left(\underline{\mathrm{P}}^{+}, \underline{\mathrm{P}}^{\prime-}\right) \cdot \mathrm{m}_{i} \\
& \times \mathrm{M}_{1, z}\left(\underline{\mathrm{P}}^{\prime \prime}\right)+\mathrm{Q}_{1, i}(\underline{\mathrm{P}}) \text {, }
\end{aligned}
$$

where

$$
\begin{aligned}
& \mathrm{Q}_{1, i}(\underline{\mathrm{P}})=\int d \underline{\mathrm{P}}^{+} \mathrm{T}\left(\underline{\mathrm{P}}, \underline{\mathrm{P}}^{+}\right) \int \mathrm{d \underline {P } ^ { - }} \sigma\left(\underline{\mathrm{P}}^{+}, \underline{\mathrm{P}}^{\prime}\right) \int \mathrm{d} s \mathrm{p}_{\mathrm{S}}\left(\underline{\mathrm{P}}^{-}, \mathrm{s}\right) \mathrm{s} \\
& z_{i-1}<z^{+}<z_{i} \\
& +\int \underline{\mathrm{P}}^{+} \mathrm{T}\left(\underline{\mathrm{P}}, \underline{\mathrm{P}}^{+}\right) \mathrm{R}_{\mathrm{m}_{\mathrm{i}-1}}\left(\underline{\mathrm{P}}^{+}\right) \int \mathrm{d} s \mathrm{p}_{\mathrm{R}_{\mathrm{i}-1}}\left(\underline{\mathrm{P}}^{+}, \mathrm{s}\right) \mathrm{s} \\
& +\int \underline{\mathrm{p}}^{+} \mathrm{T}\left(\underline{\mathrm{P}}, \underline{\mathrm{P}}^{+}\right) \mathrm{s}_{\mathrm{m}_{\mathrm{i}}}\left(\underline{\mathrm{P}}^{+}\right) \int \mathrm{d} s \mathrm{p}_{\mathrm{Sm}_{i}}\left(\underline{\mathrm{P}}^{+}, \mathrm{s}\right) \mathrm{s} \text {. }
\end{aligned}
$$

The integral over $\mathrm{dP}^{\prime \prime}$ in the second term of Eq. (5.2.2a) may be simplified. Recall from the definition of $\mathrm{B}_{\left(\mathrm{m}_{i}\right.}$ (Section 5.1),

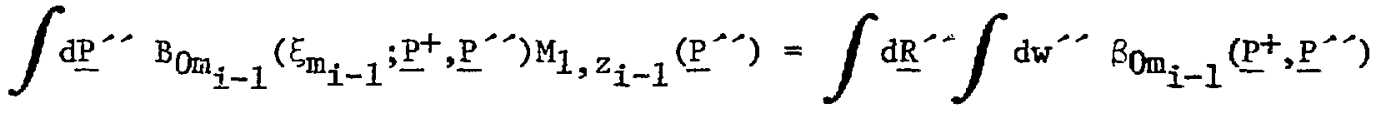

$$
\begin{aligned}
& \left.\times M_{1, z_{i-1}}{\underline{\left(P^{\prime}\right.}}^{\prime}\right)+\int \mathrm{dR}^{\prime} \int \mathrm{d} w^{\prime} \delta\left(z^{\prime}-z^{+}\right) \delta\left(\mu^{\prime}-\mu^{+}\right) \\
& \times\left(1^{\circ}-\xi_{m_{1-1}}\right) \delta\left(w^{\prime \prime}\right) M_{1, z_{i-1}}\left(\underline{R}^{\prime+}, w^{\prime-}\right) \text {. }
\end{aligned}
$$

However, by the definition in Eq. (4.1.1.6) 


$$
\int \delta\left(w^{\prime}\right) M_{1, z_{i-1}}\left(\underline{R}^{-}, w^{-}\right) d w^{-}=M_{1, z_{i-1}}\left(R^{-}, 0\right) \equiv 0 .
$$

Thus, Eq. (5.2.3) becomes

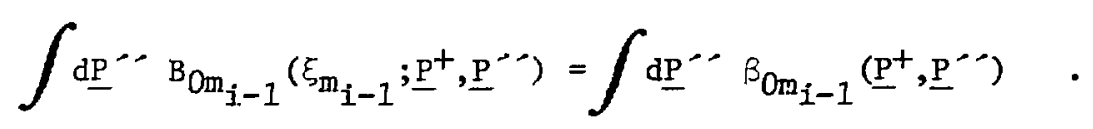

Since we are now considering the expected value of the time per particle history, for notational =larity the symbol for the function is changed from $M_{1, i}$ to ${ }^{T} p, i$. Also, as noted before, the weight of a particle now has no bearing at all on the scores and, hence, on the first moment of the time per particle history. The weight-independence is formally stated:

$$
M_{1, i}(\underline{P}) \equiv \tau_{p, i}(\underline{P})=\tau_{p, i}(\underline{R}) \quad .
$$

Finally, the implied integrations over weight are carried out in Eqs. (5.2.2a,b). Using Eqs. (5.2.6), (5.2.5), and tue definitions for the kernels stated earlier, Eqs. (5.2.2a,b) become

$$
\begin{aligned}
& \tau_{p, i}(\underline{R})=\int d \underline{R}^{+} T\left(\underline{R}, \underline{R}^{+}\right) \int d \underline{R}^{\prime} \sigma\left(\underline{R}^{+}, \underline{R}^{\prime}\right) \int \mathrm{dR}^{\prime \prime} E\left(\underline{R}^{\prime}, \underline{R}^{\prime \prime}\right) \tau_{p, i}\left(\underline{R}^{\prime \prime}\right) \\
& z_{i-1}<z^{+}<z_{i}
\end{aligned}
$$

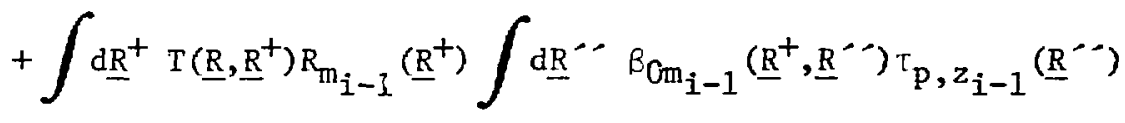

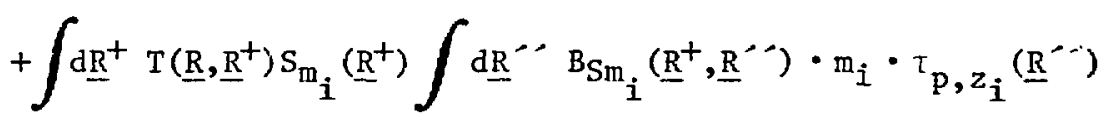

$$
\begin{aligned}
& +Q_{p, i}(\underline{R}) \quad,
\end{aligned}
$$


where

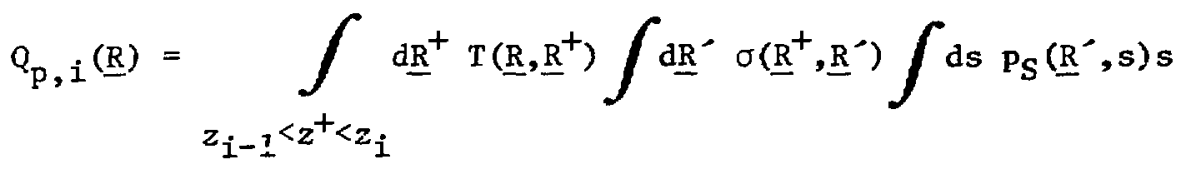

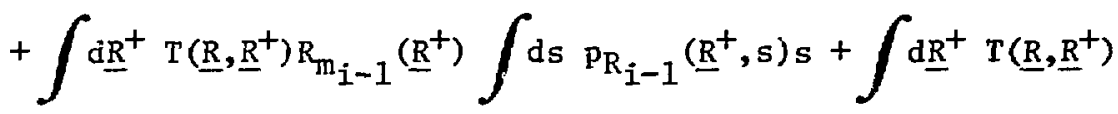

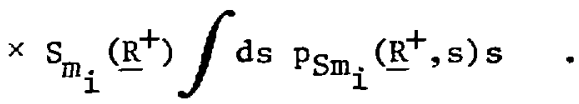

The $\tau_{p}$ equations for the remaining five cases may be similarly derived. For particles with starting locations in region $1\left(0 \leq z<z_{1}\right)$ :

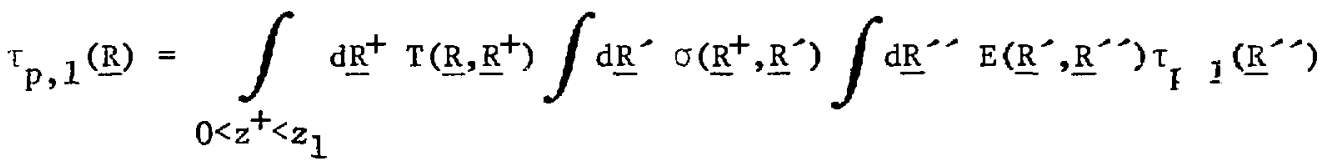

$$
\begin{aligned}
& +\int \mathrm{d}^{+} \mathrm{T}\left(\underline{\mathrm{R}}, \underline{\mathrm{R}}^{+}\right) \mathrm{S}_{\mathrm{m}_{1}}\left(\underline{\mathrm{R}}^{+}\right) \int \underline{\mathrm{R}}^{\cdots} \mathrm{B}_{\mathrm{Sm}_{1}}\left(\underline{\mathrm{R}}^{+}, \underline{\mathrm{R}}^{\prime-}\right) \cdot \mathrm{m}_{1} \cdot \tau_{\mathrm{p}, \mathrm{z}_{1}}\left(\underline{\mathrm{R}}^{\prime}\right) \\
& +Q_{p, 1}(\underline{R}) \quad,
\end{aligned}
$$

where

$$
\begin{aligned}
\mathrm{Q}_{\mathrm{p}, 1} \underline{(\mathrm{R})} & =\int \underline{\mathrm{R}}^{+} \mathrm{D}_{0}\left(\underline{\mathrm{R}}, \underline{\mathrm{R}}^{+}\right) \int \mathrm{d} s \mathrm{p}_{\mathrm{E}, 0}\left(\underline{\mathrm{R}}^{+}, \mathrm{s}\right) \mathrm{s}+\int_{0<\mathrm{z}^{+}<\mathrm{z}_{1}} \mathrm{d \underline {R } ^ { + }} \mathrm{T}\left(\underline{\mathrm{R}}, \underline{\mathrm{R}}^{+}\right) \\
& \left.\times \int \underline{\mathrm{d}}^{-} \sigma\left(\underline{\mathrm{R}}^{+}, \underline{\mathrm{R}}^{\prime}\right) \int \mathrm{ds} \mathrm{p}_{\mathrm{S}}\left(\underline{\mathrm{R}}^{-}, \mathrm{s}\right) \mathrm{s}+\int \underline{\mathrm{dR}}^{+} \mathrm{T}\left(\underline{\mathrm{R}}, \underline{\mathrm{R}}^{+}\right) \mathrm{s}_{\mathrm{m}_{1}} \underline{(\mathrm{R}}^{+}\right) \\
& \left.\times \int \mathrm{ds} \cdot{ }^{\mathrm{P}} \mathrm{Sm}_{1} \underline{(R)}^{+}, \mathrm{s}\right) \mathrm{s} .
\end{aligned}
$$

154 
For particles proceeding from surface $1\left(z=z_{1}\right)$ :

$$
\begin{aligned}
& \tau_{p, z_{1}}(\underline{R})=\int_{0<z^{+}<z_{1}} \underline{d R}^{+} T\left(\underline{R}, \underline{R}^{+}\right) \int \underline{R}^{-} \sigma\left(\underline{R}^{+}, \underline{R}^{\prime}\right) \int \underline{R}^{-1} E\left(\underline{R}^{-}, \underline{R}^{\prime \prime}\right) \tau_{p, 1}\left(\underline{R}^{\prime \prime}\right) \\
& \left.+\int_{z_{1}<z^{+}<z_{2}} d \underline{R}^{+} T\left(\underline{R}, \underline{R}^{+}\right) \int d \underline{R}^{\prime} \sigma\left(\underline{R}^{+}, \underline{R}^{\prime}\right) \int \underline{R}^{\prime \prime} E\left(\underline{R}^{\prime}, \underline{R}^{\prime \prime}\right) \tau, p, \underline{R}^{\prime \prime}\right) \\
& +\int \mathrm{d} \underline{\mathrm{R}}^{+} \mathrm{T}\left(\underline{\mathrm{R}}, \underline{\mathrm{R}}^{+}\right) \mathrm{S}_{\mathrm{m}_{2}}\left(\underline{\mathrm{R}}^{+}\right) \int \mathrm{d}^{\prime}{ }^{\prime} \mathrm{B}_{\mathrm{Sm}_{2}}\left(\underline{\mathrm{R}}^{+}, \underline{\mathrm{R}}^{\prime \prime}\right) \cdot \mathrm{m}_{2} \cdot \tau_{\mathrm{p}, \mathrm{z}_{2}}\left(\underline{\mathrm{R}}^{\prime \prime}\right) \\
& +Q_{p, z_{1}}(\underline{R}),
\end{aligned}
$$

where

$$
\begin{aligned}
& \mathrm{Q}_{\mathrm{p}, \mathrm{z}_{1}}=\int \mathrm{d \underline {R }}^{+} \mathrm{D}_{0}\left(\underline{\mathrm{R}}, \underline{\underline{R}}^{+}\right) \int \mathrm{ds} \mathrm{P}_{\mathrm{E}, 0}\left(\underline{\mathrm{R}}^{+}, \mathrm{s}\right) \mathrm{s}+\int \underline{\mathrm{R}}^{+} \mathrm{T}\left(\underline{\mathrm{R}}, \underline{\mathrm{R}}^{+}\right) \mathrm{s}_{\mathrm{m}_{2}}\left(\underline{\mathrm{R}}^{+}\right) \int \mathrm{ds} \\
& \left.\times \operatorname{P}_{\mathrm{Sm}_{2}} \underline{(R}^{+}, \mathrm{s}\right) \mathrm{s}+\int_{0<\mathrm{z}^{+}<\mathrm{z}_{1}} \underline{\mathrm{d}}^{+} \mathrm{T}\left(\underline{\mathrm{R}}, \underline{\mathrm{R}}^{+}\right) \int \underline{\mathrm{dR}}^{-} \sigma\left(\underline{\mathrm{R}}^{+}, \underline{\mathrm{R}}^{\prime}\right) \int \mathrm{d} s \\
& \left.\times \mathrm{P}_{\mathrm{S}} \underline{\mathrm{R}}^{-}, \mathrm{s}\right) \mathrm{s}+\int_{z_{1}<\mathrm{z}^{+}<z_{2}} \underline{\mathrm{dR}}^{+} \mathrm{T}\left(\underline{\mathrm{R}}, \underline{\mathrm{R}}^{+}\right) \int \underline{\mathrm{R}}^{-} \sigma\left(\underline{\mathrm{R}}^{+}, \underline{R}^{\prime}\right) \int \mathrm{ds} \\
& \times P_{S}\left(\underline{R}^{\prime}, s\right) s
\end{aligned}
$$


For particies proceeding from surface $i\left(z=z_{i}\right)$ :

$$
\begin{aligned}
& \tau, p, z_{i}(\underline{R})=\int d \underline{\mathrm{R}}^{+} \mathrm{T}\left(\underline{\mathrm{R}}, \underline{\mathrm{R}}^{+}\right) \int \mathrm{d \underline {R } ^ { - }} \sigma\left(\underline{\mathrm{R}}^{+}, \underline{\mathrm{R}}^{-}\right) \int \mathrm{d \underline {R } ^ { \prime }} \mathrm{E}\left(\underline{\mathrm{R}}^{\prime}, \underline{\mathrm{R}}^{-}\right) \\
& z_{i-1}<z^{+}<z_{i} \\
& \times \tau_{p, i}\left(\underline{R}^{\prime \prime}\right)+\int_{z_{i}<z^{+}<z_{i+1}} d_{R^{+}} T\left(\underline{R}, \underline{R}^{+}\right) \int d \underline{R}^{\prime} \sigma\left(\underline{R}^{+}, \underline{R}^{\prime}\right) \int \underline{R}^{\prime \prime} \\
& \left.\left.\times E\left(\underline{R}^{\prime}, \underline{R}^{-\prime}\right) \tau_{p, i+1} \underline{R}^{\prime \prime}\right)+\int d \underline{R}^{+} T\left(\underline{R}, \underline{R}^{+}\right) R_{m_{i-1}} \underline{R}^{+}\right) \int d \underline{R}^{\prime} \\
& \left.\times \beta_{0 m_{i-1}}\left(\underline{R}^{+}: \underline{R}^{\prime \prime}\right) \tau, p, z_{i-1}\left(\underline{R}^{\prime \prime}\right)+\int \underline{d R}^{+} T\left(\underline{R}, \underline{R}^{+}\right) S_{m_{i+1}} \underline{R}^{+}\right) \int \underline{d R}^{\prime-} \\
& \left.\left.\times \mathrm{B}_{\mathrm{S} \mathrm{m}_{i+1}}\left(\underline{\mathrm{R}}^{+}, \underline{\mathrm{R}}^{\prime \prime}\right) \cdot \mathrm{m}_{i+1} \cdot \tau_{\mathrm{p}, z_{i+1}} \underline{\mathrm{R}}^{\prime \prime}\right)+\mathrm{Q}_{\mathrm{p}, z_{i}} \underline{\mathrm{R}}\right),
\end{aligned}
$$

where

$$
\begin{aligned}
& \mathrm{Q}_{\mathrm{p}, z_{i}}(\underline{\mathrm{R}})=\int_{z_{i-1}<z^{+}<z_{i}} \underline{\mathrm{R}}^{+} \mathrm{T}\left(\underline{\mathrm{R}}, \underline{R}^{+}\right) \int \underline{\mathrm{d}}^{-} \sigma\left(\underline{R}^{+}, \underline{R}^{-}\right) \int \mathrm{ds} \mathrm{p}_{S}\left(\underline{\mathrm{R}}^{-}, \mathrm{s}\right) \mathrm{s} \\
& +\int_{z_{i}<z^{+}<z_{i+1}} \underline{d R}^{+} T\left(\underline{R}, \underline{R}^{+}\right) \int d \underline{R}^{\prime} \sigma\left(\underline{R}^{+}, \underline{R}^{-}\right) \int d s p_{S}\left(\underline{R}^{\prime}, s\right) s \\
& \left.+\int \underline{\mathrm{dR}}^{+} \mathrm{T}\left(\underline{\mathrm{R}}, \underline{\mathrm{R}}^{+}\right) \mathrm{R}_{\mathrm{m}_{\mathrm{i}-1}} \underline{\mathrm{R}}^{+}\right) \int \mathrm{ds} \mathrm{P}_{\mathrm{R}_{\mathrm{i}-1}}\left(\underline{\mathrm{R}}^{+}, \mathrm{s}\right) \mathrm{s}+\int \mathrm{dR^{+ }} \mathrm{T}\left(\underline{\mathrm{R}}, \underline{\mathrm{R}}^{+}\right) \\
& \times \mathrm{s}_{\mathrm{m}_{i+1}}\left(\underline{R}^{+}\right) \int \mathrm{ds} \mathrm{p}_{\mathrm{Sm}_{\mathrm{i}+1}}\left(\underline{\mathrm{R}}^{+}, \mathrm{s}\right) \mathrm{s} .
\end{aligned}
$$


For particles proceeding from surface $N\left(z=z_{N}\right)$ :

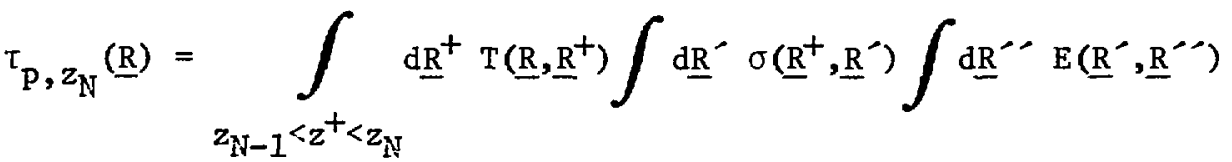

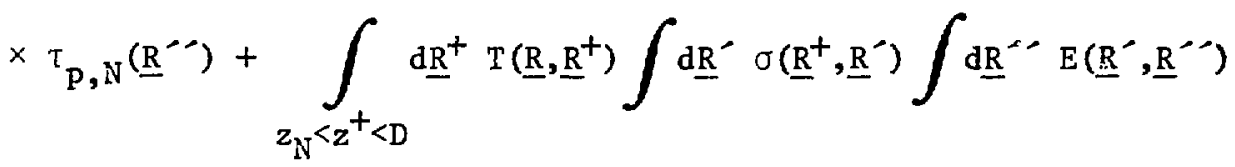

$$
\begin{aligned}
& \times \tau_{p, N+1}\left(\underline{R}^{\prime \prime}\right)+\int \mathrm{dR}^{+} \mathrm{T}\left(\underline{\mathrm{R}}, \underline{\mathrm{R}}^{+}\right) \mathrm{R}_{\mathrm{m}_{\mathrm{N}-1}}\left(\underline{\mathrm{R}}^{+}\right) \int \mathrm{d}^{\prime}{ }^{\prime} \beta_{0 \mathrm{~m}_{\mathrm{N}-1}}\left(\underline{\mathrm{R}}^{+}, \underline{\mathrm{R}}^{\prime \prime}\right) \\
& \times \tau_{p, z_{N-1}}\left(\underline{R}^{\prime \prime}\right)+Q_{p, z_{N}}(\underline{R}),
\end{aligned}
$$

where

$$
\begin{aligned}
& \mathrm{Q}_{\mathrm{p}, \mathrm{z}_{\mathrm{N}}}(\underline{\mathrm{R}})=\int_{\mathrm{z}_{\mathrm{N}-1}<\mathrm{z}^{+}<\mathrm{z}_{\mathrm{N}}} \underline{\mathrm{dR}}^{+} \mathrm{T}\left(\underline{\mathrm{R}}, \underline{\mathrm{K}}^{+}\right) \int \underline{\mathrm{R}}^{\prime} \sigma\left(\underline{\mathrm{R}}^{+}, \underline{\mathrm{R}}^{\prime}\right) \int \mathrm{ds} \mathrm{p}_{\mathrm{S}}\left(\underline{\mathrm{R}}^{\prime}, \mathrm{s}\right) \mathrm{s} \\
& +\int_{z_{N}<z^{+}<D} \underline{d R}^{+} T\left(\underline{R}, \underline{R}^{+}\right) \int d \underline{R}^{-} \sigma\left(\underline{R}^{+}, \underline{R}^{\prime}\right) \int d s p_{S}\left(\underline{R}^{-}, s\right) s \\
& \left.+\int \underline{\mathrm{R}}^{+} \mathrm{T}\left(\underline{\mathrm{R}}, \underline{\mathrm{R}}^{+}\right) \mathrm{R}_{\mathrm{m}-1}{\underline{\left(\mathrm{R}^{+}\right.}}\right) \int \mathrm{ds} \mathrm{p}_{\mathrm{R}_{\mathrm{N}-1}}\left(\underline{\mathrm{R}}^{+}, \mathrm{s}\right) \mathrm{s}+\int \underline{\mathrm{d}}^{+} \\
& \times D_{T}\left(\underline{R}, \underline{R}^{+}\right) \int d s p_{E, D}\left(\underline{R}^{+}, s\right) s .
\end{aligned}
$$

157 
Finally, for particles beginning their flight in region $\mathrm{N}+1$ $\left(z_{N}<z \leq D\right):$

$$
\begin{aligned}
& \tau_{p, N+1}(\underline{R})=\int_{z_{N^{<}} z^{+}<D} \underline{\mathrm{R}}^{+} \mathrm{T}\left(\underline{\mathrm{R}}, \underline{\mathrm{R}}^{+}\right) \int \underline{\mathrm{d}}^{\prime} \sigma\left(\underline{\mathrm{R}}^{+}, \underline{\mathrm{R}}^{\prime}\right) \int \underline{\mathrm{d}}^{-} \mathrm{E}\left(\underline{\mathrm{R}}^{-}, \underline{\mathrm{R}}^{\prime \prime}\right) \\
& \times \tau_{p, N+1}\left(\underline{R}^{\prime \prime}\right)+\int d \underline{R}^{+} T\left(\underline{R}, \underline{R}^{+}\right) R_{m_{N}}\left(\underline{R}^{+}\right) \int d \underline{R}^{-1} B_{O m_{N}}\left(\underline{R}^{+}, \underline{R}^{-\rho}\right) \\
& \times \tau_{\mathrm{p}, \mathrm{z}_{\mathrm{N}}}\left(\underline{\mathrm{R}}^{\cdots}\right)+\mathrm{Q}_{\mathrm{p}, \mathrm{N}+1}(\underline{\mathrm{R}}),
\end{aligned}
$$

where

$$
\begin{aligned}
\mathrm{Q}_{\mathrm{p}, \mathrm{N}+1}(\underline{\mathrm{R}}) & =\int_{z_{\mathrm{N}}<\mathrm{z}^{+}<\mathrm{D}} \mathrm{d}^{+} \mathrm{T}\left(\underline{\mathrm{R}}, \underline{\mathrm{R}}^{+}\right) \int \underline{\mathrm{R}}^{-} \sigma\left(\underline{\mathrm{R}}^{+}, \underline{\mathrm{R}}^{-}\right) \int \mathrm{ds} \mathrm{P}_{\mathrm{S}}\left(\underline{\mathrm{R}}^{-}, \mathrm{s}\right) \mathrm{s} \\
& \left.+\int \underline{\mathrm{dR}}^{+} \mathrm{T}\left(\underline{\mathrm{R}}, \underline{\mathrm{R}}^{+}\right) \mathrm{R}_{\mathrm{N}}\left(\underline{\mathrm{R}}^{+}\right) \int \mathrm{ds} \mathrm{p}_{\mathrm{R}_{\mathrm{N}}} \underline{\mathrm{R}}^{+}, \mathrm{s}\right) \mathrm{s}+\int \mathrm{dR}^{+} \mathrm{D}_{\mathrm{T}}\left(\underline{\mathrm{R}}, \underline{\mathrm{R}}^{+}\right) \\
& \left.\times \int \mathrm{d} s \mathrm{p}_{\mathrm{E}, \mathrm{D}} \underline{\mathrm{R}}^{+}, \mathrm{s}\right) \mathrm{s}
\end{aligned}
$$

Starting with Eqs. (5.2.7) through (5.2.12), a single integrodifferential equation may be developed which is applicable at all z except $z_{i}(i=1,2, \cdots, N)$. The development is outlined in Appendix I and follows very closely the derivation presented in Section 4.2 for the singie splitting surface case. Thus, Eq. (I.15) is rewritten here for all $z \neq z_{I}(i=1,2, \cdots, N)$ : 


$$
\begin{aligned}
-\mu \frac{\partial}{\partial z} F_{T}(z, \mu)+\Sigma_{T} F_{T}(z, \mu) & =\int d \mu^{\prime} \Sigma_{T} \cdot E\left(z, \mu \rightarrow \mu^{\prime \prime}\right) F_{T}\left(z, \mu^{\prime \prime}\right)+\tau_{c} \\
& +[1-H(\mu)] \delta(z-0) \frac{|\mu|}{\Sigma_{T}} \cdot \tau_{0}+H(\mu) \delta(z-D) \frac{\mu}{\Sigma_{T}} \cdot \tau_{T},(5.2 .13)
\end{aligned}
$$

where

$$
F_{T}(z, \mu) \equiv 1 / \Sigma_{T} \cdot \tau_{p}(z, \mu)
$$

Equation (5.2.13) is an adjoint formulation of the one-speed Boltzmann transport equation; in fact, it is identical to Eq. (4.2.2.12). The solution (calculated using the ONETRAN $S_{n}$ code) is subject to an isotropic distributed source $\left(\tau_{c}\right)$. The boundary sources at $z=0$ and $z=D$ implied by the delta functions in Eq. (5.2.13) are treated by Imposing non-zero adjoint boundary conditions:

$$
F_{T}(0, \mu<0)=\tau_{0} / \Sigma_{T} \text { at } \quad z=0
$$

and

$$
\mathrm{F}_{\mathrm{T}}(\mathrm{D}, \mu>0)=\tau_{\mathrm{T}} / \Sigma_{\mathrm{T}} \quad \text { at } \quad \mathrm{z}=\mathrm{D}
$$

Finally, at each splitting surface, a discontinuity condition exists for both positive and negative values of $\mu$. The discontinuity at each $z_{i}(1=1,2, \cdots, N)$ is written here

for $\mu<0$ :

$$
\tau_{p}^{+}\left(z_{i}, \mu\right)=\frac{1}{m_{i}} \cdot \tau_{p}^{-}\left(z_{i}, \mu\right)+\tau_{R, i},
$$

and for $\mu>0$ :

$$
\tau_{p}^{-}\left(z_{i}, i\right)=m_{i} \cdot \tau_{p}^{+}\left(z_{i}, \mu\right)+\tau_{s, i},
$$


where

$$
\begin{aligned}
& \tau_{p}{ }^{-}\left(z_{i}, \mu\right) \equiv \lim _{z \rightarrow z_{i-}} \tau_{p, i}(z, \mu) \\
& \tau_{p}^{+}\left(z_{i}, \mu\right) \equiv \lim _{z \rightarrow z_{i+}} \tau_{p, i+1}(z, \mu) .
\end{aligned}
$$

\subsection{Numerical $\left(\mathrm{S}_{\mathrm{n}}\right)$ Study for the Case Involving Multiple Surfaces}

Numerical results relating to several splitting surfaces in the problem geometry (semi-infinite plane) are presented here. Results for the optimal placement of two surfaces are discussed first followed by a cost comparison between optimal location of the splitting surfaces and placement based on some of the more popular rules of thumb. Finally, $S_{n}$ predictions of sample variance are compared to stochastic results from a standard Monte Carlo production code, MCNP.

\subsubsection{Overview of the Study. Due to the iterative nature of the} optimization scheme (to be discussed in Section 5.3.3), the scope of the numerical multiple-surface study was restricted. In particular, the transport properties of the problem were fixed, i.e., the slab thickness $D$ was restricted to $D=5.0$ mean free paths and the scattering ratio was fixed at $\mathrm{w}_{\mathrm{S}} \equiv \Sigma_{\mathrm{S}} / \Sigma_{\mathrm{T}}=0.10$. Moreover, only two splitting surfaces were considered and splitting at each was in a ratio $2: 1$. The purpose of the numerical optimization, then, was to determine the optimal location of the two splitting surfaces vis $\underline{z^{\prime}}$ vis the cost function $\left(\sigma_{s}{ }^{2} \tau_{p}\right)$

The particle source in the transport model has been assumed to be normally incident at $z=0$ (as presented in Chapter III). However, the 
deterministic results of the adjoint ONETRAN calculations for the moments of the transmission score and time per particle history also include values for all of the discrete angles at $z=0$, i.e., not just at $\mu=1$. Therefore, once the $S_{n}$ solution is obtained for the plane normal source, it is also possible to integrate the angular values at $z=0$ over any arbitrary source distribution to obtain the moments of the score (and $\tau_{p}$ ) given the particular angular source distribution considered. This may be done without the expense of any additional computational work (except, of course, for the integration over the source distribution). The benefit here derives from the global nature of the solution tr the adjoint $S_{n}$ problem. The two-surface optimization considered here is therefore presented in two parts--first, for the case of a particle source normally incident at $z=0$ and then for an isotropic planar source in the forward direction also at $z=0$.

\subsubsection{Monte Carlo/ $\mathrm{S}_{\mathrm{n}}$ Comparisons. A Monte Car1o code called MCSN} (see Appendix J) was written in order to treat more efficiently the multiple splitting surfaces in slab geometry. The Fortran code listing is given in Appendix J. The logical flow of MCSN follows almost exactly that of MCSI (Section 4.4.2), major differences being confined to subroutine DSURF (geometry-related operations required for calculating the distance to the next surface). Required input to MCSN is also the same as for MCS1, except that the locations of the bounding surfaces fir each of the NCL geometry cells must be input (SURCEL array), along with the relative importance of each of the ce11s (IMP array). It is assumed 
that the slab is divided into NCL planar cells by the NCL-1 splitting surfaces.

As before, in order to use the $S_{n}$ technique to predict time per particle history (now assuming execution of the Monte Carlo problem using MCSN), new computer timings are required for the processing of a collision, splitting event, Russian roulette event, as well as front and tally-surface escapes. The event timings were obtained (using the MCSN coding) by the same procedure as described in Section 4.4.2. The timings now, however, were subject to code compilation using the FTN compiler and execution on the $\mathrm{CDC}-7600$. Results are presented in Table 6.

Finally, Monte Carlo transport calculations employing the code MCSN can be used to stochastically verify the predicted values for $\sigma_{s}{ }^{2}$ and $\tau_{p}$ which were calculated using appropriately modified versions of ONETRAN, i.e., modified to allow for the multiple discontinuities accompanying the multiple splitting surfaces. Some of the Monte Carlof $s_{n}$ comparisons are presented in Tables 7 and 8 . The transport problem on which these comparisons are based involves splitting by $2: 1$ at each of two splitting surfaces in a slab of thickness $D=5.0 \mathrm{mf}$. The scattering ratio $\left(w_{s}\right)$ is constant at $w_{s}=0.10$. Table 7 represents calculations in which the particle source was normally incident at $z=0$. The Monte Carlo results in Table 7 reflect calculations in which the expected slab transmission was computed to a relative error of about 1\%. Agreement between stochastic and deterministic values is quite good; sample variance and time per history are predicted to within a couple of per cent. 


\begin{tabular}{|c|c|c|}
\hline \multirow{2}{*}{ Time to Process... } & \multicolumn{2}{|c|}{ Time } \\
\hline & Hsec & Time Units \\
\hline Collision, $\tau_{c}$ & $10.88 \pm 0.03 \mu \mathrm{sec}$ & 1.00 \\
\hline Splitting $(\mathrm{m}=2), \tau_{\mathrm{s}}$ & $19.80 \pm 0.04$ & 1.82 \\
\hline Russian Roulette $(m=2), \tau_{R}$ & $13.08 \pm 0.01$ & 1.20 \\
\hline Front-Surface Escape, $\tau_{0}$ & $11.18 \pm 0.04$ & 1.03 \\
\hline Tally-Surface Escape, $\tau_{T}$ & $10.75 \pm 0.04$ & 0.99 \\
\hline Source Particle & $0.24 \pm 0.01$ & 0.02 \\
\hline History Update & $3.32 \pm 0.01$ & 0.30 \\
\hline
\end{tabular}

Table 6. MCSN Event Timing Summaries. 


\begin{tabular}{|c|c|c|c|c|}
\hline$\left(z_{i} \frac{\text { CASE }}{\text { in } m f p}\right)$ & & $\begin{array}{l}\text { Monte Carlo } \\
\text { (MCSN) }\end{array}$ & $\underset{\text { (ONETRAN) }}{s_{n}}$ & $\%$ Diff. \\
\hline $\begin{array}{l}z_{1}=2.00 \\
z_{2}=2.50\end{array}$ & $\begin{array}{l}\sigma_{\mathrm{s}}^{2} \\
\tau_{\mathrm{p}} \\
\mathrm{f}_{\mathrm{c}}\end{array}$ & $\begin{array}{l}2.027 \mathrm{E}-03 \\
3.668 \mathrm{E}-04(\mathrm{sec}) \\
7.437 \mathrm{E}-07(\mathrm{sec})\end{array}$ & $\begin{array}{l}1.989 \mathrm{E}-03 \\
3.661 \mathrm{E}-04(\mathrm{sec}) \\
7.282 \mathrm{E}-07(\mathrm{sec})\end{array}$ & $\begin{array}{l}-1.91 \\
-0.19 \\
-2.13\end{array}$ \\
\hline $\begin{array}{l}z_{1}=2.00 \\
z_{2}=3.00\end{array}$ & $\begin{array}{l}\sigma_{s}^{2} \\
\tau_{p} \\
f_{c}\end{array}$ & $\begin{array}{l}2.087 \mathrm{E}-03 \\
3.337 \mathrm{E}-04 \\
6.964 \mathrm{E}-07\end{array}$ & $\begin{array}{l}2.086 \mathrm{E}-03 \\
3.331 \mathrm{E}-04 \\
6.948 \mathrm{E}-07\end{array}$ & $\begin{array}{l}-0.05 \\
-0.18 \\
-0.23\end{array}$ \\
\hline $\begin{array}{l}z_{1}=2.00 \\
z_{2}=3.50\end{array}$ & $\begin{array}{c}\sigma_{\mathrm{s}}^{2} \\
\tau_{\mathrm{p}} \\
\mathrm{f}_{\mathrm{c}}\end{array}$ & $\begin{array}{l}2.259 E-03 \\
3.047 E-04 \\
6.885 E-07\end{array}$ & $\begin{array}{l}2.243 E-03 \\
3.038 E-04 \\
6.814 E-07\end{array}$ & $\begin{array}{l}-0.71 \\
-0.30 \\
-1.04\end{array}$ \\
\hline $\begin{array}{l}z_{1}=2.00 \\
z_{2}=4.00\end{array}$ & $\begin{array}{l}\sigma_{\mathrm{s}}^{2} \\
{ }_{\mathrm{f}}^{\mathrm{f}} \\
{ }_{\mathrm{C}}\end{array}$ & $\begin{array}{l}2.451 \mathrm{E}-03 \\
2.795 \mathrm{E}-04 \\
6.851 \mathrm{E}-07\end{array}$ & $\begin{array}{l}2.495 \mathrm{E}-03 \\
2.785 \mathrm{E}-04 \\
6.948 \mathrm{E}-07\end{array}$ & $\begin{array}{l}+1.76 \\
-0.36 \\
+1.40\end{array}$ \\
\hline $\begin{array}{l}z_{1}=2.00 \\
z_{2}=4.50\end{array}$ & $\begin{array}{l}\sigma_{s}^{2} \\
\tau_{p} \\
f_{c}\end{array}$ & $\begin{array}{l}2.866 \mathrm{E}-03 \\
2.583 \mathrm{E}-04 \\
7.403 \mathrm{E}-07\end{array}$ & $\begin{array}{l}2.900 E-03 \\
2.572 E-04 \\
7.459 E-07\end{array}$ & $\begin{array}{l}+1.17 \\
-0.43 \\
+0.75\end{array}$ \\
\hline
\end{tabular}

Table 7. Monte Carlo/s $\mathrm{n}$ Comparisons: Plane Normal Source. $\left[D=5.0 \mathrm{mfp} ; w_{s}=0.10 ; m_{1}=m_{2}=2\right.$ at surfaces $z_{1}$ and $\left.z_{2} \cdot\right]$ 


\begin{tabular}{|c|c|c|c|c|}
\hline$\left(z_{i} \frac{\text { CASE }}{\left.\text { in } m f_{p}\right)}\right.$ & & $\begin{array}{l}\text { Monte Carlo } \\
\text { (MCSN) }\end{array}$ & ${ }_{\text {(ONETRAN) }}^{s_{n}}$ & $\%$ Diff. \\
\hline $\begin{array}{l}z_{1}=1.00 \\
z_{2}=1.50\end{array}$ & $\begin{array}{l}\sigma_{s}^{2} \\
\tau_{p} \\
f_{c}\end{array}$ & $\begin{array}{l}2.662 \mathrm{E}-04 \\
3.690 \mathrm{E}-04(\mathrm{sec}) \\
9.822 \mathrm{E}-08(\mathrm{sec})\end{array}$ & $\begin{array}{l}2.644 \mathrm{E}-04 \\
3.662 \mathrm{E}-04 \quad(\mathrm{sec}) \\
9.684 \mathrm{E}-08(\mathrm{sec})\end{array}$ & $\begin{array}{l}-0.68 \\
-0.76 \\
-1.42\end{array}$ \\
\hline $\begin{array}{l}z_{1}=1.00 \\
z_{2}=2.00\end{array}$ & $\begin{array}{l}\sigma_{s}^{2} \\
\tau_{p} \\
f_{c}\end{array}$ & $\begin{array}{l}2.611 \mathrm{E}-04 \\
3.388 \mathrm{E}-04 \\
8.845 \mathrm{E}-08\end{array}$ & $\begin{array}{l}2.686 \mathrm{E}-04 \\
3.363 \mathrm{E}-04 \\
9.035 \mathrm{E}-08\end{array}$ & $\begin{array}{l}+2.79 \\
-0.74 \\
+2.10\end{array}$ \\
\hline $\begin{array}{l}z_{1}=1.00 \\
z_{2}=2.50\end{array}$ & $\begin{array}{l}\sigma_{\mathrm{s}}^{2} \\
{ }_{\mathrm{T}} \\
\hat{\mathrm{I}}_{\mathrm{c}}\end{array}$ & $\begin{array}{l}2.823 E-04 \\
3.104 E-04 \\
8.762 E-08\end{array}$ & $\begin{array}{l}2.759 \mathrm{E}-04 \\
3.094 \mathrm{E}-04 \\
8.535 \mathrm{E}-08\end{array}$ & $\begin{array}{l}-2.32 \\
-0.32 \\
-2.66\end{array}$ \\
\hline $\begin{array}{l}z_{1}=1.00 \\
z_{2}=3.00\end{array}$ & $\begin{array}{l}\sigma_{s}^{2} \\
\tau_{p} \\
f_{c}\end{array}$ & $\begin{array}{l}2.859 \mathrm{E}-04 \\
2.875 \mathrm{E}-04 \\
8.220 \mathrm{E}-08\end{array}$ & $\begin{array}{l}2.881 E-04 \\
2.857 E-04 \\
8.231 E-08\end{array}$ & $\begin{array}{l}+0.76 \\
-0.63 \\
+0.13\end{array}$ \\
\hline $\begin{array}{l}z_{1}=1.00 \\
z_{2}=3.50\end{array}$ & $\begin{array}{l}\sigma_{s}^{2} \\
{ }_{p} \\
{ }^{f_{c}}\end{array}$ & $\begin{array}{l}3.051 \mathrm{E}-04 \\
2.660 \mathrm{E}-04 \\
8.116 \mathrm{E}-08\end{array}$ & $\begin{array}{l}3.093 \mathrm{E}-04 \\
2.650 \mathrm{E}-04 \\
8.195 \mathrm{E}-08\end{array}$ & $\begin{array}{l}+1.36 \\
-0.38 \\
+0.96\end{array}$ \\
\hline $\begin{array}{l}z_{1}=1.00 \\
z_{2}=4.50\end{array}$ & $\begin{array}{l}\sigma_{s}^{2} \\
{ }_{s}^{\tau} p \\
f_{c}\end{array}$ & $\begin{array}{l}3.937 \mathrm{E}-04 \\
2.334 \mathrm{E}-04 \\
9.189 \mathrm{E}-08\end{array}$ & $\begin{array}{l}4.072 \mathrm{E}-04 \\
2.323 \mathrm{E}-04 \\
9.460 \mathrm{E}-08\end{array}$ & $\begin{array}{l}+3.31 \\
-0.47 \\
+2.86\end{array}$ \\
\hline
\end{tabular}

Table 8. Monte Carlo/s $\mathrm{n}_{\mathrm{n}}$ Comparisons: Isotropic Source.

$$
\left[D=5.0 \mathrm{mf}_{\mathrm{F}} ; \mathrm{w}_{\mathrm{s}}=0.10 ; \mathrm{mil}_{1}=\mathrm{m}_{2}=2 \text { at surfaces } \mathrm{z}_{1} \text {, and } \mathrm{z}_{2} \cdot\right]
$$


The results displayed in Table 8 correspond to an isotropic source at the $z=0$ plane. The Monte Carlo calculations on which Table 8 was based involve a relative error in the computed slab transmission of about $3 \%$.

\subsubsection{Optimization of Splitting Surface Locations. As mentioned} previously, the iterative nature of the optimization scheme for determiling the most cost-effective splitting parameters when employing multiple splitting surfaces precludes the extensive numerical study that was done for the single splitting surface. Thus, the optimization problem considered here was greatly restricted. Splitting in a ratio 2:1 was considered to take place at two splitting surfaces located in a slab 5.0 mean free paths in thickness. The scattering ratio was fixed at $\Sigma_{S} / \Sigma_{T}=0.10$. The goal was to find the optimal location of the two splitting surfaces within the slab. The numerical study was done for two different source conditions: first, a plane nornal source $[\mu($ source $)=1]$ was considered at $z=0$; then, an isotropic planar source (also at $z=0$ ) was investigated.

The iterative scheme for finding the optimal placement of splitting surfaces consisted of the following steps:

1) The location of the first splitting surface was fixed $\left(z=z_{1}\right)$.

2) Using $S_{n}$ calculations to predict ${ }^{\prime}{ }_{s}^{2}$ and $\tau_{p}$, a cost survey was exacuted, keeping the location of the first splitting surface,fixed while varying the location of the second $\left[\sigma_{s}{ }^{2} \tau_{p}\right.$ vs. $\bar{z}_{2}$, where $\bar{z}_{2}$ is the normalized second surface location: $\left.\bar{z}_{2} \equiv\left(z_{2}-z_{1} / D-z_{1}\right)\right]$. 
3) Based on step (2) above, a $\bar{z}_{2}$ was found at which $\sigma_{s}{ }^{2} \tau_{p}$ was minimized.

4) The location of the first splitting surface $\left(z_{1}\right)$ was shifted to a new position.

5) Steps (2) through (4) were repeated until a $\bar{z}_{2 \text { (opt) }}$ was found [for a certain $z_{1 \text { (opt) }}$ ], which resulted in the overall minimum associated cost, $\left(\sigma_{\mathrm{S}}{ }^{2} \tau_{\mathrm{p}}\right)_{\mathrm{MIN}}$.

5.3.3.a Plane Normal Source. The first source condition studied involvfd particles impinging normally $[\mu$ (source) $=1]$ on the front surface of the slab $(z=0)$.

Figures 33, 34, and 35 illustrate three of the many steps involved in the optimization procedure. In Fig. 33, with the first splitting surface iocated at $z_{1}=1.00 \mathrm{mfp}$, the cost function was minimized with $\bar{z}_{2}=0.575\left(z_{2}=3.30 \mathrm{mfp}\right)$. The cost function increased monotonically with $\bar{z}_{2}$ for $z_{1}=4.00 \mathrm{mfp}$ (see Fig. 35). However, the optimal splitting conditions (Fig. 34) were achieved with $\mathrm{z}_{1(\mathrm{opt})}=2.50 \mathrm{mfp}$ and $\overline{\mathrm{z}}_{2(\mathrm{opt})}=$ $0.475\left[z_{2(\mathrm{op} i)}=3.68 \mathrm{mfp}\right]$, resulting in $\left(\sigma_{\mathrm{s}}{ }^{2} \tau_{\mathrm{p}}\right)_{\mathrm{MIN}}=6.735 \mathrm{E}-07 \mathrm{sec}$. For reference, the non-splitting cost was $9.093 \mathrm{E}-07 \mathrm{sec}$.

5.3.3.b Isotropic Flanar Source. We define the angular source distribution at $z$ as

$$
\begin{aligned}
& S(z, \mu, w) d \mu \equiv \text { probability of a source particle being } \\
& \text { introciuced at } z \text { in } d \mu \text { about } \mu \text { (with } \\
& \text { starting weight } w \text { ). }
\end{aligned}
$$

Then, if we consider a forward-directed isotropic source at $z=0$, 


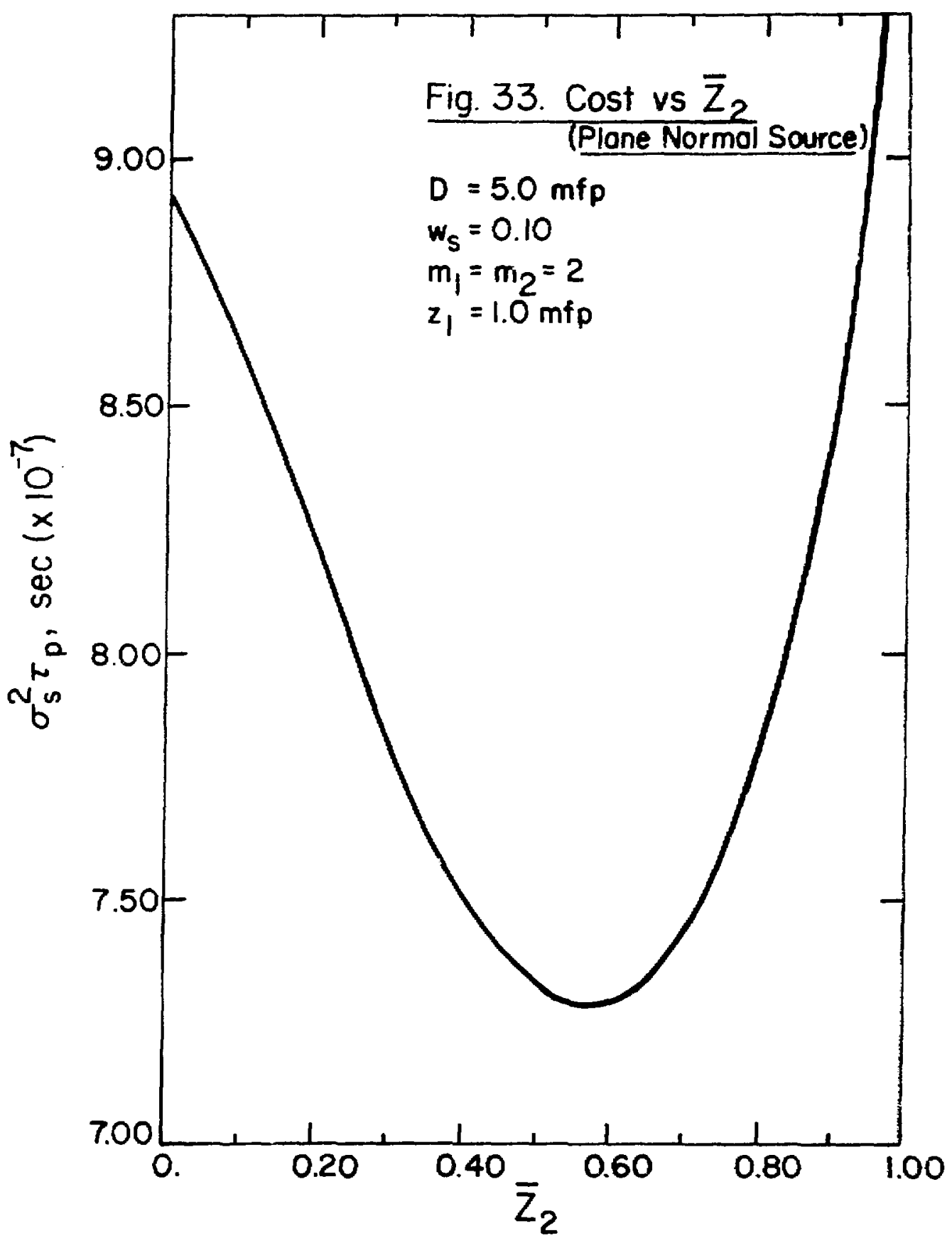




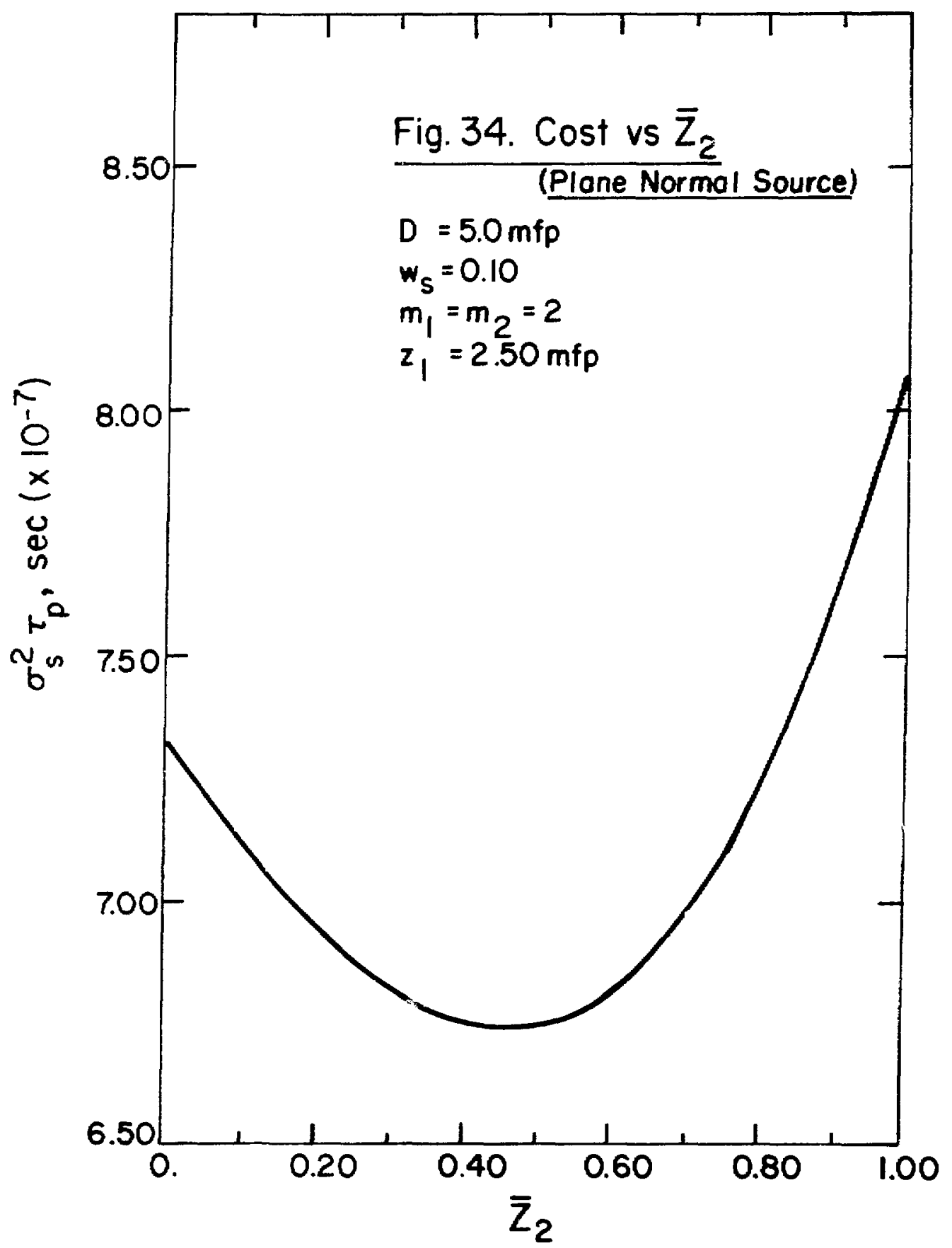

169 


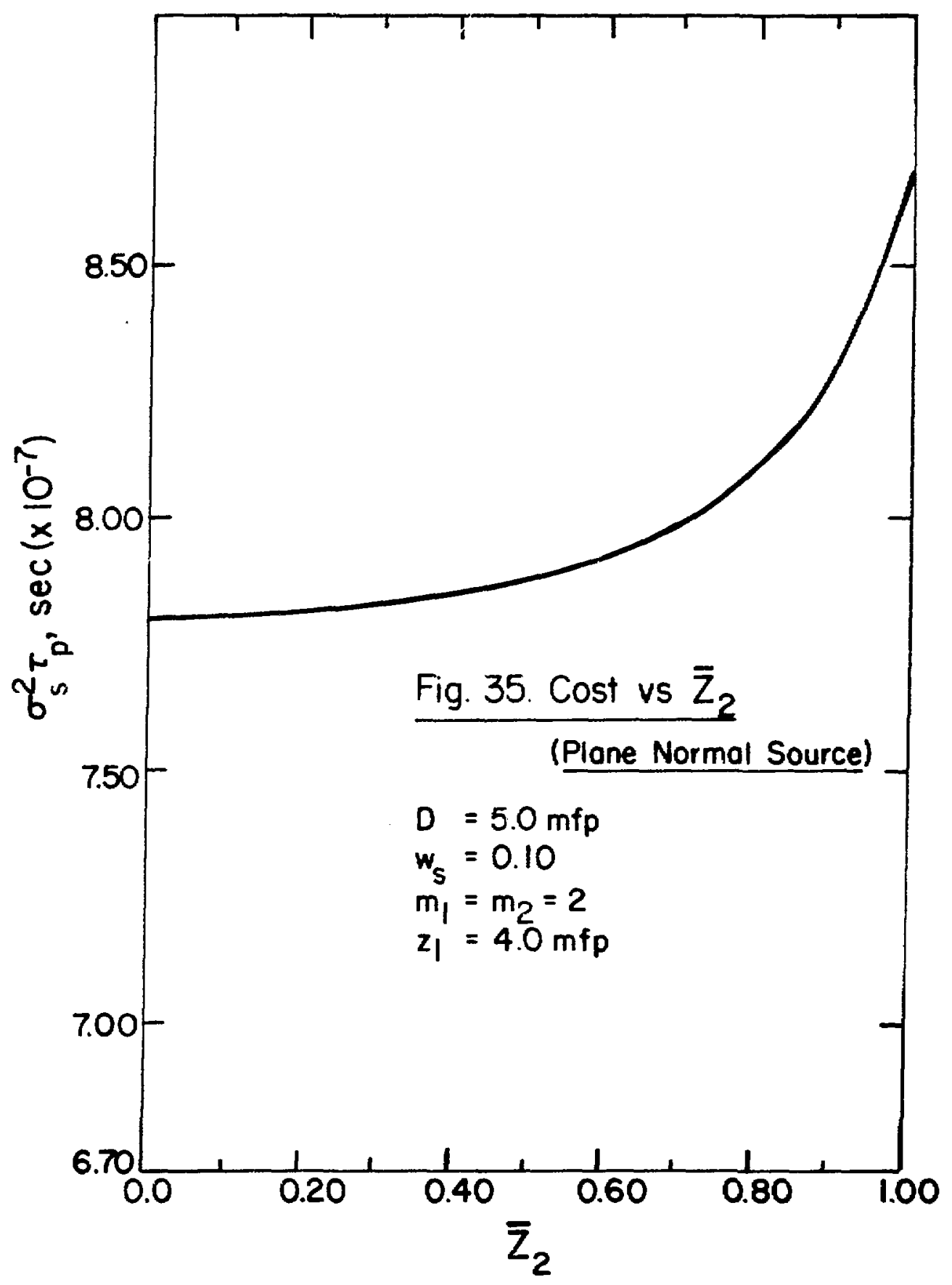




$$
S(0, \mu, w) d \mu= \begin{cases}(1) d \mu & \text { for } 0<\mu \leq 1 \\ 0 & \text { otherwise }\end{cases}
$$

so that $\int_{-1}^{+1} s(0, \mu, w) d \mu=\int_{0}^{1} s(0, \mu, w) d \mu=1$.

Now it is possible to formulate

$$
\begin{aligned}
\mathrm{S}(0, \mu, \mathrm{w}) \psi(0, \mu, w, s) \mathrm{ds} d \mu \equiv & \text { probability that a particle will be } \\
& \text { introduced in d } \mu \text { about } \mu \text { at } z=0 \\
& \text { (with starting weight } w \text { ) and will } \\
& \text { score } s \text { in } d s \text { in the course of its } \\
& \text { subsequent history. }
\end{aligned}
$$

We then multiply the above probability expression by $\mathbf{s}^{r}$ and integrate over ds and du:

$\bar{M}_{r}(0, w) \equiv \int_{0}^{1} d \mu s(0, \mu, w) \int \psi(0, \mu, w, s) s^{r} d s=\int_{0}^{1} M_{r}(0, \mu, w) d \mu \quad \cdot(5.3 .3 .2)$

Thus, $\bar{M}_{r}(0, w)$ represents the $r$-th moment of the Monte Carlo score, given the particle starts out at $z=0$ with weight $w$.

As mentioned in Section 5.3.1, the deterministic $s_{n}$ solution for the $r$-th moment of the Monte Carlo score includes values of $M_{r}$ at $z=0$ for all of the discrete directions $\mu_{\mathrm{m}}$. Thus, by simply performing an integration of the angular solutions over $\mu$ at $z=0, \bar{M}_{r}(0,1)$ may be computed:

$\bar{M}_{r}(0,1)=\int_{0}^{1} M_{r}(0, \mu, 1) d \mu \approx \sum_{m=1}^{M M / 2} M_{r ; m}(0,1) \omega_{m}$, 
where $\mathrm{M}_{\mathrm{r} ; \mathrm{m}}(0,1)$ represents the solution for the $\mathrm{r}-\mathrm{th}$ moment at $\mathrm{z}=0$ along the $m$-th discrete direction $\left(\mu_{m}\right)$, given a starting weight of unity. The summation in Eq. (5.3.3.3) represents the numerical integration of the adjoint $S_{n}$ solution at $z=0$ over all positive directions $\left(\omega_{\mathrm{m}}\right.$ are the quadrature weights associated with an $S_{n}$ calculation employing a quadrature order of $n=M$ ).

The same optimization scheme as used in Section 5.3 .3 a was employed to determine the optimal location of the two splitting surfaces when the source was considered to be isotropic at $z=0$. The numerical integration indicated in Eq. (5.3.3.3) constituted the only extra operation in the procedure.

A $z_{2}$-survey for $z_{1}=2.50 \mathrm{mfp}$ is illustrated in Fig. 36 . The overall optimum splitting surface locations are found to occur at $z_{1(\mathrm{opt})}=2.50 \mathrm{mfp}$ and $z_{2(\mathrm{opt})}=3.58 \mathrm{mfp}\left(\bar{z}_{2(\mathrm{opt})}=0.433\right)$. The attendant minimum cost is $\left(\sigma_{\mathrm{s}}{ }^{2} \tau_{\mathrm{p}}\right)_{\mathrm{MIN}}=7.398 \mathrm{E}-08 \mathrm{sec}$, compared to the no-splitting cost of 1.154 E-07 sec for the isotropic angular source distribution.

It is interesting to note that the optimum splitting surface locations when considering an isotropic (forward-directed) source do not differ by more than 0.1 mean free path from the optimal surface locations attending a plane normal source. This, of course, is not totally surprising in light of the fact that the forward "directional preference" of the plane normal source is lost after one collision in an isotropically scattering medium, i.e., roughly within one mean free path of the source plane. Hence, for a slab thickness of five mean free paths, as considered here, it is not surprising that the optimal locations of 


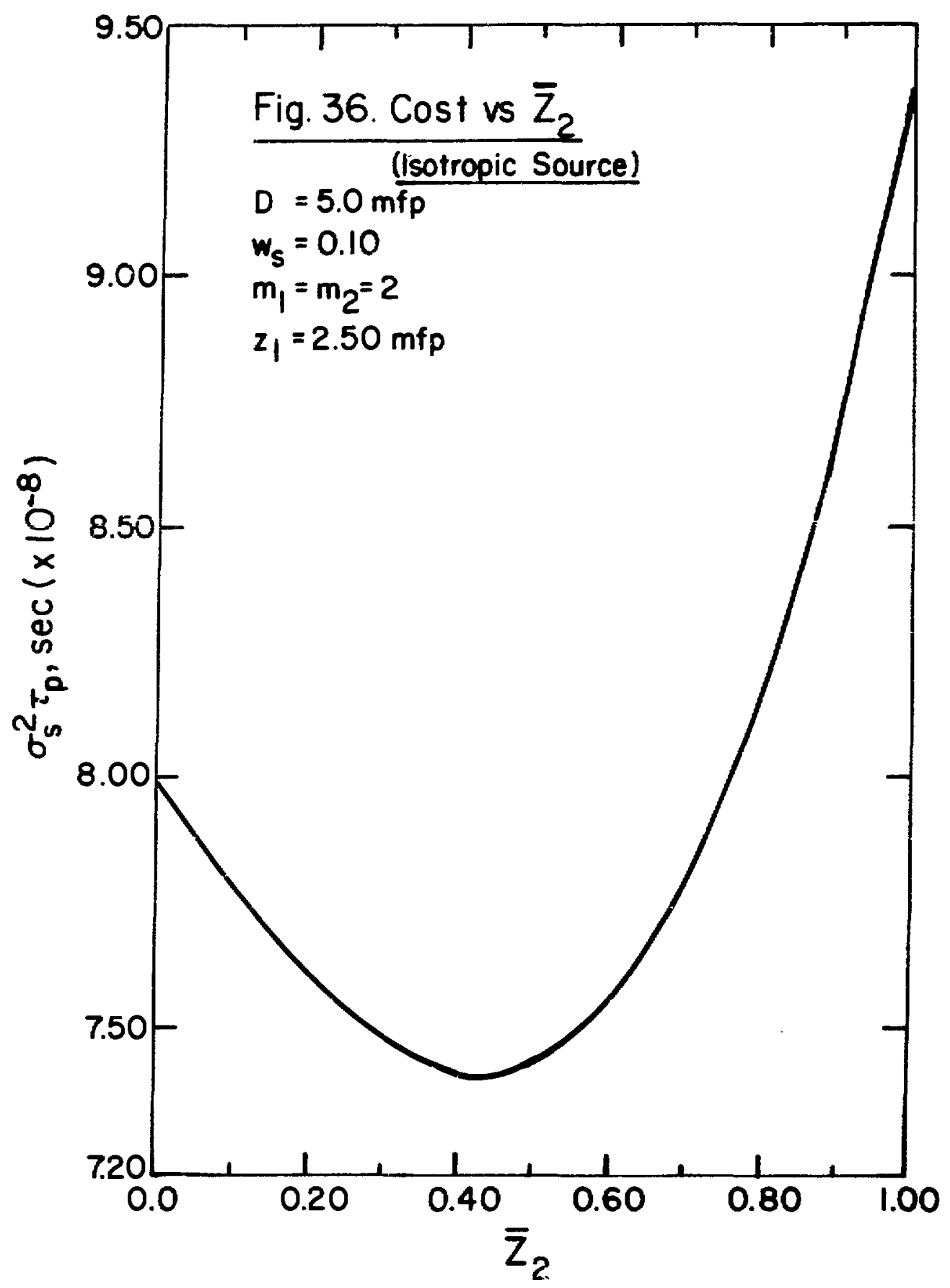

173 
splitting surfaces are not affected much by the angular distribution of the source particles (as long as the medium is isotropically scattering).

\subsection{Comparison of Optimal va. Heuristic Placement of Surfaces.}

The fact that the discontinuity conditions in the solution functions for the second moment of the transmission score and time per particle history generalize for any number of splitting surfaces in the geometry facilitates the prediction of computing costs related to multiple splitting surfaces. It is, therefore, possible to use the appropriate discrete ordinates calculations to deterministically compute the costs associated with multiple surfaces and compare these to optimum single-surface results. In Section 5.3, such analysis was employed to optimize the location of two surfaces. However, costs may also be predicted for cases involving the use of rules of thumb for prescribing splitting parameters.

It was of interest to determine the costs associated with two different heuristics and to compare these with the computing costs related to optimal splitting conditions. One of the heuristic prescriptions involved 2:1 splitting at every mean free path; the other involved 2:1 splitting when the particle flux (not necessarily the track population) diminishes by a factor of 2. The cost comparison was done for a transport problem involving a slab thickness of five mean free paths and a scattering ratio $\left(\Sigma_{\mathrm{S}} / \Sigma_{\mathrm{T}}\right)$ of 0.10 . The source was considered to be normally incident at $z=0$. Results are presented in Table 9.

of the cases considered, the overall optimum splitting condition involves splitting by $4: 1$ at one surface $\left(z_{s}=3.0 \mathrm{mfp}\right)$. Optimum 


\begin{tabular}{|c|c|}
\hline$\underline{\text { CASE }}$ & COST(sec) \\
\hline No splitting & $9.093 \mathrm{E}-7$ \\
\hline $\begin{array}{c}\text { Optimal; one surface } \\
\mathrm{m}=2 \text { at } 3.25 \text { mfp }\end{array}$ & $7.235 \mathrm{E}-7$ \\
\hline $\begin{array}{c}\text { Optimal; one surface } \\
\text { m=4 at 3.00 mfp }\end{array}$ & $6.402 \mathrm{E}-7$ \\
\hline $\begin{array}{c}\text { Optimal; two surfaces } \\
\text { m=2 at 2.50, 3.68 mfp }\end{array}$ & $6.735 \mathrm{E}-7$ \\
\hline $\begin{array}{c}\text { Split by 2 at every } \\
\text { mean free path }\end{array}$ & $7.272 \mathrm{E}-7$ \\
\hline $\begin{array}{c}\text { Split by 2 when } \\
\text { flux falls by } 2\end{array}$ & $9.896 \mathrm{E}-7$ \\
\hline
\end{tabular}

Table 9. Comparison of Several Cases.

[ $D=5.0 \mathrm{mfp} ; \Sigma_{\mathrm{s}} / \Sigma_{\mathrm{T}}=0.10$ ] 
splitting by $2: 1$ at two surfaces $\left(z_{1}=2.50 ; z_{2}=3.68 \mathrm{mfp}\right)$ is only marginally worse. Rule-of-thumb splitting by $2: 1$ at every mean free path is more expensive than the optimal cases, but still cost-effective relative to the non-splitting case. Finally, splitting by $2: 1$ when the particle flux decreases by a factor of 2 is not at alz cost-effective in this case.

Rule-of-thumb splitting by $2: 1$ at every mean free path was investigated somewhat more extensively in an attempt to define better the conditions under which such a splitting scheme would be cost-effective relative to the non-splitting calculation. Several slab thicknesses were considered; for each, calculations were done assining highiyabsorbing as well as highly-scattering media. In all cases, the cost associated with rule-of-thumb splitting was compared to the cost of a non-splitting calculation. Results are summarized in Table 10. Recall that "rule-of-thumb" splitting in Table 10 refers to splitting in a ratio $2: 1$ at every mean free path (corresponding to the total cross section $\Sigma_{\mathrm{T}}$ at the source energy).

The cost comparisons in Table 10 indicate that sucil rule-of-thumb splitting is not effective for thin slabs (approximately less than four mean free paths in thickness), but does provide a cost benefit relative to the non-splitting calculation for moderately-thick and thick slabs only when the transport medium is highly absorbing. In other words, splitting 2:1 at every mean free path is cost-effective only when the transport problem is such that it accommodates, in general, a high degree of splitting, e.g., when considering thick, highly-absorbing slabs. For example, for a slab with a thickness of ten mean free paths and a 


\begin{tabular}{|c|c|c|}
\hline Case & $\begin{array}{r}\operatorname{Cost}, \sigma \\
\text { Rule of Thumb } \\
\end{array}$ & $\begin{array}{l}\text { ) }----- \\
\text { No Splitting }\end{array}$ \\
\hline $\begin{array}{l}\mathrm{D}=3.0 \mathrm{mfp} \\
\mathrm{w}_{\mathrm{s}}=0.10\end{array}$ & $4.234 E-0 E$ & $3.846 \mathrm{E}-06$ \\
\hline $\begin{array}{l}D=3.0 \mathrm{mfp} \\
w_{s}=0.90\end{array}$ & $1.436 \mathrm{E}-05$ & $8.665 \mathrm{E}-\mathrm{G6}$ \\
\hline $\begin{array}{l}\mathrm{D}=5.0 \mathrm{mfp} \\
\mathrm{w}_{\mathrm{s}}=0.10\end{array}$ & $7.272 \mathrm{E}-07$ & $9.093 \mathrm{E}-07$ \\
\hline $\begin{array}{l}\mathrm{D}=5.0 \mathrm{mfp} \\
\mathrm{w}_{\mathrm{s}}=0.70\end{array}$ & $2.035 \mathrm{E}-06$ & $1.635 E-06$ \\
\hline $\begin{array}{l}\mathrm{D}=10.0 \mathrm{mf} p \\
\mathrm{w}_{\mathrm{s}}=0.10\end{array}$ & $3.957 \mathrm{E}-09$ & $1.243 \mathrm{E}-08$ \\
\hline $\begin{array}{l}\mathrm{D}=10.0 \mathrm{mfp} \\
\mathrm{w}_{\mathrm{S}}=0.90\end{array}$ & $1.274 \mathrm{E}-06$ & $3.408 \mathrm{E}-07$ \\
\hline
\end{tabular}

Table 10. Computing Costs Associated with Rule-of-Thumb splitting.

["Rule of Thumb" here refers to 2:1 splitting at every mean free path.] 
scattering ratio of 0.10 , it was found (see Fig. 26 in Chapter IV) that even splitting by 20:1 at one surface provides a significant reduction in cost relative to the non-splitting calculation. Correspondingly, in Table 10 we see that rule-of-thumb splitting for the same problem results in a significant $(68 \%)$ cost benefit. On the other hand, ruleof-thumb splitting results in a $274 \%$ cost penalty when the scattering ratio for the same problem is increased to 0.90 .

The results in Table 9 also allow for a comparison between the cost associated with splitting to a higher degree (e.g., 4:1) at a single splitting surface and the cost of splitting more moderately

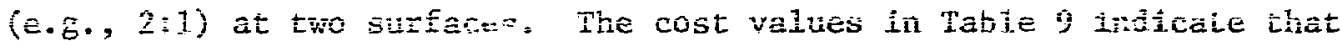
splitting to a higher degree at one surface has a slight advantage. It was of interest to compute such a cost comparison for another transport problem in order to ascertain whether this condition is typical. A slab thickness of ten mean free paths and a medium with a scattering ratio of 0.10 were considered. The cost associated with optimal singlesurface splitting $\left(m=10\right.$ at $\left.z_{s}=6.70 \mathrm{mfp}\right)$ was compared to the cost of splitting more moderately at two surfaces. The two splitting surfaces in the ten mean-free-path slab were located in the same relative locations as two optimally-located surfaces in a five mean-free-path slab (see Section 5.3.3.a). In one case, the splitting ratio at each of the two surfaces was considered to be $m=2$; in the second case the ratio was $\mathrm{m}=5$. The cost comparison is presented in Table 11.

From the results given in Table 11 (which reflect a transport problem calling for a high degree of splitting), it seems that a cost advantage may possibly be gained by splitting in a slightly more 


\begin{tabular}{|c|c|}
\hline$\underline{\text { Case }}$ & Cost (sec) \\
\hline $\begin{array}{l}\text { Optimal one surface; } \\
\mathrm{m}=10 \text { at } 6.70 \mathrm{mf} p .\end{array}$ & $4.359 \mathrm{E}-09$ \\
\hline $\begin{array}{l}\text { Two surfaces; } \\
\mathrm{m}=2 \text { at } 5.0,7.4 \mathrm{mfp} .\end{array}$ & $5.828 \mathrm{E}-09$ \\
\hline $\begin{array}{l}\text { Two surfaces; } \\
\mathrm{m}=5 \text { at } 5.0,7.4 \mathrm{mfp} .\end{array}$ & $3.627 \mathrm{E}-09$ \\
\hline
\end{tabular}

Table 11. Cost Comparison: One vs. Two Splitting Surfaces. $\left(D=10.0 \mathrm{mfp} ; w_{s}=0.10\right)$ 
moderate ratio $(m=5)$ at two surfaces, rather than splitting more severely at only one surface $(m=10)$. Although these few results cannot support a general conclusion, nevertheless the condition stated above seems intuitively plausible. Splitting more moderately at multiple surfaces allows more for keeping the particle track population relatively constant throughout the problem geometry, thus facilitating in the general case a more balanced sampling of all the geometric regions. Splitting to a high degree at only one surface results in a greater discontinuity in the particle track population, possibly involving a greater amount of computer time being spent in tracking particles that do not have a commensurate effect in lowering sample variance. Nevertheless, as indicated in Table 9, for transport problems which do not accommodate a very high degree of splitting, splitting in a moderate ratio at a single surface may still be cost-advantageous vis $a^{\prime}$ vis multiple-surface splitting.

Table 11 also implies that some optimal splitting ratio at the two surfaces most 1ikely exists, since splitting $2: 1$ at each of the surfaces results in an even higher cost (due to undersplitting) than splitting 10:1 at the single surface. The discussion above should serve to indicate that optimum splitting conditions are certainly quite problemdependent.

Needless to say, the few results presented here are in no way intended to provide a definitive solution to the optimal number of splitting surfaces in the geometry (or, for that matter, the optimal degree of splitting at each surface). Such a complete and all-inclusive optimization of splitting strategy is beyond the scope of this 
dissertation. On the other hand, these results demonstrate the usefulness of an analytical tool (such as the one presented in this dissertation) for quantifying deterministically the computational cost associated with the implementation of a popular and important variance reduction device in the Monte Carlo calculation. This type of deterministic analysis constitutes a positive step over arguments based on heuristics or intuition.

\subsection{Comparison to Results from Standard Production Monte Carlo Code (MCNP)}

Throughout this work, the predicted values of computational cost have been constantly compared to actual Monte Carlo results obtained from a special-purpose Monte Carlo code that was written to treat specifically the transport problem which served as a model for this analysis. However, it was also of interest to compare the predicted values of sample variance to stochastic values computed using a standard production Monte Carlo code, ${ }^{9}$ developed at the Los Alamos Scientific Laboratory.

The basic transport problem modeled was the same as before--namely, a semi-infinite slab with a particle source impinging normally on the front surface. Splitting, Russian roulette, and survival blasing were considered. In order to perform the calculation with MCNP, a special cross-section file in ACE format ${ }^{9}$ (the neutron cross section format read by MCNP) had to be constructed. This file contained the special cross sections (constant over all energies) which characterized the transport problem under consideration, i.e., $\Sigma_{T}, \Sigma_{A}$, and $\Sigma_{s}$ with scattering isotropic in the laboratory reference frame. 
Tabie 12 presents the values of expected slab transmission and sample variance for a varlety of transport-problem parameters and splitting conditions. Displayed values include those predicted based on the theory of Monte Carlo errors, as well as stochastic values computed with MCNP. The agreement is quite satisfactory. (The Monte Carlo values of $\sigma_{s}{ }^{2}$ in the table result from calculations of the slab transmission to a relative error of $1 \%$ or better.)

In principle, given the appropriate computer timings for the elementary events of a particle history, it would also be possible to predict the time per particle history for the MCNP Monte Carlo calculation. However, considering the coding complexity of a multi-purpose production code, it was determined that (for the purpose of this work) such a determination of the actual event timings within MCNP would not be an appropriate undertaking. An interesting fact may be stated, however, regarding the overall time required to process a particle history when using MCNP relative to the time per history obtained when employing the special-purpose code (MCSN) used for the stochastic calculations in the major part of this work: the MCNP times were consistently about a factor of ten greater. This brings out the advantage of using a small, specially-written program for a study such as the one involved in this particular work. 


\begin{tabular}{|c|c|c|c|c|}
\hline CASE & & THEORY & MCNP & $z$ DIFF. \\
\hline $\begin{array}{l}D=5.00 \mathrm{mfp} \\
w_{s}=0.50 \\
z_{s}=2.50 \mathrm{mfp} \\
m=2\end{array}$ & $\begin{array}{r}<x> \\
\sigma_{s}^{2}\end{array}$ & $\begin{array}{l}1.294 \mathrm{E}-02 \\
4.785 \mathrm{E}-03\end{array}$ & $\begin{array}{l}1.295 \mathrm{E}-02 \\
4.733 \mathrm{E}-03\end{array}$ & $\begin{array}{l}0.08 \% \\
1.09 \%\end{array}$ \\
\hline $\begin{array}{l}D=5.00 \mathrm{mfp} \\
w_{s}=0.90 \\
z_{s}=1.50 \mathrm{mr} p \\
m=3\end{array}$ & $\begin{array}{r}\langle x\rangle \\
\sigma_{s}^{2}\end{array}$ & $\begin{array}{l}7.667 \mathrm{E}-02 \\
1.61 \mathrm{E}-02\end{array}$ & $\begin{array}{l}7.644 E-02 \\
1.600 E-02\end{array}$ & $\begin{array}{l}0.30 \% \\
0.62 \%\end{array}$ \\
\hline $\begin{array}{l}D=3.00 \mathrm{mfp} \\
w_{s}=0.50 \\
z_{s}=1.25 \mathrm{mfp} \\
m=4\end{array}$ & $\begin{array}{r}<x> \\
\sigma_{s}^{2}\end{array}$ & $\begin{array}{l}7.853 \mathrm{E}-02 \\
2.136 \mathrm{E}-02\end{array}$ & $\begin{array}{l}7.831 E-02 \\
2.112 E-02\end{array}$ & $\begin{array}{l}0.30 \% \\
1.10 \%\end{array}$ \\
\hline $\begin{array}{l}D=1 C .0 \mathrm{mfp} \\
w_{s}=0.90 \\
z_{s}=6.00 \mathrm{mfp} \\
m=3\end{array}$ & $\begin{array}{r}\langle x\rangle \\
\sigma_{s}^{2}\end{array}$ & $\begin{array}{l}5.614 E-03 \\
6.446 E-04\end{array}$ & $\begin{array}{l}5.667 E-03 \\
6.589 E-04\end{array}$ & $\begin{array}{l}0.34 \% \\
2.22 \%\end{array}$ \\
\hline $\begin{array}{l}D=3.00 \mathrm{mfp} \\
w_{s}=0.90 \\
z_{s}=1.50 \mathrm{mfp} \\
m=3\end{array}$ & $\begin{array}{r}\langle x\rangle \\
\sigma_{s}^{2}\end{array}$ & $\begin{array}{l}2.146 \mathrm{E}-01 \\
7.099 \mathrm{E}-02\end{array}$ & $\begin{array}{r}2.154 \mathrm{E}-01 \\
7.120 \mathrm{E}-02\end{array}$ & $\begin{array}{l}0.37 \% \\
0.30 \%\end{array}$ \\
\hline $\begin{array}{l}D=5.00 \mathrm{mf}_{\mathrm{p}} \\
w_{s}=0.10 \\
z_{i} \text { at }\left\{\begin{array}{l}2.00 \mathrm{mfp}_{\mathrm{p}} \\
3.00 \mathrm{mfp}\end{array}\right. \\
\mathrm{m}_{i}=2\end{array}$ & $\begin{array}{r}\langle x\rangle \\
\sigma_{s}^{2}\end{array}$ & $\begin{array}{l}7.319 \mathrm{E}-03 \\
2.086 \mathrm{E}-03\end{array}$ & $\begin{array}{l}7.362 E-03 \\
2.082 E-03\end{array}$ & $\begin{array}{l}0.58 \% \\
0.19 \%\end{array}$ \\
\hline $\begin{array}{l}D=3.00 \mathrm{mfp}_{\mathrm{p}} \\
\mathrm{w}_{\mathrm{s}}=0.10 \\
z_{i} \text { at }\left\{\begin{array}{l}1.00 \mathrm{mfp}_{\mathrm{p}} \\
2.00 \mathrm{mfp}\end{array}\right. \\
\mathrm{m}_{i}=2\end{array}$ & $\begin{array}{r}\langle x\rangle \\
\sigma_{s}^{2}\end{array}$ & $\begin{array}{l}5.300 \mathrm{E}-02 \\
1.831 \mathrm{E}-02\end{array}$ & $\begin{array}{l}5.252 \mathrm{E}-02 \\
1.824 \mathrm{E}-02\end{array}$ & $\begin{array}{l}0.91 \% \\
0.38 \%\end{array}$ \\
\hline $\begin{array}{l}D=5.00 \mathrm{mfp} \\
w_{s}=0.70 \\
z_{1} \text { at }\left\{\begin{array}{l}1.00 \mathrm{mfp} \\
2.00 \mathrm{mfp} \\
3.00 \mathrm{mfp} \\
4.00 \mathrm{mfp}\end{array}\right. \\
m_{i}=2\end{array}$ & $\begin{array}{r}\langle x\rangle \\
\sigma_{s}^{2}\end{array}$ & $\begin{array}{l}2.349 \mathrm{E}-02 \\
2.319 \mathrm{E}-03\end{array}$ & $\begin{array}{l}2.332 \mathrm{E}-02 \\
2.301 \mathrm{E}-03\end{array}$ & $\begin{array}{l}0.72 \% \\
0.78 \%\end{array}$ \\
\hline
\end{tabular}

Table 12. Predicted vs. Stochastic (MCNP) Values of Sample Variance. [ $\langle x\rangle$ denotes expected value of slab transmission probability; splitting surfaces located at $z_{s}$ or $z_{i}$ (multiple).] 
CHAPTER VI

CONCLUSIONS AND RECOMMENDATIONS

\subsection{Conclusions}

The main goal of this research effort, as defined in Chapter I, was successfully met: a deterministic technique was employed in order to predict the efficiency of geometric splitting/Russian roulette in a Monte Carlo calculation. This constitutes a positive step in Monte Carlo methodology, facilitating the effective implementation of a particular variance reduction technique based on quantitative analysis raiher than on guesswork or intuition.

Four major conclusions may be drawn from this work, the last three mainly as a result of the optimization study relating to the single splitting surface:

(1) The theory of Monte Carlo errors may be employed in order to accurately predict the cost associated with a specific set of splitting parameters. The deterministic calculation of the computational cost $\sigma_{s}^{2} \tau_{p}$ allows for an a Eriori optimization of a particular splitting scheme, as well as providing an analytical tool with which to judge the relative merits of various commonly-used heuristics related to splitting.

Several advantages attend the deterministic (in this case $S_{n}$ ) calculations of the cost. First, the solutions to the governing equations, which were in this case adjoint formulations of the Boltzmann transport equation, are global in nature (that is, the solutions to the r-th moments of the Monte Carlo 
score and $\tau_{p}$ are determined for points throughout the geometry and for all discrete directions). Thus, the results of one calculation may be easily integrated over any given source distribution in order to determine $\sigma_{s}^{2} \tau_{p}$ corresponding to that particular distribution. This was demonstrated in Chapter $\mathbf{V}$ for the case of an isotropic planar source. Secondly, the $\mathrm{S}_{\mathbf{n}}$ calculations of the Monte Carlo computational cost were themselves efficient with regard to computing time. On the average, it took approximately 30 seconds of computer time (on the CDC-7600 machine) to generate deterministically a typical $\sigma_{s}^{2} \tau_{p}$ vs. $z_{s}$ survey (for example, one of the curves in Fig. 23). On the other hand, to stochastically verify just one of the points on such a curve using Monte Carlo would require computing time on the order of minutes. (In fact, some of the MCNP results in Table 12 reflect calculations which took about 30 minutes of (DC-7600 time). Finally, the deterministic calculations allow for the prediction of the expected variance as opposed to a stochastic estimate of variance calculated as part of the Monte Carlo calculation and hence subject to statistical fluctuation, i.e., variance of the variance.

(2) The threshold slab thickness for which any splitting (at one surface) becomes cost-effective varies from about two to more than seven mean free paths (based on $\Sigma_{T}$ ), depending on the degree of scattering in the medium. 
The practical implication of this point is obvious: splitting must be used sparingly and with caution in transport calculations involving a high degree of scattering.

(3) When splitting at a single surface is cost-effective, it is so over a wide range of splitting-surface locations.

(4) When splitting at a single surface is cost-effective (especially when the optimal splitting ratio is large), oversplitting and undersplitting by as much as a factor of two will not result in a significant cost penalty (for example, see Fig. 26).

Due to the limited amount of computational support for these next two points, they are stated as observations rather than conclusions. It is possible that more extensive study ensuring their generality (or at least defining conditions under which they hold) may fully substantiate their validity at some future date:

(1) In transport problems which can accommodate a large degree of splitting, e.g., problems allowing for a large splitting ratio at a single surface, a certain cost advantage may posstbly be gained by splitting in a slightly more moderate ratio at two (or possibly more) surfaces rather than splitting more drastically at the single surface. However, the extent of the cosţ advantage will depend heavily on the splitting ratio employed at each of the multiple surfaces. 
(2) Splitting by two at every mean free path (an exande of a common rule-of-thumb splitting prescription) is cost effective, although not necessarily optimum, vis a vis the non-splitting calculation only when implemented in fairly thick slabs which contain a strongly-absorbing medium. In other cases, this splitting strategy may result in expensive oversplitting.

Before discussing recommendations for future work, one more point should be reiterated regarding the transport model used in this study. Although the semi-infinite slab shield represents a fairly uncomplicated geometry, it nevertheless effectively models the condition under which geometric splitting is apt to be extensively applied--namely, in radiation transport problems involving deep penetration. In such Monte Carlo calculations, it is quite advantageous to know how of ten, by how much, and where to split along the straight-line, mean-free-path distance separating the radiation source and tally of interest. In this light, the numerical results presented in this study have practical significance.

\section{2 Recommendations for Future Work}

Although a complete treatment of the topics to be noted here was outside the scope of this particular dissertation, a further investigation of these interesting problems will undoubtedly shed more light on the cost-effectiveness of the technique of splitting in Monte Carlo radiation-transport calculations.

As a result of the calculations done in this study, it was observed that as long as splitting surfaces are not located too close to the 
source or tally, the computing cost $\left(\sigma_{s}{ }^{2} \tau_{p}\right)$ may be more sensitive to the number of such surfaces (and the degree of splitting at each) than to their relative location. Thus, an optimization study involving the number of splitting surfaces and the splitting ratio at each would yield some interesting results. In fact, a more sophisticated optimization technique might be used to define the optimal combination of all the splitting parameters: number and location of surfaces as well as the importances in adjacent geometric regions. An a11-inclusive study, such as the one proposed here, would serve to substantiate more completely the observations made in the previous section. of course, the $S_{n}$ technique for deterministically predicting computing cost would serve very well as an efficient analytical tool for such a study.

In principle, the analysis of splitting costs based on the theory of Monte Carlo errors may be extended in order to treat the more complicated cases of two- or even some three-dimensional geometries. (of course, there is the possibility that the increased computational time attending two- and three-dimensional $s_{n}$ calculations may render such deterministic analyses less advantageous.) In fact, as long as the integral moment equations are capable of being converted to integrodifferential forms which are themselves adjoint formulations of the Boltzmann transport equation, then the effects of geometric splitting/ Russian roulette on $\sigma_{s}^{2} \tau_{p}$ may be assessed for any transport problem capable of being treated by standard $S_{n}$ methods. Certainly transport media characterized by non-isotropic scattering distributions may be considered. Of course, the use of such $S_{n}$ calculations in deterministically predicting $\sigma_{s}{ }^{2} \tau_{p}$ is Inherently limited to the fairly simple 
geometries which are treated by discrete ordinates methods. Nevertheless, even a deterministic analysis based on a more simplified transport model (such as the one considered in this dissertation) may yield some valuable insights (or more enlightened rules of thumb), which could be helpful in implementing splitting in more complicated Monte Carlo problems.

Finally, the theory of Monte Carlo errors may also be employed to study the technique of energy splitting or, for that matter, energy splitting in conjunction with geometric splitting. In a simplistic sense, the problem of slowing down is analogous in the energy domain to deep penetration in the geometric domain. The ultimate goal of splitting in either case is to ensure an appropriate degree of sampling in otherwise undersampled, but nevertheless important, reglons of the problem's phase space. However, implementation of energy splitting is even less amenable to intuitive prescription than is geometric splitting. Significant benefits, then, can result from a deterministic analysis of the computational cost associated with energy splitting in Monte Carlo calculations. 


\section{ACKNOWLEDGMENTS}

I wish to express special appreciation for the guidance and direction provided during the most important stages of this work by the late Dr. J. L. Macdonald, a fine advisor and good friend. It was he who suggested the topic of the research presented in this dissertation.

I also extend special thanks to Prof.. W. R. Johnson, my advisor at the University of Virginia, for his constant guidance and encouragement throughout the endeavor and for his review at Los Alamos of the progress of this work.

I am grateful for the direction of an advisory group at the Los Alamos Scientific Laboratory (LASL) that included Drs. W. L. Thompson, E. D. Cashwe11, T. E. Booth, R. A. Forster, and D. J. Dudziak. This group read the manuscript and offered valuable suggestions. Dr. Cashwell and Dr. Buoth painstakingly reviewed the mathematical details contained in this dissertation, and Dr. Thompson offered valuable and practical guidance in his role as chairman of this group.

The major portion of the research work presented in this dissertation was done in LASL Group $\mathrm{T}-1$, and I am grateful to Dr. D. J. Dudziak for having made this opportunity possible. This dissertation was completed after I joined LASL Group X-6 of which Dr. W. L. Thompson is Group Leader. Other LASL staff were helpful: Drs. W. F. Walters and R. A. Forster provided numerous enlightening discussions, Dr. R. E. Seamon helped me understand the MCNP cross-section format, Mrs. L. L. Peterson provided assistance with illustrations, and Mr. C. A. Painter helped with computer graphics.

I am especially appreciative of the exceptional work of Mrs. Nathana Haines who typed this manuscript.

I am also grateful for the financial aid received from the Associated Western Universities in the form of an AWU-ERDA Laboratory Graduate Participantship (Fellowship) and to the Los Alamos Scientific Laboratory for the two summer Graduate Research Assistantships. 


\section{REFERENCES}

1. Carter, L. L. and E. D. Cashwe11, Particle Transport Simulation with the Monte Carlo Method, ERDA Critical Review Series TID-26607 (1976).

2. Cashwe11, E. D. and C. J. Everett, A Practical Manual on the Monte Carlo Method for Random Walk Problems, Pergamon Press, New York (1959).

3. Spanier, J. and E. M. Gelbard, Monte Carlo Principles and Neutron Transport Problems, Addison Wesley Publ. Co., Reading, Mass. (1969).

4. Clark, M. and K. F. Hansen, Numerical Methods of Reactor Analysis, Academic Press, Inc., New York (1964).

5. Shrieder, Y. A., Ed., The Monte Carlo Method, Pergamon Press, London (1966).

6. Macdonald, J. L. and E. D. Cashwe11, "Application of Artificial Intelligence Techniques to the Acceleration of Monte Carlo Transport Calculations," Los Alamos Scientific Laboratory Report LA-7475-MS (September 1978).

7. Cashwe11, E. D., et al., "MCN: A Neutron Monte Carlo Code," Los Alamos Scientific Laboratory Report LA-4751 (January 1972).

8. Macdonald, J. L., "Investigation of Pattern Recognition Techriques for the Identification of Splitting Surfaces in Monte Carlo Particle Transport Calculations," PhD Thesis, University of Texas at Austin (June 1975); also Los AJamos Scientific Laboratory Report LA-6015-T (August 1975).

9. IASL Group X-6, "MCNP - A General Monte Carlo Code for Neutron and Photon Transport," Los Alamos Sclentific Laboratory Report LA-7396-M (November 1979).

10. Kalos, M. H., F. R. Nakache, and J. Celnick, "Monte Carlo Methods in Reactor Computations," in Computing Methods in Reactor Physics, H. Greenspan, C. N. Kelber, and D. Okrent, Eds., Gordon Breach Publishers, New York (1968).

11. Kahn, H., "Stochastic (Monte Carlo) Attenuation Analysis," Rand Corp. Report P-88 (1949).

12. Everett, C. J. and E. D. Cashwell, "Cost of Splitting in Monte Carlo Transport," Los Alamos Scientific Laboratory Report LA-7189-MS (March 19\%8). 
1.3. Coveyou, R. R., V. R. Cain, and K. J. Yost, "Adjoint and Importance in Monte Carlo Application," Nuc1. Sci. Eng., 27, 219 (1967).

14. An.ter, H. J. and M. J. Djomehri, "Prediction of Statistical Error in Monte Carlo Transport Calculations," Nucl. Sci. Eng., 60, 131 (1976).

15. Booth, T. E. and H. J. Amster, "Prediction of Monte Carlo Errors by a Theory Generalized to Treat Track-Length Estimators," Nucl. Sci. Eng., 65, 273 (1978).

16. Booth, T. E., "Analysis of Error in Monte Carlo Transport Calculations," PhD Thesis, University of Calitornia st Berkeley (October 1978); also Los Alamos Scientific Laboratory Report LA-7636-T (January 1979).

17. Booth, T. E. and E. D. Cashwe11, "Analysis of Error in Monte Carlo Transport Calculations," Nucl. Sci. Eng-, 71, 128 (1979).

18. Sarkar, P. K. and M. A. Prasad, "Prediction of Statistical Error and Optimization of Biased Monte Carlo Transport Calculations," Nuc1. Sci. Eng., 70, 243 (1979).

19. Lux, I., "Systematic Study of Some Standard Variance Reduction Techniques," Nuc1. Sci. Eng., 67, 317 (1978).

20. Be11, G. I. and S. Glasstone, Nuclear Reactor Theory, Von Nostrand Reinhold Co., New York (1970).

21. Hill, T. R., "ONETRAN: A Discrete Ordinates Finite Element Code for the One-Dimensional Multigroup Transport Equation," Los Alamos Scientific Laboratory Report LA-5990-MS (June 1975).

22. Carlson, B. G. and K. D. Lathrop, "Transport Theory - Method of Discrete Ordinates," in Computing Methods in Reactor Physics, H. Greenspan, C. N. Kelber, and D. Okrent, Eds., Gordon Breach Publishers, New York (1968).

23. Lathrop, K. D., "Discrete-Ordinates Methods for the Numerical Solntion of the Transport Equation," React. Tech., 15, 107 (Summer 1972).

24. Abramowitz, M. and I. A. Stegun, Handbook of Mathematical Functions, Dover Publications, Inc., New York (1970).

25. Bevington, P. R., Data Reduction and Error Analysis for the Physical Sciences, McGraw-Hill Book Co., New York (1969). 
APPENDIX A

FORMAL MODLFICATION OF TRANSPORT KERNEL DUE TO SPLITTING

Due to the conceptual simplicity of the geometry splitting technique, its formal characterization as an importance sampling device (in the sense that analog probability densities are weighted by an importance function to produce "fictitious" densities) is elusive. However, Kalos et al. 10 have formally interpreted splitting as a modification of the transport kernel. The argument proceeds with reference to Fig. A-1.

The transport problem to be considered is again particle transmission through a semi-infinite $s l a b$, with particles introduced at the leftmost surface. The slab is divided into laminar importance regions $(n, n+1, n+2$ in Fig. $A-1)$, and the importance function $\left(I_{n}\right)$ is taken to be piecewise constant in these regions. 'It tre transport kernel $\mathrm{C}(\mathrm{s}) \mathrm{ds}$ is defined to be the probability that the particle will undergo its next collision after traversing a distance in ds about s:

$$
C(s) d s=\Sigma_{T}(s) \exp \left[-\int_{0}^{s} \Sigma_{T}\left(s^{\prime}\right) d s^{\prime}\right] d s
$$

Consider a particle starting at the source plane and traveling a distance s; in the course of this flight it intersects the first splitting plane a distance $s_{n}$ from the source. By splitting a particle at this interface into a number of sub-particles $\left(m=I_{n+1} / I_{n}\right)$ and by continuing to track each of the resultant particles from the splitting surface into the $n+1$ 'st region, the net effect is to increase the unweighted 


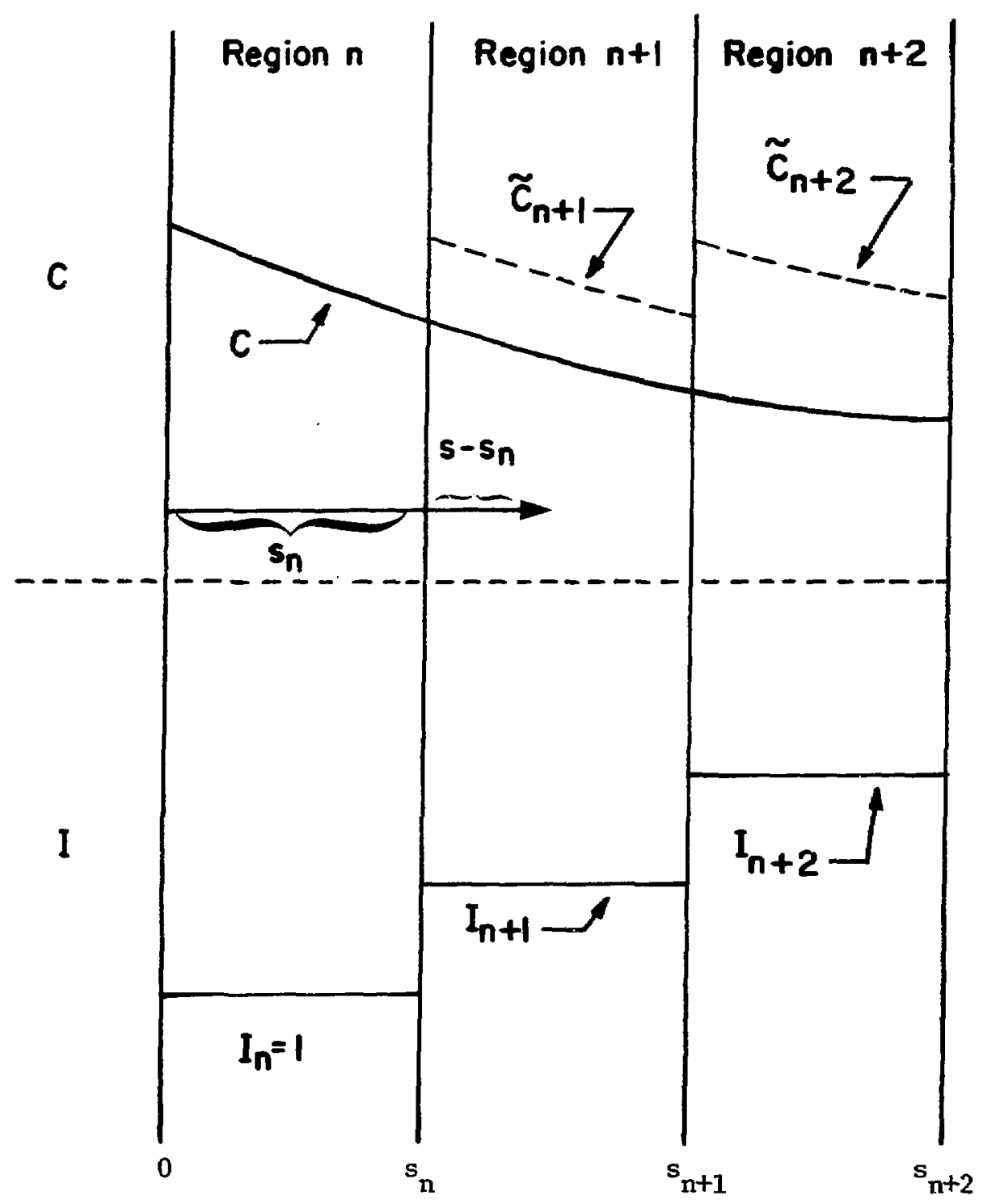

Figure A-1. Modification of Transport Kernel C(s) due to Splitting. 
collision density in region $n+1$ (i.e., per unit source particle). This is reflected as an "apparent" change in the transport kernel. Specifically, by splitting with a factor $I_{n+1} / I_{n}$, the probability of collision in region $n+1$ has been effectively increased by the same factor. Given that the particle proceeds from the splitting surface:

$$
\widetilde{c}_{n+1}\left(s-s_{n}\right) d s=\left(\frac{I_{n+1}}{I_{n}}\right) c\left(s-s_{n}\right) d s .
$$

If the particle starts out from the source,

$\widetilde{c}_{n+1}(s)=\exp \left[-\int_{0}^{s_{n}} \Sigma_{T}\left(s^{-\rho}\right) d s^{-}-\left(\frac{I_{n+1}}{I_{n}}\right) \exp \left[-\int_{0}^{s-s_{n}} \Sigma_{T}\left(s^{\prime}\right) d s^{\prime}\right] \Sigma_{T}\left(s^{\prime}\right)\right.$,

or $\left(\right.$ for $\left.s_{n}<s<s_{n+1}\right)$ :

$$
\tilde{c}_{n+1}(s) d s^{\prime}=\left(\frac{I_{n+1}}{I_{n}}\right) \Sigma_{T}(s) \exp \left[-\int_{0}^{s} \Sigma_{T}\left(s^{\prime}\right) d s^{\prime}\right] d s .
$$

Comparing Eq. (A.4) and (A.1), we find that

$$
\widetilde{c}_{n+1}(s) d s=\left(\frac{I_{n+1}}{I_{n}}\right) c(s) d s \text {, for } s_{n}<s<s_{n+1} \text {. }
$$

Thus, the process of splitting effectively results in the sampling of a kernel that has been weighted by the importance function, corresponding to the formal definition of importance sampling. Note the relative 
magnitudes of the biased transport kernels in the various importance regions, compared to the unaltered (analog) kernel (Fig. A-1). The effect of splitting is to faciljtate a decper penetration of the shield than would be possible if a strictly analog process were considered. 


\begin{abstract}
APPENDIX B
DERIVATION OF THE INTEGRAL MOMENT EQUATIONS FOR THE

MONTE CARLO SLAB TRANSMISSION SCORE (SINGLE SPLITTING SURFACE)
\end{abstract}

The development of the integral equations governing the $r$-th moment of the Monte Carlo score is presented here in some detail. Specifically, the formulations for the first two moments of the slab transmission score are derived in the first section of this Appendix. The second section is devoted to the characterization of the solution functions at the splitting surface, i.e., continuity in the first moment solution and discontinuity in the second.

\title{
B.1 The Integral Moment Equations ( $r=1,2)$
}

The moment equations must be written for three cases, depending on the starting location of the particle relative to the splitting surface. First, for the case of particles starting to the left of the splitting surface $\left(0 \leq z<z_{s}\right)$, Eq. (4.1.1.1) is multiplied by $s^{r}$ and integrated over ds to yield the $r$-th moment of the score:

$$
M_{r, 1} \equiv \int \psi_{1}(\underline{P}, s) s^{r} d s \text { for } 0 \leq z<z_{s} \text {. }
$$

In performing the integration indicated in Eq. (B.I.1) a change of variables is employed which facilitates the treatment of terms in which a Iinear combination of scores appears in the argument of the $\psi$ function. For example, the substitution $s^{*}=s-s^{-}$is made, and the Binomial Theorem is applied to express

$$
\left(s^{*}+s^{\prime}\right)^{r}=\sum_{n=0}^{r}\left(\begin{array}{l}
r \\
n
\end{array}\right) s^{-r-n} s^{* n}
$$


The following integration is then carried out in the scattering term [using Eq. (B.1.2a)]:

$$
\begin{aligned}
\int \psi_{1}\left(\underline{p}^{\prime}, s-s^{\prime}\right) s^{r} d s & =\int \psi_{1}\left(\underline{P}^{\mu}, s^{*}\right)\left(s^{*}+s^{\prime}\right)^{r} d s^{*} \\
& =\sum_{n=0}^{r}\left(\begin{array}{l}
r \\
n
\end{array}\right) s^{-r-n} \int \psi_{1}\left(\underline{P}^{-\rho}, s^{*}\right) s^{*} n d s^{*} \\
& \left.=\sum_{n=0}^{r}\left(\begin{array}{l}
r \\
n
\end{array}\right) s^{-r-n} M_{n, 1} \underline{P}^{-\rho}\right)
\end{aligned}
$$

The above procedure is also applied in times in integrating the splitting term of Eq. (4.1.1.1). If the substitution $s^{*}=s-\left(s^{*}+s_{1}+\cdots+s_{m-1}\right)$ is made, then the integration over as is first carried out, giving

$$
\begin{aligned}
\int \dot{\psi}_{z_{s}}\left(\underline{p}^{\prime \prime}, s\right. & \left.-\left(s^{\prime}+s_{1}+\cdots+s_{m-1}\right)\right) s^{r} d s \\
& =\sum_{n_{1}=0}^{r}\left(\begin{array}{l}
r \\
n_{1}
\end{array}\right)\left(s^{\prime}+s_{1}+\cdots+s_{m-1}\right)^{r-n_{1}} \int_{z_{s}}\left(\underline{P}^{\prime \prime}, s^{*}\right) s^{* n_{1}} d s^{*} \\
& \left.=\sum_{n_{1}=0}^{r}\left(\begin{array}{l}
r \\
n_{1}
\end{array}\right) M_{n_{1}, z_{s}} \underline{p}^{\prime \prime}\right)\left(s_{m-1}+s^{\prime}+s_{1}+\cdots+s_{m-2}\right)^{r-n_{1}}
\end{aligned}
$$

By expressing

$$
\begin{gathered}
\left(s_{m-1}+s^{\prime}+s_{1}+\cdots+s_{m-2}\right)^{r-n_{1}}=\sum_{n_{2}=6}^{r-n_{1}}\left(\begin{array}{c}
r-n_{1} \\
n_{2}
\end{array}\right) \\
\quad \times\left(s^{-}+s_{1}+\cdots+s_{m-2}\right)^{r-n_{1}-n_{2}}{ }_{s_{m-1}}^{n_{2}},
\end{gathered}
$$


the next integration is facilitated:

$$
\begin{aligned}
\int \psi_{z_{s}} & \left(\underline{P}^{\prime \prime}, s_{m-1}\right)\left(s_{m-1}+s^{\prime}+s_{1}+\cdots+s_{m-2}\right)^{r-n_{1}} d s_{m-1} \\
& =\sum_{n_{2}=0}^{r-n_{1}}\left(\begin{array}{c}
r-n_{1} \\
n_{2}
\end{array}\right) M_{n_{2}, z_{s}}\left(\underline{P}^{\prime \prime}\right)\left(s_{m-2}+s^{\prime}+s_{1}+\cdots+s_{m-3}\right)^{r-n_{1}-n_{2}} .
\end{aligned}
$$

The procedure is repeated $m-2$ more times in similar fashion. After performing these integrations, the explicit form of Eq. (B.1.1) is written [using Eq. (4.1.1.1)]:

$$
\begin{aligned}
& M_{r, 1}(\underline{P})=\int_{0<z} d_{\underline{P}^{+}<z}^{+} T\left(\underline{P}, \underline{\underline{P}}^{+}\right) \int \underline{\mathrm{P}}^{-} \sigma\left(\underline{\underline{P}}^{+}, \mathrm{P}^{\prime}\right) \int \mathrm{ds^{ \prime }} \mathrm{p}_{\mathrm{S}}\left(\underline{\underline{P}}^{\prime}, \mathrm{s}^{\prime}\right) \int \mathrm{d \underline {P } ^ { \prime }} \\
& \times\left[E\left(\underline{P}^{\prime}, \underline{P}^{-\prime}\right) \sum_{n=0}^{r}\left(\begin{array}{l}
r \\
n
\end{array}\right) M_{n, 1}\left(\underline{P}^{-\rho}\right) s^{-r-n}\right]+\int d \underline{P}^{+} D_{0}\left(\underline{P}, \underline{P}^{+}\right) \int d s
\end{aligned}
$$

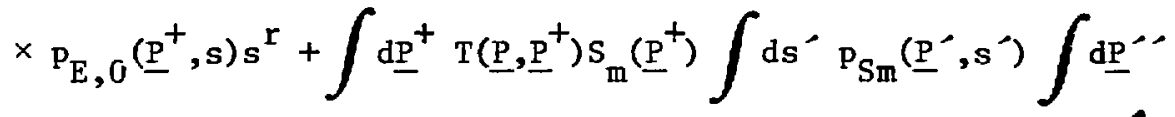

$$
\begin{aligned}
& \times\left[B_{S m}\left(\underline{P}^{+}, \underline{P}^{\prime \prime}\right) \sum_{n_{1}=0}^{r}\left(\begin{array}{l}
r \\
n_{1}
\end{array}\right) M_{n_{1}, z_{s}}\left(\underline{P}^{\prime \prime}\right) \sum_{n_{2}=0}^{r-n_{1}}\left(\begin{array}{c}
r-n_{1} \\
n_{2}
\end{array}\right) M_{n_{2}, z_{s}}\left(\underline{P}^{\cdots}\right) \cdots\right. \\
& \left.\cdots \sum_{n_{m}=0}^{r-n_{1} \cdots \cdots-n_{m-1}}\left(\begin{array}{c}
r-n_{1} \cdots-n_{m-1} \\
n_{m}
\end{array}\right)_{M_{m}, z}\left(\underline{p}^{-\rho}\right) s^{-r-n_{1}-n_{2} \cdots-n_{m}}\right]
\end{aligned}
$$

A similar multiplication of Eq. (4.1.1.2) by $s^{r}$ and integration over ds yields the $r$-th moment in the second case: 


$$
M_{r, 2}(\underline{P}) \equiv \int \psi_{2}(\underline{P}, s) s^{r} d s \text { for } z_{s}<z \leq D \text {. }
$$

More explicitly,

$$
\begin{aligned}
& M_{\mathrm{r}, 2}(\underline{\mathrm{P}})=\int_{z_{\mathrm{s}} \mathrm{z}^{+}<\mathrm{D}} \underline{\mathrm{P}}^{+} \mathrm{T}\left(\underline{\mathrm{P}}, \underline{\mathrm{P}}^{+}\right) \int \mathrm{dP}^{\prime} \sigma\left(\underline{\mathrm{P}}^{+}, \underline{\mathrm{P}}^{\prime}\right) \int \mathrm{ds^{ \prime }} \mathrm{P}_{\mathrm{S}}\left(\underline{\mathrm{P}}^{\prime}, \mathrm{s}^{\prime}\right) \int \mathrm{d \underline {P } ^ { \prime }} \\
& \left.\times\left[E\left(\underline{P}^{\prime}, \underline{P}^{-\rho}\right) \sum_{n=0}^{r}\left(\begin{array}{l}
r \\
n
\end{array}\right) M_{n, 2} \underline{P}^{-\rho}\right) S^{-r-n}\right]+\int d \underline{P}^{+} D_{T}\left(\underline{P}, \underline{P}^{+}\right) \int d s \\
& \times p_{E, D}\left(\underline{P}^{+}, s\right) s^{r}+\int d \underline{P}^{+} T\left(\underline{P}, \underline{P}^{+}\right) R_{m}\left(\underline{P}^{+}\right) \int d s^{-} p_{R}\left(\underline{P}^{+}, s^{\prime}\right) \int d \underline{P}^{-} \\
& \times\left[B_{0 m}\left(\xi_{m} ; \underline{p}^{+}, \underline{p}^{\prime \prime}\right) \sum_{n=0}^{r}\left(\begin{array}{l}
r \\
n
\end{array}\right) M_{n, z}\left(\underline{p}^{\prime \prime}\right) s^{-r-n}\right] \text {. }
\end{aligned}
$$

Finally, for the case of particles starting at the splitting surface [see Eq. $(4.1 .1 .3)$ ],

$$
\begin{aligned}
& M_{r, z}(\underline{P}) \equiv \int \psi_{z_{s}}(\underline{P}, s) s^{r} d s \text { for } z=z_{s} \text {, or }
\end{aligned}
$$

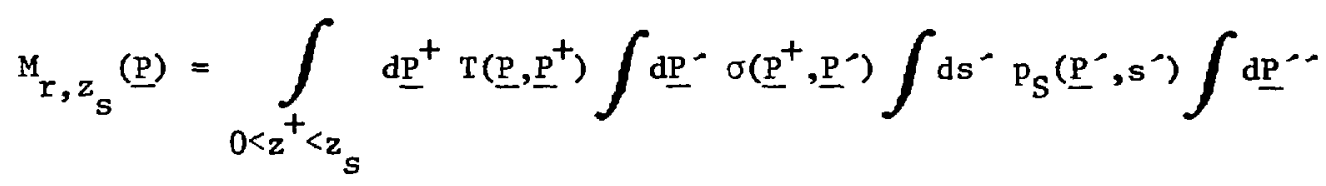

$$
\begin{aligned}
& \times\left[E\left(\underline{P}^{-}, \underline{\underline{P}}^{-\rho}\right) \sum_{n=0}^{r}\left(\begin{array}{l}
r \\
n
\end{array}\right) M_{n, 1}\left(\underline{P}^{\prime \prime}\right) s^{-r-n}\right]+\int_{z_{s}<z^{+}<D} d \underline{P}^{+} T\left(\underline{P}, \underline{P}^{+}\right) / \underline{P}^{-}
\end{aligned}
$$

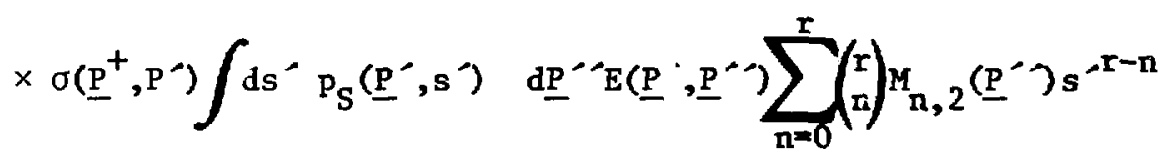

$$
\begin{aligned}
& +\int \underline{\mathrm{P}}^{+} \mathrm{D}_{0}\left(\underline{\mathrm{P}}, \underline{\mathrm{P}}^{+}\right) \int \mathrm{ds} \mathrm{P}_{\mathrm{E}, 0}\left(\underline{\mathrm{P}}^{+}, \mathrm{s}\right) \mathrm{s}^{r}+\int \mathrm{dP}^{+} \mathrm{D}_{\mathrm{T}}\left(\underline{\mathrm{P}}, \underline{\mathrm{P}}^{+}\right) \int \mathrm{ds} \mathrm{P}_{\mathrm{E}, \mathrm{D}}\left(\underline{\mathrm{P}}^{+}, \mathrm{s}\right) \mathrm{s}^{\mathrm{r}} \text {. }
\end{aligned}
$$


The next step in this development is to collect all terms with $M_{r}$ in Eqs. (B.1.4), (B.1.6), and (B.1.8); all non-r-th moment terms in each equation are included in a "source-like" term $Q_{r}$. In performing this separation, a special note should be made regarding the sequence of summations in the splitting term of Eq. (B.1.4). There are exactly $m$ terms in this nested summation which consist only of $M_{r, z_{S}}\left(\underline{P}^{\prime \prime}\right):$ one for $n_{1}=r$ and all other $n_{i}(i \neq 1)=0$, one for $n_{2}=r$ and all other $n_{i}(i \neq 2)=0$, etc.; hence, the term involving $\mathrm{mM}_{r, z_{S}}$ in Eq. (B.1.11). Tise is also made of the fact that

$$
\mathrm{M}_{0}\left(\underline{\mathrm{P}}^{\prime}\right) \equiv \int \psi\left(\underline{\mathrm{P}}^{\prime}, \mathrm{s}\right) \mathrm{s}^{0} \mathrm{ds}=1 \text {, }
$$

since $\psi$ is a probability density function.

A1so, in writing the next few equations, 1.e., Eqs. (B.1.11), (B.1.12), and (B.1.13), integrations over the score distribution functions are evaluated where appropriate. Since these distributions are also probability densities,

$$
\int p_{i}\left(\underline{P}^{\prime}, s^{\prime}\right) d s^{\prime}=1
$$

there the subscript $i$ denotes a particular event. Thus, after collecting all $\mathrm{r}$-th moment terms, the equations are expressed as 


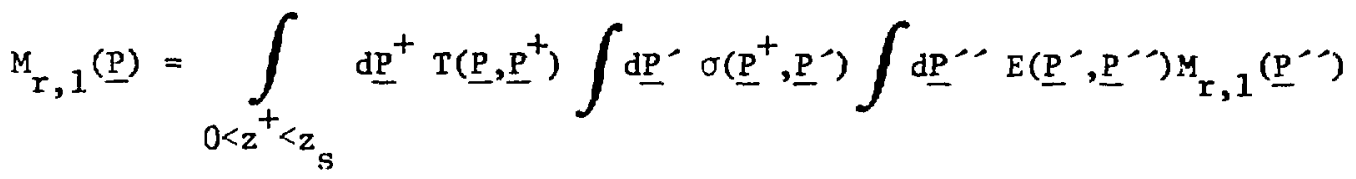

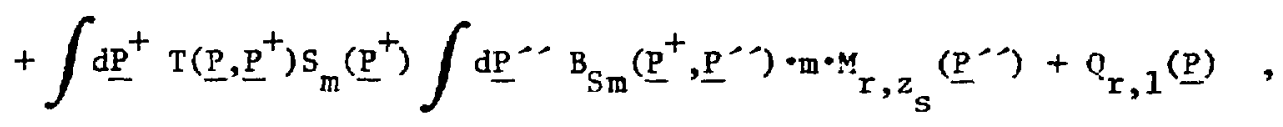

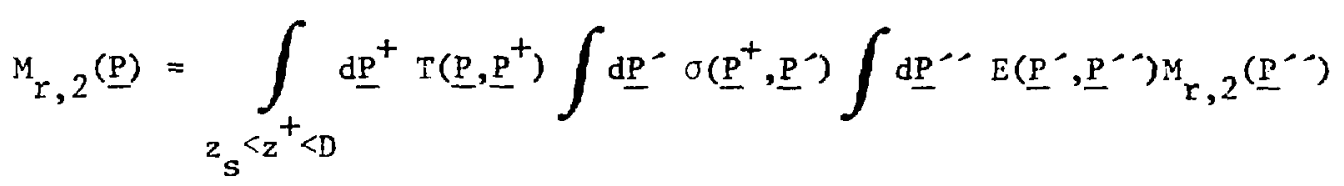

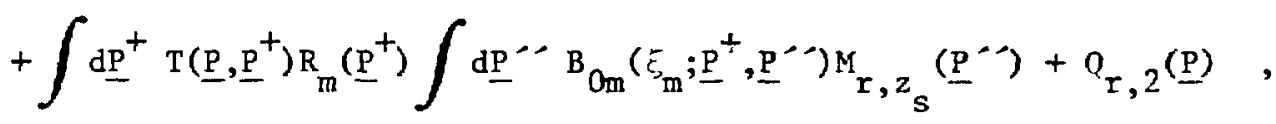

and

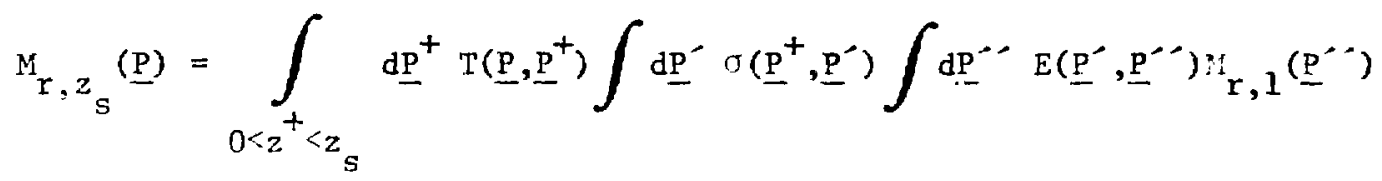

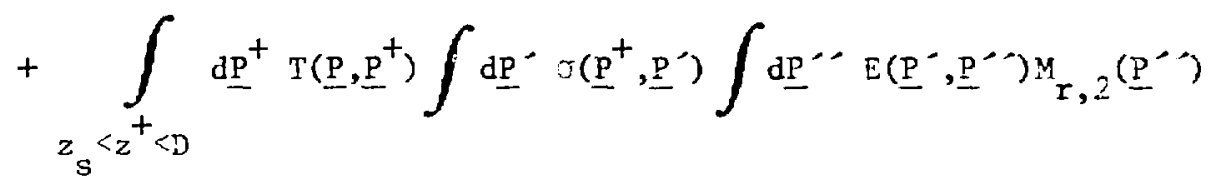

$$
\begin{aligned}
& +Q_{r, z_{s}}(\underline{P)} .
\end{aligned}
$$

The source terms $Q_{r}$ appearing in the above equations are expiicicly written as follows (noting that the dumy integration variable $s^{-}$is replaced by s): 


$$
\begin{aligned}
& Q_{r, 1}(\underline{P})=\int_{0, z^{+}<z} d \underline{P}^{+} T\left(\underline{P}, \underline{P}^{+}\right) \int d \underline{P}^{-} \sigma\left(\underline{P}^{+}, \underline{P}^{\prime}\right) \int d s p_{s}\left(\underline{P}^{-}, s\right) \int d \underline{p}^{-} \\
& \times\left[E\left(\underline{P}^{\prime}, \underline{P}^{\prime}\right) \sum_{n=0}^{r-1}\left(\begin{array}{l}
r \\
n
\end{array}\right) M_{n, 1}\left(\underline{P}^{-\cdots}\right) s^{r-n}\right]+\int d \underline{P}^{+} D_{0}\left(\underline{P}, \underline{P}^{+}\right) \int d s p_{E, 0}\left(\underline{P}^{+}, s\right) s^{r} \\
& +\int d \underline{P}^{+} \mathrm{T}\left(\underline{\mathrm{P}}, \underline{\mathrm{P}}^{+}\right) \mathrm{S}_{\mathrm{m}}\left(\underline{\mathrm{P}}^{+}\right) \int \mathrm{ds} \mathrm{p}_{\mathrm{Sm}}\left(\underline{\mathrm{P}}^{+}, \mathrm{s}\right) \int \mathrm{d} \underline{\mathrm{P}}^{-} \mathrm{B}_{\mathrm{Sm}}\left(\underline{\mathrm{P}}^{+}, \underline{\mathrm{P}}^{-\rho}\right) \sum_{\mathrm{n}_{1}=0}^{\mathrm{r}}\left(\begin{array}{l}
\mathrm{r} \\
\mathrm{n}_{1}
\end{array}\right) \\
& \times M_{n_{1}, z}\left(\underline{P}^{-\prime}\right) \sum_{n_{2}=0}^{r-n_{1}}\left(\begin{array}{c}
r-n_{1} \\
n_{2}
\end{array}\right) M_{n_{2}, z}\left(\underline{P}^{-\mu}\right) \cdots \sum_{n_{m}=0}^{r-n_{1}-\cdots-n_{m-1}} \\
& \times\left(\begin{array}{c}
r-n_{1}-\cdots-n_{m-1} \\
n_{m}
\end{array}\right) M_{n_{m}, z_{s}} \underline{(P}^{-\cdots} s^{r-n_{1}-\cdots-n_{m}} \prod_{i=1}^{m}\left(1-\delta_{n_{i}, r}\right),(B .1 .14)
\end{aligned}
$$

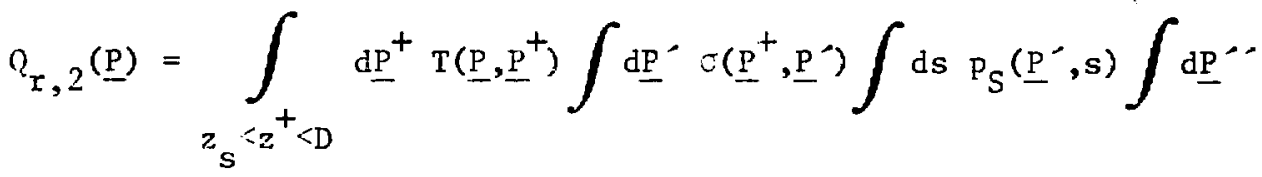

$$
\begin{aligned}
& \times\left[E\left(\underline{P}^{\prime}, \underline{P}^{-\prime}\right) \sum_{n=0}^{r-1}\left(\begin{array}{l}
r \\
n
\end{array}\right) M_{n, 2}\left(\underline{P}^{-\mu}\right) s^{r-n}\right]+\int d \underline{P}^{+} D_{T}\left(\underline{P}, \underline{P}^{+}\right) \int d s p_{E, D}\left(\underline{P}^{+}, s\right)
\end{aligned}
$$

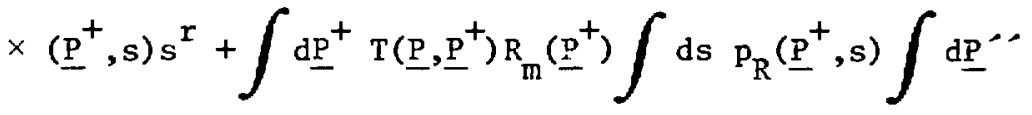

$$
\begin{aligned}
& \times B_{0 m}\left(\xi_{m} ; \underline{P}^{+}, \underline{P}^{-}\right) \sum_{n=0}^{r-1}\left(\begin{array}{l}
r \\
n
\end{array}\right) M_{n, z}\left(\underline{P}^{-}\right) s^{r-n},
\end{aligned}
$$




$$
\begin{aligned}
& Q_{r, z}(\underline{P})=\int_{0<z^{+}<z_{s}} d \underline{P}^{+} T\left(\underline{P}, \underline{P}^{+}\right) \int d \underline{P}^{-} \sigma\left(\underline{P}^{+}, \underline{P}^{\prime}\right) \int d s p_{S}\left(\underline{P}^{-}, s\right) \int \underline{P}^{\prime} \\
& \times E\left(\underline{P}^{\prime}, \underline{P}^{\prime \prime}\right) \sum_{n=0}^{r-1}\left(\begin{array}{l}
r \\
n
\end{array}\right) M_{n, 1}\left(\underline{P}^{-\prime}\right) s^{r-n}+\int_{z_{s}<z^{+}<D} d \underline{P}^{+} T\left(\underline{P}, \underline{P}^{+}\right) \int d \underline{P}^{-} \\
& \left.\times \sigma\left(\underline{\underline{P}}^{+}, \underline{\mathrm{P}}^{-}\right) \int \mathrm{d} s \mathrm{P}_{\mathrm{S}}\left(\underline{\mathrm{P}}^{-}, \mathrm{s}\right) \int \mathrm{d} \underline{\mathrm{P}}^{\cdots} \mathrm{E}\left(\underline{\mathrm{P}}^{-}, \underline{\mathrm{P}}^{-}\right) \sum_{n=0}^{\mathrm{r}-1}\left(\begin{array}{l}
\mathrm{r} \\
\mathrm{n}
\end{array}\right) \mathrm{M}_{n, 2} \underline{\underline{\mathrm{P}}}^{-\rho}\right) \mathrm{s}^{\mathrm{r}-\mathrm{n}} \\
& +\int d \underline{P}^{+} D_{0}\left(\underline{P}, \underline{P}^{+}\right) \int d s p_{E, 0}\left(\underline{P}^{+}, s\right) s^{r}+\int d \underline{P}^{+} D_{T}\left(\underline{P}, \underline{P}^{+}\right) \int d s \\
& \times p_{E, D}\left(P^{+}, s\right) s^{r} .
\end{aligned}
$$

The Kroenecker delta $\delta_{n_{i}, r}$ is used in Eq. (B.1.14) to exclude all the $M_{r}$ terms which already appear in Eq. $(B .1 .11)\left(\delta_{n_{i}, r}=1\right.$ if $n_{i}=r$; $\delta_{n_{i}, r}=0$ otherwise).

From this point on, attention will be specifically directed only to the first and second moments $(r=1$ and 2$)$, since only these are required in expressing the sample variance, c.f. Eq. (1.2.3). The source terms $Q_{r, 1}, Q_{r, 2}$, and $Q_{r, z}$ will now be specifically written for $r=1$ and $r=2$. However, in order to do so, a few identities are stated first.

Since non-analog survival biasing is assumed for the Monte Carlo transport problem under consideration, a particle always exits a collision. This implies that

$$
\int E\left(\underline{P}^{\prime}, \underline{P}^{-\prime}\right) d \underline{P}^{\prime \prime}=1
$$


Also from the definitions stated at the beginning of Chapter IV, it is evident that

$$
\int \mathrm{B}_{0 \mathrm{~m}}\left(\xi_{\mathrm{m}} ; \underline{\mathrm{P}}^{+}, \underline{\mathrm{P}}^{\cdots}\right) \mathrm{d} \underline{\mathrm{P}}^{\cdots}=1
$$

and $\int \mathrm{B}_{\mathrm{Sm}_{\mathrm{m}}}\left(\underline{\mathrm{p}}^{+}, \underline{\mathrm{p}}^{-\rho}\right) \underline{\mathrm{p}}^{\cdots}=1$.

Booth (see Ref. 16, pp. 41-44) has shown that the summation appearing in the last term of Eq. (B.1.14) may be simplified for $r=2$ as follows:

$$
\begin{aligned}
& \left.\sum_{n_{1}=0}^{2}\left(\begin{array}{l}
2 \\
n_{1}
\end{array}\right) M_{n_{1}, z_{s}}\left(\underline{P}^{\cdots}\right) \cdots \sum_{n_{m}=0}^{2-n_{1} \cdots-n_{m-1}}\left(\begin{array}{c}
2-n_{1}-\cdots-n_{m-1} \\
n_{m}
\end{array}\right) M_{n_{m}, z_{s}} \underline{P}^{\cdots}\right) \\
& \times s^{2-n_{1}-\cdots-n_{m}} \prod_{i=1}^{m}\left(1-\delta_{n_{i}, 2}\right)=s^{2}+2 s m M_{1, z}\left(\underline{P}^{\cdots}\right) \\
& +m(m-1)\left[M_{1, z_{s}}\left(\underline{P}^{\cdots}\right)\right]^{2}
\end{aligned}
$$

This facilitates the simplification of $Q_{2,1}$.

By employing the identities stated in Eqs. (B.1.17), (B.1.18), and (B.1.19) [as well as Eq. (B.1.9)], the source terms $Q_{r}$ may be simplified for $r=1$ and 2 . Thus, for particles with starting points $\underline{\mathrm{P}}$ such that $0 \leq \mathrm{z}<\mathrm{z}$,

$$
\begin{aligned}
& \left.Q_{1,1}(\underline{P})=\int_{0<z^{+}<z_{s}} d \underline{\mathrm{P}}^{+} \mathrm{T}\left(\underline{\mathrm{P}}, \underline{\mathrm{P}}^{+}\right) \int \underline{\mathrm{P}}^{-} \sigma\left(\underline{\mathrm{P}}^{+}, \underline{\mathrm{P}}^{\prime}\right) \int \mathrm{ds} \underline{\mathrm{p}}_{\mathrm{S}} \underline{\underline{\mathrm{P}}}^{-}, \mathrm{s}\right) \mathrm{s}+\int \mathrm{d \underline { \textrm {P } } ^ { + }} \\
& \times D_{0}\left(\underline{P}, \underline{P}^{+}\right) \int \mathrm{d} s \mathrm{P}_{\mathrm{E}, 0}\left(\underline{\mathrm{P}}^{+}, s\right) s+\int \mathrm{d} \underline{\mathrm{P}}^{+} \mathrm{T}\left(\underline{\mathrm{P}}, \underline{\underline{P}}^{+}\right) \mathrm{s}_{\mathrm{m}}\left(\underline{\mathrm{P}}^{+}\right) \int \mathrm{ds} \mathrm{P}_{\mathrm{Sm}}\left(\underline{\mathrm{P}}^{+}, s\right) \mathrm{s}
\end{aligned}
$$




$$
\begin{aligned}
& \text { for } r=1 \text {, and }
\end{aligned}
$$

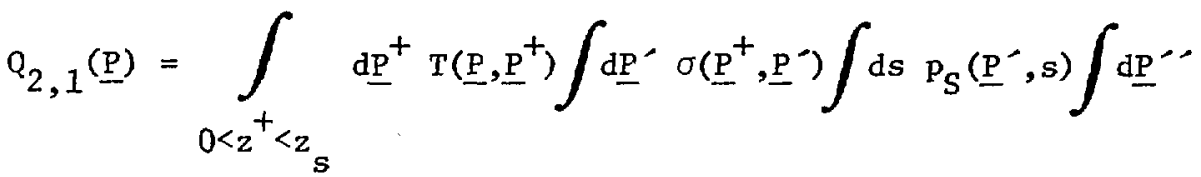

$$
\begin{aligned}
& \times\left[E\left(\underline{P}^{-}, \underline{p}^{\cdots}\right) \sum_{n=0}^{1}\left(\begin{array}{l}
2 \\
n
\end{array}\right) M_{n, 1}\left(\underline{P}^{-\cdots}\right) s^{2-n}\right]+\int d \underline{P}^{+} D_{0}\left(\underline{P}, \underline{P}^{+}\right) \int d s p_{E, 0}\left(\underline{P}^{+}, s\right) s^{2} \\
& +\int \underline{d P}^{+} T\left(\underline{P}, \underline{P}^{+}\right) S_{m}\left(\underline{P}^{+}\right) \int \mathrm{ds} \mathrm{P}_{\mathrm{Sm}}\left(\underline{\mathrm{P}}^{+}, \mathrm{s}\right) \int \mathrm{d} \underline{\mathrm{P}}^{-} \mathrm{B}_{\mathrm{Sm}}\left(\underline{\mathrm{P}}^{+}, \underline{\mathrm{P}}^{-}\right) \\
& \times\left[s^{2}+2 s m M_{1, z}\left(\underline{P}^{\infty}\right)+m(m-1)\left\{M_{1, z}\left(\underline{P}^{-\infty}\right)\right\}^{2}\right]
\end{aligned}
$$

for $r=2$. The foregoing two equations along with Eq. (B.1.11) may be used to formulate the first two moments of the Monte Carlo score, given the particle begins its flight to the left of the splitting surface.

Equation (B.1.15) may be simplified for $r=1$ and 2 and used in conjunction with Eq. (B.1.12) for particles with starting locations such that $z_{s}<z \leq D$ :

$$
\begin{aligned}
& \mathrm{Q}_{1,2}(\underline{\mathrm{P}})=\int_{\mathrm{z}_{\mathrm{s}} \mathrm{z}^{+}<\mathrm{D}} \mathrm{d \underline {P }}^{+} \mathrm{T}\left(\underline{\mathrm{P}}, \underline{\mathrm{P}}^{+}\right) \int \mathrm{d} \underline{\mathrm{P}}^{-} \sigma\left(\underline{\mathrm{P}}^{+}, \underline{\mathrm{P}}^{\prime}\right) \int \mathrm{d} s \mathrm{P}_{\mathrm{S}}\left(\underline{\mathrm{P}}^{-}, \mathrm{s}^{\prime} \mathrm{s}\right. \\
& +\int \underline{\mathrm{P}}^{+} \mathrm{D}_{\mathrm{T}}\left(\underline{\mathrm{P}}, \underline{\mathrm{P}}^{+}\right) \int \mathrm{ds} \mathrm{p}_{\mathrm{E}, \mathrm{D}}\left(\underline{\mathrm{P}}^{+}, \mathrm{s}\right) \mathrm{s}+\int \mathrm{dP} \underline{\mathrm{P}}^{+} \mathrm{T}\left(\underline{\mathrm{P}}, \underline{\mathrm{P}}^{+}\right) \mathrm{R}_{\mathrm{m}}\left(\underline{\mathrm{P}}^{+}\right) \int \mathrm{ds} \\
& \times \mathrm{p}_{\mathrm{R}}\left(\underline{\mathrm{P}}^{+}, \mathrm{s}\right) \mathrm{s}
\end{aligned}
$$

for $\mathbf{r}=1$, and 


$$
\begin{aligned}
& q_{2,2}(\underline{P})=\int_{z{ }_{s}<z^{+}<D} \underline{\mathrm{P}}^{+} \mathrm{T}\left(\underline{\mathrm{P}}, \underline{\mathrm{P}}^{+}\right) \int \underline{\mathrm{P}}^{\prime} \sigma\left(\underline{\mathrm{P}}^{+}, \underline{\mathrm{P}}^{\prime}\right) \int \mathrm{ds} \mathrm{P}_{\mathrm{S}}\left(\underline{\mathrm{P}}^{\prime}, \mathrm{s}\right) \int \underline{\mathrm{P}}^{-} \\
& \times\left[E\left(\underline{P}^{\prime}, \underline{P}^{\prime \prime}\right) \sum_{n=0}^{1}\left(\begin{array}{l}
2 \\
n
\end{array}\right) M_{n, 2}\left(\underline{P}^{\prime \prime}\right) s^{2-n}\right]+\int d \underline{P}^{+} D_{T}\left(\underline{P}, \underline{P}^{+}\right) \int d s
\end{aligned}
$$

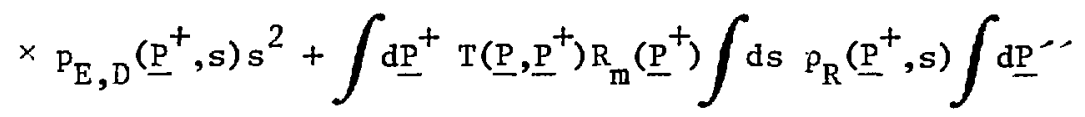

$$
\begin{aligned}
& \times B_{0 m}\left(\xi_{m} ; \underline{P}^{+}, P^{-\infty}\right) \quad \sum_{n=0}^{1}\left(\begin{array}{l}
2 \\
n
\end{array}\right) M_{n, z}\left(\underline{P}^{-\infty}\right) s^{2-n}
\end{aligned}
$$

for $r=2$.

Finally, for particles starting (or continuing) their flight from the splitting surface $\left(z=z_{s}\right)$, the following source terms are obtained from Eq. (B.1.16) for $\mathbf{r}=1$ and $\mathbf{r}=2$, respectively:

$$
\begin{aligned}
& \mathrm{Q}_{1, z} \underline{(\underline{\mathrm{P}})=} \int_{0<z^{+}<z_{s}} d \underline{\mathrm{P}}^{+} \mathrm{T}\left(\underline{\mathrm{P}}, \underline{\mathrm{P}}^{+}\right) \int \mathrm{dP}^{\prime} \sigma\left(\underline{\mathrm{P}}^{+}, \underline{\mathrm{P}}^{\prime}\right) \int \mathrm{ds} \mathrm{p}_{\mathrm{S}}\left(\underline{\mathrm{P}}^{\prime}, \mathrm{s}\right) \mathrm{s} \\
& +\int_{z_{s}<z^{+}<D} d \underline{P}^{+} T\left(\underline{P}, \underline{P}^{+}\right) \int d \underline{P}^{-} \sigma\left(\underline{P}^{+}, \underline{P}^{\prime}\right) \int d s p_{S}\left(\underline{P}^{\prime}, s\right) s+\int d \underline{P}^{+} \\
& \times D_{0}\left(\underline{P}, \underline{P}^{+}\right) \int d s p_{E, 0}\left(\underline{P}^{+}, s\right) s+\int d \underline{P}^{+} D_{T}\left(\underline{P}, \underline{P}^{+}\right) \int d s p_{E, D}\left(\underline{P}^{+}, s\right) s
\end{aligned}
$$

and 


$$
\begin{aligned}
& \mathrm{Q}_{2, z}(\underline{\mathrm{P}})=\int_{0<z^{+}<\mathrm{z}_{s}} \underline{\mathrm{dP}}^{+} \mathrm{T}\left(\underline{\mathrm{P}}, \underline{\mathrm{P}}^{+}\right) \int \underline{\mathrm{P}}^{-} \sigma\left(\underline{\mathrm{P}}^{+}, \underline{\mathrm{P}}^{\prime}\right) \int \mathrm{ds} \mathrm{p}_{\mathrm{S}}\left(\underline{\mathrm{P}}^{\prime}, \mathrm{s}\right) \int \underline{\mathrm{P}}^{-} \\
& \times\left[E\left(\underline{P}^{\prime}, \underline{P}^{\prime \prime}\right) \sum_{n=0}^{I}\left(\begin{array}{l}
2 \\
n
\end{array}\right) M_{n, I}\left(\underline{P}^{\prime \prime}\right) s^{2-n}\right]+\int_{z_{s}<z^{+}<D} d P^{+} T\left(\underline{p}, \underline{p}^{+}\right) \int \underline{d P}^{\prime} \\
& \times \sigma\left(\underline{\underline{P}}^{+}, \underline{\mathrm{p}}^{\prime}\right) \int \mathrm{ds} \mathrm{P}_{\mathrm{S}}\left(\underline{\mathrm{P}}^{\prime}, \mathrm{s}\right) \int \mathrm{d \underline {P } ^ { \prime }} E\left(\underline{\mathrm{P}}^{\prime}, \underline{\underline{P}}^{\prime \prime}\right) \sum_{n=0}^{1}\left(\begin{array}{l}
2 \\
n
\end{array}\right) M_{n, 2}\left(\mathrm{P}^{\prime \prime}\right) \mathrm{s}^{2-n} \\
& +\int \mathrm{dP}^{+} \mathrm{D}_{0}\left(\underline{\mathrm{P}}, \underline{\mathrm{P}}^{+}\right) \int \mathrm{ds} \mathrm{P}_{\mathrm{E}, 0}\left(\underline{\mathrm{P}}^{+}, \mathrm{s}\right) \mathrm{s}^{2}+\int \mathrm{dP}^{+} \mathrm{D}_{\mathrm{T}}\left(\underline{\mathrm{P}}, \underline{\mathrm{P}}^{+}\right) \int \mathrm{ds} \\
& \times P_{E, D}\left(\underline{P}^{+}, s\right) s^{2} \text {. }
\end{aligned}
$$

These equations are used in conjunction with Eq. (B.1.13).

The three sets of integral equations [Eqs. (B.1.11), (B.1.12), and (B.1.13), along with their respective source terms] may be further simplified by substituting the particular probability densities and kernels specific to the transport model assumed in this work. The app copriate forms for these kernels in terms of $z, \mu$, and coordinates ere presented in the beginning of Chapter IV. Also, the Monte Carlo tally in question is now formally specified to be the slab transmission probability. Hence, the only event at which non-zero scoring takes place is an escape past the back (tally) surface, $z=D$. When this occurs, the particle weight $w$ is scored with certainty. This is expressed mathematically by writing the appropriate expressions for the score distribution functions: 


$$
p_{E, 0}(\underline{P}, s)=p_{S}(\underline{P}, s)=p_{S m}(\underline{P}, s)=p_{R}(\underline{P}, s)=\delta(s),
$$

and

$$
\mathrm{P}_{E, \mathrm{D}}(\underline{\mathrm{P}}, \mathrm{s})=\delta(\mathrm{s}-\mathrm{w})
$$

The score distribution functions denoted above and the specific forms for $E\left(\underline{P}^{\prime}, \underline{P}^{\prime \prime}\right), \sigma\left(\underline{P}^{+}, \underline{P}^{\prime}\right), T\left(\underline{P}, \underline{P}^{\prime}\right), S_{m}\left(\underline{P}^{+}\right)$, and $B_{S m}\left(\underline{P}^{+}, \underline{P}^{\prime}\right)$ from the definitions at the beginning of Chapter IV are substituted in Eqs. (B.1.11), (B.1.20), and (B.1.21). The indicated integrations over the delta functions are then performed to yield the following equations expressing the first and second moments ( $r=1$ and 2$)$ of the transmission score, given the particle begins its flight with $z$, such that $0 \leq z<z_{s}$ :

$$
\begin{aligned}
& M_{r, 1}(z, \mu, w)=\int_{0<z^{+}<z_{s}} \mathrm{~d} z^{+} \int \mathrm{d} \mu^{\prime} \frac{\Sigma_{T}}{|\mu|} \mathrm{T}\left(z \rightarrow z^{+}, \mu\right) E\left(z^{+}, \mu \rightarrow \mu^{\prime \prime}\right) \\
& \quad \times M_{r, 1}\left(z^{+}, \mu^{\prime}, w_{s} w\right)+H(\mu) T\left(z \rightarrow z_{s}, \mu\right) \cdot m^{\prime} \cdot M_{r, z_{s}}\left(z_{s}, \mu, w / m\right) \\
& +Q_{r, 1}(z, \mu, w),
\end{aligned}
$$

with

$$
Q_{1,1}(z, \mu, w)=0 \quad \text { and }
$$

$$
Q_{2,1}(z, \mu, w)=H(\mu) \cdot m(m-1) \cdot T\left(z \rightarrow z_{s}, \mu\right)\left\{M_{1, z_{s}}\left(z_{s}, \mu, w / m\right)\right\}^{2} .
$$

Note that the restriction of starting locations to the lefi of the splitting surface implies that $T\left(z \rightarrow z_{s}, \mu\right)=0$ for starting directions $\mu<0$ (recall the definition of the transmission probability). For 
this reason (and also due to the Heaviside function on $\mu$ ), the second term in Eq. (B.1.26a) drops out for $\mu<0$, and $Q_{2,1}(z, \mu<0, w)=0$. Similar substitution in Eqs. (B.1.12), (B.1.22), and (B.1.23) and their integration yields the following moment equations (and source terms) for the case of particles starting at $z$, such that $z_{s}<z \leq D$ :

$$
\begin{aligned}
& M_{r, 2}(z, \mu, w)=\int d z^{+} \int d \mu^{-} \frac{\Sigma_{T}}{|\mu|} T\left(z \rightarrow z^{+}, \mu\right) E\left(z^{+}, \mu \rightarrow \mu^{-\prime}\right) \\
& z_{s}<z^{+}<D \\
& \times M_{r, 2}\left(z^{+}, \mu^{\prime \prime}, w_{s} w\right)+[1-H(\mu)] \cdot T\left(z \rightarrow z_{s}, \mu\right) \cdot \xi_{\text {m }} \\
& \times M_{r, z_{s}}\left(z_{s}, \mu, w / \xi_{m}\right)+Q_{r, 2}(z, \mu, w),
\end{aligned}
$$

where

$$
\mathrm{Q}_{1,2}(z, \mu, \mathrm{w})=\mathrm{H}(\mu) \cdot \mathrm{T}(z+\mathrm{D}, \mu) \cdot \mathrm{w}
$$

and

$$
Q_{2,2}(z, \mu, w)=H(\mu) \cdot T(z \rightarrow D, \mu) \cdot w^{2} .
$$

In writing Eq. (B.1.27a), the property stated in Eq. (4.1.1.6) was used to eliminate the term involving ${ }_{r, z_{S}}\left(z_{s}, \mu, 0\right)$. Again, the restriction of starting positions to the range $z_{s}<z \leq D$ (as well as the presence of the Heaviside function) implies that the second term of Eq. (B.1.27a) drops out for $\mu>0$ [note the definition of $T\left(z \rightarrow z_{s}, \mu\right)$ ]. For similar reasons, both $Q_{1,2}$ and $Q_{2,2}=0$ for $\mu<0$.

Finally, for particles starting (or resuming) their flight from the splitting surface $\left(z=z_{s}\right)$, the same procedure of substitution and integration applied to Eqs. (B.1.13), (B.1.24), and (B.1.25) ylelds 


$$
\begin{aligned}
& \mathrm{M}_{r, z_{s}}\left(z_{s}, \mu, w\right)=\int_{0<z^{+<z} s} \mathrm{~d} z^{+} \int \mathrm{d} \mu^{--} \frac{\Sigma_{\mathrm{T}}}{|\mu|} \mathrm{T}\left(\mathrm{z}_{\mathrm{s}} \rightarrow z^{+}, \mu\right) \mathrm{E}\left(\mathrm{z}^{+}, \mu \rightarrow \mu^{-\infty}\right) \\
& \times M_{r, I}\left(z^{+}, \mu^{-\infty}, w_{s} w\right)+\int_{z_{s}<z^{+}<D} d z^{+} \int d \mu^{-} \frac{\Sigma_{T}}{|\mu|} T\left(z_{s} \rightarrow z^{+}, \mu\right) \\
& \times E\left(z^{+}, \mu \rightarrow \mu^{\prime}\right) M_{r, 2}\left(z^{+}, \mu^{\prime}, w_{s} w\right)+Q_{r, z_{s}}\left(z_{s}, \mu, w\right), \\
& \text { with } \quad Q_{1, z_{s}}\left(z_{s}, \mu, w\right)=H(\mu) \cdot T\left(z_{s} \rightarrow D, \mu\right) \cdot w \\
& \text { and } \quad Q_{2, z_{s}}\left(z_{s}, \mu, w\right)=H(\mu) \cdot T\left(z_{s} \rightarrow D, \mu\right) \cdot w^{2} \text {. }
\end{aligned}
$$

Due to the definition of the transmission probability and the presence of the Heaviside function, for starting directions $\mu<0$, it is true that $Q_{1, z_{s}}=0, Q_{2, z_{s}}=0$, and the second term in Eq. (B.1.28a) vanishes. Similarly, the first term of the equation is eliminated for $\mu>0$.

Thus, the coupled integral equations [Eqs. (B.1.26), (B.1.27), and (B. 1.28) ] express the first two moments of the Monte Carlo transmission score over the entire range of starting positions $z$ :

$$
M_{r}(z, \mu, w)=\left\{\begin{array}{lll}
M_{r, 1}(z, \mu, w) & \text { for } & 0 \leq z<z_{s} \\
M_{r, 2}(z, \mu, w) & \text { for } & z_{s}<z \leq D, \\
M_{r, z_{s}}(z, \mu, w) & \text { for } z=z_{s}
\end{array}\right. \text { and }
$$




\section{B.2 Conditions at the Splitting Surface}

It is essential in this development to investigate the behavior of the solution functions, i.e., the first and second moments $\left(M_{1}\right.$ and $M_{2}$ ), at the internal splitting surface. The equations for $M_{r, 1}$ and $M_{r, 2}$ are coupled through $M_{r, z_{S}}$. Formally, the three cyuations may be reduced to two simply by substituting for $M_{r, z_{s}}$ in both Eqs. (B.1.26) and (B.1.27). However, before actually working further with these equations, it is important to be able to demo.strate the property of weight separability in the $r$-th moments. In other words, weightmodifying (muitiplying) factors may be brought outside the argument list of the $r$-th moment. The mathematical statement is made very generally here:

$$
M_{r}\left(z, \mu, w^{-} w\right)=w^{-1} M_{r}(z, \mu, w),
$$

where $w^{\prime}$ is any weight multiplier. The proof of this property for the Monte Car1o problem being considered in this work appears in Appendix D. Before proceeding with the derivations, it is helpful to define a new transition kerne1 as

$$
\mathrm{K}\left(z, \mu \rightarrow z^{+}, \mu^{-\prime}\right) \equiv \frac{\Sigma_{\mathrm{T}}}{|\mu|} \cdot \mathrm{T}\left(\mathrm{z} \rightarrow \mathrm{z}^{+}, \mu\right) \mathrm{E}\left(\mathrm{z}^{+}, \mu \rightarrow \mu^{\prime \prime}\right)
$$

Also, the following conditions follow from the definition of the transmission probability (see beginning of Chapter IV):

$$
T\left(z \rightarrow z_{s}, \mu\right) \cdot T\left(z_{s} \rightarrow z^{\prime}, \mu\right)=T\left(z \rightarrow z^{\prime}, \mu\right)
$$

and

$$
T\left(z \rightarrow z_{s}, \mu\right) \cdot T\left(z_{s} \rightarrow D, \mu\right)=T(z \rightarrow D, \mu)
$$


Now employing Eqs. (B.1.28), the appropriate expression for $\mathbf{M}_{\mathbf{r}, \mathbf{z}_{\mathbf{S}}}$ is substituted into Eqs. (B.1.26). By using the conditions stated in Eqs. (B.2.1), (B.2.2), and (B.2.3), as well as combining the source terms with the appropriate $r$-th moment equation, the formulations for $M_{1,1}$ and $M_{2,1}$ are written:

$$
\begin{aligned}
& M_{1,1}(z, \mu, w)=w_{s} \int_{0<z^{+}<z s} d^{+} \int d^{\cdots} k\left(z, \mu \rightarrow z^{+}, \mu^{-\cdots}\right) M_{1,1}\left(z^{+}, \mu^{\prime,}, w\right) \\
& +w_{s} \int d z^{+} \int d \mu^{-} k\left(z, \mu \rightarrow z^{+}, \mu^{-}\right) M_{1,2}\left(z^{+}, \mu^{-}, w\right) \\
& z_{s}<z^{+}<D
\end{aligned}
$$$$
+H(\mu) \cdot T(z \rightarrow D, \mu) \cdot w
$$

and

$$
\begin{aligned}
& M_{2,1}(z, \mu, w)=w_{s}^{2} \int_{0<z^{+}<z s} d z^{+} \int d \mu^{--} k\left(z, \mu \rightarrow z^{+}, \mu^{-\top}\right) M_{2,1}\left(z^{+}, \mu^{-\cdots}, w\right) \\
& +\frac{w_{s}^{2}}{m} \int_{z_{s}<z^{+}<D} d z^{+} \int d \mu^{-} k\left(z, \mu \rightarrow z^{+}, \mu^{-\cdots}\right) M_{2,2}\left(z^{+}, \mu^{-,}, w\right) \\
& +H(\mu) \cdot T(z \rightarrow D, \mu) \cdot \frac{w^{2}}{m}+H(\mu) \cdot\left(\frac{m-1}{m}\right) \cdot T\left(z \rightarrow z_{s}, \mu\right) \\
& \times\left\{M_{1, z_{s}}\left(z_{s}, \mu, w\right)\right\}^{2} .
\end{aligned}
$$

In performing the substitution, some terms in the expression for $\mathrm{M}_{\mathbf{r}, \mathbf{z}_{\mathbf{S}}}$ dropped out due to the restriction to positive directions $(\mu>0)$ imposed by the transmission probability, i.e., $T\left(z \rightarrow z_{s}, \mu\right)$ in 
Eq. (B.1.26a). Recall that starting locations $z$ are restricted in

Eq. (B.1.26a) to the range $0 \leq z<z_{s}$.

Similar use of Eqs. (B.1.28) in the substitution for $\mathrm{M}_{\mathbf{r}, z_{\mathbf{s}}}$ in

Eq. (B.1.27) yields the following formulations for $M_{1,2}$ and $M_{2,2}$ :

$$
\begin{aligned}
& M_{1,2}(z, \mu, w)=w_{S} \int d z^{+} \int d \mu^{-} k\left(z, \mu+z^{+}, \mu^{-\infty}\right) M_{1,2}\left(z^{+}, \mu^{-}, w\right) \\
& z_{s}<z^{+}<D \\
& +w_{s} \int_{0<z^{+}<z_{s}} d z^{+} \int \mathrm{d} \mu^{\cdots} \mathrm{k}\left(z, \mu \rightarrow z^{+}, \mu^{\cdots}\right) \mathrm{M}_{1,1}\left(z^{+}, \mu^{\cdots}, w\right) \\
& +H(\mu) \cdot T(z \rightarrow D, \mu) \cdot w
\end{aligned}
$$

and

$$
\begin{aligned}
& M_{2,2}(z, \mu, w)=w_{s}^{2} \int_{z_{s}<z^{+}<D} d z^{+} \int d^{-} K\left(z, \mu+z^{+}, \mu^{-}\right) M_{2,2}\left(z^{+}, \mu^{-,}, w\right) \\
& +\mathrm{m} \cdot \mathrm{w}_{\mathrm{s}}^{2} \int_{0<z^{+}<z s} \mathrm{~d} z^{+} \int \mathrm{d} \mu^{-} \mathrm{K}\left(\mathrm{z}, \mu \rightarrow \mathrm{z}^{+}, \mu^{-\prime}\right) \mathrm{M}_{2,1}\left(\mathrm{z}^{+}, \mu^{\prime}, w\right) \\
& +H(\mu) \cdot T(z \rightarrow D, \mu) \cdot w^{2} .
\end{aligned}
$$

Given Eqs. (B.2.4) and (B.2.5), the behavior of the moment functions as $z$ (starting location) approaches the splitting surface $z_{s}$ may now be ascextained. The following definitions are made:

$$
\begin{aligned}
& M_{r}^{-}\left(z_{s}, \mu, w\right) \lim _{z \rightarrow z_{s^{-}}} M_{r, 1}(z, \mu, w) \\
& \text { and } \quad M_{r}^{+}\left(z_{s}, \mu, w\right) \equiv \lim _{z \rightarrow z_{s+}} M_{r, 2}(z, \mu, w)
\end{aligned}
$$


On taking the limit of Eqs. (B.2.4a) and (B.2.5a) as the starting location is allowed tc approach the splitting surface from bi low and above, respectively:

$$
\begin{aligned}
& M_{1}{ }^{-}(z, \mu, w)=w_{s} \int_{0<z^{+}<z s} \mathrm{dz}^{+} \int \mathrm{d}^{\cdots} \mathrm{K}\left(z_{\mathrm{s}}, \mu \rightarrow \mathrm{z}^{+}, \mu^{-{ }^{+}}\right) \mathrm{M}_{1,1}\left(z^{+}, \mu^{-}, w\right) \\
& +w_{s} \int_{z_{s}<z^{+}<D} d z^{+} \int d \mu^{-} k\left(z_{s}, \mu \rightarrow z^{+}, \mu^{-\infty}\right) M_{1,2}\left(z^{+}, \mu^{-\cdots}, w^{+}\right) \\
& +H(\mu) \mathrm{T}\left(z_{s} \rightarrow \mathrm{D}, \mu\right) \cdot w
\end{aligned}
$$

and

$$
\begin{aligned}
& M_{1}^{+}\left(z_{s}, \mu, w\right)=w_{s} \int_{z_{s}<z^{+}<D} d z^{+} \int d \mu^{-\cdots} K\left(z_{s}, \mu \rightarrow z^{+}, \mu^{-\cdots}\right) M_{1,2}\left(z^{+}, \mu^{-\cdots}, w\right)
\end{aligned}
$$

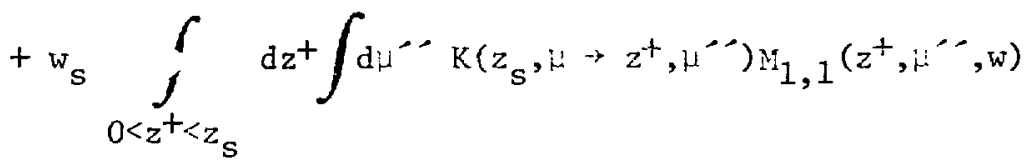

$$
\begin{aligned}
& +\mathrm{H}(\mu) \cdot \mathrm{T}\left(z_{\mathrm{s}} \rightarrow \mathrm{D}, \mu\right) \cdot \mathrm{w}
\end{aligned}
$$

Referring now to Equs. (B.2.7), (B.2.8), and (B.1.28a,b), it is evident that

$$
M_{1}{ }^{-}\left(z_{s}, \mu, w\right)=M_{1}^{+}\left(z_{s}, \mu, w\right)=M_{1, z_{s}}\left(z_{s}, \mu, w\right)
$$

The first moment solution is continuous across the splicting surface. Moreover, inspection of Eqs. (B.2.4a) and (B.2.5a) reveals that the integral equation governing the first moment is identical, no matter where the particle is introduced (or where it begins its flight). It 
is thus possible to write one integral equation for the first moment of the transmission score, applicable over the entire range of possible starting locations, i.e., $0 \leq z \leq D$ :

$$
\begin{gathered}
M_{1}(z, \mu, w)=w_{s} \int_{0<z^{+}<D} d z^{+} \int d \mu^{-\cdots} K\left(z, \mu+z^{+}, \mu^{-\infty}\right) M_{1}\left(z^{+}, \mu^{-}, w\right) \\
+H(\mu) \cdot T(z \rightarrow D, i) \cdot w
\end{gathered}
$$

We now consider the second moment. On taking the limit of Eq. (B.2.4b) as the starting location is allowed to approach $z_{s}$ from below:

$$
\begin{aligned}
& \mathrm{M}_{2}{ }^{-}\left(z_{s}, \mu, w\right)=w_{s}{ }^{2} \int_{0<z^{+}<z_{s}} d z^{+} \int \mathrm{d} \mu^{-} \mathrm{K}\left(z_{s}, \mu \rightarrow z^{+}, \mu^{-}\right) M_{2,1}\left(z^{+}, \mu^{-}, w\right) \\
& +\frac{w_{s}^{2}}{m} \int_{z_{s}<z^{+}<D} d z^{+} \int d \mu^{\cdots} \mathrm{K}\left(z_{s}, \mu+z^{+}, \mu^{\cdots}\right) M_{2,2}\left(z^{+}, \mu^{\cdots}, w\right) \\
& +H(\mu) \cdot T\left(z_{s} \rightarrow D, \mu\right) \cdot \frac{w^{2}}{m}+H(\mu) \cdot\left(\frac{m-1}{m}\right)\left\{M_{1, z_{s}}\left(z_{s}, \mu, w\right)\right\}^{2} .
\end{aligned}
$$

On taking the limit of Eq. (B.2.5b) as $z$ approaches $z_{s}$ from above:

$$
\begin{aligned}
& \left.\mathrm{M}_{2}^{+}\left(\mathrm{z}_{\mathrm{s}}, \mu, w\right)=\mathrm{w}_{\mathrm{s}}^{2} \int_{\mathrm{z}_{\mathrm{s}}<\mathrm{z}^{+}<\mathrm{D}} \mathrm{d} \mathrm{z}^{+} \int \mathrm{d} \mu^{-\infty} \mathrm{K}\left(\mathrm{z}_{\mathrm{s}}, \mu+\mathrm{z}^{+}, \mu^{-\cdots}\right) \mathrm{M}_{2,2} \mathrm{z}^{+}, \mu^{-}, w\right)
\end{aligned}
$$

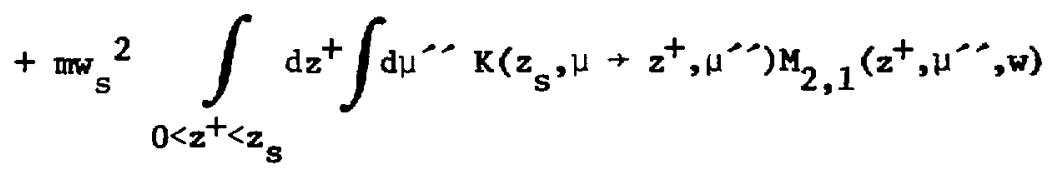

$$
\begin{aligned}
& +H(\mu) \cdot T\left(z_{s} \rightarrow D, \mu\right) \cdot w^{2} .
\end{aligned}
$$


Inspection of Eqs. (B.2.11), (B.2.12), and (B.1.28a,c) reveals that a discontinuity exists in the second moment solution at the splitting surface.

For $\underline{\mu}<\underline{0}$,

$$
M_{2}{ }^{-}\left(z_{s}, \mu, w\right)=\frac{1}{m} \cdot M_{2}{ }^{+}\left(z_{s}, \mu, w\right)=M_{2, z_{s}}\left(z_{s}, \mu, w\right),
$$

and for $\underline{\mu>0}$,

$$
\begin{aligned}
\mathrm{M}_{2}{ }^{+}\left(\mathrm{z}_{\mathrm{s}}, \mu, \mathrm{w}\right) & =\mathrm{m} \cdot \mathrm{M}_{2}^{-}\left(\mathrm{z}_{\mathrm{s}}, \mu, \mathrm{w}\right)-(\mathrm{m}-1)\left\{\mathrm{M}_{1, \mathrm{z}_{\mathrm{s}}}\left(\mathrm{z}_{\mathrm{s}}, \mu, \mathrm{w}\right)\right\}^{2} \\
& =\mathrm{M}_{2, \mathrm{z}_{\mathbf{s}}}\left(\mathrm{z}_{\mathrm{s}}, \mu, \mathrm{w}\right) .
\end{aligned}
$$

Thus, the linear $r-t h$ moment $(r=1,2)$ integral equations for the slab transmission score are formulated in Eqs. (B.1.26), (B.1.27), and (B.1.28), depending on where the particle is considered to begin its f1ight. It was shown that for $r=1$ (first moment), these equations collapse to one which holds for all starting locations in the slab. For $r=2$ (second moment), the discontinuity in the solution function across the splitting surface is characterized by Eqs. (B.2.13) and (B. 2.14). 


\section{APPENDIX C}

\section{DERIVATION OF THE INTEGRAL (FIRST MOLENT) EQUATION \\ FOR TIME PER PARTICLE HISTORY (SINGLE SFIITTING SURFACE)}

The development of the integral equations governing the time per particle history $\left[\tau_{p}(z, \mu)\right]$ is continued here from Eqs. (4.2.1.9a,b), $(4.2 .1 .10 a, b)$, and $(4 \cdot 2 \cdot 1.7 a, b)$. Following a statement of the equations in terms of $(z, \mu)$ coordinates, the discontinuity in the solution function at the splitting surface $\left(x_{x_{s}}\right)$ is characterized.

\section{C.1 The First Moment Integral Equations for $\tau_{p}$}

Unlike the case for the moments of the transmission score, when considering expected time per particle history a score may be considered to be made at each of the five possible events of the particle's history. Specifically, the score at an event is the actual computer time required to process the particular event. The score distribution functions for this situation may be written as

$$
\begin{aligned}
& P_{E, 0}\left(\underline{R}^{\prime}, s\right) d s=\delta\left(s-\tau_{0}\right) d s, \\
& \mathrm{p}_{E, D}\left(\underline{R}^{\prime}, s\right) \mathrm{ds}=\delta\left(s-\tau_{\mathrm{T}}\right) \mathrm{ds}, \\
& \mathrm{p}_{\mathrm{S}}\left(\underline{R}^{-}, s\right) \mathrm{d} s=\delta\left(s-\tau_{\mathrm{c}}\right) \mathrm{ds}, \\
& \operatorname{P}_{S m}\left(\underline{R}^{\prime}, s\right) d s=\delta\left(s-\tau_{s}\right) d s,
\end{aligned}
$$

and

$$
p_{R}\left(\underline{R}^{\circ}, s\right) d s=\delta\left(s-\tau_{R}\right) d s
$$

Here $\tau_{0}, \tau_{T}, \tau_{C}, \tau_{s}$, and $\tau_{R}$ represent the actual (measured) computer times required to process a front-surface escape, tally-surface escape, 
collision event, splitting event, and Russian roulette event, resr -ctively.

The score distribution functions as defined above as well as the specific forms of the probability deisities and kernels (see definitions in Sections 4.1.1 and 4.2.1) are substituted into Eqs. (4.2.1.9). The appropriate integrations over the de1ta functions are carried out and identity (4.1.1.12) is employed to yield the following equation for $\tau_{p}(z, \mu)$, given the particle's starting location is restricted to the left of the splitting surface $\left(0 \leq z<z_{s}\right)$ :

$$
\begin{aligned}
& \tau_{p, 1}(z, \mu)=\int_{0<z^{+}<z s} \mathrm{dz}^{+} \int \mathrm{d}^{\cdots} \mathrm{K}\left(z, \mu \rightarrow z^{+}, \mu^{-\prime}\right) \tau_{\mathrm{p}, 1}\left(z^{+}, \mu^{-\prime}\right) \\
& +H(\mu) \cdot m \cdot T\left(z \rightarrow z_{s}, \mu\right) \tau_{p, z_{s}}\left(z_{s}, \mu\right)+\int_{0<z^{+}<z_{s}} \mathrm{dz}{ }^{+\cdot \frac{\Sigma}{T}} \frac{|\mu|}{\mid \mu} \\
& x T\left(z \rightarrow z^{+}, \mu\right) \cdot \tau_{c}+[1-H(\mu)] \cdot \Gamma(z \rightarrow 0, \mu) \cdot \tau_{0} \\
& +H(\mu) T\left(z \rightarrow z_{s}, \mu\right) \cdot \tau_{s} .
\end{aligned}
$$

In writing Eq. (C.1.2) the source terms have been recombined with the main equation. Also note that the restriction of starting locations to $0 \leq z<z_{s}$ and the definition of the transmission probability $1 m p l y$ that the second and last terms of Eq. (C.1.2) vanish for directions $\mu<0$. The Heaviside functions in these terms are redundant, since the restriction to positive $\mu$ values is already implied by the transmission probabilities. Nevertheless, the Heaviside factors are retained for 
purposes of clartty. Similarly, the fourth term on the right-hand side of Eq. (C.1.2) vanishes for $\mu>0$.

After proper substitutions are made in Eqs. (4.2.1.7) for the score distributions and probability densities (as above), irtegrations over the delta functions are performed to give the following formulation for starting locations in the range $z_{S}<z \leq D$ :

$$
\begin{aligned}
& \tau_{p, 2}(z, \mu)=\int_{z_{\mathrm{S}}<z^{+}<\mathrm{D}} \mathrm{d} z^{+} \int \mathrm{d} \mu^{-} \mathrm{K}\left(7, \mu \rightarrow z^{+}, \mu^{\prime \prime}\right) \tau_{\mathrm{p}, 2^{-}}\left(z^{+}, \mu^{-\prime}\right) \\
& +[1-\mathrm{H}(\mu)] \cdot \frac{1}{\mathrm{~m}} \cdot \mathrm{T}\left(\mathrm{z} \rightarrow \mathrm{z}_{\mathrm{s}}, \mu\right) \mathrm{\tau}_{\mathrm{p}, \mathrm{z}_{\mathbf{s}}}\left(\mathrm{z}_{\mathrm{s}}, \mu\right)+\int_{\mathrm{z}_{\mathbf{s}}<\mathrm{z}^{+}<\mathrm{D}} \mathrm{d} \mathrm{z}^{+} \\
& \times \frac{\Sigma_{T}}{|\mu|} \mathrm{T}\left(z \rightarrow z^{+}, \mu\right) \cdot \tau_{c}+H(\mu) \mathrm{T}(z \rightarrow D, \mu) \cdot \tau_{T} \\
& +[1-H(\mu)] \mathrm{T}\left(z \rightarrow z_{s}, \mu\right) \cdot \tau_{R} .
\end{aligned}
$$

Again, due to the restrictions imposed by the transmission probabilities (and also by the Heaviside functions) several terms in the above equation vanish for either $\mu<0$ or $\mu>0$. Finally, Eqs. (4.2.1.10) for starting position $z=z_{s}$ are converted to 


$$
\begin{aligned}
& \tau_{p, z_{s}}(z, \mu)=\int_{0<z^{+}<z_{s}} \mathrm{~d} z^{+} \int \mathrm{d} \mu^{\cdots} \mathrm{K}\left(z_{s}, \mu \rightarrow z^{+}, \mu^{-\prime}\right) \tau_{p, 1}\left(z^{+}, \mu^{-\prime}\right)
\end{aligned}
$$

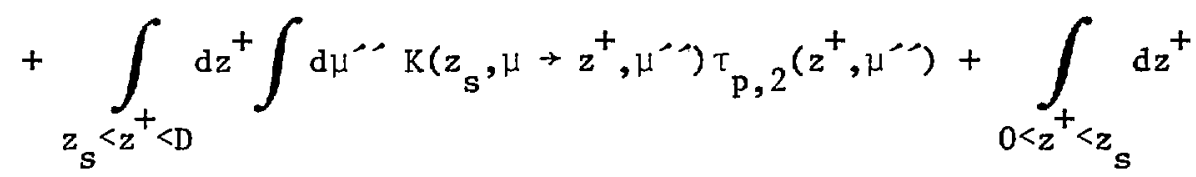

$$
\begin{aligned}
& \times \frac{\Sigma_{T}}{|\mu|} \mathrm{T}\left(z_{s} \rightarrow z^{+}, \mu\right) \cdot \tau_{c}+\int_{z_{S}<z^{+}<D} d z^{+} \frac{\Sigma_{T}}{|\mu|} T\left(z_{j} \rightarrow z^{+}, \mu\right) \cdot \tau_{c} \\
& +[1-H(\mu)] \mathrm{T}\left(z_{S} \rightarrow 0, \mu\right) \cdot \tau_{0}+H(\mu) \mathrm{T}\left(z_{S} \rightarrow D, \mu\right) \cdot \tau_{T} \text {. }
\end{aligned}
$$

Together, the three coupled integral equations [Eqs. (C.1.2), (C.1.3), and (c.1.4)] formulate the expected time per history over the entire range of starting positions in the slab.

\section{C.2 Discontinuity Condition at $z=z_{s}$.}

In order to characterize the discontinuity in the solution at $z=z_{s}$, the substitution for $\tau_{p, z_{s}}$ is made in both Eqs. (C.1.2) and (C.1.3).

Specifically, Eq. (C.1.4) is substituted into Eq. (C.1.2). Identities (4.1.1.12) and (B.2.3) are employed in order to express the equation for $\tau_{p, 1}\left(0 \leq z<z_{s}\right)$ as 


$$
\begin{aligned}
& \tau_{p, I}(z, \mu)=\int_{0<z^{+}<z_{s}} \mathrm{~d} z^{+} \int \mathrm{d} \mu^{\prime} \mathrm{k}\left(z, \mu \rightarrow z^{+}, \mu^{-\rho}\right) \tau_{p, 1}\left(z^{+}, \mu^{\prime \prime}\right)
\end{aligned}
$$

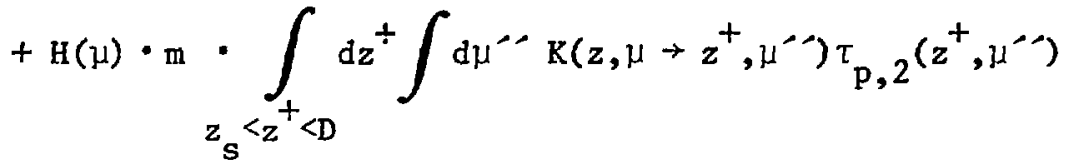

$$
\begin{aligned}
& +H(\mu) \cdot m \cdot \int_{z_{s}<z^{+}<D} d z+\frac{\Sigma_{T}}{|\mu|} T\left(z \rightarrow z^{+}, \mu\right) \cdot \tau_{c}+H(\mu) \cdot m \cdot T(z \rightarrow D, \mu) \cdot \tau_{T} \\
& +\int_{0<z^{+}<z_{s}} \mathrm{dz}+\frac{\Sigma_{\mathrm{T}}}{|\mu|} \mathrm{T}\left(\mathrm{z} \rightarrow \mathrm{z}^{+}, \mu\right) \cdot \tau_{c}+[1-\mathrm{H}(\mu)] \cdot \mathrm{T}(\mathrm{z} \rightarrow 0, \mu) \cdot \tau_{0} \\
& +\mathrm{H}(\mu) \mathrm{T}\left(z_{\rightarrow} \rightarrow z_{s}, \mu\right) \cdot \tau_{s} .
\end{aligned}
$$

It should be noted that the restriction to positive $\mu$ imposed by the Heaviside function in the second term (right-hand side) of Eq. (C.1.2) required that only terms in Eq. (C.1.4) which do not vanish for $\mu>0$ be admitted in the substitution for $\tau_{p, z_{s}}$.

Similarly, the identity

$$
T\left(z \rightarrow z_{s}, \mu\right) \cdot T\left(z_{s} \rightarrow 0, \mu\right)=T(z \rightarrow 0, \mu)
$$

along with identities (4.1.1.12) and (B.2.3) are employed in substituting Eq. (C.1.4) into Eq. (C.I.3). The resulting equation for $\tau_{p, 2}$ $\left(z_{s}<z \leq D\right)$ is 


$$
\begin{aligned}
& \tau_{p, 2}(z, \mu)=\int_{z_{s}<z^{+}<D} d z^{+} \int \mathrm{d} \mu^{-\mu} \mathrm{K}\left(z, \mu+z^{+}, \mu^{-\prime}\right) \tau_{p, 2}\left(z^{+}, \mu^{-\prime}\right)+[1-\mathrm{H}(\mu)] \\
& x \frac{1}{m} \cdot \int_{0<z^{+}<z s} \mathrm{dz}^{+} \int \mathrm{d} \mu^{-\infty} \mathrm{K}\left(\mathrm{z}, \mu \rightarrow \mathrm{z}^{+}, \mu^{-\rho}\right) \tau_{\mathrm{p}, 1}\left(\mathrm{z}^{+}, \mu^{-\rho}\right)+[1-\mathrm{H}(\mu)] \\
& \times \frac{1}{\mathrm{~m}} \cdot \int_{0<z^{+}<z \mathrm{~s}} \mathrm{dz}+\frac{\Sigma_{\mathrm{T}}}{|\mu|} \mathrm{T}\left(\mathrm{z} \rightarrow \mathrm{z}^{+}, \mu\right) \cdot \tau_{\mathrm{c}}+[1-\mathrm{H}(\mu)] \cdot \frac{1}{\mathrm{~m}} \cdot \mathrm{T}(\mathrm{z} \rightarrow 0, \mu) \cdot \tau_{C} \\
& +\int_{z_{S}<z^{+}<D} \mathrm{dz}+\frac{\Sigma_{T}}{|\mu|} \mathrm{T}\left(z \rightarrow z^{+}, \mu\right) \cdot \tau_{c}+H(\mu) T(z \rightarrow D, \mu) \cdot \tau_{T} \\
& +[1-H(\mu)] \mathrm{T}\left(z \rightarrow z_{s}, \mu\right) \cdot \tau_{R} .
\end{aligned}
$$

Again, only terms in Eq. (C.1.4) which do not vanish for $\mu<0$ are included in the substitution, due to the restriction imposed by the Heaviside function in the second term (right-hand side) of Eq. (C.1.3). The limit of Eq. (C.2.1) is now taken as the starting location $z$ is allowed to approach $z_{s}$ from below. With the definition

$$
\tau_{p}{ }^{-}\left(z_{s}, \mu\right) \equiv \lim _{z \rightarrow z_{s-}} \tau_{p, 1}(z, \mu),
$$

Eq. (C.2.1) becomes 


$$
\begin{aligned}
& \tau_{\mathrm{p}}^{-}\left(z_{\mathrm{s}}, \mu\right)=\int_{0<z^{+}<z_{s}} \mathrm{dz}^{+} \int \mathrm{d} \mu^{\prime} \mathrm{k}\left(z_{\mathrm{s}}, \mu \rightarrow z^{+}, \mu^{\prime \rho}\right) \tau_{\mathrm{p}, 1^{\prime}}\left(z^{+}, \mu^{-\rho}\right) \\
& +\mathrm{H}(\mu) \cdot \mathrm{m} \cdot \int_{z_{\mathrm{s}}<z^{+}<\mathrm{D}} \mathrm{dz} z^{+} \int \mathrm{d} \mu^{\prime \prime} \mathrm{K}\left(z_{\mathrm{s}}, \mu \rightarrow \mathrm{z}^{+}, \mu^{-\prime}\right) \tau_{\mathrm{p}, 2^{2}}\left(\mathrm{z}^{+}, \mu^{\prime \prime}\right) \\
& +\mathrm{H}(\mu) \cdot \mathrm{m} \cdot \int_{z_{S}<z^{+}<\nu} \mathrm{dz}^{+} \frac{\Sigma_{\mathrm{T}}}{|\mu|} \mathrm{T}\left(\mathrm{z}_{\mathrm{S}} \rightarrow \mathrm{z}^{+}, \mu\right) \cdot \tau_{\mathrm{c}}+\mathrm{H}(\mu) \cdot \mathrm{m} \cdot \mathrm{T}\left(\mathrm{z}_{\mathrm{s}} \rightarrow \mathrm{D}, \mu\right) \\
& \times \tau_{T}+\int_{0<z^{+}<z_{S}} d z^{+} \frac{\Sigma_{T}}{|\mu|} T\left(z_{S} \rightarrow z^{+}, \mu\right) \cdot \tau_{c}+[1-H(\mu)] \cdot T\left(z_{S} \rightarrow 0, \mu\right) \\
& \times \tau_{0}+H(\mu) \tau_{s} \text {. }
\end{aligned}
$$

Also, by defining

$$
\tau_{p}^{+}\left(z_{s}, \mu\right) \equiv \lim _{z \rightarrow z_{s+}} \tau_{p, 2}(z, \mu),
$$

the limit of Eq. (C.2.3) is taken as $z$ is allowed to approach $z_{s}$ from above: 


$$
\begin{aligned}
& \tau_{p}^{+}\left(z_{s}, \mu\right)=\int_{z_{s}<z^{+}<D} d z^{+} \int d \mu^{-\infty} k\left(z_{s}, \mu \rightarrow z^{+}, \mu^{-\infty}\right) \tau_{p, 2}\left(z^{+}, \mu^{-\infty}\right) \\
& +[1-H(\mu)] \cdot \frac{1}{m} \cdot \int_{C_{<z^{+}<z_{s}}} \mathrm{dz}^{+} \int \mathrm{d} \mu^{-\infty} \mathrm{K}\left(\mathrm{z}_{\mathrm{s}}, \mu \rightarrow \mathrm{z}^{+}, \mu^{-\infty}\right) \tau_{\mathrm{p}, 1}\left(\mathrm{z}^{+}, \mu^{-\infty}\right) \\
& +[1-H(\mu)] \cdot \frac{1}{m} \cdot \int_{0<z^{+}<z_{s}} d z^{+} \frac{\Sigma_{T}}{|\mu|} T\left(z_{s} \rightarrow z^{+}, \mu\right) \cdot \tau_{c}+[1-H(\mu)] \cdot \frac{1}{m} \\
& \times \mathrm{T}\left(z_{\mathrm{s}} \rightarrow 0, \mu\right) \cdot \tau_{0}+\int_{z_{\mathrm{s}}<z^{+}<\mathrm{D}} \mathrm{dz}+\frac{\Sigma_{\mathrm{T}}}{|\mu|} \mathrm{T}\left(\mathrm{z}_{\mathrm{s}} \rightarrow \mathrm{z}^{+}, \mu\right) \cdot \tau_{\mathrm{c}} \\
& +H(\mu) T\left(z_{s} \rightarrow D, \mu\right) \cdot \tau_{T}+[I-H(\mu)] \tau_{R} .
\end{aligned}
$$

The discontinuity in the solution function at $z=z_{s}$ is obtained after inspection of Eqs. (C.2.4b) and (C.2.5b). Thus, for $\mu<0$ :

$$
\tau_{p}^{+}\left(z_{s}, \mu\right)=\frac{1}{m} \cdot \tau_{p}^{-}\left(z_{s}, \mu\right)+\tau_{R},
$$

and for $\mu>0$ :

$$
\tau_{p}^{-}\left(z_{s}, i\right)=m \cdot \tau_{p}^{+}\left(z_{s}, \mu\right)+\tau_{s}
$$




\section{APPENDIX D}

WEIGHT SEPARABILITY IN THE $r$-th MOMENTS OF THE SLAB TRANSMISSTON SCORE

In order to facilitate the eventual numerical solution of the moment equations for the Monte Carlo slab transmission score, it is important to demonstrate the property of weight separability in the moments. In other words, we wish to show that

$$
M_{r}\left(\underline{R}, w^{-} w\right)=w^{-} M_{r}(\underline{R}, w),
$$

where $w^{\prime}$ is a weight-multiplying factor (constant with respect to $w$ ).

First, the basic definition of the $\mathrm{r}$-th moment of a Monte Carlo score (over the total score accumulation probability) is restated from Eq. $(4 \cdot 1.1 .5)$ as

$$
\mathrm{M}_{r}(\underline{R}, w) \equiv \int \psi(\underline{R}, w, s) s^{r} d s .
$$

The Monte Carlo problem being considered in this work simulates a true Markov process, i.e., the probability densities being sampled are not in any way affected by the past history of the particle. In other words, the particle weight (which may be regarded as a reflection of its past history) has no effect upon the random walk of the particle--probability densities are sampled irrespective of weight. Also, we are considering the case in which all event scores (tallies) are proportional to the particle weight. Thus, the probability that a particle starting out at $\mathrm{R}$ with weight $w^{-}$will eventually result in a total score s in ds is equal to the probability that a particle starting at $\underline{R}$, but with welght $w$, will score $s / w^{\prime}$ in $d\left(s / w^{\prime}\right)$. Therefore, 


$$
\psi\left(\underline{R}, w^{\prime} w, s\right) d s=\psi\left(\underline{R}, w, s / w^{\prime}\right) d\left(s / w^{\prime}\right) \quad .
$$

We can write the appropriate form of definition (D.2) for $M_{r}\left(\underline{R} . w^{\prime} w\right)$ as

$$
\begin{aligned}
M_{r}\left(\underline{R}, w^{\prime} w\right) & \equiv \int \psi\left(\underline{R}, w^{\prime} w, s\right) s^{r} d s \\
& =w^{-r} \int \psi\left(\underline{R}, w^{\prime} w, s\right)\left(s / w^{\prime}\right)^{r} d s,
\end{aligned}
$$

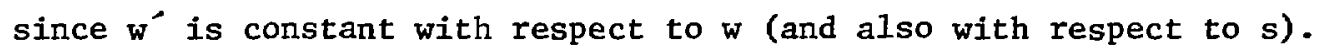
Substituting Eq. (D.3) into Eq. (D.4) and noting the definition of $M_{r}$ :

$$
M_{r}\left(\underline{R}, w^{\prime} w\right)=w^{-r} \int \psi\left(\underline{R}, w, s / w^{\prime}\right)\left(s / w^{\prime}\right)^{r} d\left(s / w^{\prime}\right),
$$

or $M_{r}\left(\underline{R}, w^{\prime} w\right)=w^{-r} M_{r}(\underline{D}, w)$. 
The purpose of this Appendix is to derive the identity stated as Eq. (4.1.2.1), namely

$$
\mathbf{R}_{z}^{+} C\left(z \rightarrow z^{\prime}, \mu\right)=\delta\left(z-z^{\prime}\right)
$$

where $\mathrm{R}_{\mathrm{z}}^{+}$represents the adjoint differential transport operator for the one-speed transport problem in one-dimensional slab geometry,

$$
\mathrm{R}_{\mathrm{z}}^{+}=\left\{-\frac{1}{\Sigma_{\mathrm{T}}} \mu \frac{\partial}{\partial z}+1\right\}
$$

Also, $C\left(z \rightarrow z^{-}, \mu\right)$ is the transport kernel, i.e., the probability that a particle starting out at $\mathrm{z}$ with direction $\mu$ will collide in $\mathrm{dz}^{\prime}$ about $\mathrm{z}^{\prime}$ before undergoing any other events:

$$
C\left(z+z^{\prime}, \mu\right) \mathrm{d} z^{\prime}=\frac{\Sigma_{\mathrm{T}}}{|\mu|} \cdot \mathrm{T}\left(z \rightarrow z^{\prime}, \mu\right) \mathrm{d} z^{\prime}
$$

From the definition stated at the beginning of Chapter IV, the transmission probability may also be written as follows:

$$
T\left(z \rightarrow z^{\prime}, \mu\right)= \begin{cases}\exp \left[-\Sigma_{T}\left(\frac{z^{-}-z}{\mu}\right)\right] H\left(z^{-}-z\right) & \text { for } \mu>0, \\ \exp \left[-\Sigma_{T}\left(\frac{z^{-}-z}{\mu}\right)\right] H\left(z-z^{\prime}\right) & \text { for } \mu<0, \text { (E.4) }\end{cases}
$$

The partial dérivative of $\mathrm{C}\left(\mathrm{z} \rightarrow \mathrm{z}^{\wedge}, \mu\right)$ with respect to $\mathrm{z}$ may be taken by considering Eqs. (E.3) and (E.4). 
For $\mu>0$,

$$
\begin{aligned}
& \frac{\partial}{\partial z} C\left(z \rightarrow z^{\prime}, \mu\right)=\frac{\Sigma_{T}}{\mu} \frac{\partial}{\partial z} T\left(z \rightarrow z^{\prime}, \mu\right) \\
& \quad=\frac{\Sigma_{T}}{\mu} H\left(z^{-}-z\right) \exp \left[-\Sigma_{T}\left(\frac{z^{\prime}-z}{\mu}\right)\right]\left(\frac{\Sigma_{T}}{\mu}\right) \\
& +\frac{\Sigma_{T}}{\mu} \exp \left[-\Sigma_{T}\left(\frac{z^{-}-z}{\mu}\right)\right] \delta\left(z^{-}-z\right)(-1)
\end{aligned}
$$

(Recall that the derivative of a Heaviside function is the Dirac delta function.)

Therefore $(\mu>0)$,

$$
\begin{aligned}
R_{z}^{+} C\left(z \rightarrow z^{-}, \mu\right) \equiv-\frac{\mu}{\Sigma_{T}} \frac{\partial}{\partial z} C\left(z \rightarrow z^{\prime}, \mu\right)+C\left(z \rightarrow z^{\prime}, \mu\right) \\
\quad=\exp \left[-\Sigma_{T}\left(\frac{z^{\prime}-z}{\mu}\right)\right] \delta\left(z^{\prime}-z\right)
\end{aligned}
$$

However, at $z^{-}=z$ the exponential factor in Eq. (E.6) is equal to one. Thus, for $\mu>0$

$$
R_{z}^{+} C\left(z \rightarrow z^{-}, \mu\right)=\delta\left(z^{-}-z\right)=\delta\left(z-z^{\prime}\right)
$$

Similarly, for $\underline{\mu<0}$

$$
\begin{aligned}
& \frac{\partial}{\partial z} C\left(z \rightarrow z^{\prime}, \mu\right)=\frac{\Sigma_{T}}{-\mu} H\left(z-z^{\prime}\right) \exp \left[-\Sigma_{T}\left(\frac{z^{\prime}-z}{\mu}\right)\right]\left(\frac{\Sigma_{T}}{\mu}\right) \\
& +\frac{\Sigma_{T}}{-u} \exp \left[-\Sigma_{T}\left(\frac{z^{-}-z}{\mu}\right)\right] \delta\left(z-z^{\prime}\right), \text { or } \\
& \mathrm{R}_{z}^{+} C\left(z \rightarrow z^{\prime}, \mu\right)=\exp \left[-\Sigma_{\mathrm{T}}\left(\frac{z^{-}-z}{\mu}\right)\right] \delta\left(z-z^{\prime}\right) \text { for } \mu<0 .
\end{aligned}
$$


Again (since the exponential term evaluates to unity when $z^{-}=z$ ), for $\underline{\mu<0}$

$$
\mathrm{R}_{z}^{+} \mathrm{C}\left(\mathrm{z} \rightarrow \mathrm{z}^{\prime}, \mu\right)=\delta\left(z-z^{\prime}\right)=\delta\left(z^{\prime}-z\right)
$$

Thus, from Eqs. (E.7) and (E.10), we see that for the general case (all $\mu \neq 0$ ),

$$
\mathrm{R}_{z}^{+} \mathrm{C}\left(z \rightarrow \mathrm{z}^{\prime}, \mu\right)=\delta\left(z-\mathrm{z}^{\prime}\right)
$$

By Eq. (E.3) and since $\frac{|\mu|}{\Sigma_{T}}$ is constant with respect to $R_{z^{+}}^{+}$it is also evident that

$$
\mathrm{R}_{\mathrm{z}}^{+} \mathrm{T}\left(\mathrm{z} \rightarrow \mathrm{z}^{-}, \mu\right)=\frac{|\mu|}{\Sigma_{\mathrm{T}}} \delta\left(z-\mathrm{z}^{\prime}\right) .
$$

Both identities (E.11) and (E.12) are employed in converting the integral moment equations to an integro-differential form (Sec. 4.1.2).

For reference, identity (E.11) is derived by Amster and Djonehri (c.f. Reference 14) for the generalized transport problem. 
APPENDIX F

FORTRAN Code Listing: MCS I 


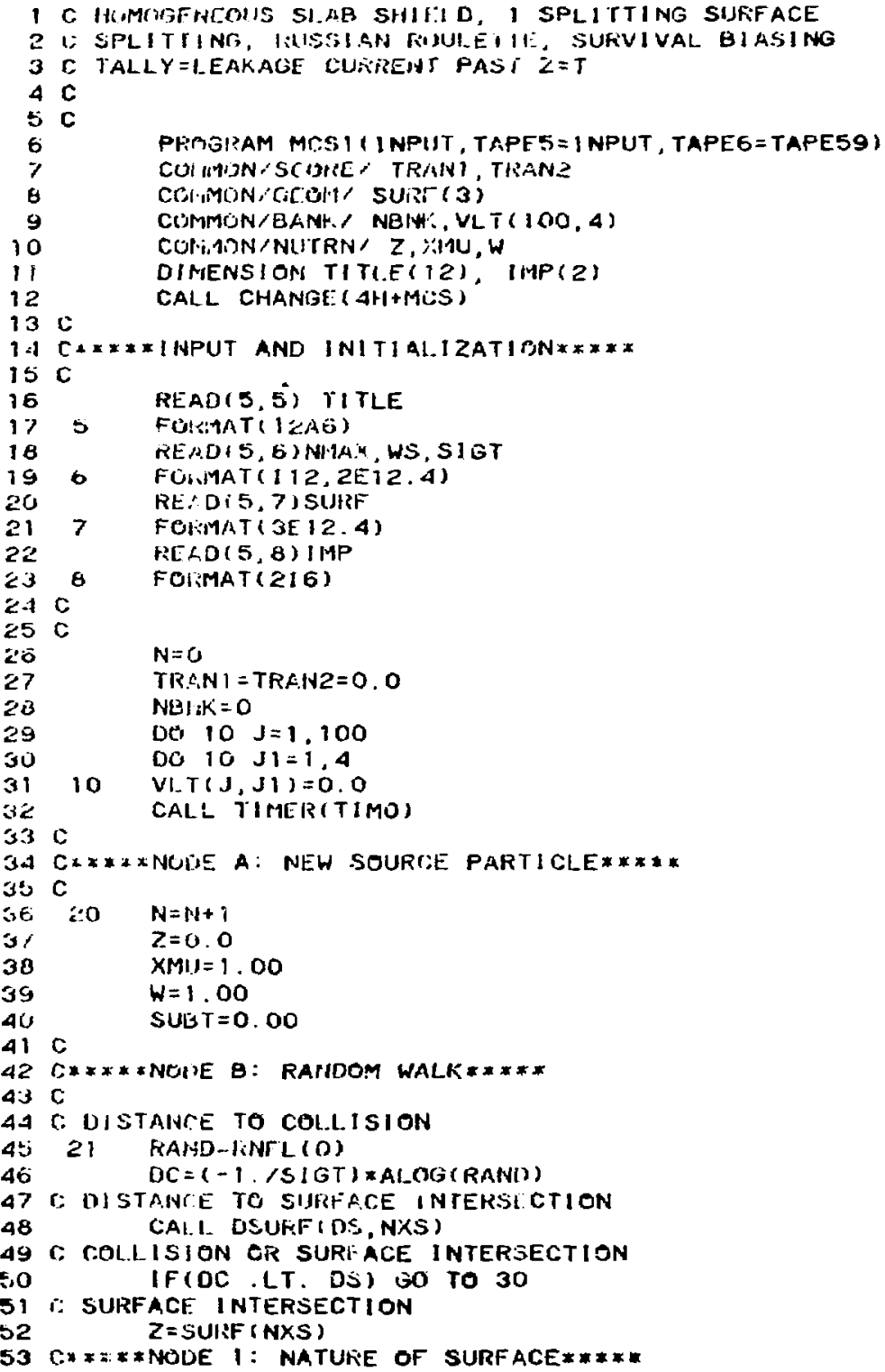




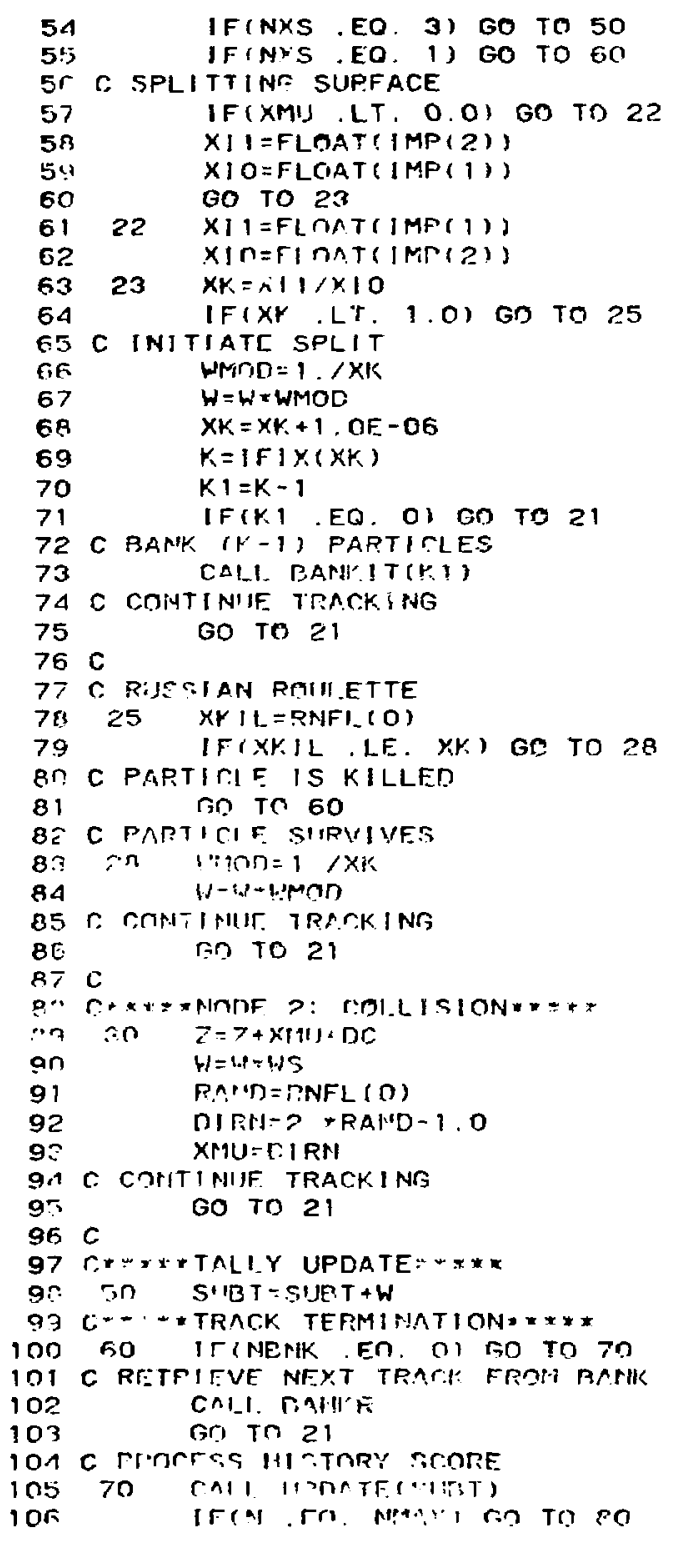



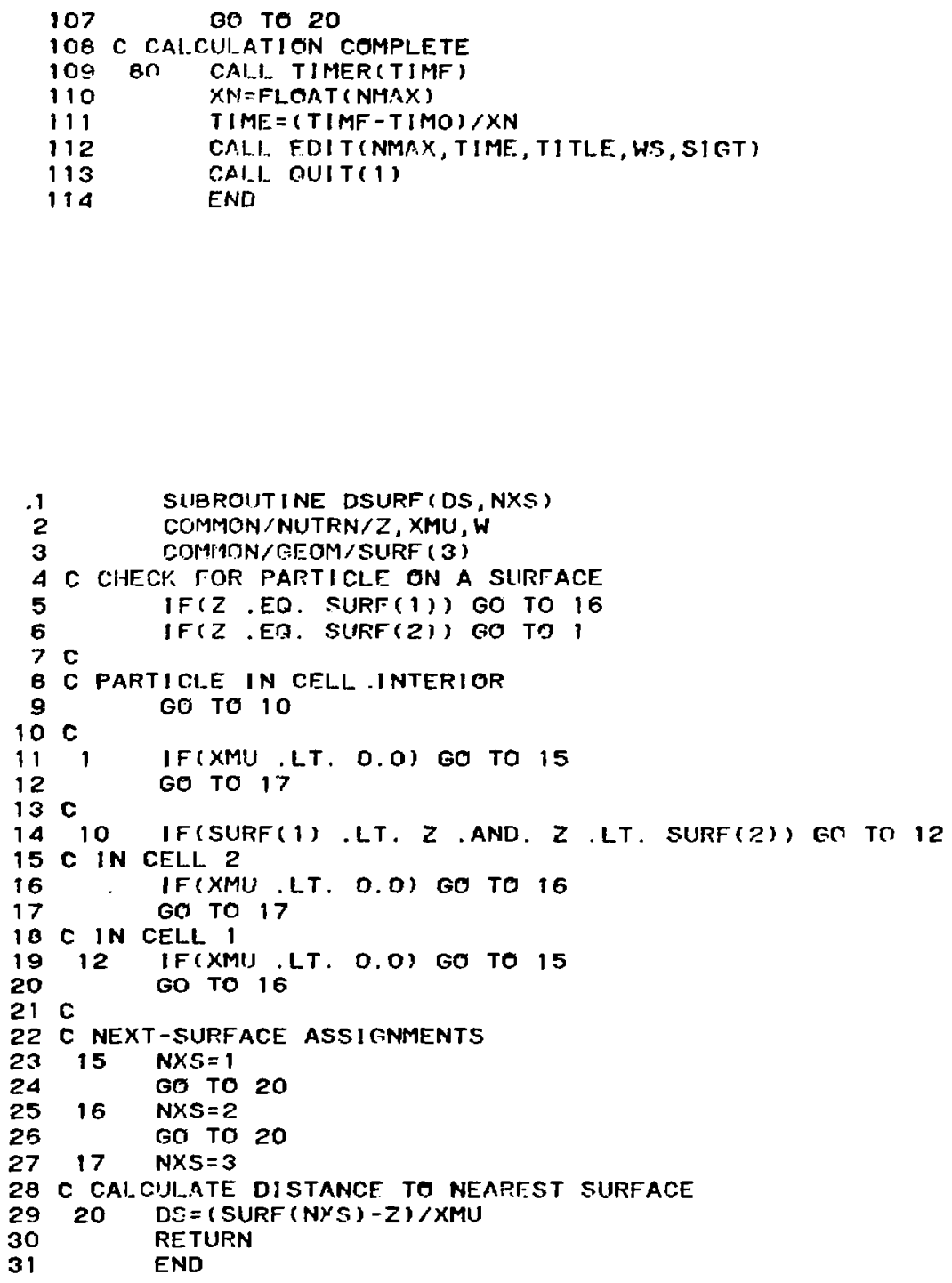


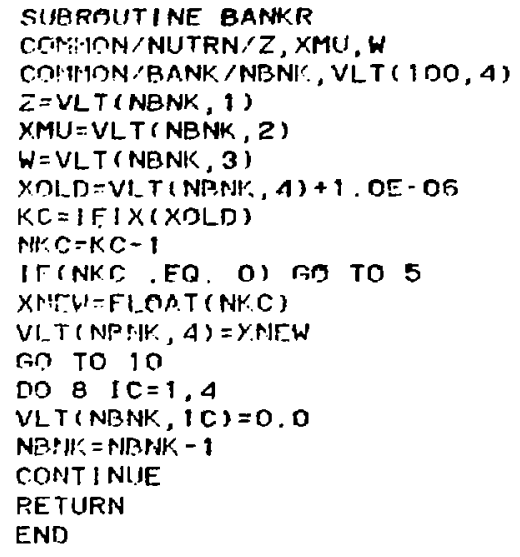




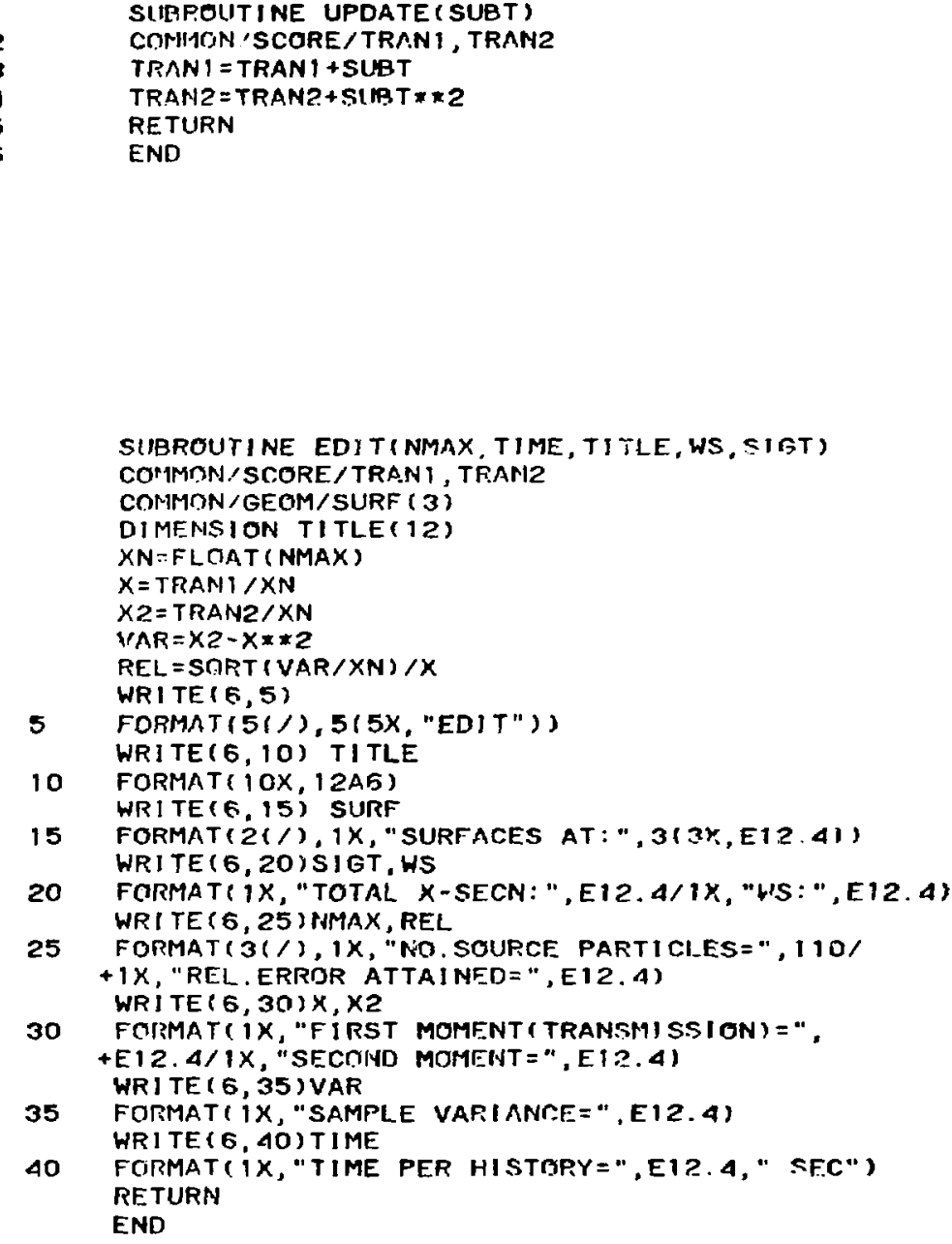

SUBROUTINE EDIT(NMAX, TIME, TITLE, WS, SIST) COMAMNISCORE/TRAN1, TRAN2

COMMON/GEOM/SURF (3)

DIMENISION TITLE (12)

$X N=F L C A T$ (NMAX)

$X=$ TRANI $/ X N$

$X 2=T R A N 2 / X N$

$M A R=X 2-X \times 2$

REL $=\operatorname{SART}(V A R / X N) / X$

WRI TE ( 6,5 )

5 FORMAT $(5(1), 5(5 X$, "EDIT"))

WRI TE $(6,10)$ TITLE

10 FORMAT (1OX, 12A5)

WRI TE $(6,15)$ SURF

15 FORMAT $(2 t /), 1 x$, "SURFACES AT:",3(3K, E12,41) WRITE $(6,20)$ SIGT, WS

20 FORMAT(1X, "TOTAL X-SECN: ", E12.4/1X, "WS:", E12.4) WRITE $(6,25$ I IMMAX, REL

25 FORMAT $(3(/), 1 X$, "NO. SOURCF PARTICIES =", $110 /$

$+1 X, " R E L$. ERRDR ATTAINED $=", E 12.4)$

WRI TE $(5,30) \times, \times 2$

30 FOIBMAT(IX, "FIRST MOMFNT (TRAMSMISSION)=", +E $12.4 / 1 X$, "SECTIYD MOMENT = ", E†?.4)

WRI TE ( 6,35 ) VAR

35 FORMAT $(1 X$, , SAMPLE VARIANCE =", E12.4)

HRI TE $(6,40)$ TIME

40 FCIRMAT(IX, "TIME PER HISTSTRY=", E12.4," SFC") RETURN

END

1

2

3

4

5

6

7

8

9

10

11

12

13

14

15

16

17

18

19

20

21

22

23

24

25

26

27

28

29 
APPENDIX G

FORTRAN Code Listing:

COSTFT 


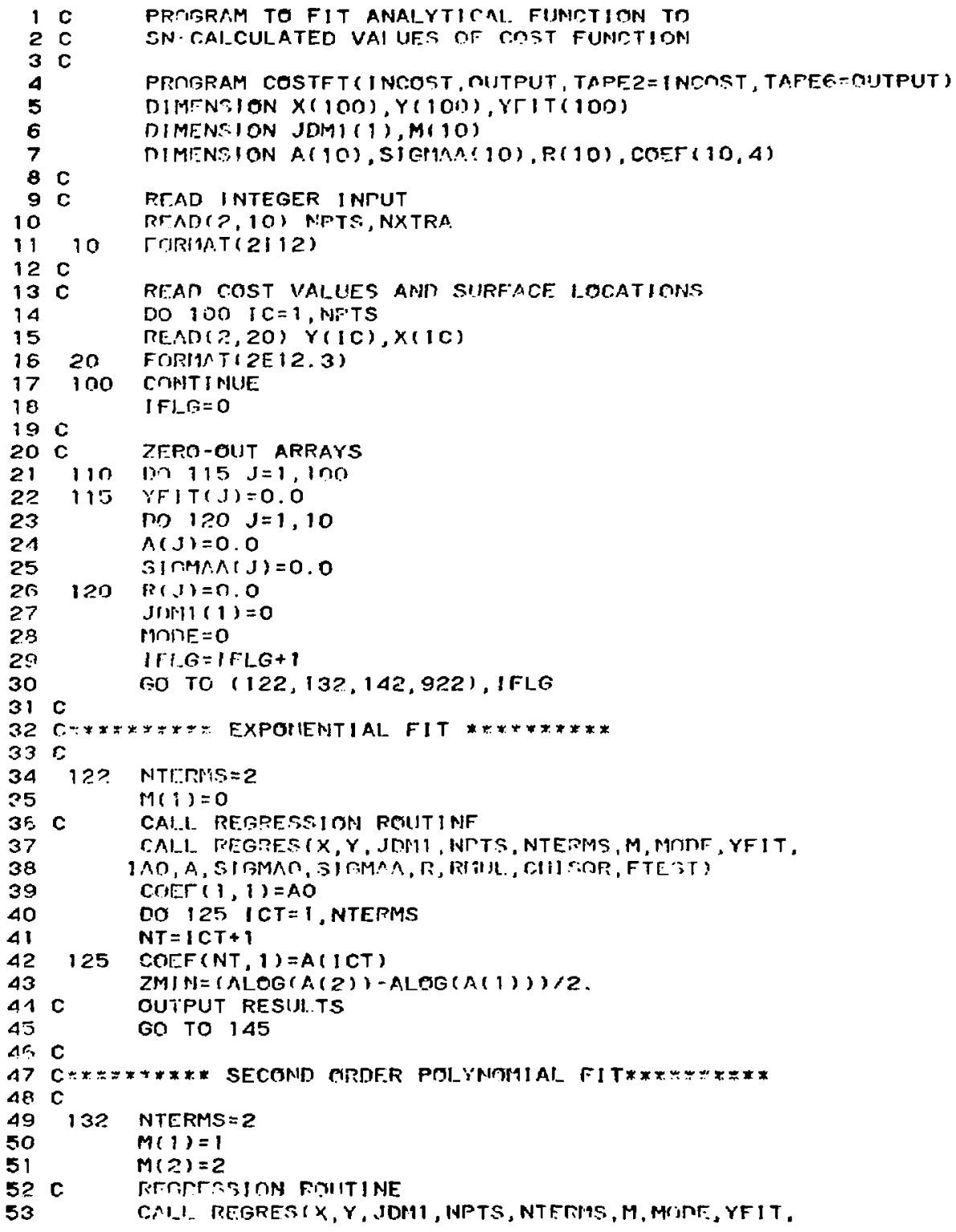




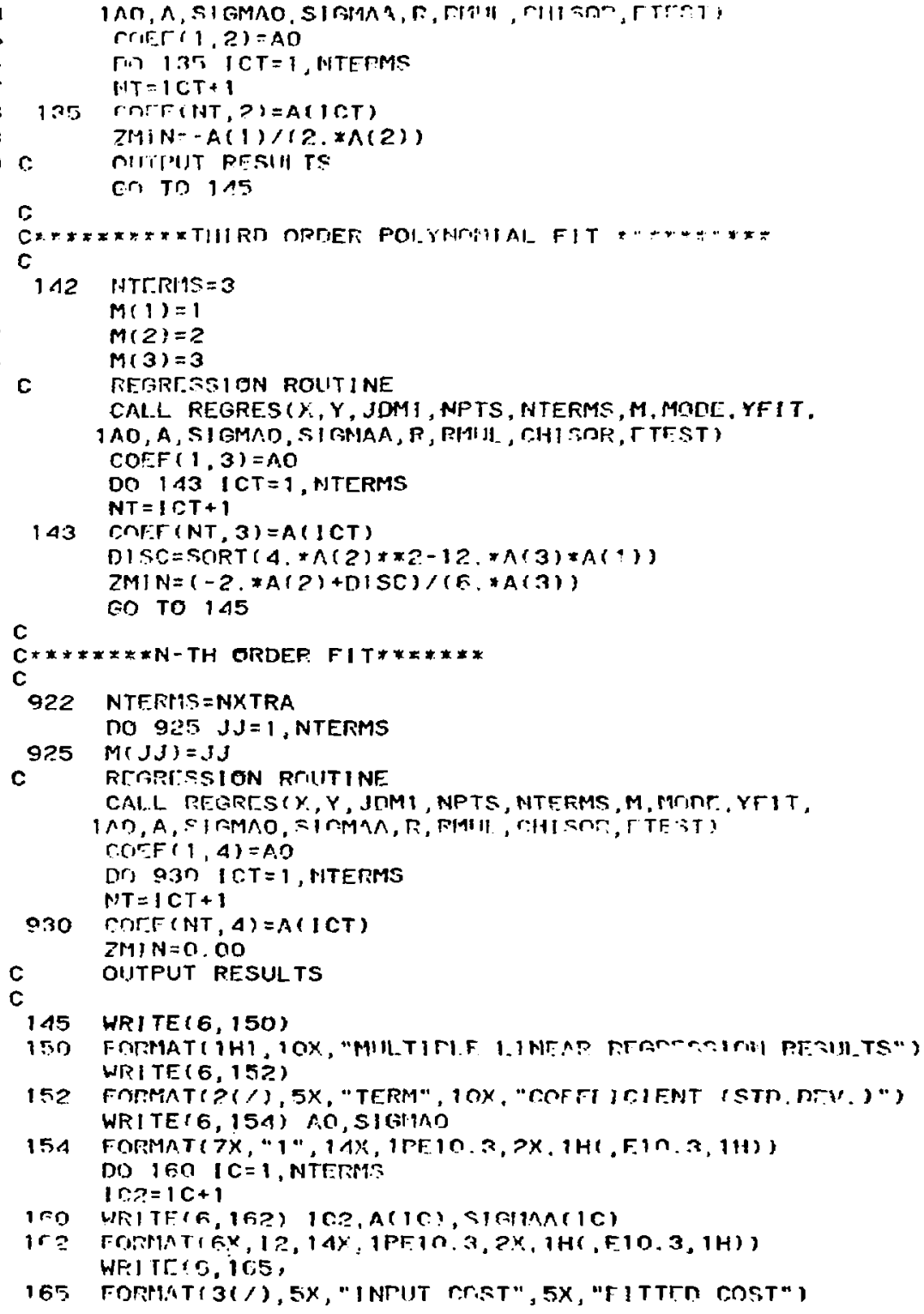




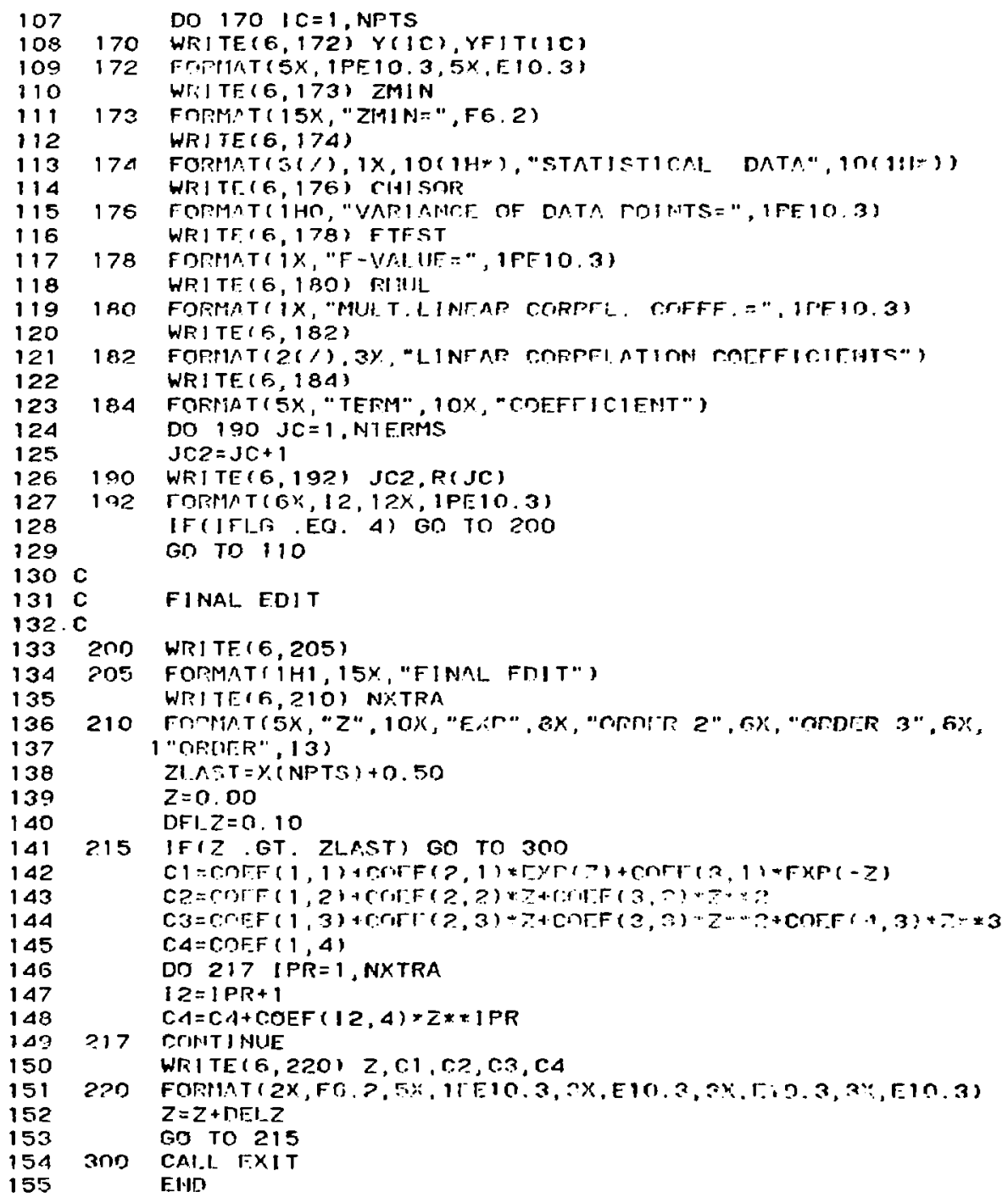




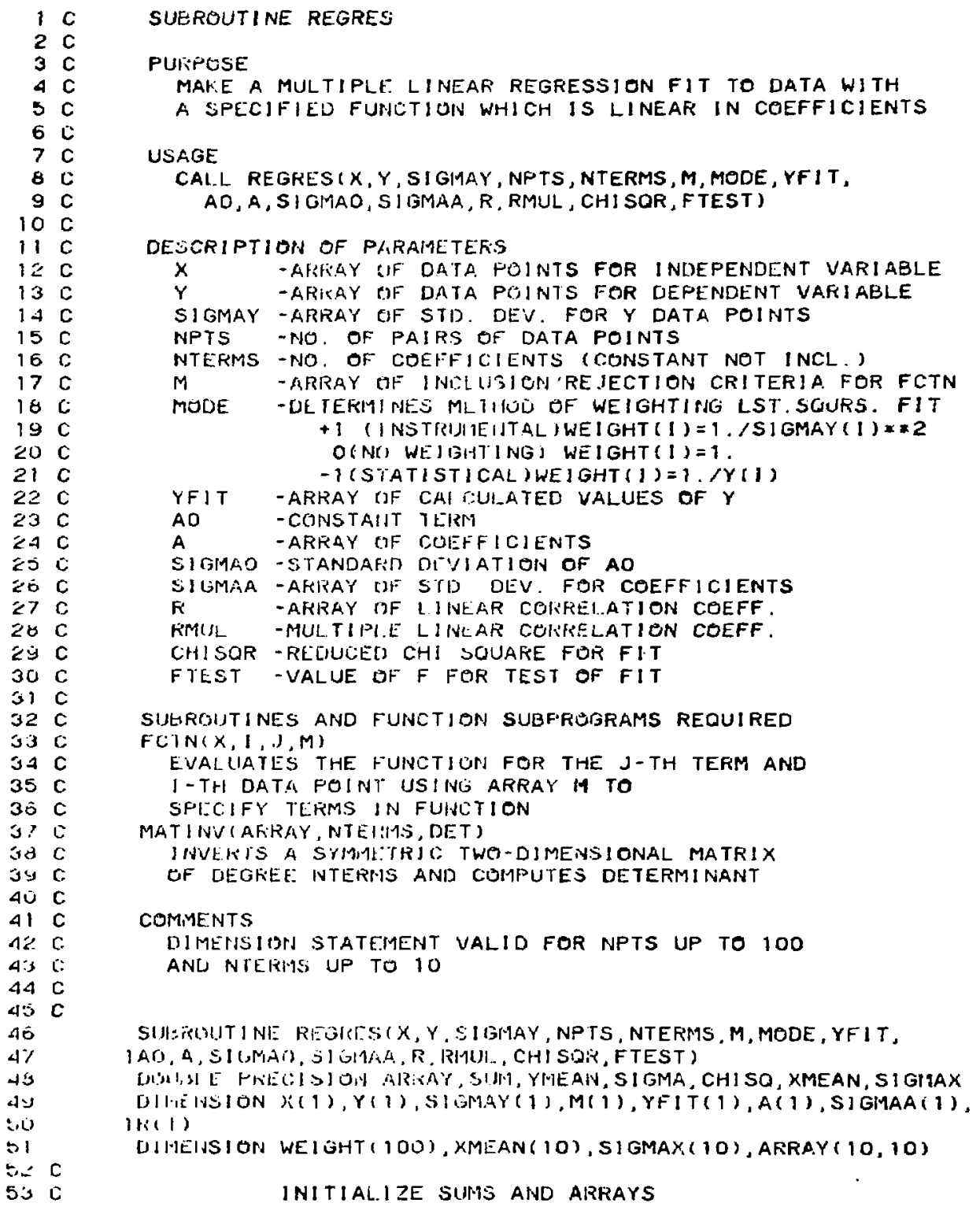




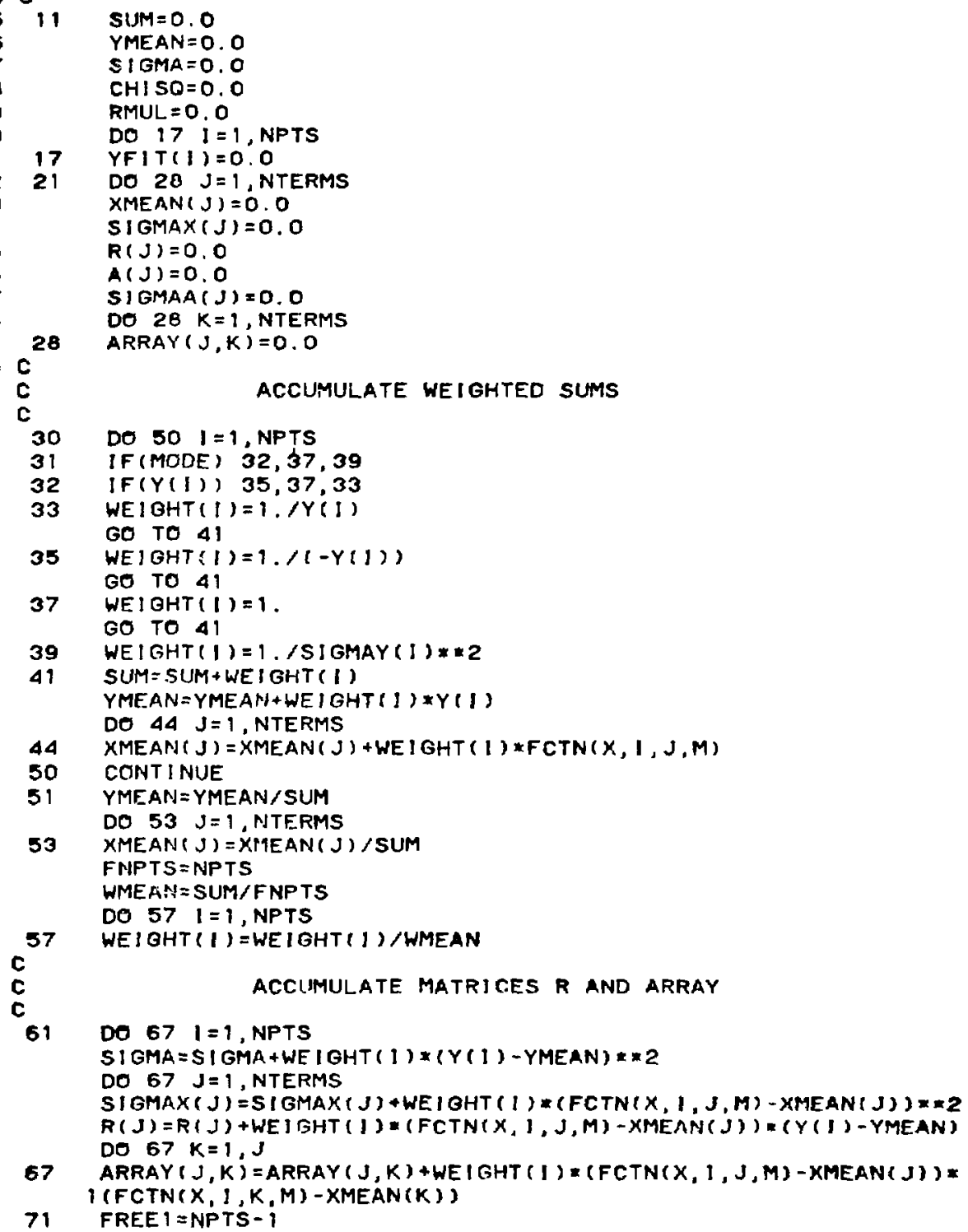




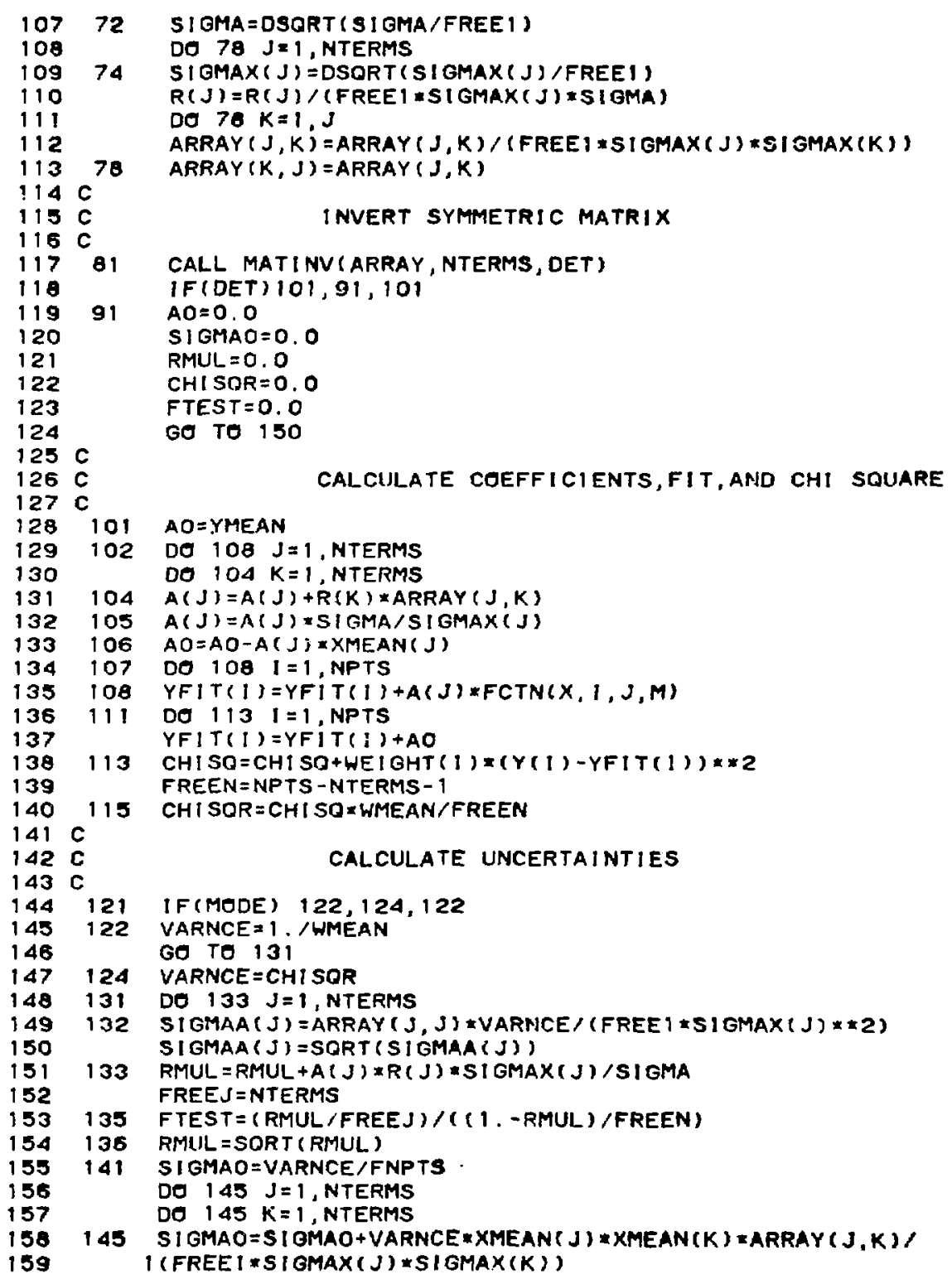


PIN.PLSE

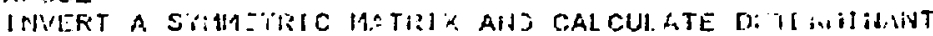
Usi it:

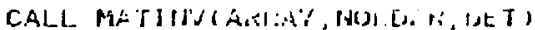

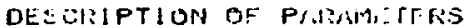

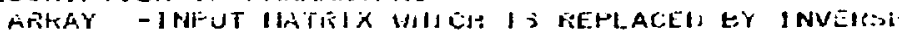

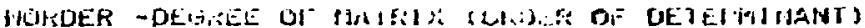

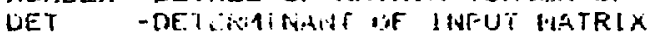

CUIMENTS

DIMENSION STATEMEINT VAI_JD FOR MORDER UP TE 10

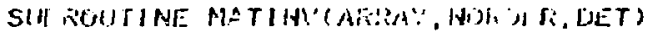

[Ur'U,

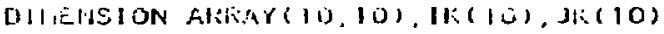

$n F-1=1$.

$00100 K=1$, NORDEF:

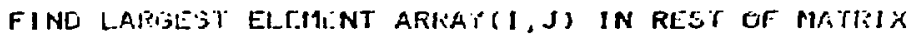

$A \mid W: K=0.0$

[ii) 30) $1=K$, NITHETER

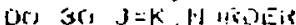

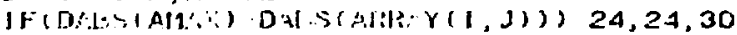

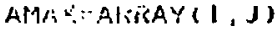

JKik; $=1$

Jl. $(K)=J$

CIHWTHILE

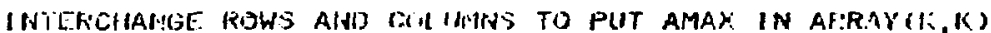

IF $(.9 M A X) 41,32,41$

IJE: $1=0.0$

GO TO 140

$41 \quad 1=1 k(k)$

IF $(1-K) 21,51,43$

43 DS $50 \mathrm{~J}=1$, NHADER

SAY:LE:ARIIAY $(K, J)$

A|kls.A $)^{\prime}(K, J)=A \mid R: A A(1, J)$

so AriTIAY $(1, J)=-$ SAVE

$51 \quad J=. K(K)$

if $F(J-K) 21,61,53$

53 DE $60 \quad 1=1$, NORDEIR

SAVE $=$ AFRAY $(I, K)$

AliliAY $(1, K)=$ ARRAY $(1, J)$

ARTIAY $(1, J)=-$ SAVE

\section{ACCUMULATE ELEMEIT:3 JF INVEIRSE MATRIX}

DO $70 \quad 1=1$, NORDER 

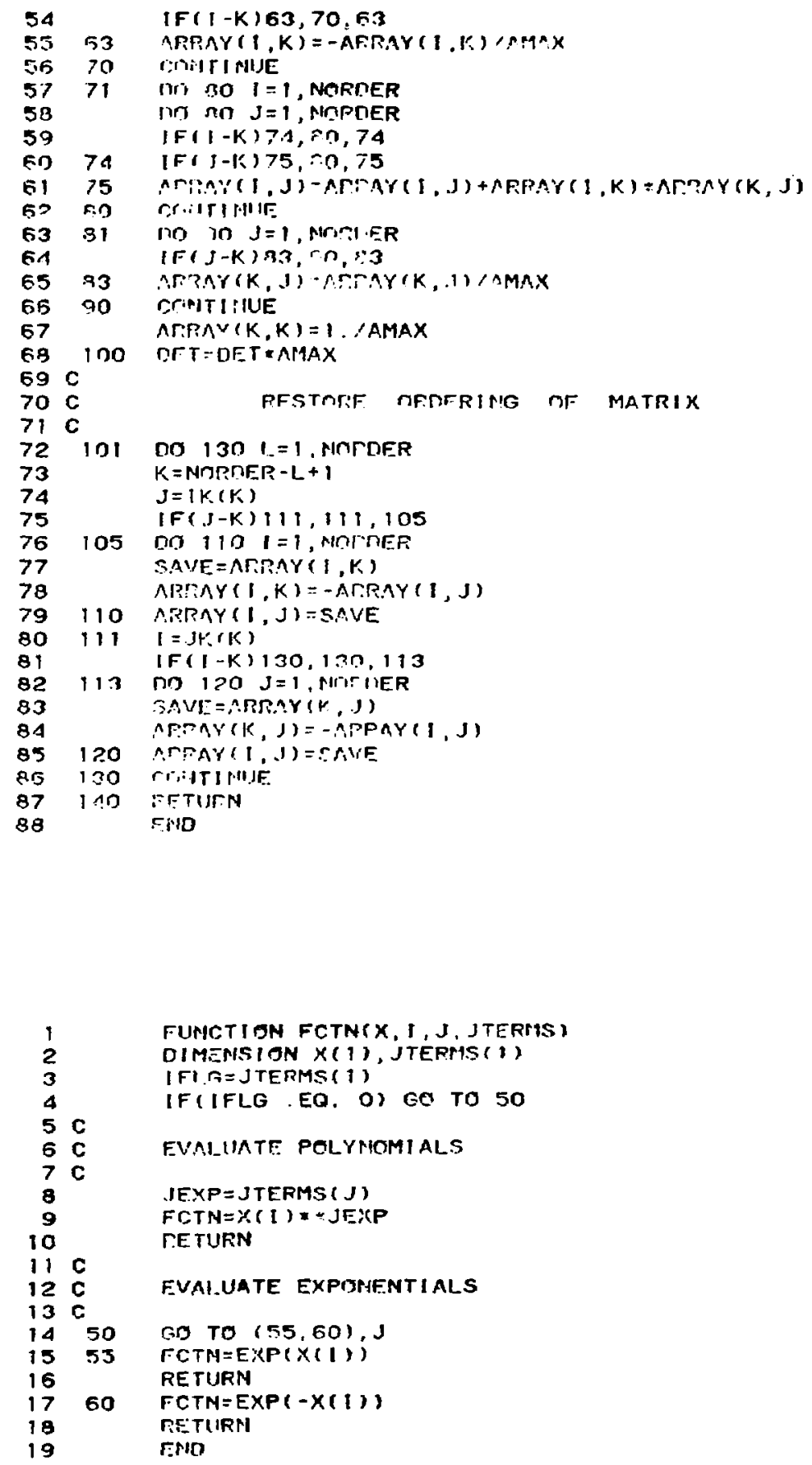


\section{APPENDIX H \\ MULTIPLE SURFACE DERIYATIONS \\ (MOMENTS OF SLAB TRANSMISSION SCORE)}

Given the formulations for the total score accumulation probability for the six cases presented in Chapter V [see Eqs. (5.1.1) through (5.1.6)], the $r$-th moment equations for the transmission score may be derived by following exactly the same procedure as was demonstrated in Chapter IV (and Appendix B) for the case of one splitting surface. A1though all the details are not repeated here, because of certain notational differences as well as the more varied combination of terms in the six cases referred to above, selected aspects of the derivation are presented. The reader should be able to supply the intermediate details needed to reconstruct the entire derivation with reference to Chapter IV and Apiendix B.

First, the $r$-th moment equations may be formulated by multiplying the score accumulation probabilities [Eqs. (5.1.1) through (5.1.6) by $s^{r}$ and integrating over ds, i.e.,

$$
M_{r, i}(\underline{P})=\int \psi_{i}(\underline{P}, s) s^{r} d s .
$$

By applying the Binomial Theorem [see Eq. (B.1.2a)] all cases in which a linear combination of scores appears in the argument of the $\psi$ function may be simplified and replaced by an appropriate sumation over $n$ moments $(\mathrm{n}=0,1,2, \cdots, \mathrm{r})$. For example, 


$$
\begin{aligned}
& \int \psi_{1}\left(\underline{P}, s^{\prime} s^{\prime}\right) s^{r} d s=\int \psi_{i}\left(\underline{P}, s^{*}\right)\left(s^{*}+s^{\prime}\right)^{r} d s^{*} \\
& =\sum_{n=0}^{r}\left(\begin{array}{l}
r \\
n
\end{array}\right) s^{-r-n} M_{n, j .}(P),
\end{aligned}
$$

where $s^{*}=s^{-} s^{\prime}$. Also, the Binomial Theorem is applied $\mathrm{m}_{i}$ times in treating the splitting term in the equations: first in integrating over $\mathrm{ds}$, then in integrating over $\mathrm{ds}_{\mathrm{m}_{i}-1}$, etc., and finally in integrating over ds ${ }_{1}$ (see Appendix B).

Collecting all terms in $\mathrm{M}_{r, i}$ leaves all the equations in a form similar to that of Eqs. (B.1.11-13), where all non-r-th moment terms are included in a source term $Q_{r, i}$. Substitution is then made for the score distribution functions. For the case of multiple splitting surfaces, these distributions are given as follows:

$$
\begin{aligned}
& \mathrm{p}_{S}(\underline{\mathrm{P}}, \mathrm{s})=\mathrm{p}_{\mathrm{E}, 0}(\underline{\mathrm{P}}, \mathrm{s})=\delta(\mathrm{s}), \\
& \mathrm{P}_{\mathrm{Sm}_{i}}(\underline{\mathrm{P}}, \mathrm{s})=\mathrm{p}_{\mathrm{R}_{i}}(\underline{\mathrm{P}}, \mathrm{s})=\delta(\mathrm{s}) \quad \text { for } \quad i=1,2, \cdots, N
\end{aligned}
$$

and $\mathrm{P}_{\mathrm{E}, \mathrm{D}}(\underline{\mathrm{P}}, \mathrm{s})=\delta(s-\mathrm{w})$.

In other words, only a back-surface escape results in a non-zero score. Finally, after substituting for the appropriate probability densities and kernels (see definitions at the beginning of Chapters IV and V) and performing the indicated Integrations, Eqs. (H.0.4)-(H.0.9) resu1t for the r-th moment of the transmission score (for the six cases of particle starting coordinate $z$ ). Use is also made of identity (B.2.2) in writing 
these equations with the transition kernel $\mathrm{k}\left(z, \mu \rightarrow z^{+}, \mu^{\prime \prime}\right)$. Also, the r-th moments for the different cases are represented by

$$
M_{r, i}(z, \mu, w) \text { for } z \text { in region } i \quad(i=1,2, \cdots, N+1)
$$

and

$$
M_{r, z_{i}}\left(z_{i}, \mu, w\right) \quad \text { for } \quad z=z_{i} \quad(1=1,2, \cdots, N) \text {. }
$$

In region 1 , for $0 \leq z<z_{1}$ :

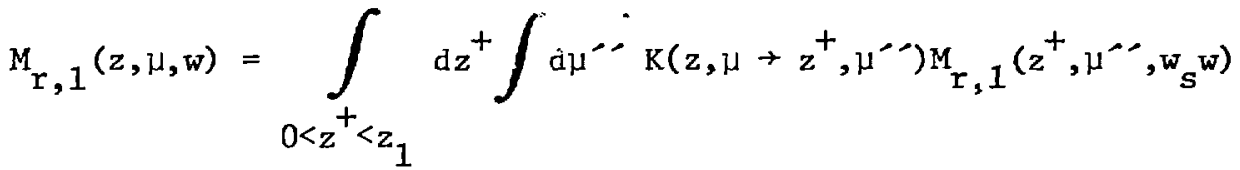

$$
\begin{aligned}
& +H(\mu) T\left(z+z_{1}, \mu\right) \cdot m_{1} \cdot M_{r, z_{1}}\left(z_{1}, \mu, w / m_{1}\right)+Q_{r, 1}(z, \mu, w),
\end{aligned}
$$

where

$$
\begin{gathered}
\mathrm{Q}_{1,1}(z, \mu, w)=0, \\
\mathrm{Q}_{2,1}(\mathrm{z}, \mu, \mathrm{w})=\mathrm{H}(\mu) \cdot \mathrm{m}_{1}\left(\mathrm{~m}_{1}-1\right) \cdot \mathrm{T}\left(\mathrm{z} \rightarrow \mathrm{z}_{1}, \mu\right)\left\{\mathrm{M}_{1, z_{1}}\left(\mathrm{z}_{1}, \mu, \mathrm{w} / \mathrm{m}_{1}\right)\right\}^{2} \cdot(\mathrm{H.0.4c})
\end{gathered}
$$

On surface 1 , for $z=z_{1}$ :

$$
\begin{aligned}
& M_{r, z_{1}}\left(z_{1}, \mu, w\right)=\int_{0<z^{+}<z_{1}} d^{+} \int d \mu^{\prime} k\left(z_{1}, \mu+z^{+}, \mu^{\prime \prime}\right) M_{r_{1}, 1}\left(z^{+}, \mu^{\prime}, w_{s} w\right) \\
& \left.+\int_{z_{1}<z^{+}<z_{2}} \mathrm{dz} z^{+} \int \mathrm{d} \mu^{-\infty} \mathrm{K}\left(z_{1}, \mu+\mathrm{z}^{+}, \mu^{-\infty}\right) \mathrm{M}_{\mathrm{r}, 2} ! z^{+}, \mu^{\cdots}, \mathrm{w}_{\mathrm{s}} \mathrm{w}\right) \\
& +H(\mu) T\left(z_{1}+z_{2}, \mu\right) \cdot m_{2} \cdot M_{r, z_{2}}\left(z_{2}, \mu, w / m_{2}\right)+Q_{r_{,} z_{1}}\left(z_{1}, \mu, w\right),(H .0 .5 a)
\end{aligned}
$$


where

$$
\begin{gathered}
\mathrm{Q}_{1, z_{1}}\left(\mathrm{z}_{1}, \mu, \mathrm{w}\right)=0 \\
\mathrm{Q}_{2, \mathrm{z}_{1}}\left(\mathrm{z}_{1}, \mu, \mathrm{w}\right)=\mathrm{H}(\mu) \cdot \mathrm{m}_{2}\left(\mathrm{~m}_{2}-1\right) \cdot \mathrm{T}\left(\mathrm{z}_{1} \rightarrow \mathrm{z}_{2}, \mu\right)\left\{\mathrm{M}_{1, \mathrm{z}_{2}}\left(\mathrm{z}_{2}, \mu, \mathrm{w} / \mathrm{m}_{2}\right)\right\}^{2}
\end{gathered}
$$

(H.0.5c)

In region $i$, for $z_{i-1}<z<z_{i}$ :

$$
\begin{aligned}
& M_{r, i}(z, \mu, w)=\int_{z_{i-1}^{<z^{+}<z_{i}}} d z^{+} \int \mathrm{d} \mu^{-\prime} K\left(z, \mu \rightarrow z^{+}, \mu^{-\prime}\right) M_{r, i}\left(z^{+}, \mu^{-\prime}, w_{s} w^{w}\right) \\
& +H(\mu) T\left(z \rightarrow z_{i}, \mu\right) \cdot n_{i} \cdot M_{r, z_{i}}\left(z_{i}, \mu, w / m_{i}\right)+[1-H(\mu)] \\
& x T\left(z \rightarrow z_{i-1}, \mu\right) \cdot \xi_{m_{i-1}} \cdot M_{r, z}{ }_{i-1}\left(z_{i-1}, \mu, w / \xi_{m_{i-1}}\right)+Q_{r, i}(z, \mu, w),
\end{aligned}
$$

where

$$
\begin{gathered}
Q_{1, i}(z, \mu, w)=0 \\
(H, 0.6 b) \\
Q_{2, i}(z, \mu, w)=H(\mu) \cdot m_{i}\left(m_{i}-1\right) \cdot T\left(z+z_{i}, \mu\right)\left\{M_{1, z_{i}}\left(z_{i}, \mu, w / m_{i}\right)\right\}^{2} \cdot(H .0 .6 c)
\end{gathered}
$$

On surface $i$, for $z=z_{i}$ : 


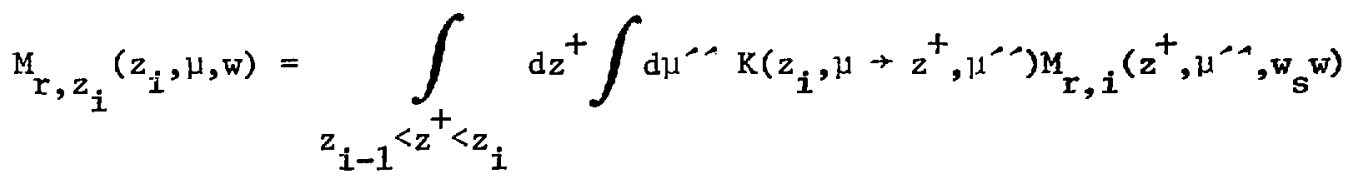

$$
\begin{aligned}
& +\int_{z_{i}<z^{+}<z_{i+1}} d z^{+} \int d \mu^{\prime \prime} k\left(z_{i}, \mu \rightarrow z^{+}, \mu^{\prime \prime}\right) M_{r, i+1}\left(z^{+}, \mu^{\prime \prime}, w_{s}, w\right) \\
& +[1-H(\mu)] T\left(z_{i} \rightarrow z_{i-1}, \mu\right) \cdot \xi_{m_{i-1}} \cdot M_{r, z_{i-1}}\left(z_{i-1}, \mu, w / \xi_{m_{i-1}}\right) \\
& +H(\mu) \mathrm{T}\left(z_{i}+z_{i+1}, \mu\right) \cdot \mathrm{m}_{i+1} \cdot \mathrm{M}_{\mathbf{r}, z_{i+1}}\left(z_{i+1}, \mu, w / \mathrm{m}_{i+1}\right) \\
& +Q_{r, z_{i}}\left(z_{i}, \mu, w\right)
\end{aligned}
$$

where

$$
\begin{gathered}
Q_{1, z_{i}}\left(z_{i}, \mu, w\right)=0 \\
Q_{2, z_{i}}\left(z_{i}, \mu, w\right)=H(\mu) \cdot m_{i+1}\left(m_{i+1}-1\right) \cdot T\left(z_{i} \rightarrow z_{i+1}, \mu\right) \\
\times\left\{M_{1, z_{i+1}}\left(z_{i+1}, \mu, w / m_{i+1}\right)\right\}^{2} .
\end{gathered}
$$

On surface $N$, for $z=z_{N}$ : 


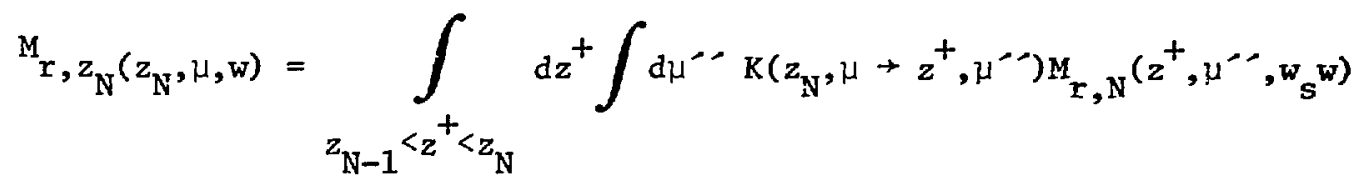

$$
\begin{aligned}
& +\int_{z_{N}<z^{+}<D} d z^{+} \int d \mu^{-} K\left(z_{N}, \mu \rightarrow z^{+}, \mu^{\prime \prime}\right) M_{r, N+1}\left(z^{+}, \mu^{\prime}, w_{s} w\right) \\
& +[1-\mathrm{H}(\mu)] \mathrm{T}\left(\mathrm{z}_{\mathrm{N}} \rightarrow \mathrm{z}_{\mathrm{N}-1}, \mu\right) \cdot \xi_{\mathrm{m}_{\mathrm{N}-1}} \cdot \mathrm{M}_{\mathrm{r}, \mathrm{z}_{\mathrm{N}-1}}\left(\mathrm{z}_{\mathrm{N}-1}, \mu, \mathrm{w} / \xi_{\mathrm{m}_{\mathrm{N}-1}}\right) \\
& +Q_{r, z_{N}}\left(z_{N}, \mu, w\right)
\end{aligned}
$$

where

$$
\begin{aligned}
& \mathrm{Q}_{1, z_{N}}\left(z_{N}, \mu, w\right)=H(\mu) T\left(z_{N} \rightarrow D, \mu\right) \cdot w \\
& Q_{2, z_{N}}\left(z_{N}, \mu, w\right)=H(\mu) T\left(z_{N} \rightarrow D, \mu\right) \cdot w^{2} .
\end{aligned}
$$

Finally in region $N+1$, for $z_{N}<z \leq D$ :

$$
\begin{aligned}
& M_{r, N+1}(z, \mu, w)=\int_{z_{N}<z^{+}<D} d z^{+} \int d \mu^{-\prime} k\left(z, \mu \rightarrow z^{+}, \mu^{-\prime}\right) M_{r, N+1}\left(z^{+}, \mu^{-\prime}, w_{s} w\right) \\
& +[1-H(\mu)] T\left(z \rightarrow z_{N}, \mu\right) \cdot \xi_{\mathrm{Bn}_{N}} \cdot M_{r, z_{N}}\left(z_{N}, \mu, w / \xi_{\mathrm{B}_{N}}\right)+Q_{r, N+1}(z, \mu, w),
\end{aligned}
$$

where

$$
Q_{1, N+1}(z, \mu, w)=H(\mu) T(z+D, \mu) \cdot w,
$$




$$
\mathrm{Q}_{2, \mathrm{~N}+1}(z, \mu, \mathrm{w})=\mathrm{H}(1) \mathrm{T}(\mathrm{z}+\mathrm{D}, \mu) \cdot \mathrm{w}^{2} .
$$

From this point onward, the first and second moments will be treated separately. The continuity/discontinuity conditions will be derived at the general (i-th) splitting surface. Finally, the equations will be presented in integro-differential form.

\section{H.1 The First Moment $(r:=1)$}

The purpose of this section is to demonstrate that the first moment of the transmission score is continuous across the general (i-th) splitting surface (as in the special case of a single splitting surface). Also, after all the proper substitutions are made, it is found that the coupled first moment equations reduce to a single formulation which holds over the entire slab.

First we consider Eqs. (H.0.6a,b) and Eqs. (H.0.7a,b) for $r=1$. Recalling the property that constant weight multipliers are separable in the moments (see Appendix D), it is possible to substitute Eqs. (H.0.7a,b) into Eq. (H.0.6a). Thus, for $r=1$ and $z$ in region $i$ :

$$
\begin{aligned}
& M_{1, i}(z, \mu, w)=w s \cdot \int d z^{+} \int d \mu^{\prime} K\left(z, \mu \rightarrow z^{+}, \mu^{\prime}\right) M_{1, i}\left(z^{+}, \mu^{\prime}, w\right) \\
& z_{i-1}<z^{+}<z_{i} \\
& +w_{S} \cdot \int_{z^{+}} d z^{+} \int \mathrm{d} \mu^{-} \mathrm{K}\left(z, \mu \rightarrow z^{+}, \mu^{-\cdots}\right) \mathrm{M}_{1,1+1}\left(z^{+}, \mu^{-\infty}, w\right) \\
& z_{i}<z^{+}<z_{i+1} \\
& +H(\mu) T\left(z \rightarrow z_{i+1}, \mu\right) M_{1, z_{i+1}}\left(z_{i+1}, \mu, w\right)+[1-H(\mu)] T\left(z \rightarrow z_{i-1}, \mu\right) \\
& \times M_{1, z_{1-1}}\left(z_{1-1}, \mu, w\right)
\end{aligned}
$$


In writing Eq. (H.1.1), the transmission probability $T\left(z \rightarrow z_{i}, \mu\right)$ has been absorbed either in the transition kernel $\mathrm{K}\left(\mathrm{z}_{i}, \mu \rightarrow \mathrm{z}^{+}, \mu^{-}\right)$or the transmission probability $\mathrm{T}\left(z_{i} \rightarrow z_{i+1}, \mu\right)$, as per Eq. (B.2.3).

We can write an equation for the first moment in region $1+1$ $\left(z_{i}<z<z_{i+1}\right)$ by analogy to Eq. (H.0.6a,b). Thus, for $r=1$ and weight multipliers separated:

$$
\begin{aligned}
& M_{1, i+1}(z, \mu, w)=w_{s} \cdot \int_{z_{i}<z^{+}<z_{i+1}} \mathrm{dz}^{+} \int \mathrm{d} \mu^{\prime} \mathrm{k}\left(z, \mu \rightarrow z^{+}, \mu^{\prime \prime}\right) M_{1, i+1}\left(z^{+}, \mu^{-}, w^{\prime}\right) \\
& +\mathrm{H}(11) \mathrm{T}\left(z \rightarrow z_{i+1}, \mu\right) \mathrm{M}_{1, z_{i+1}}\left(z_{i+1}, \mu, w\right) \\
& +[1-H(\mu)] \mathrm{T}\left(z \rightarrow z_{i}, \mu\right) M_{1, z_{i}}\left(z_{i}, \mu, w\right)
\end{aligned}
$$

The proper forms of Eqs. (H.0.7a,b) may be substituted into Eq. (H.I.2) to give

$$
\begin{aligned}
& M_{1, i+1}(z, \mu, w)=w_{s} \cdot \int_{z_{i}<z^{+}<z_{i+1}} d^{+} \int d^{\prime}-K\left(z, \mu \rightarrow z^{+}, \mu^{-\infty}\right) M_{1, i+1}\left(z^{+}, \mu^{-,}, w\right) \\
& +H(\mu) \mathrm{T}\left(z \rightarrow z_{i+1}, \mu\right) \mathrm{M}_{1, z_{i+1}}\left(z_{i+1}, \mu, w\right)+w_{s} \cdot \int_{z_{i-1}<z^{+}<z_{i}} \mathrm{dz}+\int \mathrm{d}^{-\cdots} \\
& \times \mathrm{K}\left(\mathrm{z}, \mu \rightarrow \mathrm{z}^{+}, \mu^{-}\right) \mathrm{M}_{1, i}\left(\mathrm{z}^{+}, \mu^{-}, \mathrm{w}\right)+[1-\mathrm{H}(\mu)] \mathrm{T}\left(\mathrm{z} \rightarrow \mathrm{z}_{i-1}, \mu\right) \\
& \times M_{1, z_{i-1}}\left(z_{i-1}, \mu, w\right)
\end{aligned}
$$


Again, the transmission probability $T\left(z \rightarrow z_{i}, \mu\right)$ has been absorbed by the transition kernel $\mathrm{K}\left(\mathrm{z}_{\mathrm{i}}, \mu \rightarrow \mathrm{z}^{+}, \mu^{\prime \prime}\right)$ and by the transmission probability $T\left(z_{i} \rightarrow z_{i-1}, \mu\right)$

As in Chapter IV (Appendix B), the limits of the first moment solution in regions $i$ and $i+1$ are defined

$$
\begin{aligned}
& M_{1}{ }^{-}\left(z_{i}, \mu, w\right) \equiv \lim _{z \rightarrow z_{i-}} M_{1, i}(z, \mu, w) \\
& \text { and } \quad \mathrm{M}_{1}^{+}\left(z_{i}, \mu, w\right) \equiv \lim _{z \rightarrow z_{i+}} M_{1, i+1}(z, \mu, w)
\end{aligned} .
$$

Then, if the limit of Eq. (H.1.1) is taken as the starting location $z$ is allowed to approach the splitting surface $z_{\dot{i}}$ from below, and if the limit of Eq. (H.1.3) is taken as $z$ approaches $z_{i}$ from above, we find that

$$
M_{1}{ }^{-}\left(z_{i}, \mu, w\right)=M_{1}^{+}\left(z_{i}, \mu, w\right)=M_{1, z_{i}}\left(z_{i}, \mu, w\right)
$$

By Eq. (H.1.5), the first moment of the transmission score is continuous across any splitting surface $z_{i^{*}}$ This, of course, is a generalization of the condition that was found in Chapter IV for only a single splitting surface in the geometry. Equation (H.1.5) was derived specifically for a surface at $z_{i}$ which has other splitting surfaces on either side, i.e., at $z_{i-1}$ and $z_{i+1}$. The same relationship may be derived for the case of surfaces $1\left(\right.$ at $\left.z_{1}\right)$ and $N\left(\right.$ at $\left.z_{N}\right)$, both of which have a neighboring splitting surface on one side, but an escape surface on the other. In these cases, the derivation proceeds by starting with Eqs. $(\mathrm{H} .0 .4,5)$ or Eqs. (H.0.8,9), rather than as done above with Eqs. (H.0.6,7). 
If one were to start with the first moment formulation written for any of the $\mathrm{N}+1$ regions in the slab geometry [for example, Eq. (H.0.6) for region i], and appropriately substitute (recursively) for all the $M_{1}$ terms which couple to the equation, the result would be an identical formulation of the first moment for all the starting regions $M_{1, j}$

$$
(j=1,2, \cdots, N+1) \text {, i.e. , }
$$

$$
\begin{aligned}
M_{1, j}(z, \mu, w) & =\sum_{i=1}^{N+1} w_{s} \cdot \int_{z_{i-1}<z^{+}<z_{i}} d^{+} \int d \mu^{-} K\left(z, \mu \rightarrow z^{+}, \mu^{-\rho}\right) M_{1, i^{\prime}}\left(z^{+}, \mu^{-}, w\right) \\
& +T(z \rightarrow D, \mu) \cdot w \quad \text { for } \quad z_{j-1} \leq z^{-}<z_{j}
\end{aligned}
$$

Equation (H.1.6) holds for all regions $j=1,2, \cdots, N+1$ (with the added definitions: $z_{0} \equiv 0$ and $\left.z_{N+1} \equiv D\right)$. Thus, when the equations are written in this manner, we see that the same equation governs the first moment solution, irrespective of the region in which the particle is assumed to be introduced. Formally, then, we define the first moment solution $M_{1}(z, \mu, w)$, such that (for $\left.i=1,2, \cdots, N+1\right)$ :

$$
M_{1}(z, \mu, w) \equiv M_{1, i}(z, \mu, w) \quad \text { for } \quad z_{i-1} \leq z<z_{i}
$$

Thus, Eq. (H.1.6) may be reduced to one simplified formulation over the entire slab $(0 \leq z \leq D)$ :

$$
\begin{gathered}
M_{1}(z, \mu, w)=w_{s} \int_{0<z^{+}<D}^{\infty} d^{+} \int d^{\prime} \cdot K\left(z, \mu \rightarrow z^{+}, \mu^{N}\right) M_{1}\left(z^{+}, \mu^{-\infty}, w\right) \\
+T(z \rightarrow D, \mu) \cdot w .
\end{gathered}
$$


The conversion of the integral equation (H.1.8) to integro-differential form has already been demonstrated [see Eqs. $(4.1 .2 .5)$ through (4.1.2.15)] with the result:

$$
\begin{gathered}
-\mu \frac{\partial}{\partial z} F_{1}(z, \mu, w)+\Sigma_{T} F_{1}(z, \mu, w)=\int d \mu-\Sigma_{S} \cdot E\left(z, \mu \rightarrow \mu^{\prime \prime}\right) F_{1}\left(z, \mu^{\prime \prime}, w\right) \\
\quad+H(\mu) \delta(z-D) \cdot \frac{\mu}{\Sigma_{T}} \cdot w,
\end{gathered}
$$

where

$$
\mathrm{F}_{1}(z, \mu, \mathrm{w}) \equiv 1 / \Sigma_{\mathrm{T}} \cdot \mathrm{M}_{1}(z, \mu, w)
$$

Thus, as before, the effects of splitting, Russian roulette, and survir 1 biasing are totally absent from the first moment equation.

\section{H. 2 The Second Moment $(r=2)$}

In this section, the discontinuity at the general (i-th) splitting surface is characterized. It is demonstrated that the discontinuity condition is identical to the condition found for the case of a single splitting surface (Chapter IV).

We now consider the second moment of the transmission score. As in Section H.1, the proper forms $(r=2)$ of Eqs. $(H .0 .7 a, c)$ are substituted into $\mathrm{Eq} .(\mathrm{H} .0 .6 \mathrm{a}, \mathrm{c})$ after the property of weight separability is applied to both sets of equations. [The substitution is made for $\left.H(\mu) M_{2, z_{i}}\left(z_{i}, \mu, w\right).\right]$ The following equation results: 


$$
\begin{aligned}
& M_{2, i}(z, \mu, w)=w_{s}^{2} \cdot \int d z^{+} \int d \mu^{-} k\left(z, \mu+z^{+}, \mu^{-}\right) M_{2, i}\left(z^{+}, \mu^{-}, w\right) \\
& z_{i-1}<z^{+}<z_{i} \\
& +\left(\frac{w_{s}^{2}}{m_{i}}\right) \cdot \int_{z_{i}<z^{+}<z_{i+1}} d z^{+} \int d \mu^{\cdots} K\left(z, \mu \rightarrow z^{+}, \mu^{-}\right) M_{2, i+1}\left(z^{+}, \mu^{-\cdots}, w\right) \\
& +H(\mu)\left(\frac{1}{m_{i} m_{i+1}}\right) \cdot T\left(z \rightarrow z_{i+1}, \mu\right) M_{2, z_{i+1}}\left(z_{i+1}, \mu, w\right)+H(\mu)\left(\frac{m_{i+1}-1}{m_{i} m_{i+1}}\right) \\
& \times \mathrm{T}\left(\mathrm{z} \rightarrow \mathrm{z}_{i+1}, \mu\right)\left\{\mathrm{M}_{1, z_{i+1}}\left(z_{i+1}, \mu, w\right)\right\}^{2}+[1-H(\mu)] \cdot \mathrm{m}_{i-1} \\
& \times T\left(z \rightarrow z_{i-1}, \mu\right) M_{2, z_{i-1}}\left(z_{i-1}, \mu, w\right)+H(\mu) \cdot\left(\frac{m_{i}-1}{m_{i}}\right) \\
& \times T\left(z \rightarrow z_{i}, \mu\right) \cdot\left\{M_{1, z_{i}}\left(z_{i}, \mu, w\right)\right\}^{2} .
\end{aligned}
$$

An equation for $z$ in region $i+1$ may be written $(r=2)$ by analogy to Eq. (H.0.6a,c) and by applying the property of weight separability:

$$
\begin{aligned}
& M_{2,1+1}(z, \mu, w)=w_{s}^{2} \cdot \int_{z_{i}<z^{+}<z_{i+1}} d_{z}^{+} \int d \mu \cdots K\left(z, \mu+z^{+}, \mu^{\prime \prime}\right) M_{2, i+1}\left(z^{+}, \mu^{\cdots}, w\right) \\
& +H(\mu)\left(\frac{1}{m_{i+1}}\right) T\left(z \rightarrow z_{i+1}, \mu\right) M_{2, z_{i+1}}\left(z_{i+1}, \mu, w\right)+[1-H(\mu)] T\left(z \rightarrow z_{i}, \mu\right) \\
& \quad \times m_{1} \cdot M_{2, z_{i}}\left(z_{i}, \mu, w\right)+H(\mu)\left(\frac{m_{i+1}-1}{m_{i+1}}\right) T\left(z \rightarrow z_{i+1}, \mu\right) \\
& \quad \times\left\{M_{1, z_{1+1}}\left(z_{i+1}, \mu, w\right)\right\}^{2}
\end{aligned}
$$


Substitution of Eq. $(H .0 .7 a, c)$ for $[1-H(\mu)] M_{2, z_{1}}\left(z_{1}, \mu, w\right)$ in Eq. (H.2.2) yields

$$
\begin{aligned}
& M_{2, i+1}(z, \mu, w)=w_{s}^{2} \cdot \int_{z_{i}<z^{+}<z_{i+1}} d^{+} \int d \mu^{--} k\left(z, \mu \rightarrow z^{+}, \mu^{--}\right) M_{2, i+1}\left(z^{+}, \mu^{-}, w\right) \\
& +H(\mu)\left(\frac{1}{m_{i+1}}\right) T\left(z \rightarrow z_{i+1}, \mu\right) M_{2, z_{1+1}}\left(z_{i+1}, \mu, w\right)+m_{1} w_{s}^{2} \cdot \int d^{+} \\
& z_{i-1}<z^{+}<z_{i} \\
& \times \int \mathrm{d} \mu^{\cdots} \mathrm{K}\left(z, \mu+z^{+}, \mu^{\prime}\right) \mathrm{M}_{2, i^{\prime}}\left(z^{+}, \mu^{-}, w\right)+[1-H(\mu)] \mathrm{m}_{i} \cdot \mathrm{m}_{i-1} \\
& \times T\left(z+z_{i-1}, \mu\right) M_{2, z_{i-1}}\left(z_{i-1}, \mu, w\right)+H(\mu)\left(\frac{m_{i+1}-1}{m_{i+1}}\right) \\
& x T\left(z+z_{i+1}, \mu\right)\left\{M_{1, z_{i+1}}\left(z_{i+1}, \mu, w\right)\right\}^{2} .
\end{aligned}
$$

As in the case for the first moment, the following limits may be defined:

$$
\begin{aligned}
& M_{2}{ }^{-}\left(z_{i}, \mu, w\right) \equiv \lim _{z \rightarrow z_{i-}} M_{2, i}(z, \mu, w) \\
& M_{2}^{+}\left(z_{i}, \mu, w\right) \equiv \lim _{z \rightarrow z_{i+}} M_{2, i+1}(z, \mu, w) .
\end{aligned}
$$

Then, upon taking the IImit of Eq. (H.2.1) as $z$ approaches $z_{1}$ from below and the limit of Eq. (H.2.3) as $z$ approaches $z_{1}$ from above, the discontinuity condition becomes apparent:

for $\underline{\mu<0 \text { : }}$

$$
M_{2}^{-}\left(z_{1}, \mu, w\right)=\frac{1}{m_{1}} M_{2}^{+}\left(z_{1}, \mu, w\right),
$$


and for $\underline{\mu}>0$ :

$$
M_{2}{ }^{+}\left(z_{i}, \mu, w\right)=m_{i} \cdot M_{2}^{-}\left(z_{i}, \mu, w\right)-\left(m_{i}-1\right) \cdot\left\{M_{1_{s}} z_{i}\left(z_{i}, \mu, w\right)\right\}^{2} \cdot(H .2 .5 b)
$$

The discontinuity characterized in Eqs. (H.2.5) is a generalization of the condition occurring in the case of a single splitting surface [see Eqs. (4.1.1.13,14)]. Although Eqs. (H.2.5) strictly apply only for the i-th splitting surface which has other splitting surfaces on either side, the same discontinuity can be derived at $z_{1}$ and $z_{N}$. In these latter cases, however, the derivation would proceed by starting with Eqs. (H.0.4,5) or (H.0.8,9), rather than with Eqs. (H.0.6,7).

The integral second moment equation in each region can be converted to an integro-differential form which is more amenable to standard solution techniques. The conversion follows along the same lines as presented in Chapter IV (Section 4.1.2).

For particles beginning their next flight in region 1, Eqs. (H.0.4a,c) may be rewritten after first breaking up the transition kernel [see Eq. (B.2.2)]:

$$
\begin{aligned}
M_{2,1}(z, \mu, w) & =w_{s}^{2} \cdot \int_{0<z^{+}<z_{1}} d^{+} \int d \mu^{-}-\frac{\Sigma_{T}}{|\mu|} T\left(z \rightarrow z^{+}, \mu\right) E\left(z^{+}, \mu \rightarrow \mu^{-}\right) \\
& \times M_{2,1}\left(z^{+}, \mu^{-}, w\right)+S_{2,1}(z, \mu, w),
\end{aligned}
$$

where 


$$
\begin{gathered}
S_{2,1}(z, \mu, w)=H(\mu) T\left(z+z_{1}, \mu\right) \cdot\left(\frac{1}{m_{1}}\right) \cdot M_{2, z_{1}}\left(z_{1}, \mu, w\right)+H(\mu)\left(\frac{m_{1}-1}{m_{1}}\right) \\
\quad \times T\left(z \rightarrow z_{1}, \mu\right)\left\{M_{1, z_{1}}\left(z_{1}, \mu, w\right)\right\}^{2} .
\end{gathered}
$$

Again, in writing Eqs. (H.2.6) and (H.2.7) the weight-multipliers are separated from the moments. The conversion of Eq. (H.2.6) to integrodifferential form follows the same procedure as applied to Eq. (4.1.2.18) in converting the latter equation to $\mathrm{Eq} \cdot(4.1 .2 .23)$. Applying the same steps to Eq. (H.2.6), the following equation is derived:

$$
\begin{aligned}
&-\mu \frac{\partial}{\partial z} F_{2,1}(z, \mu, w)+\Sigma_{T} F_{2,1}(z, \mu, w)=\int \mathrm{d} \mu \Sigma_{S}^{*} \cdot E\left(z, \mu \rightarrow \mu^{-\prime}\right) \\
& \times F_{2,1}\left(z, \mu^{-\prime}, w\right)+H(\mu) \delta\left(z-z_{1}\right) \frac{\mu}{\Sigma_{T}} \\
& \times\left[\frac{1}{m_{1}} \cdot M_{2, z_{1}}\left(z_{1}, \mu, w\right)+\left(\frac{m_{1}-1}{m_{1}}\right) \cdot\left\{M_{1, z_{1}}\left(z_{1}, \mu, w\right)\right\}\right.2],
\end{aligned}
$$

where

$$
F_{2,1}(z, \mu, w) \equiv 1 / \Sigma_{T} \cdot M_{2,1}(z, \mu, w)
$$

and

$$
\Sigma_{\mathrm{s}}^{*} \equiv \mathrm{w}_{\mathrm{s}}^{2} \Sigma_{\mathrm{T}}
$$

For particles beginning their next flight in an interior region (i), Eqs. (H.0.6a, C) may be rewritten for $r=2$ as 


$$
\begin{gathered}
M_{2, i}(z, \mu, w)=w_{s}^{2} \cdot \int_{z_{i-1}^{<z^{+}<z_{i}}} d z^{+} \int d \mu^{-} \frac{\Sigma_{T}}{|\mu|} T\left(z \rightarrow z^{+}, \mu\right) \Sigma\left(z^{+}, \mu \rightarrow \mu^{-\infty}\right) \\
\times M_{2, i}\left(z^{+}, \mu^{-}, w\right)+s_{2, i}(z, \mu, w),
\end{gathered}
$$

where

$$
\begin{aligned}
S_{2, i}(z, \mu, w)= & H(\mu) T\left(z \rightarrow z_{i}, \mu\right) \cdot\left(\frac{1}{m_{i}}\right) \cdot M_{2, z_{i}}\left(z_{i}, \mu, w\right)+[1-H(\mu)] \\
& \times T\left(z \rightarrow z_{i-1}, \mu\right) \cdot m_{i-1} \cdot M_{2, z_{i-1}}\left(z_{i-1} ; \mu, w\right)+H(\mu)\left(\frac{m_{i}-1}{m_{i}}\right) \\
& \times T\left(z \rightarrow z_{i}, \mu\right)\left\{M_{1, z_{i}}\left(z_{i}, \mu, w\right)\right\}^{2} .
\end{aligned}
$$

Again, the same procedure is followed in converting Eq. (H.2.9) as was followed for $M_{2,1}$ in $E_{q}$. (4.1.2.18), except that $M_{2, i}$ replaces $M_{2,1}$ in the equations and all integrations over $\mathrm{dz}^{+}$are now restricted to the range of starting locations $z_{i-1}<z^{+}<z_{i}$. The term $S_{2, i}$ also replaces $\mathrm{S}_{2,1}$ in the conversion. The resulting integro-differential equation is written:

$$
\begin{aligned}
& -\mu \frac{\partial}{\partial z} F_{2, i}(z, \mu, w)+\Sigma_{T} F_{2, i}(z, \mu, w)=\int d \mu \cdots \Sigma_{s}^{*} \cdot E\left(z, \mu \rightarrow \mu^{-\prime}\right) F_{2, i}\left(z, \mu^{\prime}, w\right) \\
& \quad+H(\mu) \delta\left(z-z_{i}\right) \cdot \frac{\mu}{\Sigma_{T}} \cdot\left[\frac{1}{m_{i}} \cdot M_{2, z_{i}}\left(z_{i}, \mu, w\right)+\left(\frac{m_{i}-1}{m_{i}}\right)\right. \\
& \left.\quad \times\left\{M_{1, z_{i}}\left(z_{i}, \mu, w\right)\right\}^{2}\right]+[1-H(\mu)] \delta\left(z-z_{i-1}\right) \cdot \frac{|\mu|}{\Sigma_{T}} \cdot m_{i-1} \\
& \quad \times M_{2, z_{i-1}}\left(z_{i-1}, \mu, w\right)
\end{aligned}
$$


where

$$
\mathrm{F}_{2, i}(z, \mu, w) \equiv 1 / \Sigma_{\mathrm{T}} \cdot \mathrm{M}_{2, i}(z, \mu, w)
$$

Finally, for particles beginning their next flight in region $\mathrm{N}+1$, Eqs. (H.0.9a,c) are rewritten here for $r=2$ :

$$
\begin{gathered}
M_{2, N+1}(z, \mu, w)=w_{S^{2}}^{2} \cdot \int_{z_{N}<z^{+}<D} d^{+} \int d \mu^{\prime} \frac{\sum_{T}}{|\mu|} T\left(z \rightarrow z^{+}, \mu\right) E\left(z, \mu \rightarrow \mu^{\prime}\right) \\
\quad \times M_{2, N+1}\left(z^{+}, \mu^{-}, w\right)+S_{2, N+1}(z, \mu, w),
\end{gathered}
$$

where

$$
\begin{aligned}
& S_{2, N+1}(z, \mu, w)=[1-H(\mu)] T\left(z \rightarrow z_{N}, \mu\right) \cdot m_{N} \cdot M_{2, z_{N}}\left(z_{N}, \mu, w\right) \\
& +H(\mu) T(z \rightarrow D, \mu) \cdot w^{2} .
\end{aligned}
$$

Following the same conversion steps as before, the integro-diffe:-ential form of the above equation [Eq. (H.2.12)] becomes

$$
\begin{aligned}
& -\mu \frac{\partial}{\partial z} F_{2, N}(z, \mu, w)+\Sigma_{T^{\prime}} T_{2, N}(z, \mu, w)=\int d \mu^{\prime \prime} \Sigma_{s}^{*} \cdot E\left(z, \mu \rightarrow \mu^{\prime \prime}\right) F_{2, N}\left(z, \mu^{\prime}, w\right) \\
& \quad+[1-H(\mu)] \delta\left(z-z_{N}\right) \frac{|\mu|}{\Sigma_{T}} \cdot m_{N} \cdot M_{2, z_{N}}\left(z_{N}, \mu, w\right) \\
& \quad+H(\mu) \delta(z-D) \cdot \frac{\mu}{\Sigma_{T}} \cdot w^{2},
\end{aligned}
$$

where

$$
F_{2, N}(z, \mu, w) \equiv 1 / \Sigma_{T} M_{2, N}(z, \mu, w)
$$


Inspection of Eqs. (H.2.8), (H.2.11), and (H.2.14) indicates that the homogeneous forms of these equations are all equivalent. In fact (as in Chapter IV for a two-region problem), one integro-differential equation may be written over the entire slab for all starting locations not on the splitting surfaces, i.e., $z \neq z_{i}(i=1,2, \cdots, N)$ :

$$
\begin{aligned}
& -\mu \frac{\partial}{\partial z} F_{2}(z, \mu, w)+\Sigma_{T} F_{2}(z, \mu, w)=\int d \mu^{\prime \prime} \Sigma_{S}^{*} \cdot E\left(z, \mu \rightarrow \mu^{\prime \prime}\right) F_{2}\left(z, \mu^{\prime \prime}, w\right) \\
& \quad+H(\mu) \delta(z-D) \cdot \frac{\mu}{\Sigma_{T}} \cdot w^{2},
\end{aligned}
$$

where

$$
\mathrm{F}_{2}(z, \mu, w) \equiv 1 / \Sigma_{\mathrm{T}} \mathrm{M}_{2}(z, \mu, w)
$$

and

$$
\Sigma_{\mathrm{S}}^{*} \equiv \mathrm{w}_{\mathrm{S}}^{2} \Sigma_{\mathrm{T}}
$$

Moreover, the internal boundary sources at each splitting surface, represented by the delta-funcrion sources in Eqs. (H.2.8, 11, 14) are accounted for by imposing the appropriate discontinuity on the second moment solution at $z=z_{i}(i=1,2, \cdots, N)$. This discontinuity was characterized in Eqs. (H.2.5a,b) for both positive and negative directions. 
APPENDIX I

MULTIPLE SURFACE DERIVATIONS

(TIME PER PARTICLE HISTORY)

As stated in Section 5.2, the development of the equations governing the time per particle history $\left[\tau_{p}(z, \mu)\right]$ for the case of multiple surfaces follows along the same lines as the derivation presented in Section 4.2 (for the single splitting surface). Of course, the formulations are now complicated by the fact that with $N$ surfaces and $N+1$ regions, the equations from case to case exhibit a different ser of terms due to the particular set of next events characterizing a given formulation.

Starting with the six cases represented by Eqs. (5.2.7) through (5.2.12), the next step in the development would be to substitute the appropriate probability kernels (see definitions at the beginning of Section 5.2) and the score distribution functions [Eq. (5.2.1)]. After performing most of the indicated integrations, the procedure yields a set of equations written explicitly with respect to $(z, \mu)$ coordinates. As an example, the substitution and integration procedure, when applied to Eq. $(5.2 .7 \mathrm{a}, \mathrm{b})$, yields the following equation for particles starting their next flight in region $i\left(z_{i-1}<z<z_{i}\right)$ :

(see next page) 


$$
\begin{aligned}
& \tau_{p, i}(z, \mu)=\int_{z_{i-1}^{<z^{+}<z_{i}}} \mathrm{dz} z^{+} \int \mathrm{d} \mu^{-1} \mathrm{k}\left(z, \mu \rightarrow z^{+}, \mu^{-\infty}\right) \tau_{p, i}\left(z^{+}, \mu^{-\infty}\right) \\
& +[1-H(\mu)] \cdot\left(\frac{1}{m_{1-1}}\right) T\left(z+z_{1-1}, \mu\right) \tau_{p, z_{1-1}}\left(z_{1-1}, \mu\right) \\
& +H(\mu) \cdot m_{i} \cdot T\left(z+z_{i}, \mu\right) \tau_{p, z_{i}}\left(z_{i}, \mu\right)+\int_{z_{i-1}<z^{+}<z_{i}} d z^{+} \\
& \times \frac{\Sigma_{T}}{|\mu|} \mathrm{T}\left(z \rightarrow z^{+}, \mu\right) \cdot \tau_{c}+[1-H(\mu)] \cdot T\left(z \rightarrow z_{i-1}, \mu\right) \cdot \tau_{R, i-1} \\
& +\mathrm{H}(\mu) \mathrm{T}\left(z \rightarrow z_{i}, \mu\right) \cdot \tau_{s, i} \cdot
\end{aligned}
$$

Similarly, the substitution and integration procedure applied to Eq. (5.2.10a,b) yields the following equation for particles resuming their flight from surface $1\left(z=z_{i}\right)$ :

(see next page) 


$$
\begin{aligned}
& \tau_{p, z_{L}}\left(z_{i}, \mu\right)=\int d^{+} \int d \mu^{-} k\left(z_{1}, \mu+z^{+}, \mu^{-\prime}\right) \tau_{p, i}\left(z^{+}, \mu^{-\infty}\right) \\
& z_{i-1}<z^{+}<z_{1} \\
& +\int_{z_{i}<z^{+}<z_{i+1}} d z^{+} \int d \mu^{\prime \prime} k\left(z_{i}, \mu \rightarrow z^{+}, \mu^{\prime \prime}\right) \tau_{p, i+1}\left(z^{+}, \mu^{\prime \prime}\right) \\
& +[1-H(\mu)] \cdot\left(\frac{1}{m_{i-1}}\right) T\left(z_{i} \rightarrow z_{i-1}, \mu\right) \tau_{p, z_{1-1}}\left(z_{1-1}, \mu\right)+H(\mu) \\
& \times m_{i+1} \cdot T\left(z_{i} \rightarrow z_{i+1}, \mu\right) \tau_{p, z_{i+1}}\left(z_{i+1}, \mu\right)+\int_{z_{i-1}<z^{+}<z_{i}} d z^{+} \frac{\Sigma_{T}}{|\mu|} \\
& \times \mathrm{T}\left(z_{i} \rightarrow z^{+}, \mu\right) \cdot \tau_{c}+\int_{z_{i}<z^{+}<z_{i+1}} \mathrm{~d} z^{+} \frac{\Sigma_{\mathrm{T}}}{|\mu|} \mathrm{T}\left(z_{i} \rightarrow z^{+}, \mu\right) \cdot \tau_{c} \\
& +[1-H(\mu)] \cdot T\left(z_{i} \rightarrow z_{i-1}, \mu\right) \cdot \tau_{R, i-1}+H(\mu) T\left(z_{i}+z_{i+1}, \mu\right) \\
& \times \tau_{s, 1+1}
\end{aligned}
$$

Also, by analogy to $\mathrm{Eq.} \mathrm{(I.1),} \mathrm{the} \mathrm{following} \mathrm{equation} \mathrm{may} \mathrm{be} \mathrm{written}$ for particles beginning their next flight in region $1+1$, for

$z_{i}<z<z_{i+1}:$ 


$$
\begin{aligned}
& \tau_{p, i+1}(z, \mu)=\int_{z_{1}<z^{+}<z_{i+1}} d z^{+} \int d \mu^{\prime} k\left(z, \mu \rightarrow z^{+}, 1^{\prime \prime}\right) \tau_{p, i+1}\left(z^{+}, \mu^{\prime \prime}\right) \\
& +[1-H(\mu)] \cdot\left(\frac{1}{m_{i}}\right) \cdot T\left(z+z_{i}, \mu\right) \tau_{p, z_{i}}\left(z_{i}, \mu\right)+H(\mu) \cdot m_{i+1} \\
& \times T\left(z \rightarrow z_{i+1}, \mu\right) \tau_{p, z_{i+1}}\left(z_{i+1}, \mu\right)+\int_{z_{i}<z^{+}<z_{i+1}} d z+\frac{\Sigma_{T}}{|\mu|} \\
& x T\left(z \rightarrow z^{+}, \mu\right) \cdot \tau_{c}+[1-H(\mu)] T\left(z \rightarrow z_{i}, \mu\right) \cdot \tau_{R, i}+H(\mu) \\
& \times T\left(z \rightarrow z_{i+i}, \mu\right) \cdot \tau_{s, i+1} .
\end{aligned}
$$

The derivation of the discontinuity condition at each spitting surface proceeds in the same manner as already shown for the second moment of the transmission score (see Appendix H). To characterize the condition at the i-th surface, the expression for $\tau_{p, z_{i}}$ from Eq. (I.2) is substituted into the third term of Eq. (I.1). Recall that only terms in Eq. (I.2) which do not vanish for $\mu>0$ contribute in the substitution. Thus, Eq. (I.1) becomes: 


$$
\begin{aligned}
& \tau_{p, i}(z, \mu)=\int_{z_{i-1}<z^{+}<z_{i}} d z^{+} \int \mathrm{d} \mu^{-} \mathrm{K}\left(z, \mu \rightarrow z^{+}, \mu^{-\rho}\right) \tau_{p, i}\left(z^{+}, \mu^{-\prime}\right)+[1-H(\mu)] \\
& \times\left(\frac{1}{m_{i-1}}\right) \mathrm{T}\left(z \rightarrow z_{i-1}, \mu\right) \tau_{p, z_{i-1}}\left(z_{i-1}, \mu\right)+m_{i} \cdot \int_{z_{i}<z^{+}<z_{i+1}} d z^{+} \int d \mu^{\prime} \\
& \times K\left(z, \mu \rightarrow z^{+}, \mu^{\prime \prime}\right) \tau_{p, i+1}\left(z^{+}, \mu^{\prime \prime}\right)+H(\mu) \cdot\left(\mathrm{m}_{i} \mathrm{~m}_{i+1}\right) \mathrm{T}\left(z \rightarrow z_{i+1}, \mu\right) \\
& \times \tau_{p, z_{i+1}}\left(z_{i+1}, \mu\right)+m_{i} \cdot \int d z^{+} \frac{\Sigma_{T}}{|\mu|} T\left(z \rightarrow z^{+}, \mu\right) \cdot \tau_{c} \\
& z_{i}<z^{+}<z_{i+1} \\
& +H(\mu) \cdot m_{i} \cdot T\left(z \rightarrow z_{i+1}, \mu\right) \cdot \tau_{s, i+1}+\int d z^{+} \frac{\bar{L}_{T}}{|\mu|} \\
& z_{i-1}<z^{+}<z_{i} \\
& \times \mathrm{T}\left(z \rightarrow z^{+}, \mu\right) \cdot \tau_{c}+[1-H(\mu)] T\left(z \rightarrow z_{i-1}, \mu\right) \cdot \tau_{R, i-1}+H(\mu) \\
& x \mathrm{~T}\left(z \rightarrow z_{i}, \mu\right) \cdot \tau_{s, i}
\end{aligned}
$$

Note that $H(\mu)$ has been dropped from the third and fifth terms of Eq. (I.4) because the restriction to positive $\mu$ is implicit in the definition of the transition kernel $\mathrm{K}\left(\mathrm{z}, \mu \rightarrow \mathrm{z}^{+}, \mu^{\prime \prime}\right)$ in the third term and implicit in the definition of the transmission probability $\mathrm{T}\left(\mathrm{z} \rightarrow \mathrm{z}^{+}, \mu\right)$ in the fifth term. Recall that $z_{\mathbf{i}-1}<z<\mathbf{z}_{\mathbf{i}}$ in Eq. (1.4). Similar substitution of Eq. (I.2) into the second term of Eq. (I.3) yields [with only terms of Eq. (I.2) that do not vanish for $\mu<0$ contributing]: 


$$
\begin{aligned}
& \tau_{p, i+1}(z, \mu)=\int_{z_{i}<z^{+}<z_{i+1}} \mathrm{~d} z^{+} \int \mathrm{d} \mu^{\prime \prime} \mathrm{k}\left(\mathrm{z}, \mu \rightarrow \mathrm{z}^{+}, \mu^{\prime \prime}\right) \tau_{\mathrm{p}, i+1}\left(z^{+}, \mu^{\prime \prime}\right) \\
& +\left(\frac{1}{m_{i}}\right) \cdot \int_{z_{i-1}^{<z^{+}<z_{i}}} d z^{+} \int \mathrm{d} \mu^{\prime \prime} \mathrm{k}\left(z, \mu \rightarrow z^{+}, \mu^{-\rho}\right) \tau_{p, i}\left(z^{+}, \mu^{-\infty}\right) \\
& +[1-H(\mu)] \cdot\left(\frac{1}{m_{i}{ }^{1} n_{-1}}\right) \cdot T\left(z \rightarrow z_{i-1}, \mu\right) \tau_{p, z_{i-1}}\left(z_{i-1}, \mu\right) \\
& +\left(\frac{1}{m_{i}}\right) \cdot \int_{z_{i-1}<z^{+}<z_{i}} \mathrm{dz}+\frac{\Sigma_{\mathrm{T}}}{|\mu|} \mathrm{T}\left(z \rightarrow z^{+}, \mu\right) \cdot \tau_{c}+[1-\mathrm{H}(\mu)] \cdot\left(\frac{1}{m_{i}}\right) \\
& \times T\left(z \rightarrow z_{i-1}, \mu\right) \cdot \tau_{R, i-1}+H(\mu) \cdot m_{i+1} \cdot T\left(z \rightarrow z_{i+1}, \mu\right) \\
& \times \tau_{p, z_{i+1}}\left(z_{i+1}, \mu\right)+\int_{z_{i}<z^{+}<z_{i+1}} \mathrm{dz}+\frac{\Sigma_{T}}{|\mu|} \mathrm{T}\left(z \rightarrow z^{+}, \mu\right) \cdot \tau_{c} \\
& +[1-H(\mu)] T\left(z \rightarrow z_{i}, \mu\right) \cdot \tau_{R, i}+H(\mu) T\left(z \rightarrow z_{i+1}, \mu\right) \cdot \tau_{s, i+1} \text {. }
\end{aligned}
$$

Once again, we define the following limits:

$$
\begin{aligned}
& \tau_{p}{ }^{-}\left(z_{i}, \mu\right) \equiv \underset{z \rightarrow z_{i-}}{\lim } \tau_{p, i}(z, \mu) \\
& \tau_{p}^{+}\left(z_{i}, \mu\right) \equiv \underset{z \rightarrow z_{1+}}{11 m} \tau_{p, i+1}(z, \mu) .
\end{aligned}
$$


Then, taking the Iimit of Eq. (I.4) as $z$ approaches $z_{i}$ from below and the limit of Eq. (I.5) as $z$ approaches $z_{1}$ from above, we find that for $\mu>0$ :

$$
\tau_{p}^{-}\left(z_{i}, \mu\right)=m_{i} \cdot \tau_{p}^{+}\left(z_{i}, \mu\right)+\tau_{s, i}
$$

Also, for $\mu<0$ :

$$
\tau_{p}^{+}\left(z_{i}, \mu\right)=\frac{1}{m_{i}} \cdot \tau_{p}^{-}\left(z_{i}, \mu\right)+\tau_{R, i} .
$$

Strictly speaking, the condition stated in Eqs. (I.7) applies to a spititing surface with other splituing surfaces on either side. However, the same condition may be derived at $z_{1}$ and $z_{N}$. The discontinuity at surface $i$ presented in Eqs. (I.7a,b) holds for a11 splitting surfaces in the geometry $(i=1,2, \cdots, N)$. Moreover, the discontinuity is a generalization of the single-surface condition derived in Appendix $\mathrm{C}$ [see Eqs. (C.2.6) and (C.2.7)]

The conversion of the formulations for $\tau_{p}(z, \mu)$ to an integrodifferential form proceeds as in Section 4.2.2. In this case, however, there are equations for $N+1$ (rather than two) regions. As an example of the conversion procedure, Eq. (I.1) may be rewritten as follows [using Identities (B.2.2) and (4.1.2.3)]:

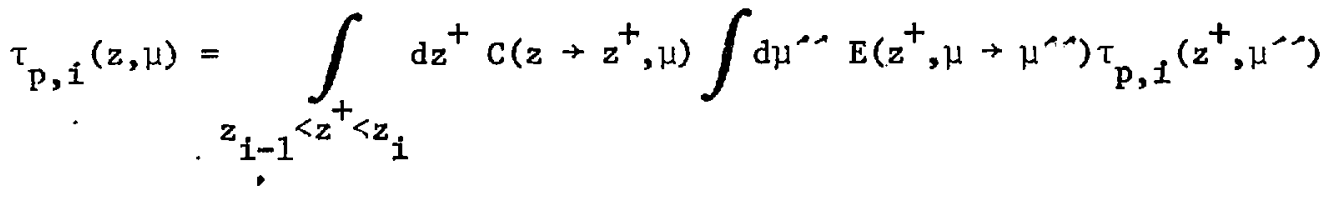

$$
\begin{aligned}
& +\int_{z_{1-1}<z^{+}<z_{1}} d^{+} c\left(z+z^{+}, \mu\right) \cdot \tau_{c}+s_{p, 1}(z, \mu),
\end{aligned}
$$


where

$$
\begin{aligned}
S_{p, i}(z, \mu) & =[1-H(\mu)] \cdot\left(\frac{1}{m_{i-1}}\right) \cdot T\left(z \rightarrow z_{i-1}, \mu\right) \tau_{p, z_{i-1}}\left(z_{i-1}, \mu\right) \\
& +H(\mu) \cdot m_{i} \cdot T\left(z \rightarrow z_{i}, \mu\right) \tau_{p, z_{i}}\left(z_{i}, \mu\right)+[1-H(\mu)] T\left(z \rightarrow z_{i-1}, \mu\right) \\
& \times \tau_{R, i-1}+H(\mu) T\left(z \rightarrow z_{i}, \mu\right) \cdot \tau_{s, i} .
\end{aligned}
$$

Now, by defining

$$
\mathrm{G}^{+}\left(z^{+}, \mu\right) \equiv \int \mathrm{d} \mu^{-} \mathrm{E}\left(z^{+}, \mu+\mu^{-\rho}\right) \tau_{\mathrm{p}, \mathrm{i}}\left(z^{+}, \mu^{-}\right)+\tau_{\mathrm{c}},
$$

Eq. (I.8) may be written as

$$
\tau_{p, i}(z, \mu)=\int_{z_{i-1}^{<z^{+}<z_{i}}} d z^{+} c\left(z \rightarrow z^{+}, \mu\right) G^{+}\left(z^{+}, \mu\right)+s_{p, i}(z, \mu)
$$

By following the same steps as in Section 4.2.2 [see Eqs. (4.2.2.2) through (4.2.2.9)], Eq. (I.II) may be converted to the appropriate integro-differential form:

$$
\begin{aligned}
-\mu \frac{\partial}{\partial z} F_{T, i}(z, \mu)+{ }_{i} T_{T, i}(z, \mu) & =\int d \mu \cdots \Sigma_{T} \cdot E(z, \mu+\mu \cdots) F_{T, i}(z, \mu \cdots) \\
& +\tau_{c}+[1-H(\mu)] \delta\left(z-z_{i-1}\right) \frac{|\mu|}{\Sigma_{T}}\left\{\left(\frac{1}{m_{i-1}}\right) \cdot \tau_{p, z_{i-1}}\left(z_{i-1}, \mu\right)+\tau_{R, i-1}\right\} \\
& +H(\mu) \delta\left(z-z_{i}\right) \frac{\mu}{\Sigma_{T}}\left\{m_{i} \cdot \tau_{p, z_{i}}\left(z_{i}, \mu\right)+\tau_{s, i}\right\},
\end{aligned}
$$

where $\quad \mathrm{F}_{\mathrm{T}, \mathrm{i}}(\mathrm{z}, \mu) \equiv 1 / \Sigma_{\mathrm{T}} \cdot \tau_{\mathrm{p}, \mathrm{i}}(\mathrm{z}, \mu)$ 
In the same manner, but starting with the integral equation governing $\tau_{p, 1}(z, \mu)$ for particles beginning their next flight at $z$ in region 1 , an integro-differential equation may be derived for $\tau_{p, 1}(z, \mu)$. Before the conversion, however, the substitution and integration procedure (discussed at the beginning of this Appendix) should be applied to Eqs. (5.2.8a,b). This will facilitate writing the integral equation for $\tau_{p, 1}(z, \mu)$ in a form similar to that of Eqs. (I.8) and (I.9). From there, the conversion to an integro-differential equation yields for $0 \leq z<z_{1}$ [c.f. Eq. $(4.2 .2 .9)]$ :

$$
\begin{aligned}
& -\mu \frac{\partial}{\partial z} F_{T, 1}(z, \mu)+\Sigma_{T} F_{T, 1}(z, \mu)=\int \mathrm{d} \mu^{\mu} \Sigma_{T} \cdot E\left(z, \mu \rightarrow \mu^{-\prime}\right) F_{T, 1}(z, \mu \cdots) \\
& +\tau_{c}+[1-H(\mu)] \delta(z-0) \frac{|\mu|}{\Sigma_{T}} \cdot \tau_{0}+H(\mu) \delta\left(z-z_{1}\right) \frac{\mu}{\Sigma_{T}} \\
& \quad \times\left\{m_{1} \cdot \tau_{p, z_{1}}\left(z_{1}, \mu\right)+\tau_{s, 1}\right\}
\end{aligned}
$$

where $\quad F_{T, 1}(z, \mu) \equiv 1 / \Sigma_{T} \cdot \tau_{P, 1}(z, \mu) \quad$.

Similarly, starting with Eqs. $(5.2 .12 a, b)$, the following equation may be obtained for starting locations $z$ in the range $z_{N}<z \leq D$ : 


$$
\begin{aligned}
& -\mu \frac{\partial}{\partial z} F_{T, N+1}(z, \mu)+\Sigma_{T^{T}, N+1}(z, \mu)=\int d \mu^{-} \Sigma_{T} \cdot E\left(z, \mu+\mu^{-\rho}\right) F_{T, N+1}\left(z, \mu^{-\mu}\right) \\
& +\tau_{c}+[1-H(\mu)] \delta\left(z-z_{N}\right) \frac{|\mu|}{\Sigma_{T}}\left\{\left(\frac{1}{m_{N}}\right) \cdot \tau_{p, z_{N}}\left(z_{N}, \mu\right)+\tau_{K, N}\right\} \\
& +H(\mu) \delta(z-D) \frac{\mu}{\Sigma_{T}} \cdot \tau_{T} \text {. }
\end{aligned}
$$

Inspection of Eqs. (I.12), (I.13), and (I.14) reveals that the homogeneous form of all equations (regardless of region) is equivalent. In fact, as in Section 4.2, a single equation may be written for $F_{T}(z, \mu)$ $\left[\equiv 1 / \Sigma_{\mathrm{T}} \cdot \tau_{\mathrm{p}}(z, \mu)\right]$ at $a 11 \mathrm{z}$ not on a splitting surface. Thus, for $z \neq z_{\frac{1}{1}}$ $(i=1,2, \cdots, N)$,

$$
\begin{aligned}
& -\mu \frac{\partial}{\partial z} F_{T}(z, \mu)+\Sigma_{T} F_{T}(z, \mu)=\int d \mu \Sigma_{T} \cdot E(z, \mu \rightarrow \mu-\rho) F_{T}(z, \mu-\rho) \\
& +\tau_{c}+[1-H(\mu)] \delta(z-0) \frac{|\mu|}{\Sigma_{T}} \cdot \tau_{0}+H(\mu) \delta(z-D) \frac{\mu}{\Sigma_{T}} \cdot \tau_{T}
\end{aligned}
$$

At each spiitting surface, $z=z_{i}$, the discontinuities characterized by Eqs. (I.7) are imposed on the solution of Eq. (I.15). 


\section{APPENDIX J}

FORTRAN Code Listing:

$\underline{M C S N}$ 
LASL Identification

No, LP-3016

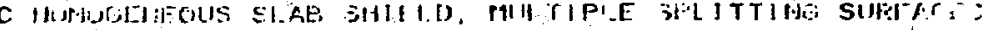

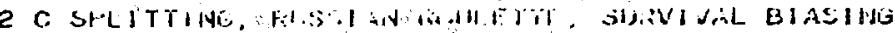

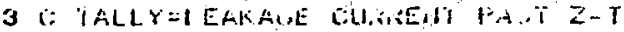

$4 c$

$5 \mathrm{c}$

7

B

9

10

11

12 ci

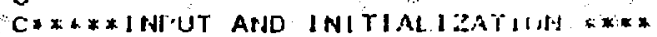

C

REFD(5,5):TITIE

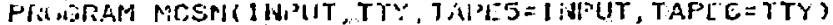

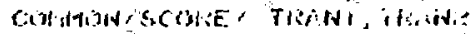

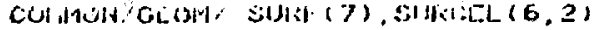

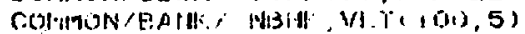

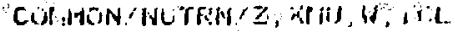

$\Rightarrow \quad F(3.114 T(12 A E)$

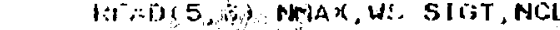

6: HinA ( $12,2: 12,1,12$

REAM

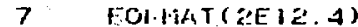

Prins 5,8$)(\operatorname{MP}(1), 1=1, N$ N $)$

FOHAT(G)!2!

$\therefore$

C.

$N:=0$

TLSANI $=$ TRATH $C=0.0$

NLSIUK $=0$

LO $10 \mathrm{~J}=1,100$

Lo $10 \quad J 1=1,5$

$10 \quad$ VI.T(I,J) $=0.0$

$\mathrm{MSl}=\mathrm{NCl}+$ !

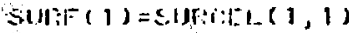

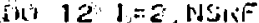

12 Sil $\because(1)=$ SiJfi(:- $-(1-1,2)$

$c$

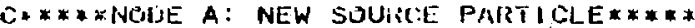

$c$

$20 \quad \mathrm{~N}=\mathrm{N}+1$

$Z=0.0$

$X M U=1.00$

$W=1.00$

$|c| . \mid=1$

SUt T $=0.00$

$c$

C*****NOHE B: RANDOM ISAIKK*****

$c$

C DISTANCE to Cullision

21. R:AIND $=$ RANF $(0.0)$

$D C=(-1 . / S$ I GT $) * A L . C O B$ (RANA )

C DISTANCE TO SUIAFACE INTEBSBFCLION

CAIL DSURF (OS NXSi)

C COLLISION OR SURFACE INTLREECTION 


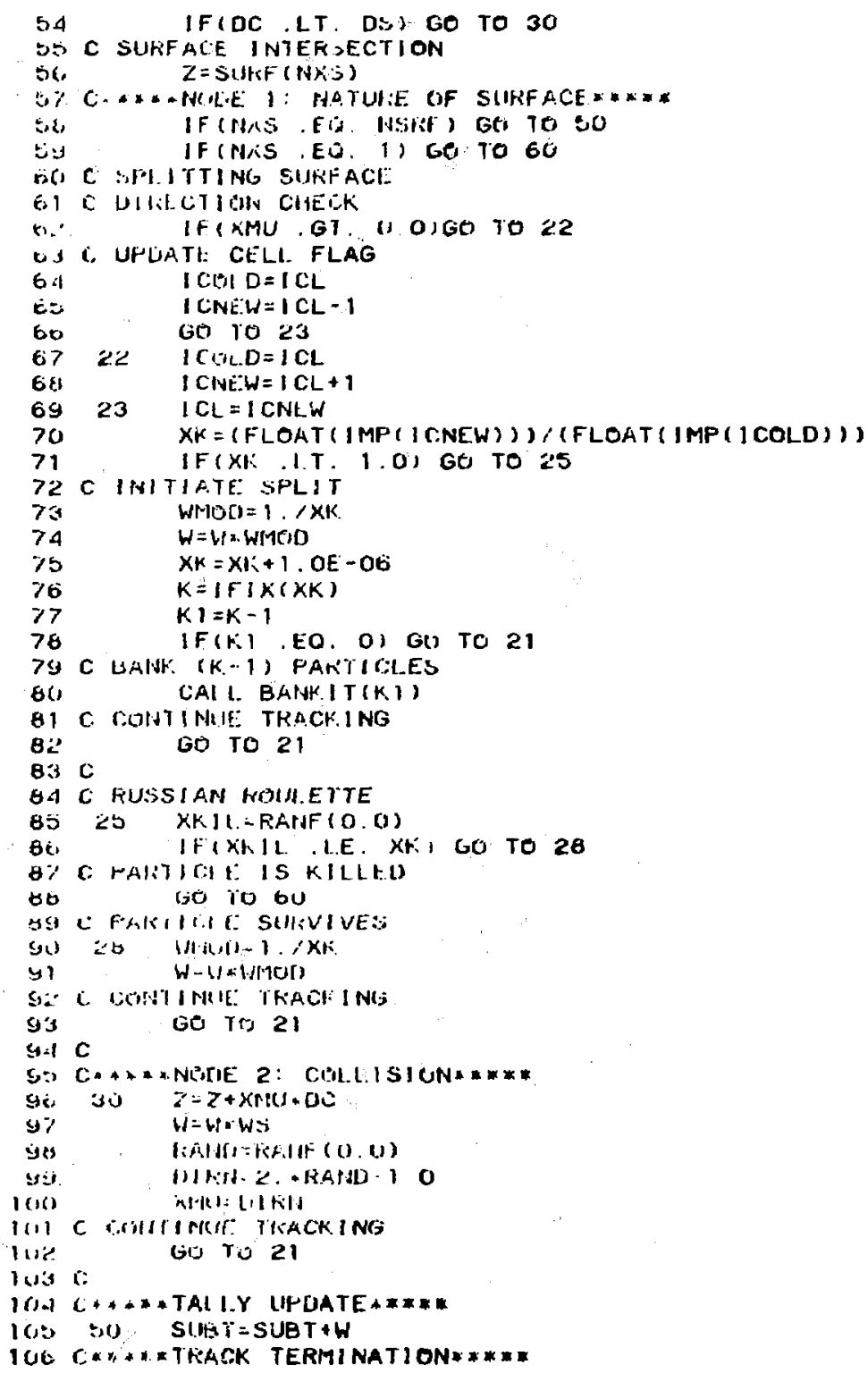



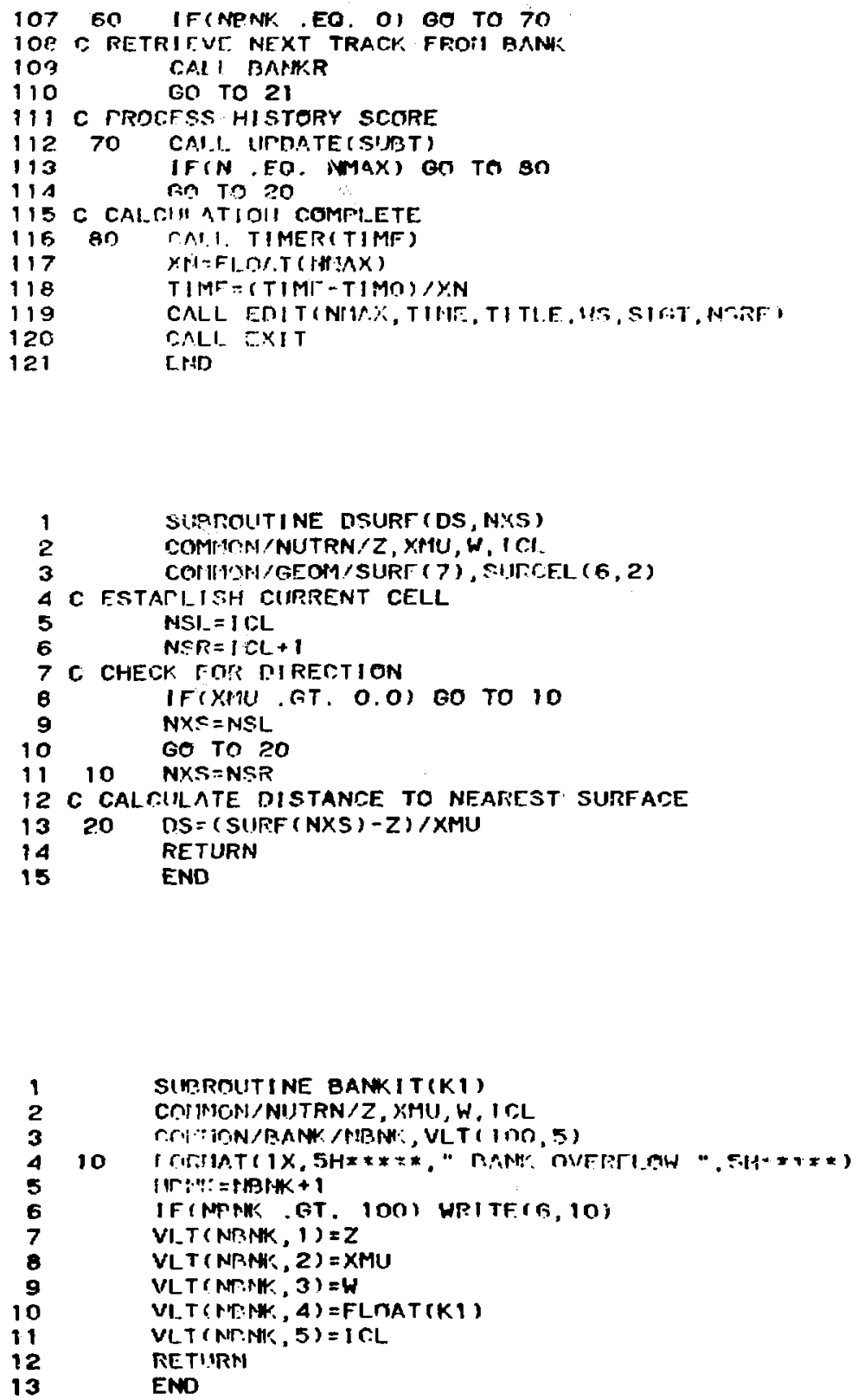
SIEIPUUTIM: EANKF:

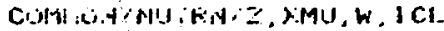

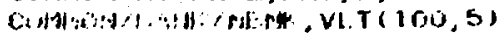

Z-VL T (HISW., 1$)$

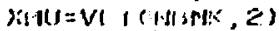

$W=V L T(H I S I N), 3)$

$I C L=V I . T(N I S N:$, 5$)$

$X O L D=V I-T(I K E$ S!E, 4) + I. OE-O6

$K C=1 F I X(X O L D)$

NKiC $=K C-1$

IF (NKC . ED. (I) CO TO 5

XNEH =FLOA I (NICO)

VI.T (THENH: $A)=$ R.NEW

Gi) 1010

Uo $B$ i $C=1,5$

\& VLT $(N I S H K, \mid C)=0.0$

NI:NH: $=$ NLINK -1

IO CUATIINUE

RETURIV

EIND

SI RPROUTINE TIMER(T)

DINIFMSION $12(2)$

$J=200 \mathrm{~B}$

CAL L SYCALL (240AB, J, 12,1)

$T=0.000001 * 12(1)$

F.F TURN

END

\begin{tabular}{|c|c|}
\hline $\mathbf{1}$ & SIMROIITIME UPRATF(SIRET) \\
\hline 2 & CCOHIIIN/SCORE/TRAN1, TSAH? \\
\hline 3 & TRAN 1 - TRAN I + SURT \\
\hline 1 & TRANIZ = TRANZ+SIVET $=2$ \\
\hline 5 & RETUIPN \\
\hline & EWD \\
\hline
\end{tabular}




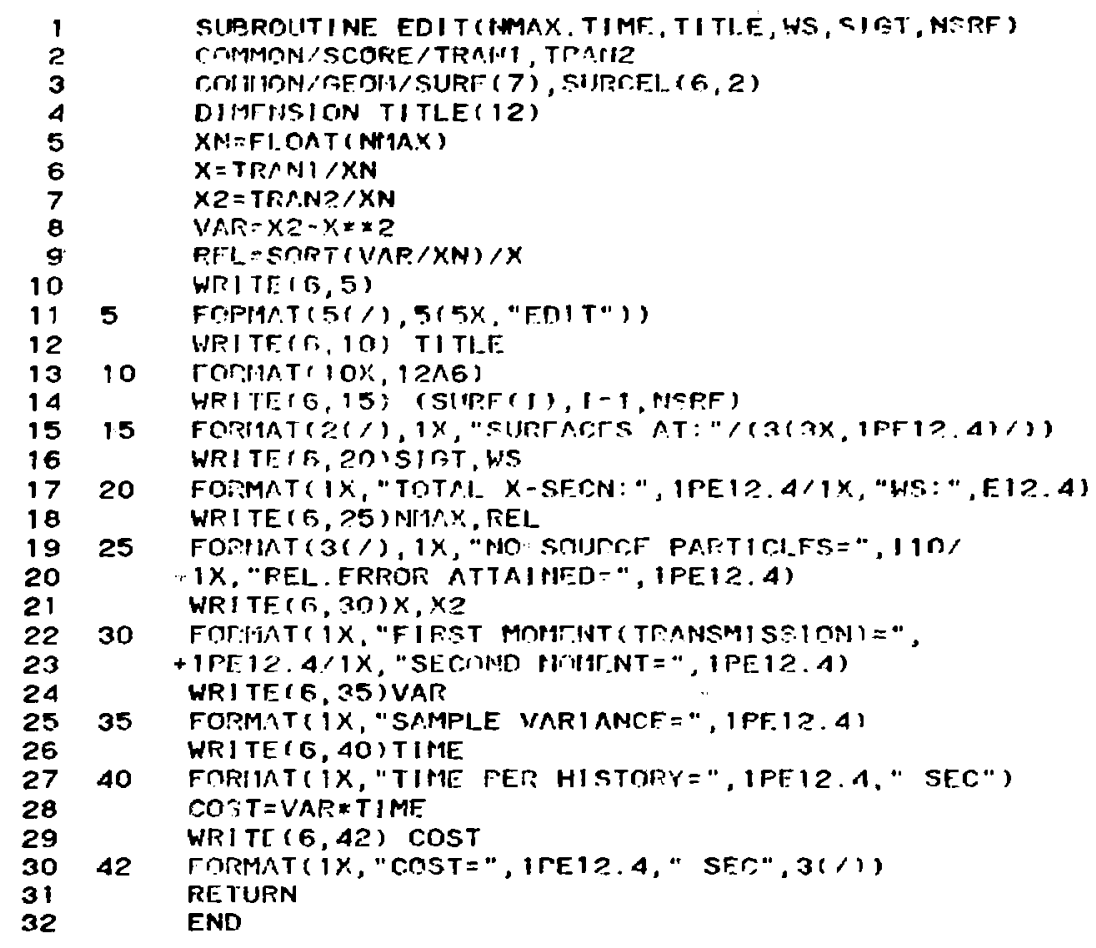

